\title{
Divergent Access to Seven/Five-Membered Rings based on [1,6]-Hydride Shift/Cyclization Process
}

\author{
Daiki Hoshino, $^{\dagger}$ and Keiji Mori ${ }^{\dagger} *$ \\ ${ }^{\dagger}$ Department of Applied Chemistry, Graduate School of Engineering, \\ Tokyo University of Agriculture and Technology, \\ 2-24-16 Nakacho, Koganei, Tokyo \\ 184-8588, Japan. \\ k_mori@cc.tuat.ac.jp
}

Supporting Information

Table of contents

$\mathrm{S} 1$

General experimental procedures $\quad$ S2

$\begin{array}{ll}\text { Procedure and spectral data } & \mathrm{S} 3\end{array}$

Scanned images of ${ }^{1} \mathrm{H}-,{ }^{13} \mathrm{C}-\mathrm{NMR}$, and ${ }^{19} \mathrm{~F}-\mathrm{NMR}$ of new compounds $\quad$ S30 


\section{General experimental procedures}

All reactions utilizing air- and moisture-sensitive reagents were performed in dried glassware under an atmosphere of dry nitrogen. Anhydrous ethereal solvents (THF, $\left.\mathrm{Et}_{2} \mathrm{O}\right)$ were purchased from Kanto Chemical Co., INC., and used directly. Dichloromethane and 1,2-dichloroethane were distilled over $\mathrm{CaH}_{2}$. Benzene and toluene were distilled over $\mathrm{CaH}_{2}$, and stored over 4A molecular sieves. $N, N$-Dimethylformamide (DMF) was distilled over $\mathrm{CaH}_{2}$, and stored over 4A molecular sieves. $\mathrm{M}(\mathrm{OTf})_{\mathrm{n}}$ such as $\mathrm{Sc}(\mathrm{OTf})_{3}, \mathrm{Yb}(\mathrm{OTf})_{3}, \mathrm{Gd}(\mathrm{OTf})_{3}$ and $\mathrm{Mg}(\mathrm{OTf})_{2}$ and $\mathrm{Tf}_{2} \mathrm{NH}$ were used directly in a purchased grade. $\mathrm{BF}_{3} \bullet \mathrm{OEt}_{2}$ and $\mathrm{TiCl}_{4}$ were used after the distillation from $\mathrm{CaH}_{2}$.

For thin-layer chromatography (TLC) analysis, Merck pre-coated plates (silica gel $60 \mathrm{~F}_{254}$, Art 5715, $0.25 \mathrm{~mm}$ ) were used. Column chromatography and preparative TLC (PTLC) were performed on Silica Gel 60N (spherical, neutral), Kanto Chemical Ltd. and Wakogel B-5F, Wako Pure Chemical Industries, respectively.

Melting point (mp) determinations were performed by using a AS ONE ATM-01 instrument and are uncorrected. $\quad{ }^{1} \mathrm{H}$ NMR, ${ }^{13} \mathrm{C}$ NMR were measured on a AL-300 MR (JEOL Ltd., 300 MHz) and ECX-400 (JEOL Ltd., 400 MHz) spectrometers. Chemical shifts are expressed in parts per million (ppm) downfield from internal standard (tetramethylsilane for ${ }^{1} \mathrm{H}$, and $\mathrm{C}_{6} \mathrm{~F}_{6}$ for ${ }^{19} \mathrm{~F}, 0.00 \mathrm{ppm}$ ), and coupling constants are reported as hertz $(\mathrm{Hz})$. Splitting patterns are indicated as follows: br, broad; s, singlet; $\mathrm{d}$, doublet; $\mathrm{t}$, triplet; $\mathrm{m}$, multiplet. Infrared (IR) spectra were recorded on a FTIR-8600PC instrument (Shimadzu Co.). Elemental analysis (EA) was carried out on Flash2000 instrument (Amco Inc.). 


\section{Preparation of starting materials.}

Scheme 1. Preparation of starting materials 3. Preparation of 3a was shown as a representative example.

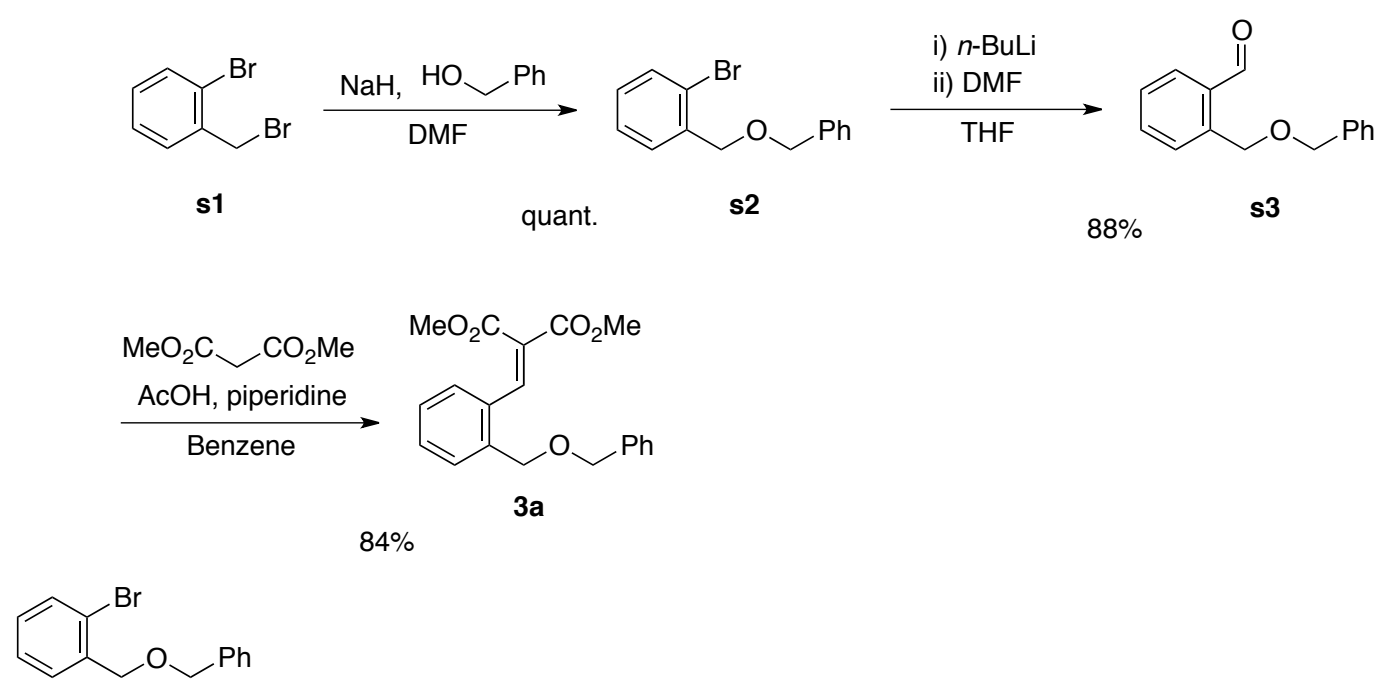

Synthesis of 1-((benzyloxy)methyl)-2-bromobenzene (s2):

To a solution of benzylalcohol $(0.747 \mathrm{~g}, 6.91 \mathrm{mmol})$ in DMF (17.3 mL) were successively added $\mathrm{NaH}$ (60\% oil, $553 \mathrm{mg}, 13.8 \mathrm{mmol}, 2.00$ equiv.), and commercially available s1 (1.73 g, $6.91 \mathrm{mmol}, 1.00$ equiv.). After being stirred for $24 \mathrm{~h}$ at room temperature, the reaction was quenched by addition of $\mathrm{NaHCO}_{3}$ at $0{ }^{\circ} \mathrm{C}$. The crude mixture was extracted with EtOAc (x3) and the combined organic extracts were washed with brine, dried $\left(\mathrm{Na}_{2} \mathrm{SO}_{4}\right)$, and concentrated in vacuo. The residue was purified by to give s2 (1.68 g, $6.91 \mathrm{mmol}$, quant.) as colorless oil.

IR (neat) 3063, 3030, 2923, 2857, 1569, 1496, 1469, 1453, 1440, 1406, 1387, 1356, $1308,1271,1250,1206,1120,1096,1076,1044,1027 \mathrm{~cm}^{-1}$.

${ }^{1} \mathrm{H}$ NMR $\left(300 \mathrm{MHz}, \mathrm{CDCl}_{3}\right) \delta$ 7.59-7.48 (m, 2H), 7.46-7.25 (m, 6H), $7.15(\mathrm{dd}, 1 \mathrm{H}, J=$ 7.5, $7.5 \mathrm{~Hz}), 4.65$ (s, 2H), 4.63 (s, 2H).

${ }^{13} \mathrm{C}$ NMR $\left(75 \mathrm{MHz}, \mathrm{CDCl}_{3}\right) \delta 137.8,137.4,132.2,128.8,128.6,128.1,127.4,127.1$, $122.4,72.4,71.2$.

Anal. Calcd for $\mathrm{C}_{14} \mathrm{H}_{13} \mathrm{BrO}$ : C, 60.67; H, 4.73. Found: C, 60.83; H, 4.46.<smiles>O=Cc1ccccc1COCc1ccccc1</smiles>

Synthesis of 2-((benzyloxy)methyl)benzaldehyde (s3): 
To a solution of $\mathbf{s} 2(1.68 \mathrm{~g}, 6.08 \mathrm{mmol})$ in THF $(20.0 \mathrm{~mL})$ was added $n$-BuLi (1.57 M in hexane, $4.60 \mathrm{~mL}, 7.30 \mathrm{mmol}, 1.20$ equiv.) at $-78{ }^{\circ} \mathrm{C}$. The reaction mixture was stirred for $5 \mathrm{~min}$ at $-78{ }^{\circ} \mathrm{C}$, to which $\mathrm{DMF}(0.94 \mathrm{~mL}, 12.3 \mathrm{mmol}, 2.02$ equiv.) was added. After being stirred for $1.5 \mathrm{~h}$, the reaction was quenched by addition of saturated aqueous $\mathrm{NH}_{4} \mathrm{Cl}$ at $-78{ }^{\circ} \mathrm{C}$. The crude mixture was extracted with EtOAc (x3) and the combined organic extracts were washed with brine, dried $\left(\mathrm{Na}_{2} \mathrm{SO}_{4}\right)$ and concentrated in vacuo. The residue was purified by column chromatography (silica gel, hexane/EtOAc $=8 / 1)$ to afford aldehyde $\mathbf{s 3}(1.21 \mathrm{~g}, 5.35 \mathrm{mmol}, 88 \%)$ as colorless oil.

IR (neat) 3063, 3031, 2858, 2736, 1695, 1601, 1576, 1496, 1454, 1355, 1199, 1160, $1119,1088,1074 \mathrm{~cm}^{-1}$.

${ }^{1} \mathrm{H}$ NMR $\left(300 \mathrm{MHz}, \mathrm{CDCl}_{3}\right) \delta 10.22(\mathrm{~s}, 1 \mathrm{H}), 7.88(\mathrm{dd}, 1 \mathrm{H}, J=7.5,1.5 \mathrm{~Hz}), 7.70(\mathrm{~d}, 1 \mathrm{H}$, $J=7.5 \mathrm{~Hz}$ ), $7.61(\mathrm{ddd}, 1 \mathrm{H}, J=7.5,7.5,1.5 \mathrm{~Hz}), 7.49$ (dd, $1 \mathrm{H}, J=7.5,7.5 \mathrm{~Hz}), 7.45-$ $7.24(\mathrm{~m}, 5 \mathrm{H}), 4.99(\mathrm{~s}, 2 \mathrm{H}), 4.67(\mathrm{~s}, 2 \mathrm{H})$.

${ }^{13} \mathrm{C}$ NMR $\left(75 \mathrm{MHz}, \mathrm{CDCl}_{3}\right) \delta$ 192.8, 140.8, 137.9, 133.8, 133.3, 132.4, 128.4, 128.2, $127.7,127.7,72.8,69.4$.

Anal. Calcd for $\mathrm{C}_{15} \mathrm{H}_{14} \mathrm{O}_{2}$ : C, 79.62; H, 6.24. Found: C, 79.86; H, 6.47.<smiles>COC(=O)C(=Cc1ccccc1COCc1ccccc1)C(C)=O</smiles>

Synthesis of dimethyl 2-(2-((benzyloxy)methyl)benzylidene)malonate (3a) $:^{1}$

To a solution of $\mathbf{s 3}(1.21 \mathrm{~g}, 5.33 \mathrm{mmol})$ in benzene $(17.8 \mathrm{~mL})$ were successively added dimethyl malonate $(0.73 \mathrm{~mL}, 6.40 \mathrm{mmol}, 1.20$ equiv.), piperidine $(0.63 \mathrm{~mL}, 6.40 \mathrm{mmol}$, 1.20 equiv.), and $\mathrm{AcOH}(0.37 \mathrm{~mL}, 6.40 \mathrm{mmol}, 1.20$ equiv.) at room temperature, and the heated to reflux. After being stirred for $17 \mathrm{~h}$ at refluxing temperature. The crude mixture was concentrated in vacuo, and the residue was purified by column chromatography. The residue was purified by column chromatography (silica gel, hexane/EtOAc $=4 / 1)$ to afford $3 \mathbf{a}(1.53 \mathrm{~g}, 4.48 \mathrm{mmol}, 84 \%)$ as colorless oil.

IR (neat) 3063, 3031, 2951, 2856, 1733, 1629, 1454, 1436, 1362, 1262, 1218, 1111, $1069,944 \mathrm{~cm}^{-1}$.

${ }^{1} \mathrm{H}$ NMR $\left(300 \mathrm{MHz}, \mathrm{CDCl}_{3}\right) \delta 8.13(\mathrm{~s}, 1 \mathrm{H}), 7.45-7.24(\mathrm{~m}, 9 \mathrm{H}), 4.61$ (s, 2H), 4.58 (s, 
$2 \mathrm{H}), 3.85(\mathrm{~s}, 3 \mathrm{H}), 3.71(\mathrm{~s}, 3 \mathrm{H})$.

${ }^{13} \mathrm{C}$ NMR $\left(75 \mathrm{MHz}, \mathrm{CDCl}_{3}\right) \delta 166.6,164.1,141.9,137.7,137.1,132.6,129.9,129.1$, $128.3,128.0,127.7,127.6,127.3,72.4,70.0,52.5,52.4$.

Anal. Calcd for $\mathrm{C}_{20} \mathrm{H}_{20} \mathrm{O}_{5}$ : C, 70.57; H, 5.92. Found: C, 70.36; H, 6.11.<smiles>Cc1ccc(COCc2ccccc2)c(Br)c1</smiles>

1-((Benzyloxy)methyl)-2-bromo-4-methylbenzene (s4).

Colorless oil (purified by silica gel column chromatography, Hexane/EtOAc $=20 / 1$ ).

Yield: 413 mg (84\%, synthesized from 2-bromo-1-(bromomethyl)-4-methylbenzene ${ }^{1}$ ).

IR (neat) 3063, 3030, 2920, 2857, 1607, 1559, 1541, 1521, 1507, 1495, 1472, 1454, $1418,1247,1210,1143,1098,1074,1040,1029,964 \mathrm{~cm}^{-1}$.

${ }^{1} \mathrm{H}$ NMR (300 MHz, $\left.\mathrm{CDCl}_{3}\right) \delta$ 7.45-7.24 (m, 7H), $7.12(\mathrm{~d}, 1 \mathrm{H}, J=8.1 \mathrm{~Hz}), 4.62(\mathrm{~s}, 2 \mathrm{H})$, $4.60(\mathrm{~s}, 2 \mathrm{H}), 2.32(\mathrm{~s}, 3 \mathrm{H})$.

${ }^{13} \mathrm{C}$ NMR $\left(75 \mathrm{MHz}, \mathrm{CDCl}_{3}\right) \delta 139.0,138.1,134.4,132.9,129.1,128.3,128.1,127.7$, 127.6, 122.7, 72.5, 71.3, 20.7 .

Anal. Calcd for $\mathrm{C}_{15} \mathrm{H}_{15} \mathrm{BrO}$ : C, 61.87; H, 5.19. Found: C, 61.95; H, 5.03.<smiles>Cc1ccc(COCc2ccccc2)c(C=O)c1</smiles>

2-((Benzyloxy)methyl)-5-methylbenzaldehyde (s5).

Colorless oil (purified by silica gel column chromatography, Hexane/EtOAc $=8 / 1$ ).

Yield: $286 \mathrm{mg}$ (76\%, synthesized from $\mathbf{s 4}$ ).

IR (neat) 30303062, 3030, 2922, 2859, 2733, 1689, 1609, 1571, 1497, 1454, 1400, 1386, 1356, 1302, 1282, 1241, 1205, 1156, 1126, 1091, 1073, 1028, $964 \mathrm{~cm}^{-1}$.

${ }^{1} \mathrm{H}$ NMR (300 MHz, $\left.\mathrm{CDCl}_{3}\right) \delta 10.21(\mathrm{~s}, 1 \mathrm{H}), 7.68(\mathrm{~s}, 1 \mathrm{H}), 7.54(\mathrm{~d}, 1 \mathrm{H}, J=7.5 \mathrm{~Hz})$, 7.44-7.23 (m, 6H), 4.93 (s, 2H), 4.64 (s, 2H), 2.42 (s, 3H).

${ }^{13} \mathrm{C}$ NMR $\left(75 \mathrm{MHz}, \mathrm{CDCl}_{3}\right) \delta 192.7,137.9,137.6,137.5,134.4,133.2,132.4,128.4$, $128.3,127.6,127.5,72.5,69.1,20.7$.

Anal. Calcd for $\mathrm{C}_{16} \mathrm{H}_{16} \mathrm{O}_{2}$ : C, 79.97; H, 6.71. Found: C, 80.12; H, 6.97. 


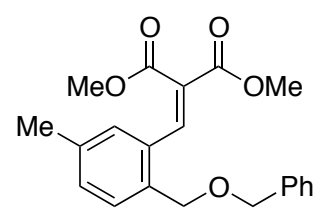

Dimethyl 2-(2-((benzyloxy)methyl)-5-methylbenzylidene)malonate (3b).

Colorless oil (purified by silica gel column chromatography, Hexane/EtOAc $=4 / 1$ ).

Yield: $316 \mathrm{mg}$ (78\%, synthesized from s5).

IR (neat) 3030, 2951, 2858, 1733, 1629, 1496, 1454, 1436, 1363, 1268, 1228, 1162, $1116,1071,1028,987,925 \mathrm{~cm}^{-1}$.

${ }^{1} \mathrm{H}$ NMR (300 MHz, $\left.\mathrm{CDCl}_{3}\right) \delta 8.12(\mathrm{~s}, 1 \mathrm{H}), 7.47-7.26(\mathrm{~m}, 6 \mathrm{H}), 7.24-7.12(\mathrm{~m}, 2 \mathrm{H}), 4.57$ (s, 2H), 4.55 (s, 2H), 3.85 (s, 3H), $3.72(\mathrm{~s}, 3 \mathrm{H}), 2.32$ (s, 3H).

${ }^{13} \mathrm{C}$ NMR $\left(75 \mathrm{MHz}, \mathrm{CDCl}_{3}\right) \delta 166.5,164.0,141.8,137.7,137.6,134.2,132.4,130.5$, $129.2,128.2,128.1,127.6,127.4,126.8,72.0,69.7,52.3,52.1,20.8$.

Anal. Calcd for $\mathrm{C}_{21} \mathrm{H}_{22} \mathrm{O}_{5}$ : C, 71.17; H, 6.26. Found: C, 71.02; H, 6.13.<smiles>COc1ccc(COCc2ccccc2)c(Br)c1</smiles>

1-((Benzyloxy)methyl)-2-bromo-4-methoxybenzene (s6).

Colorless oil (purified by silica gel column chromatography, Hexane/EtOAc $=20 / 1$ ).

Yield: $384 \mathrm{mg}$ (91\%, synthesized from 2-bromo-1-(bromomethyl)-4-methoxybenzene ${ }^{2}$ ). IR (neat) 3063, 3030, 3005, 2940, 2904, 2856, 2837, 1605, 1569, 1495, 1455, 1439, $1397,1387,1357,1311,1284,1240,1182,1094,1072,1035,936 \mathrm{~cm}^{-1}$.

${ }^{1} \mathrm{H}$ NMR (300 MHz, $\left.\mathrm{CDCl}_{3}\right) \delta$ 7.45-7.24 (m, 6H), $7.11(\mathrm{~d}, 1 \mathrm{H}, J=2.4 \mathrm{~Hz}), 6.86(\mathrm{dd}$, $1 \mathrm{H}, J=8.4,2.4 \mathrm{~Hz}), 4.60$ (s, 2H), 4.57 (s, 2H), 3.80 (s, 3H).

${ }^{13} \mathrm{C}$ NMR $\left(75 \mathrm{MHz}, \mathrm{CDCl}_{3}\right) \delta 159.4,138.1,130.3,129.4,128.3,127.7,127.6,123.6$, $117.8,113.2,72.3,71.1,55.4$.

Anal. Calcd for $\mathrm{C}_{15} \mathrm{H}_{15} \mathrm{BrO}_{2}$ : C, 58.65; H, 4.92. Found: C, 58.50; H, 5.16.<smiles>COc1ccc(COCc2ccccc2)c(C=O)c1</smiles>

2-((Benzyloxy)methyl)-5-methoxybenzaldehyde (s7).

Colorless oil (purified by silica gel column chromatography, Hexane/EtOAc $=8 / 1$ ).

Yield: $214 \mathrm{mg}$ (68\%, synthesized from s6).

IR (neat) 3063, 3031, 3006, 2933, 2905, 2859, 2735, 1692, 1606, 1575, 1500, 1455, 
1426, 1401, 1389, 1358, 1318, 1281, 1258, 1163, 1112, 1095, 1071, 1036, $962 \mathrm{~cm}^{-1}$. ${ }^{1} \mathrm{H}$ NMR $\left(300 \mathrm{MHz}, \mathrm{CDCl}_{3}\right) \delta 10.26(\mathrm{~s}, 1 \mathrm{H}), 7.48(\mathrm{~d}, 1 \mathrm{H}, J=8.4 \mathrm{~Hz}), 7.42(\mathrm{~d}, 1 \mathrm{H}, J=$ $2.7 \mathrm{~Hz}), 7.39-7.25(\mathrm{~m}, 5 \mathrm{H}), 7.12(\mathrm{dd}, 1 \mathrm{H}, J=2.7,8.4 \mathrm{~Hz}), 4.88(\mathrm{~s}, 2 \mathrm{H}), 4.62(\mathrm{~s}, 2 \mathrm{H})$, $3.87(\mathrm{~s}, 3 \mathrm{H})$.

${ }^{13} \mathrm{C} \mathrm{NMR}\left(75 \mathrm{MHz}, \mathrm{CDCl}_{3}\right) \delta 191.9,159.4,137.8,134.8,132.7,130.8,128.4,127.8$, $127.7,120.1,114.4,72.5,68.8,55.5$.

Anal. Calcd for $\mathrm{C}_{16} \mathrm{H}_{16} \mathrm{O}_{3}$ : C, 74.98; H, 6.29. Found: C, 74.74; H, 6.44.<smiles>COC(=O)C(=Cc1cc(OC)ccc1COCc1ccccc1)C(=O)OC</smiles>

Dimethyl 2-(2-((benzyloxy)methyl)-5-methoxybenzylidene)malonate (3c).

Colorless oil (purified by silica gel column chromatography, Hexane/EtOAc $=4 / 1$ ).

Yield: $255 \mathrm{mg}$ (87\%, synthesized from s7).

IR (neat) 3030, 3004, 2952, 2856, 1732, 1628, 1606, 1574, 1496, 1454, 1436, 1363, $1272,1234,1167,1070,1038,989,926 \mathrm{~cm}^{-1}$.

${ }^{1} \mathrm{H}$ NMR (300 MHz, $\left.\mathrm{CDCl}_{3}\right) \delta 8.11(\mathrm{~s}, 1 \mathrm{H}), 7.47-7.22(\mathrm{~m}, 6 \mathrm{H}), 6.93$ (s, 1H), 6.90 (d, $1 \mathrm{H}, J=8.1 \mathrm{~Hz}), 4.54(\mathrm{~s}, 2 \mathrm{H}), 4.53(\mathrm{~s}, 2 \mathrm{H}), 3.85(\mathrm{~s}, 3 \mathrm{H}), 3.78(\mathrm{~s}, 3 \mathrm{H}), 3.74(\mathrm{~s}, 3 \mathrm{H})$.

${ }^{13} \mathrm{C}$ NMR $\left(75 \mathrm{MHz}, \mathrm{CDCl}_{3}\right) \delta 166.6,164.1,159.2,141.6,137.9,133.9,130.9,129.4$, $128.3,127.8,127.6,127.4,115.5,113.0,72.1,69.6,55.2,52.6,52.5$.

Anal. Calcd for $\mathrm{C}_{21} \mathrm{H}_{22} \mathrm{O}_{6}$ : C, 68.10; H, 5.99. Found: C, 68.18; H, 5.80.<smiles>Fc1ccc(COCc2ccccc2)c(Br)c1</smiles>

1-((Benzyloxy)methyl)-2-bromo-4-fluorobenzene (s8).

Colorless oil (purified by silica gel column chromatography, Hexane/EtOAc $=20 / 1$ ).

Yield: $390 \mathrm{mg}$ (70\%, synthesized from 2-bromo-1-(bromomethyl)-4-fluorobenzene ${ }^{3}$ ).

IR (neat) 3087, 3065, 3031, 2858, 1601, 1589, 1488, 1453, 1386, 1356, 1308, 1262 , $1230,1181,1099,1075,1030,965 \mathrm{~cm}^{-1}$.

${ }^{1} \mathrm{H}$ NMR $\left(300 \mathrm{MHz}, \mathrm{CDCl}_{3}\right) \delta 7.49(\mathrm{dd}, 1 \mathrm{H}, J=8.1,6.0 \mathrm{~Hz}), 7.44-7.25(\mathrm{~m}, 6 \mathrm{H}), 7.04$ (ddd, 1H, $J=8.1,8.1,2.4 \mathrm{~Hz}), 4.63(\mathrm{~s}, 2 \mathrm{H}), 4.58$ (s, 2H).

${ }^{13} \mathrm{C}$ NMR $\left(75 \mathrm{MHz}, \mathrm{CDCl}_{3}\right) \delta 161.7\left(\mathrm{~d}, J_{C-F}=248.5 \mathrm{~Hz}\right), 137.8,133.5\left(\mathrm{~d}, J_{C-F}=3.1 \mathrm{~Hz}\right)$, $130.2\left(\mathrm{~d}, J_{C-F}=8.6 \mathrm{~Hz}\right), 128.4,127.7\left(\mathrm{~d}, J_{C-F}=2.5 \mathrm{~Hz}\right), 122.8,122.7,119.7\left(\mathrm{~d}, J_{C-F}=\right.$ 
$24.0 \mathrm{~Hz}), 114.4\left(\mathrm{~d}, J_{C-F}=20.3 \mathrm{~Hz}\right), 72.8,70.9$.

${ }^{19} \mathrm{~F} \mathrm{NMR}\left(283 \mathrm{MHz}, \mathrm{CDCl}_{3}\right) \delta 48.6(\mathrm{dd}, 1 \mathrm{~F}, J=7.1,14.7 \mathrm{~Hz})$.

Anal. Calcd for $\mathrm{C}_{14} \mathrm{H}_{12} \mathrm{BrFO}$ : C, 56.97; H, 4.10. Found: C, 57.24; H, 4.02.<smiles>O=Cc1cc(F)ccc1COCc1ccccc1</smiles>

2-((Benzyloxy)methyl)-5-fluorobenzaldehyde (s9).

Colorless oil (purified by silica gel column chromatography, Hexane/EtOAc $=8 / 1$ ).

Yield: $257 \mathrm{mg}$ (82\%, synthesized from s8).

IR (neat) 3087, 3065, 3032, 2861, 1696, 1609, 1586, 1496, 1455, 1425, 1402, 1388, $1356,1305,1246,1149,1109,1092,1073,1028,951 \mathrm{~cm}^{-1}$.

${ }^{1} \mathrm{H}$ NMR $\left(300 \mathrm{MHz}, \mathrm{CDCl}_{3}\right) \delta 10.20(\mathrm{~d}, 1 \mathrm{H}, J=1.5 \mathrm{~Hz}), 7.68-7.52(\mathrm{~m}, 2 \mathrm{H}), 7.42-7.24$ $(\mathrm{m}, 6 \mathrm{H}), 4.90(\mathrm{~s}, 2 \mathrm{H}), 4.64(\mathrm{~s}, 2 \mathrm{H})$.

${ }^{13} \mathrm{C}$ NMR $\left(75 \mathrm{MHz}, \mathrm{CDCl}_{3}\right) \delta 190.9\left(\mathrm{~d}, J_{C-F}=1.9 \mathrm{~Hz}\right), 162.1\left(\mathrm{~d}, J_{C-F}=247.2 \mathrm{~Hz}\right), 137.5$, $136.4\left(\mathrm{~d}, J_{C-F}=3.7 \mathrm{~Hz}\right), 135.2\left(\mathrm{~d}, J_{C-F}=5.5 \mathrm{~Hz}\right), 130.8\left(\mathrm{~d}, J_{C-F}=7.4 \mathrm{~Hz}\right), 128.4,127.8$, $127.7,120.6\left(\mathrm{~d}, J_{C-F}=20.9 \mathrm{~Hz}\right), 117.3\left(\mathrm{~d}, J_{C-F}=22.2 \mathrm{~Hz}\right), 72.7,68.6$.

${ }^{19} \mathrm{~F}$ NMR $\left(283 \mathrm{MHz}, \mathrm{CDCl}_{3}\right) \delta 48.4(\mathrm{dd}, 1 \mathrm{~F}, J=7.1,13.9 \mathrm{~Hz})$.

Anal. Calcd for $\mathrm{C}_{15} \mathrm{H}_{13} \mathrm{FO}_{2}$ : C, 73.76; H, 5.36. Found: C, 73.92; H, 5.21.

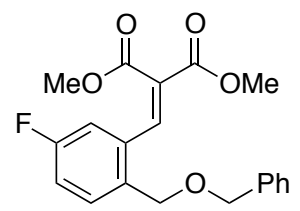

Dimethyl 2-(2-((benzyloxy)methyl)-5-fluorobenzylidene)malonate (3d).

Colorless oil (purified by silica gel column chromatography, Hexane/EtOAc $=4 / 1$ ).

Yield: $260 \mathrm{mg}$ (76\%, synthesized from s9).

IR (neat) 3064, 2032, 3005, 2952, 2859, 1735, 1632, 1610, 1585, 1491, 1454, 1437, $1363,1271,1229,1191,1161,1101,1071,1028,997,934 \mathrm{~cm}^{-1}$.

${ }^{1} \mathrm{H}$ NMR (300 MHz, $\left.\mathrm{CDCl}_{3}\right) \delta 8.04(\mathrm{~s}, 1 \mathrm{H}), 7.45-7.26(\mathrm{~m}, 6 \mathrm{H}), 7.10-7.02(\mathrm{~m}, 2 \mathrm{H}), 4.57$ $(\mathrm{s}, 2 \mathrm{H}), 4.55(\mathrm{~s}, 2 \mathrm{H}), 3.86(\mathrm{~s}, 3 \mathrm{H}), 3.75(\mathrm{~s}, 3 \mathrm{H})$.

${ }^{13} \mathrm{C}$ NMR $\left(75 \mathrm{MHz}, \mathrm{CDCl}_{3}\right) \delta 166.0,163.8,162.0\left(\mathrm{~d}, J_{C-F}=245.4 \mathrm{~Hz}\right), 140.2\left(\mathrm{~d}, J_{C-F}=\right.$ $1.9 \mathrm{~Hz}), 137.6,134.5\left(\mathrm{~d}, J_{C-F}=8.0 \mathrm{~Hz}\right), 133.1\left(\mathrm{~d}, J_{C-F}=3.7 \mathrm{~Hz}\right), 131.0\left(\mathrm{~d}, J_{C-F}=8.6 \mathrm{~Hz}\right)$, $128.4,128.4,127.7,127.7,116.5\left(\mathrm{~d}, J_{C-F}=21.0 \mathrm{~Hz}\right), 114.7\left(\mathrm{~d}, J_{C-F}=23.5 \mathrm{~Hz}\right), 72.4$, $69.3,52.6,52.5$. 
${ }^{19} \mathrm{~F} \mathrm{NMR}\left(283 \mathrm{MHz}, \mathrm{CDCl}_{3}\right) \delta 48.4(\mathrm{dd}, J=9.3,16.1 \mathrm{~Hz})$.

Anal. Calcd for $\mathrm{C}_{20} \mathrm{H}_{19} \mathrm{FO}_{5}$ : C, 67.03; H, 5.34. Found: C, 66.76; H, 5.39.<smiles>Cc1ccc(Br)c(COCc2ccccc2)c1</smiles>

2-((Benzyloxy)methyl)-1-bromo-4-methylbenzene (s10).

Colorless oil (purified by silica gel column chromatography, Hexane/EtOAc $=20 / 1$ ).

Yield: $341 \mathrm{mg}$ (63\%, synthesized from 1-bromo-2-(bromomethyl)-4-methylbenzene ${ }^{4}$ ).

IR (neat) 3078, 3062, 3030, 2921, 2858, 1496, 1469, 1454, 1405, 1386, 1356, 1309, $1277,1241,1205,1160,1142,1098,1027,950 \mathrm{~cm}^{-1}$.

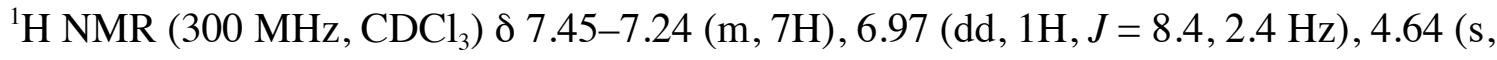
$2 \mathrm{H}), 4.59(\mathrm{~s}, 2 \mathrm{H}), 2.32(\mathrm{~s}, 3 \mathrm{H})$.

${ }^{13} \mathrm{C}$ NMR $\left(75 \mathrm{MHz}, \mathrm{CDCl}_{3}\right) \delta 138.1,137.3,137.1,132.2,129.9,129.7,128.4,127.8$, 127.7, 119.4, 72.7, 71.6, 21.0.

Anal. Calcd for $\mathrm{C}_{15} \mathrm{H}_{15} \mathrm{BrO}$ : C, 61.87; H, 5.19. Found: C, 61.68; H, 5.42.<smiles>Cc1ccc(C=O)c(COCc2ccccc2)c1</smiles>

2-((Benzyloxy)methyl)-4-methylbenzaldehyde (s11).

Colorless oil (purified by silica gel column chromatography, Hexane/EtOAc $=8 / 1$ ).

Yield: $188 \mathrm{mg}$ (73\%, synthesized from s10).

IR (neat) 3062, 3030, 2921, 2857, 2735, 1692, 1607, 1572, 1496, 1454, 1397, 1354, $1305,1286,1244,1205,1158,1125,1188,1074,1028,949 \mathrm{~cm}^{-1}$.

${ }^{1} \mathrm{H}$ NMR $\left(300 \mathrm{MHz}, \mathrm{CDCl}_{3}\right) \delta 10.14(\mathrm{~s}, 1 \mathrm{H}), 7.76(\mathrm{~d}, 1 \mathrm{H}, J=7.8 \mathrm{~Hz}), 7.50(\mathrm{~s}, 1 \mathrm{H})$, 7.44-7.23 (m, 6H), 4.96 (s, 2H), 4.67 (s, 2H), 2.44 (s, 3H).

${ }^{13} \mathrm{C}$ NMR $\left(75 \mathrm{MHz}, \mathrm{CDCl}_{3}\right.$ ) $\delta 192.3,144.9,140.7,137.9,132.8,131.0,128.8,128.3$, 128.2, 127.7, 127.6, 72.8, 69.4, 21.8.

Anal. Calcd for $\mathrm{C}_{16} \mathrm{H}_{16} \mathrm{O}_{2}$ : C, 79.97; H, 6.71. Found: C, 80.24; H, 6.87.

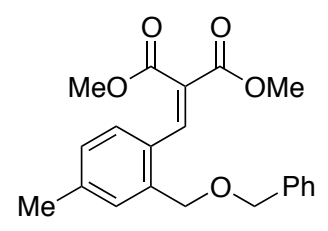


Dimethyl 2-(2-((benzyloxy)methyl)-4-methylbenzylidene)malonate (3e).

Colorless oil (purified by silica gel column chromatography, Hexane/EtOAc $=4 / 1$ ).

Yield: $157 \mathrm{mg}$ (58\%, synthesized from s11).

IR (neat) 3062, 3030, 3004, 2952, 2922, 2857, 1734, 1628, 1611, 1497, 1454, 1436, $1362,1299,1266,1220,1182,1119,1070,1028,986,945 \mathrm{~cm}^{-1}$.

${ }^{1} \mathrm{H}$ NMR $\left(300 \mathrm{MHz}, \mathrm{CDCl}_{3}\right) \delta 8.09(\mathrm{~s}, 1 \mathrm{H}), 7.40-7.22(\mathrm{~m}, 7 \mathrm{H}), 7.09(\mathrm{~d}, 1 \mathrm{H}, J=7.5 \mathrm{~Hz})$, $4.56(\mathrm{~s}, 4 \mathrm{H}), 3.84(\mathrm{~s}, 3 \mathrm{H}), 3.73(\mathrm{~s}, 3 \mathrm{H}), 2.36(\mathrm{~s}, 3 \mathrm{H})$.

${ }^{13} \mathrm{C}$ NMR $\left(75 \mathrm{MHz}, \mathrm{CDCl}_{3}\right) \delta 166.7,164.2,141.5,140.3,137.7,137.1,129.9,129.5$, $128.6,128.2,127.7,127.6,127.5,126.2,72.3,70.0,52.3,52.2,21.1$.

Anal. Calcd for $\mathrm{C}_{21} \mathrm{H}_{22} \mathrm{O}_{5}$ : C, 71.17; H, 6.26. Found: C, 71.32; H, 6.04.

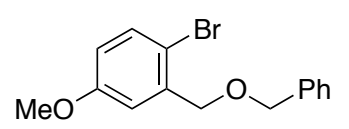

2-((Benzyloxy)methyl)-1-bromo-4-methoxybenzene (s12).

Colorless oil (purified by silica gel column chromatography, Hexane/EtOAc $=20 / 1$ ).

Yield: $\quad 504 \mathrm{mg} \quad(78 \%$, synthesized from commercially available, 1-bromo-2-(bromomethyl)-4-methoxybenzene).

IR (neat) 3063, 3030, 3004, 2945, 2905, 2857, 2837, 1594, 1575, 1473, 1457, 1438, 1419, 1405, 1387, 1356, 1297, 1273, 1238, 1192, 1164, 1096, 1056, 1016, $945 \mathrm{~cm}^{-1}$.

${ }^{1} \mathrm{H}$ NMR (300 MHz, $\left.\mathrm{CDCl}_{3}\right) \delta 7.48-7.24(\mathrm{~m}, 6 \mathrm{H}), 7.11(\mathrm{~d}, 1 \mathrm{H}, J=3.0 \mathrm{~Hz}), 6.71$ (dd, $1 \mathrm{H}, J=8.4,3.0 \mathrm{~Hz}), 4.65(\mathrm{~s}, 2 \mathrm{H}), 4.59(\mathrm{~s}, 2 \mathrm{H}), 3.80(\mathrm{~s}, 3 \mathrm{H})$.

${ }^{13} \mathrm{C}$ NMR $\left(75 \mathrm{MHz}, \mathrm{CDCl}_{3}\right) \delta 158.9,138.5,137.9,132.9,128.3,127.6,114.4,114.2$, 112.6, 72.6, 71.3, 55.3.

Anal. Calcd for $\mathrm{C}_{15} \mathrm{H}_{15} \mathrm{BrO}_{2}$ : C, 58.65; H, 4.92. Found: C, 58.79; H, 4.74.<smiles>COc1ccc(C=O)c(COCc2ccccc2)c1</smiles>

2-((Benzyloxy)methyl)-4-methoxybenzaldehyde (s13).

Colorless oil (purified by silica gel column chromatography, Hexane/EtOAc $=8 / 1$ ).

Yield: $236 \mathrm{mg}$ (58\%, synthesized from s12).

IR (neat) 3063, 3030, 2937, 2842, 2738, 1686, 1602, 1568, 1497, 1454, 1432, 1354, $1319,1286,1259,1243,1210,1165,1125,1091,1075,1029,952 \mathrm{~cm}^{-1}$.

${ }^{1} \mathrm{H}$ NMR $\left(300 \mathrm{MHz}, \mathrm{CDCl}_{3}\right) \delta 10.02(\mathrm{~s}, 1 \mathrm{H}), 7.80(\mathrm{~d}, 1 \mathrm{H}, J=8.4 \mathrm{~Hz}), 7.47-7.25(\mathrm{~m}$, 
$6 \mathrm{H}), 6.93(\mathrm{dd}, 1 \mathrm{H}, J=8.4,2.7 \mathrm{~Hz}), 5.00(\mathrm{~s}, 2 \mathrm{H}), 4.68(\mathrm{~s}, 2 \mathrm{H}), 3.90(\mathrm{~s}, 3 \mathrm{H})$.

${ }^{13} \mathrm{C}$ NMR $\left(75 \mathrm{MHz}, \mathrm{CDCl}_{3}\right) \delta 191.2,163.9,143.6,137.9,135.7,127.6,128.3,127.5$, 126.4, 113.1, 112.1, 72.7, 69.4, 55.3.

Anal. Calcd for $\mathrm{C}_{16} \mathrm{H}_{16} \mathrm{O}_{3}$ : C, 74.98; H, 6.29. Found: C, 75.15; H, 6.03.<smiles>COC(=O)C(=Cc1ccc(OC)cc1COCc1ccccc1)C(=O)OC</smiles>

Dimethyl 2-(2-((benzyloxy)methyl)-4-methoxybenzylidene)malonate (3f).

Colorless oil (purified by silica gel column chromatography, Hexane/EtOAc $=4 / 1$ ).

Yield: $253 \mathrm{mg}$ (75\%, synthesized from $\mathbf{s 1 3})$.

IR (neat) 3062, 3030, 3004, 2952, 2843, 1733, 1603, 1571, 1497, 1454, 1436, 1363, $1310,1258,1220,1182,1115,1104,1070,1045,1029,987,944 \mathrm{~cm}^{-1}$.

${ }^{1} \mathrm{H}$ NMR (300 MHz, $\left.\mathrm{CDCl}_{3}\right) \delta 8.04(\mathrm{~s}, 1 \mathrm{H}), 7.86-7.21(\mathrm{~m}, 6 \mathrm{H}), 7.00(\mathrm{~d}, 1 \mathrm{H}, J=2.7 \mathrm{~Hz})$, $6.80(\mathrm{dd}, 1 \mathrm{H}, J=2.7,8.7 \mathrm{~Hz}), 4.60(\mathrm{~s}, 2 \mathrm{H}), 4.60$ (s, 2H), 3.84 (s, 3H), 3.83 (s, 3H), 3.76 (s, 3H).

${ }^{13} \mathrm{C}$ NMR $\left(75 \mathrm{MHz}, \mathrm{CDCl}_{3}\right) \delta 167.0,164.3,161.0,140.7,139.6,137.6,129.4,128.2$, $127.6,127.5,124.8,124.3,114.5,113.0,72.3,69.7,55.1,52.3,52.3$.

Anal. Calcd for $\mathrm{C}_{21} \mathrm{H}_{22} \mathrm{O}_{6}$ : C, 68.10; H, 5.99. Found: C, 68.27; H, 6.15.<smiles>Fc1ccc(Br)c(COCc2ccccc2)c1</smiles>

2-((Benzyloxy)methyl)-1-bromo-4-fluorobenzene (s14).

Colorless oil (purified by silica gel column chromatography, Hexane/EtOAc $=20 / 1$ ).

Yield: $586 \mathrm{mg}$ (72\%, synthesized from commercially available, 1-bromo-2-(bromomethyl)-4-fluorobenzene).

IR (neat) 3088, 3065, 3031, 2861, 2789, 1606, 1580, 1496, 1469, 1454, 1412, 1387, $1356,1308,1268,1220,1151,1114,1092,1030,958 \mathrm{~cm}^{-1}$.

${ }^{1} \mathrm{H}$ NMR $\left(300 \mathrm{MHz}, \mathrm{CDCl}_{3}\right) \delta 7.47(\mathrm{dd}, 1 \mathrm{H}, J=8.5,5.1 \mathrm{~Hz}), 7.44-7.27(\mathrm{~m}, 6 \mathrm{H}), 6.88$ (ddd, 1H, $J=8.1,8.1,3.0 \mathrm{~Hz}), 4.66(\mathrm{~s}, 2 \mathrm{H}), 4.58$ (s, 2H).

${ }^{13} \mathrm{C}$ NMR $\left(75 \mathrm{MHz}, \mathrm{CDCl}_{3}\right) \delta 162.2\left(\mathrm{~d}, J_{C-F}=244.8 \mathrm{~Hz}\right), 140.1\left(\mathrm{~d}, J_{C-F}=7.4 \mathrm{~Hz}\right), 137.7$, $133.4\left(\mathrm{~d}, J_{C-F}=8.0 \mathrm{~Hz}\right), 128.5,127.8,127.7,115.8\left(\mathrm{~d}, J_{C-F}=23.4 \mathrm{~Hz}\right), 115.8,115.7(\mathrm{~d}$, 
$\left.J_{C-F}=26.0 \mathrm{~Hz}\right), 72.9,71.0\left(\mathrm{~d}, J_{C-F}=1.2 \mathrm{~Hz}\right)$.

${ }^{19} \mathrm{~F} \mathrm{NMR}\left(283 \mathrm{MHz}, \mathrm{CDCl}_{3}\right) \delta 47.3(\mathrm{dd}, 1 \mathrm{~F}, J=9.0,13.4 \mathrm{~Hz})$.

Anal. Calcd for $\mathrm{C}_{14} \mathrm{H}_{12} \mathrm{BrFO}$ : C, 56.97; H, 4.10. Found: C, 56.77; H, 4.28.<smiles>O=Cc1ccc(F)cc1COCc1ccccc1</smiles>

2-((Benzyloxy)methyl)-4-fluorobenzaldehyde (s15).

Colorless oil (purified by silica gel column chromatography, Hexane/EtOAc $=8 / 1$ ).

Yield: 306 mg (69\%, synthesized from s14).

IR (neat) 3087, 3064, 3032, 2860, 2742, 1698, 1608, 1583, 1495, 1455, 1396, 1355, $1308,1273,1253,1236,1220,1195,1153,1113,1073,1028,969 \mathrm{~cm}^{-1}$.

${ }^{1} \mathrm{H}$ NMR $\left(300 \mathrm{MHz}, \mathrm{CDCl}_{3}\right) \delta 10.01(\mathrm{~s}, 1 \mathrm{H}), 7.86(\mathrm{dd}, 1 \mathrm{H}, J=8.4,5.7 \mathrm{~Hz}), 7.50$ (dd, $1 \mathrm{H}, J=9.9,2.7 \mathrm{~Hz}), 7.43-7.28(\mathrm{~m}, 5 \mathrm{H}), 7.14(\mathrm{ddd}, 1 \mathrm{H}, J=8.4,8.4$, $2.7 \mathrm{~Hz}), 5.00$ (s, $2 \mathrm{H}), 4.69$ (s, 2H).

${ }^{13} \mathrm{C} \mathrm{NMR}\left(75 \mathrm{MHz}, \mathrm{CDCl}_{3}\right) \delta 190.8,165.7\left(\mathrm{~d}, J_{C-F}=254.7 \mathrm{~Hz}\right), 144.7\left(\mathrm{~d}, J_{C-F}=9.3 \mathrm{~Hz}\right)$, $137.5,135.6\left(\mathrm{~d}, J_{C-F}=9.8 \mathrm{~Hz}\right), 129.2\left(\mathrm{~d}, J_{C-F}=3.1 \mathrm{~Hz}\right), 128.2,127.5,127.4,114.6(\mathrm{~d}$, $\left.J_{C-F}=24.0 \mathrm{~Hz}\right), 114.0\left(\mathrm{~d}, J_{C-F}=22.2 \mathrm{~Hz}\right), 72.6,68.6\left(\mathrm{~d}, J_{C-F}=1.2 \mathrm{~Hz}\right)$.

${ }^{19} \mathrm{~F} \mathrm{NMR}\left(283 \mathrm{MHz}, \mathrm{CDCl}_{3}\right) \delta 59.2(\mathrm{dd}, 1 \mathrm{~F}, J=9.1,15.8 \mathrm{~Hz})$.

Anal. Calcd for $\mathrm{C}_{15} \mathrm{H}_{13} \mathrm{FO}_{2}$ : C, 73.76; H, 5.36. Found: C, 73.53; H, 5.41.<smiles>COC(=O)C(=Cc1cccc(F)c1)C(=O)OCc1ccccc1</smiles>

Dimethyl 2-(2-((benzyloxy)methyl)-4-fluorobenzylidene)malonate (3g).

Colorless oil (purified by silica gel column chromatography, Hexane/EtOAc $=4 / 1$ ).

Yield: $339 \mathrm{mg}$ (82\%, synthesized from s15).

IR (neat) 3087, 3064, 3032, 3005, 2952, 2858, 1735, 1631, 1606, 1586, 1494, 1454, $1437,1363,1252,1222,1156,1101,1071,1028,985,964 \mathrm{~cm}^{-1}$.

${ }^{1} \mathrm{H}$ NMR (300 MHz, $\mathrm{CDCl}_{3}$ ) $\delta 7.97(\mathrm{~s}, 1 \mathrm{H}), 7.41-7.28(\mathrm{~m}, 6 \mathrm{H}), 7.21$ (dd, 1H, $J=8.4$, $2.7 \mathrm{~Hz}), 6.97$ (ddd, 1H, J = 8.4, 8.4, $2.7 \mathrm{~Hz}), 4.60$ (s, 2H), 4.58 (s, 2H), 3.85 (s, 3H), $3.72(\mathrm{~s}, 3 \mathrm{H})$.

${ }^{13} \mathrm{C}$ NMR $\left(75 \mathrm{MHz}, \mathrm{CDCl}_{3}\right) \delta 166.3,163.9,163.4\left(\mathrm{~d}, J_{C-F}=249.7 \mathrm{~Hz}\right), 140.3\left(\mathrm{~d}, J_{C-F}=\right.$ 
$7.4 \mathrm{~Hz}), 140.2,137.4,129.7\left(\mathrm{~d}, J_{C-F}=8.6 \mathrm{~Hz}\right), 128.3,128.0\left(\mathrm{~d}, J_{C-F}=3.1 \mathrm{~Hz}\right), 127.7$, $127.6,127.4\left(\mathrm{~d}, J_{C-F}=1.2 \mathrm{~Hz}\right), 115.6\left(\mathrm{~d}, J_{C-F}=22.2 \mathrm{~Hz}\right), 114.6\left(\mathrm{~d}, J_{C-F}=21.5 \mathrm{~Hz}\right)$, $72.5,69.1\left(\mathrm{~d}, J_{C-F}=1.9 \mathrm{~Hz}\right), 52.3,52.5$.

${ }^{19} \mathrm{~F} \mathrm{NMR}\left(283 \mathrm{MHz}, \mathrm{CDCl}_{3}\right) \delta 52.1(\mathrm{dd}, 1 \mathrm{~F}, J=9.3,13.9 \mathrm{~Hz})$.

Anal. Calcd for $\mathrm{C}_{20} \mathrm{H}_{19} \mathrm{FO}_{5}$ : C, 67.03; H, 5.34. Found: C, 67.12; H, 5.07.<smiles>Cc1cccc(COCc2ccccc2)c1I</smiles>

1-((Benzyloxy)methyl)-2-iodo-3-methylbenzene (s16).

Colorless oil (purified by silica gel column chromatography, Hexane/EtOAc $=20 / 1$ ).

Yield: $428 \mathrm{mg}$ (68\%, synthesized from 2-bromo-1-(bromomethyl)-3-methylbenzene ${ }^{5}$ ).

IR (neat) 3087, 3062, 3030, 2972, 2945, 2920, 2854, 1574, 1496, 1452, 1406, 1390, $1380,1247,1208,1167,1156,1114,1074,1028,1005,974 \mathrm{~cm}^{-1}$.

${ }^{1} \mathrm{H}$ NMR (300 MHz, $\mathrm{CDCl}_{3}$ ) $\delta$ 7.45-7.15 (m, 7H), 4.66 (s, 2H), 4.59 (s, 2H), 2.48 (s, $3 \mathrm{H})$.

${ }^{13} \mathrm{C}$ NMR $\left(75 \mathrm{MHz}, \mathrm{CDCl}_{3}\right) \delta 141.9,141.0,138.0,128.8,128.3,127.8,127.7,127.6$, $125.9,104.9,77.0,72.6,29.2$.

Anal. Calcd for $\mathrm{C}_{15} \mathrm{H}_{15} \mathrm{IO}$ : C, 53.27; H, 4.47. Found: C, 53.42; H, 4.74.<smiles>Cc1cccc(COCc2ccccc2)c1C=O</smiles>

2-((Benzyloxy)methyl)-6-methylbenzaldehyde (s17).

Colorless oil (purified by silica gel column chromatography, Hexane/EtOAc $=8 / 1$ ).

Yield: $207 \mathrm{mg}$ (65\%, synthesized from s16).

IR (neat) 3063, 3031, 2956, 2919, 2850, 2773, 1693, 1593, 1580, 1496, 1467, 1454, $1411,1380,1359,1350,1307,1289,1245,1191,1170,1097,1072,1028,974 \mathrm{~cm}^{-1}$.

${ }^{1} \mathrm{H}$ NMR $\left(300 \mathrm{MHz}, \mathrm{CDCl}_{3}\right) \delta 10.58(\mathrm{~s}, 1 \mathrm{H}), 7.52-7.23(\mathrm{~m}, 7 \mathrm{H}), 7.21(\mathrm{~d}, 1 \mathrm{H}, J=7.8$ $\mathrm{Hz}), 4.90$ (s, 2H), 4.63 (s, 2H), 2.64 (s, 3H).

${ }^{13} \mathrm{C}$ NMR $\left(75 \mathrm{MHz}, \mathrm{CDCl}_{3}\right) \delta 193.2,141.2,137.9,133.0,131.6,131.2,128.3,127.7$, 127.6, 126.4, 72.6, 70.1, 20.3.

Anal. Calcd for $\mathrm{C}_{16} \mathrm{H}_{16} \mathrm{O}_{2}$ : C, 79.97; H, 6.71. Found: C, 79.78; H, 6.50. 


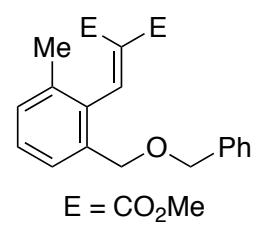

Dimethyl 2-(2-((benzyloxy)methyl)-6-methylbenzylidene)malonate (3h).

Colorless oil (purified by silica gel column chromatography, Hexane/EtOAc $=4 / 1$ ).

Yield: $177 \mathrm{mg}$ (63\%, synthesized from s17).

IR (neat) 3064, 3030, 3006, 2952, 2922, 2857, 1732, 1638, 1597, 1454, 1437, 1361, $1264,1222,1195,1072,1029,985 \mathrm{~cm}^{-1}$.

${ }^{1} \mathrm{H}$ NMR (300 MHz, $\left.\mathrm{CDCl}_{3}\right) \delta 8.00(\mathrm{~s}, 1 \mathrm{H}), 7.40-7.17(\mathrm{~m}, 7 \mathrm{H}), 7.13(\mathrm{~d}, 1 \mathrm{H}, J=7.2 \mathrm{~Hz})$, $4.52(\mathrm{~s}, 2 \mathrm{H}), 4.47(\mathrm{~s}, 2 \mathrm{H}), 3.87(\mathrm{~s}, 3 \mathrm{H}), 3.50(\mathrm{~s}, 3 \mathrm{H}), 2.22(\mathrm{~s}, 3 \mathrm{H})$.

${ }^{13} \mathrm{C}$ NMR $\left(75 \mathrm{MHz}, \mathrm{CDCl}_{3}\right) \delta 165.2,163.8,145.3,138.0,135.3,135.2,132.8,129.9$, $129.2,128.3,128.2,127.7,127.6,125.7,72.5,70.5,52.6,52.1,20.0$.

Anal. Calcd for $\mathrm{C}_{21} \mathrm{H}_{22} \mathrm{O}_{5}$ : C, 71.17; H, 6.26. Found: C, 71.06; H, 6.46.

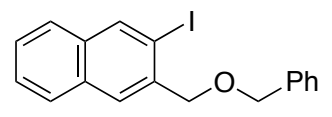

2-((Benzyloxy)methyl)-3-iodonaphthalene (s18).

Colorless amorphous (purified by silica gel column chromatography, Hexane/EtOAc = 20/1).

Yield: $629 \mathrm{mg}$ (55\%, synthesized from 2-bromomethyl-3-iode-naphthalene ${ }^{6}$ ).

IR (neat) 3054, 3030, 2912, 2855, 1585, 1495, 1453, 1355, 1269, 1204, 1169, 1129, $1095,1028,972,880 \mathrm{~cm}^{-1}$.

${ }^{1} \mathrm{H}$ NMR $\left(300 \mathrm{MHz}, \mathrm{CDCl}_{3}\right.$ ) $\delta 8.39$ (s, 1H), 7.92 (s, 1H), 7.87-7.77 (m, 1H), 7.76-7.66 (m, 1H), 7.55-7.28 (m, 7H), $4.72(\mathrm{~s}, 2 \mathrm{H}), 4.69(\mathrm{~s}, 2 \mathrm{H})$.

${ }^{13} \mathrm{C} \mathrm{NMR}\left(75 \mathrm{MHz}, \mathrm{CDCl}_{3}\right) \delta 138.4,137.9,136.1,134.1,132.6,128.4,127.8,127.8$, $127.7,127.4,126.6,126.5,126.3,95.0,75.9,72.7$.

Anal. Calcd for $\mathrm{C}_{18} \mathrm{H}_{15} \mathrm{IO}$ : C, 57.77; H, 4.04. Found: C, 57.50; H, 3.81 .

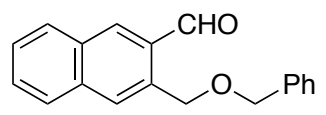

3-((Benzyloxy)methyl)-2-naphthaldehyde (s19). 
Colorless amorphous (purified by silica gel column chromatography, Hexane/EtOAc = $8 / 1)$.

Yield: $214 \mathrm{mg}$ (48\%, synthesized from s18).

IR (neat) 3059, 3030, 2857, 2798, 2742, 2720, 1693, 1631, 1596, 1496, 1467, 1454, $1358,1252,1174,1106,1072,1028,891 \mathrm{~cm}^{-1}$.

${ }^{1} \mathrm{H}$ NMR $\left(300 \mathrm{MHz}, \mathrm{CDCl}_{3}\right.$ ) $\delta 10.29$ (s, 1H), 8.38 (s, 1H), 8.09 (s, 1H), 8.00 (d, 1H, J = $8.1 \mathrm{~Hz}), 7.91(\mathrm{~d}, 1 \mathrm{H}, J=8.1 \mathrm{~Hz}), 7.69-7.54(\mathrm{~m}, 2 \mathrm{H}), 7.49-7.28(\mathrm{~m}, 5 \mathrm{H}), 5.13(\mathrm{~s}, 2 \mathrm{H})$, $4.74(\mathrm{~s}, 2 \mathrm{H})$.

${ }^{13} \mathrm{C}$ NMR $\left(75 \mathrm{MHz}, \mathrm{CDCl}_{3}\right) \delta 192.6,138.0,136.3,135.2,135.2,131.5,131.4,129.0$, $128.9,128.2,127.6,127.5,127.5,126.8,126.5,72.6,69.7$.

Anal. Calcd for $\mathrm{C}_{19} \mathrm{H}_{16} \mathrm{O}_{2}$ : C, 82.58; H, 5.84. Found: C, 82.76; H, 5.96.

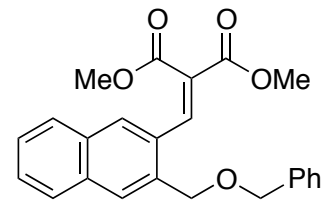

Dimethyl 2-((3-((benzyloxy)methyl)naphthalen-2-yl)methylene)malonate (3i).

Colorless amorphous (purified by silica gel column chromatography, Hexane/EtOAc = $4 / 1)$.

Yield: $258 \mathrm{mg}$ (86\%, synthesized from $\mathbf{s 1 9})$.

IR (neat) 3059, 3030, 3005, 2951, 2851, 1732, 1620, 1496, 1453, 1436, 1363, 1266, $1219,1182,1102,1070,892 \mathrm{~cm}^{-1}$.

${ }^{1} \mathrm{H}$ NMR $\left(300 \mathrm{MHz}, \mathrm{CDCl}_{3}\right) \delta 8.26(\mathrm{~s}, 1 \mathrm{H}), 7.88$ (s, 1H), 7.85 (s, 1H), 7.88-7.77 (m, 2H), 7.57-7.46 (m, 2H), 7.43-7.28 (m, 5H), 4.75 (s, 2H), 4.62 (s, 2H), $3.88(\mathrm{~s}, 3 \mathrm{H})$, $3.69(\mathrm{~s}, 3 \mathrm{H})$.

${ }^{13} \mathrm{C}$ NMR $\left(75 \mathrm{MHz}, \mathrm{CDCl}_{3}\right) \delta 166.7,164.1,142.0,127.7,133.8,133.5,132.5,130.8$, $128.3,128.2$, 128.1, 128.0, 127.8, 127.6, 127.6, 127.5, 127.3, 126.6, 72.3, 70.6, 52.5, 52.4 .

Anal. Calcd for $\mathrm{C}_{24} \mathrm{H}_{22} \mathrm{O}_{5}$ : C, 73.83; H, 5.68. Found: C, 74.08; H, 5.47.

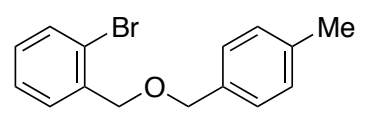

1-Bromo-2-(((4-methylbenzyl)oxy)methyl)benzene (s20).

Colorless oil (purified by silica gel column chromatography, Hexane/EtOAc $=20 / 1$ ). 
Yield: $647 \mathrm{mg}$ (95\%, synthesized from s1).

IR (neat) 3054, 3021, 2921, 2857, 1569, 1517, 1468, 1440, 1355, 1204, 1120, 1093, $1044,1024,940 \mathrm{~cm}^{-1}$.

${ }^{1} \mathrm{H}$ NMR (300 MHz, $\mathrm{CDCl}_{3}$ ) $\delta$ 7.58-7.47 (m, 2H), 7.36-7.25 (m, 3H), 7.22-7.08 (m, $3 \mathrm{H}), 4.61(\mathrm{~s}, 2 \mathrm{H}), 4.60(\mathrm{~s}, 2 \mathrm{H}), 2.35(\mathrm{~s}, 3 \mathrm{H})$.

${ }^{13} \mathrm{C}$ NMR $\left(75 \mathrm{MHz}, \mathrm{CDCl}_{3}\right) \delta 137.7,137.3,134.9,132.4,129.1,127.8,127.3,122.7$, 72.6, 71.3, 21.1.

Anal. Calcd for $\mathrm{C}_{15} \mathrm{H}_{15} \mathrm{BrO}$ : C, 61.87; H, 5.19. Found: C, 61.74; H, 5.43.

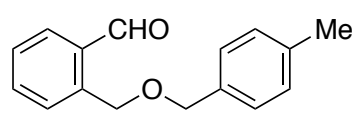

2-(((4-Methylbenzyl)oxy)methyl)benzaldehyde (s21).

Colorless oil (purified by silica gel column chromatography, Hexane/EtOAc $=8 / 1$ ).

Yield: $402 \mathrm{mg}$ (78\%, synthesized from s20).

IR (neat) 3050, 2024, 2922, 2858, 2735, 1696, 1601, 1575, 1517, 1454, 1355, 1200, $1118,1082,1021 \mathrm{~cm}^{-1}$.

${ }^{1} \mathrm{H}$ NMR $\left(300 \mathrm{MHz}, \mathrm{CDCl}_{3}\right) \delta 10.22(\mathrm{~s}, 1 \mathrm{H}), 7.86(\mathrm{dd}, 1 \mathrm{H}, J=7.5,1.5 \mathrm{~Hz}), 7.78(\mathrm{~d}, 1 \mathrm{H}$, $J=7.5 \mathrm{~Hz}), 7.59(\mathrm{ddd}, 1 \mathrm{H}, J=7.5,7.5,1.5 \mathrm{~Hz}), 7.47(\mathrm{dd}, 1 \mathrm{H}, J=7.5,7.5 \mathrm{~Hz}), 7.28(\mathrm{~d}$, $2 \mathrm{H}, J=7.8 \mathrm{~Hz}), 7.17$ (d, 2H, $J=7.8 \mathrm{~Hz}), 4.96(\mathrm{~s}, 2 \mathrm{H}), 4.62$ (s, 2H), 2.35 (s, 3H).

${ }^{13} \mathrm{C}$ NMR $\left(75 \mathrm{MHz}, \mathrm{CDCl}_{3}\right) \delta 192.6,140.8,137.3,134.8,133.7,133.3,132.1,129.0$, 128.2, 127.8, 127.6, 72.6, 69.2, 21.0.

Anal. Calcd for $\mathrm{C}_{16} \mathrm{H}_{16} \mathrm{O}_{2}$ : C, 79.97; H, 6.71. Found: C, 79.78; H, 6.85.

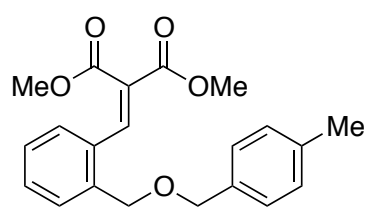

Dimethyl 2-(2-(((4-methylbenzyl)oxy)methyl)benzylidene)malonate (3j).

Colorless oil (purified by silica gel column chromatography, Hexane/EtOAc $=4 / 1$ ).

Yield: $381 \mathrm{mg}$ (73\%, synthesized from s21).

IR (neat) 3025, 3005, 2858, 1733, 1697, 1628, 1436, 1361, 1262, 1218, 1111, 1070, 986 $\mathrm{cm}^{-1}$.

${ }^{1} \mathrm{H}$ NMR $\left(300 \mathrm{MHz}, \mathrm{CDCl}_{3}\right) \delta 8.12(\mathrm{~s}, 1 \mathrm{H}), 7.42(\mathrm{dd}, 1 \mathrm{H}, J=7.5,7.5 \mathrm{~Hz}), 7.37-7.25(\mathrm{~m}$, $5 \mathrm{H}), 7.16(\mathrm{~d}, 2 \mathrm{H}, J=7.8 \mathrm{~Hz}), 4.58(\mathrm{~s}, 2 \mathrm{H}), 4.53(\mathrm{~s}, 2 \mathrm{H}), 3.86(\mathrm{~s}, 3 \mathrm{H}), 3.71(\mathrm{~s}, 3 \mathrm{H}), 2.35$ 
$(\mathrm{s}, 3 \mathrm{H})$.

${ }^{13} \mathrm{C}$ NMR $\left(75 \mathrm{MHz}, \mathrm{CDCl}_{3}\right) \delta 166.4,164.0,141.7,137.1,137.1,134.6,132.4,129.8$, $129.0,128.8,127.8,127.7,127.6,127.1,72.1,69.7,52.3,52.1,20.9$.

Anal. Calcd for $\mathrm{C}_{21} \mathrm{H}_{22} \mathrm{O}_{5}$ : C, 71.17; H, 6.26. Found: C, 71.04; H, 6.16.

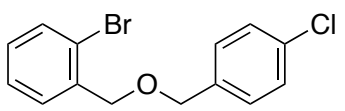

1-Bromo-2-(((4-chlorobenzyl)oxy)methyl)benzene (s22).

Colorless oil (purified by silica gel column chromatography, Hexane/EtOAc $=20 / 1$ ).

Yield: $560 \mathrm{mg}$ (87\%, synthesized from s1).

IR (neat) 3063, 2924, 2856, 1598, 1569, 1492, 1468, 1440, 1355, 1206, 1120, 1091, $1026,1015 \mathrm{~cm}^{-1}$.

${ }^{1} \mathrm{H}$ NMR (300 MHz, $\left.\mathrm{CDCl}_{3}\right) \delta 7.55(\mathrm{dd}, 1 \mathrm{H}, J=7.5,1.2 \mathrm{~Hz}), 7.50(\mathrm{dd}, 1 \mathrm{H}, J=7.5,1.5$ $\mathrm{Hz}), 7.38-7.25$ (m, 5H), 7.15 (ddd, 1H, J = 7.5, 7.5, 1.5 Hz), $4.62(\mathrm{~s}, 2 \mathrm{H}), 4.60$ (s, 2H).

${ }^{13} \mathrm{C}$ NMR $\left(75 \mathrm{MHz}, \mathrm{CDCl}_{3}\right) \delta 137.2,136.4,133.2,132.3,128.9,128.8,128.8,128.3$, $127.2,122.6,71.7,71.5$.

Anal. Calcd for $\mathrm{C}_{14} \mathrm{H}_{12} \mathrm{BrClO}$ : C, 53.96; H, 3.88. Found: C, 53.72; H, 4.13.<smiles>O=Cc1ccccc1COCc1ccc(Cl)cc1</smiles>

2-(((4-Chlorobenzyl)oxy)methyl)benzaldehyde (s23).

Colorless oil (purified by silica gel column chromatography, Hexane/EtOAc $=8 / 1$ ).

Yield: $310 \mathrm{mg}$ (69\%, synthesized from s22).

IR (neat) 3067, 3033, 2859, 2737, 1695, 1600, 1576, 1492, 1408, 1354, 1301, 1198, $1118,1088,1015 \mathrm{~cm}^{-1}$.

${ }^{1} \mathrm{H}$ NMR $\left(300 \mathrm{MHz}, \mathrm{CDCl}_{3}\right) \delta 10.20(\mathrm{~s}, 1 \mathrm{H}), 7.86(\mathrm{~d}, 1 \mathrm{H}, J=7.5 \mathrm{~Hz}), 7.68(\mathrm{~d}, 1 \mathrm{H}, J=$ $7.5 \mathrm{~Hz}), 7.61(\mathrm{ddd}, 1 \mathrm{H}, J=7.5,7.5,1.5 \mathrm{~Hz}), 7.48(\mathrm{dd}, 1 \mathrm{H}, J=7.5,7.5 \mathrm{~Hz}), 7.39-7.27$ (m, 4H), $4.98(\mathrm{~s}, 2 \mathrm{H}), 4.62(\mathrm{~s}, 2 \mathrm{H})$.

${ }^{13} \mathrm{C}$ NMR (75 MHz, $\left.\mathrm{CDCl}_{3}\right) \delta 192.4,192.4,140.3,136.4,133.6,133.1,132.3,128.7$, $128.3,127.9,127.5,71.7,69.4$.

Anal. Calcd for $\mathrm{C}_{15} \mathrm{H}_{13} \mathrm{ClO}_{2}$ : C, 69.10; H, 5.03. Found: C, 69.38; H, 4.79. 
<smiles>COC(=O)C(=Cc1ccccc1COCc1ccc(Cl)cc1)C(=O)OC</smiles>

Dimethyl 2-(2-(((4-chlorobenzyl)oxy)methyl)benzylidene)malonate (3k).

Colorless oil (purified by silica gel column chromatography, Hexane/EtOAc $=4 / 1$ ).

Yield: $307 \mathrm{mg}$ (71\%, synthesized from s23).

IR (neat) 3065, 3031, 3001, 2952, 2859, 1732, 1629, 1492, 1436, 1362, 1262, 1218, $1069,1014,943 \mathrm{~cm}^{-1}$.

${ }^{1} \mathrm{H}$ NMR (300 MHz, $\mathrm{CDCl}_{3}$ ) $\delta 8.10$ (s, 1H), 7.45-7.24 (m, 8H), 4.59 (s, 2H), 4.52 (s, $2 \mathrm{H}), 3.85(\mathrm{~s}, 3 \mathrm{H}), 3.71(\mathrm{~s}, 3 \mathrm{H})$.

${ }^{13} \mathrm{C}$ NMR $\left(75 \mathrm{MHz}, \mathrm{CDCl}_{3}\right) \delta 166.5,164.1,141.8,136.9,136.3,133.4,132.7,130.0$, $129.2,129.1,128.5,128.2,127.9,127.5,70.2,71.5,52.6,52.4$.

Anal. Calcd for $\mathrm{C}_{20} \mathrm{H}_{19} \mathrm{ClO}_{5}$ : C, 64.09; H, 5.11. Found: C, 64.32; H, 4.98.

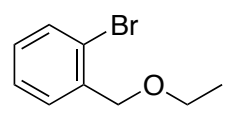

1-Bromo-2-(ethoxymethyl)benzene (s24).

Colorless oil (purified by silica gel column chromatography, Hexane/EtOAc $=20 / 1$ ).

Yield: 396 mg (92\%, synthesized from s1).

IR (neat) 3066, 2976, 2929, 2868, 1569, 1469, 1440, 1373, 1351, 1206, 1173, 1158, $1123,1102,1044,1028,895 \mathrm{~cm}^{-1}$.

${ }^{1} \mathrm{H}$ NMR (300 MHz, $\left.\mathrm{CDCl}_{3}\right) \delta 7.53(\mathrm{dd}, 1 \mathrm{H}, J=7.5,1.2 \mathrm{~Hz}), 7.49$ (d, $\left.1 \mathrm{H}, J=7.5 \mathrm{~Hz}\right)$, 7.31 (ddd, $1 \mathrm{H}, J=7.5,7.5,1.2 \mathrm{~Hz}), 7.14(\mathrm{ddd}, 1 \mathrm{H}, J=7.5,7.5,1.2 \mathrm{~Hz}), 4.57$ (s, 2H), $3.62(\mathrm{q}, 2 \mathrm{H}, J=7.2 \mathrm{~Hz}), 1.29(\mathrm{t}, 3 \mathrm{H}, J=7.2 \mathrm{~Hz})$.

${ }^{13} \mathrm{C}$ NMR $\left(75 \mathrm{MHz}, \mathrm{CDCl}_{3}\right) \delta 137.9,132.4,128.8,128.7,127.3,122.5,71.8,66.2,15.2$. Anal. Calcd for $\mathrm{C}_{9} \mathrm{H}_{11}$ BrO: C, 50.26; H, 5.15. Found: C, 50.03; H, 5.04.<smiles>CCOCc1ccccc1C=O</smiles>

2-(Ethoxymethyl)benzaldehyde (s25).

Colorless oil (purified by silica gel column chromatography, Hexane/EtOAc $=8 / 1$ ).

Yield: $287 \mathrm{mg}$ (96\%, synthesized from s24).

IR (neat) 2976, 2930, 2871, 2736, 1697, 1601, 1576, 1487, 1454, 1401, 1373, 1351, 
$1301,1287,1196,1121,1095,862 \mathrm{~cm}^{-1}$.

${ }^{1} \mathrm{H}$ NMR $\left(300 \mathrm{MHz}, \mathrm{CDCl}_{3}\right) \delta 10.24(\mathrm{~s}, 1 \mathrm{H}), 7.87(\mathrm{dd}, 1 \mathrm{H}, J=7.5,1.5 \mathrm{~Hz}), 7.65(\mathrm{~d}, 1 \mathrm{H}$, $J=7.5 \mathrm{~Hz}), 7.60(\mathrm{ddd}, 1 \mathrm{H}, J=7.5,7.5,1.5 \mathrm{~Hz}), 7.47(\mathrm{ddd}, 1 \mathrm{H}, J=7.5,7.5,1.5 \mathrm{~Hz})$, $4.92(\mathrm{~s}, 2 \mathrm{H}), 3.64(\mathrm{q}, 2 \mathrm{H}, J=7.2 \mathrm{~Hz}), 1.28(\mathrm{t}, 3 \mathrm{H}, J=7.2 \mathrm{~Hz})$.

${ }^{13} \mathrm{C}$ NMR $\left(75 \mathrm{MHz}, \mathrm{CDCl}_{3}\right) \delta 192.7,141.1,133.7,133.3,132.1,128.1,127.6,69.7,66.2$, 15.1 .

Anal. Calcd for $\mathrm{C}_{10} \mathrm{H}_{12} \mathrm{O}_{2}$ : C, 73.15; H, 7.37. Found: C, 73.24; H, 7.13.

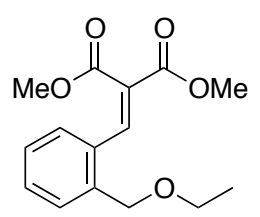

Dimethyl 2-(2-(ethoxymethyl)benzylidene)malonate (31).

Colorless oil (purified by silica gel column chromatography, Hexane/EtOAc $=4 / 1$ ).

Yield: $307 \mathrm{mg}$ (66\%, synthesized from s25).

IR (neat) 2976, 2953, 2870, 1733, 1630, 1437, 1374, 1263, 1218, 1092, 1069, 986, 944, $836 \mathrm{~cm}^{-1}$.

${ }^{1} \mathrm{H}$ NMR $\left(300 \mathrm{MHz}, \mathrm{CDCl}_{3}\right) \delta 8.12(\mathrm{~s}, 1 \mathrm{H}), 7.42(\mathrm{~d}, 1 \mathrm{H}, J=7.5 \mathrm{~Hz}), 7.39-7.31(\mathrm{~m}, 2 \mathrm{H})$, $7.28(\mathrm{dd}, 1 \mathrm{H}, J=7.5,1.5 \mathrm{~Hz}), 4.55(\mathrm{~s}, 2 \mathrm{H}), 3.86(\mathrm{~s}, 2 \mathrm{H}), 3.72(\mathrm{~s}, 2 \mathrm{H}), 3.57(\mathrm{q}, 2 \mathrm{H}, J=$ $7.2 \mathrm{~Hz}), 1.25(\mathrm{t}, 3 \mathrm{H}, J=7.2 \mathrm{~Hz})$.

${ }^{13} \mathrm{C}$ NMR $\left(75 \mathrm{MHz}, \mathrm{CDCl}_{3}\right) \delta 166.5,164.2,141.9,137.5,132.3,129.9,128.8,127.8$, $127.7,127.1,70.4,65.9,52.5,52.3,15.0$.

Anal. Calcd for $\mathrm{C}_{15} \mathrm{H}_{18} \mathrm{O}_{5}$ : C, 64.74; H, 6.52. Found: C, 64.44; H, 6.24. 


\section{Synthesis of [1,6]-hydride shift involved adducts.}

\section{General Procedure of the formation of benzoxepine derivatives.}

To a solution of benzylidene malonate $3(0.10 \mathrm{mmol})$ in $\mathrm{ClCH}_{2} \mathrm{CH}_{2} \mathrm{Cl}(1.0 \mathrm{~mL})$ was added $\mathrm{Yb}(\mathrm{OTf})_{3}(3.1-18.6 \mathrm{mg}, 5-30 \mathrm{~mol} \%)$, and the mixture was heated at reflux (heat block). After completion of the reaction, the reaction was stopped by adding saturated aqueous $\mathrm{NaHCO}_{3}$. The crude products were extracted with EtOAc (x3) and the combined organic extracts were washed with brine, dried $\left(\mathrm{Na}_{2} \mathrm{SO}_{4}\right)$, and concentrated in vacuo. The residue was purified by preparative TLC to give benzoxepines 5 .

\section{General Procedure of the formation of indane derivatives.}

To a solution of benzylidene malonate $3(0.10 \mathrm{mmol})$ in $\mathrm{ClCH}_{2} \mathrm{CH}_{2} \mathrm{Cl}(1.0 \mathrm{~mL})$ was added $\mathrm{Sc}(\mathrm{OTf})_{3}(2.5-9.8 \mathrm{mg}, 5-20 \mathrm{~mol} \%)$, and the mixture was heated at reflux (heat block). After completion of the reaction, the reaction was stopped by adding saturated aqueous $\mathrm{NaHCO}_{3}$. The crude products were extracted with EtOAc (x3) and the combined organic extracts were washed with brine, dried $\left(\mathrm{Na}_{2} \mathrm{SO}_{4}\right)$, and concentrated in vacuo. The residue was purified by preparative TLC to give indane derivatives 6 .

\section{Procedure of the formation of the formation of benzoxepine $5 \mathrm{a}$ (1.20 $\mathrm{mmol}$ scale).}

To a solution of benzylidene malonate $3 \mathbf{a}(408.2 \mathrm{mg}, 1.20 \mathrm{mmol})$ in $\mathrm{ClCH}_{2} \mathrm{CH}_{2} \mathrm{Cl}$ (12.0 $\mathrm{mL}$ ) was added $\mathrm{Yb}(\mathrm{OTf})_{3}(74.4 \mathrm{mg}, 10 \mathrm{~mol} \%)$, and the mixture was heated at reflux (oil bath). After completion of the reaction, the reaction was stopped by adding saturated aqueous $\mathrm{NaHCO}_{3}$. The crude products were extracted with EtOAc (x3) and the combined organic extracts were washed with brine, dried $\left(\mathrm{Na}_{2} \mathrm{SO}_{4}\right)$, and concentrated in vacuo. The residue was purified by column chromatography (silica gel, hexane $/$ EtOAc $=5 / 1)$ to give benzoxepines $\mathbf{5 a}(378.9 \mathrm{mg}, 93 \%)$.

\section{Procedure of the formation of the formation of indane 6 a $(1.20 \mathrm{mmol}$ scale).}

To a solution of benzylidene malonate $3 \mathbf{a}(408.0 \mathrm{mg}, 1.20 \mathrm{mmol})$ in $\mathrm{ClCH}_{2} \mathrm{CH}_{2} \mathrm{Cl}(12.0$ $\mathrm{mL}$ ) was added $\mathrm{Sc}(\mathrm{OTf})_{3}(59.0 \mathrm{mg}, 10 \mathrm{~mol} \%$ ), and the mixture was heated at reflux (oil bath). After completion of the reaction, the reaction was stopped by adding saturated aqueous $\mathrm{NaHCO}_{3}$. The crude products were extracted with EtOAc (x3) and the combined organic extracts were washed with brine, dried $\left(\mathrm{Na}_{2} \mathrm{SO}_{4}\right)$, and concentrated in vacuo. The residue was purified by column chromatography (silica gel, 
hexane/EtOAc $=8 / 1)$ to give indane $6 \mathbf{a}(221.7 \mathrm{mg}, 79 \%)$.

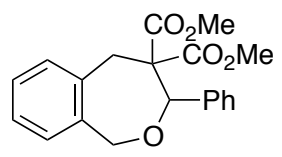

Dimethyl 3-phenyl-3,5-dihydrobenzo[c]oxepine-4,4(1H)-dicarboxylate (5a).

Colorless oil (purified by preparative TLC, Hexane/EtOAc $=4 / 1$ ).

Yield: $31.8 \mathrm{mg}(98 \%)$.

IR (neat) 3062, 3031, 2951, 2851, 1731, 1495, 1449, 1434, 1365, 1260, 1227, 1203, $1176,1092,1055,1039,975,946 \mathrm{~cm}^{-1}$.

${ }^{1} \mathrm{H}$ NMR (300 MHz, $\left.\mathrm{CDCl}_{3}\right) \delta$ 7.48-7.40 (m, 2H), 7.35-7.12 (m, 5H), 7.11-7.00 (m, $2 \mathrm{H}), 5.43(\mathrm{~s}, 1 \mathrm{H}), 5.38(\mathrm{~d}, 1 \mathrm{H}, J=15.3 \mathrm{~Hz}), 5.01(\mathrm{~d}, 1 \mathrm{H}, J=15.3 \mathrm{~Hz}), 4.15(\mathrm{~d}, 1 \mathrm{H}, J=$ $14.1 \mathrm{~Hz}), 3.57(\mathrm{~s}, 3 \mathrm{H}), 3.23(\mathrm{~d}, 1 \mathrm{H}, J=14.1 \mathrm{~Hz}), 3.20(\mathrm{~s}, 3 \mathrm{H})$.

${ }^{13} \mathrm{C}$ NMR $\left(75 \mathrm{MHz}, \mathrm{CDCl}_{3}\right) \delta 170.8,168.9,139.1,137.2,134.2,130.7,127.7,127.6$, 127.2, 127.1, 125.9, 81.2, 74.5, 66.0, 52.3, 51.8, 37.5.

Anal. Calcd for $\mathrm{C}_{20} \mathrm{H}_{20} \mathrm{O}_{5}$ : C, 70.57; H, 5.92. Found: C, 70.34; H, 6.20.

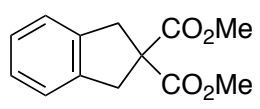

Dimethyl $1 H$-indene-2,2(3H)-dicarboxylate (6a).

Colorless oil (purified by preparative TLC, Hexane/EtOAc $=4 / 1$ ).

Yield: $21.8 \mathrm{mg}$ (98\% from 3a), $15.9 \mathrm{mg}$ (70\% from 3j), and $19.0 \mathrm{mg}$ (83\% from 3k).

IR (neat) 3075, 3007, 2957, 2860, 1736, 1449, 1437, 1277, 1244, 1201, 1170, 1154, $1074,870 \mathrm{~cm}^{-1}$.

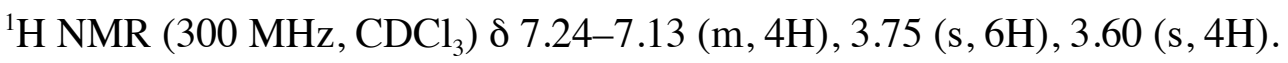

${ }^{13} \mathrm{C}$ NMR $\left(75 \mathrm{MHz}, \mathrm{CDCl}_{3}\right) \delta 172.1,139.8,126.9,124.2,60.2,52.9,40.5$.

Anal. Calcd for $\mathrm{C}_{13} \mathrm{H}_{14} \mathrm{O}_{4}$ : C, 66.66; H, 6.02. Found: C, 66.48; H, 5.76.

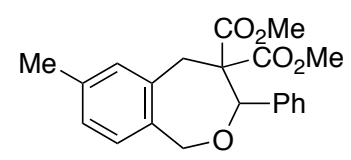

Dimethyl 7-methyl-3-phenyl-3,5-dihydrobenzo[c]oxepine-4,4(1H)-dicarboxylate (5b).

Colorless oil (purified by preparative TLC, Hexane/EtOAc $=4 / 1$ ). 
Yield: $28.6 \mathrm{mg}(79 \%)$.

IR (neat) 3032, 3008, 2951, 2925, 2855, 1731, 1615, 1506, 1495, 1449, 1434, 1264, $1204,1092,1055,809 \mathrm{~cm}^{-1}$.

${ }^{1} \mathrm{H}$ NMR (300 MHz, $\mathrm{CDCl}_{3}$ ) $\delta$ 7.48-7.37 (m, 2H), 7.35-7.17 (m, 3H), $7.02(\mathrm{~d}, 1 \mathrm{H}, J=$ $7.8 \mathrm{~Hz}), 6.93(\mathrm{~d}, 1 \mathrm{H}, J=7.8 \mathrm{~Hz}), 6.89(\mathrm{~s}, 1 \mathrm{H}), 5.39(\mathrm{~s}, 1 \mathrm{H}), 5.25(\mathrm{~d}, 1 \mathrm{H}, J=15.0 \mathrm{~Hz})$, $4.96(\mathrm{~d}, 1 \mathrm{H}, J=15.0 \mathrm{~Hz}), 4.11(\mathrm{~d}, 1 \mathrm{H}, J=14.4 \mathrm{~Hz}), 3.58$ (s, 3H), 3.19 (s, 3H), 3.17 (d, $1 \mathrm{H}, J=14.4 \mathrm{~Hz}), 2.30(\mathrm{~s}, 3 \mathrm{H})$.

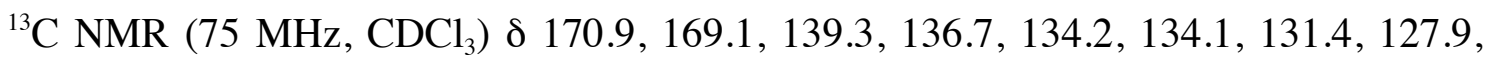
127.7, 127.6, 127.2, 126.0, 81.1, 74.4, 66.0, 52.3, 51.8, 37.6, 20.9.

Anal. Calcd for $\mathrm{C}_{21} \mathrm{H}_{22} \mathrm{O}_{5}$ : C, 71.17; H, 6.26. Found: C, 70.89; H, 6.43.

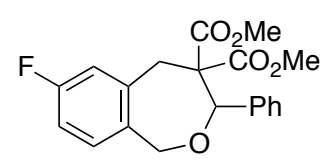

Dimethyl 7-fluoro-3-phenyl-3,5-dihydrobenzo[c]oxepine-4,4(1H)-dicarboxylate (5d).

Colorless oil (purified by preparative TLC, Hexane/EtOAc $=4 / 1$ ).

Yield: $33.0 \mathrm{mg}(89 \%)$.

IR (neat) 3063, 3033, 3004, 2952, 2860, 1731, 1614, 1595, 1500, 1450, 1434, 1259, $1234,1205,1175,1093,969,928,897 \mathrm{~cm}^{-1}$.

${ }^{1} \mathrm{H}$ NMR $\left(300 \mathrm{MHz}, \mathrm{CDCl}_{3}\right) \delta$ 7.46-7.39 (m, 2H), 7.35-7.22 (m, 3H), $7.00(\mathrm{dd}, 1 \mathrm{H}, J=$ $8.4,6.0 \mathrm{~Hz}), 6.91(\mathrm{~d}, 1 \mathrm{H}, J=8.4,8.4,2.4 \mathrm{~Hz}), 6.81(\mathrm{dd}, 1 \mathrm{H}, J=8.4,2.4 \mathrm{~Hz}), 5.41(\mathrm{~s}$, $1 \mathrm{H}), 5.22(\mathrm{~d}, 1 \mathrm{H}, J=15.0 \mathrm{~Hz}), 4.97(\mathrm{~d}, 1 \mathrm{H}, J=15.0 \mathrm{~Hz}), 4.12(\mathrm{~d}, 1 \mathrm{H}, J=14.1 \mathrm{~Hz})$, 3.59 (s, 3H), $3.20(\mathrm{~d}, 1 \mathrm{H}, J=14.1 \mathrm{~Hz}), 3.21(\mathrm{~s}, 3 \mathrm{H})$.

${ }^{13} \mathrm{C}$ NMR $\left(75 \mathrm{MHz}, \mathrm{CDCl}_{3}\right) \delta$ 170.7, 168.7, $161.7\left(\mathrm{~d}, J_{C-F}=243.5 \mathrm{~Hz}\right), 139.0,136.8(\mathrm{~d}$, $\left.J_{C-F}=7.4 \mathrm{~Hz}\right), 133.1\left(\mathrm{~d}, J_{C-F}=3.1 \mathrm{~Hz}\right), 127.9,127.7\left(\mathrm{~d}, J_{C-F}=8.6 \mathrm{~Hz}\right), 127.7,127.1$, $117.4\left(\mathrm{~d}, J_{C-F}=21.6 \mathrm{~Hz}\right), 114.0\left(\mathrm{~d}, J_{C-F}=21.0 \mathrm{~Hz}\right), 81.6,73.9,65.7,52.5,51.9,37.4$.

${ }^{19} \mathrm{~F}$ NMR $\left(283 \mathrm{MHz}, \mathrm{CDCl}_{3}\right) \delta 45.7(\mathrm{dd}, J=6.8,13.3 \mathrm{~Hz})$.

Anal. Calcd for $\mathrm{C}_{20} \mathrm{H}_{19} \mathrm{FO}_{5}$ : C, 67.03; H, 5.34. Found: C, 66.89; H, 5.47.

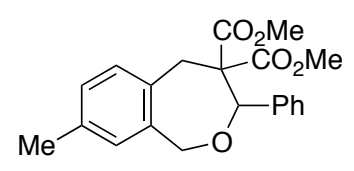

Dimethyl 8-methyl-3-phenyl-3,5-dihydrobenzo[c]oxepine-4,4(1H)-dicarboxylate (5e). 
Colorless oil (purified by preparative TLC, Hexane/EtOAc $=4 / 1$ ).

Yield: $33.5 \mathrm{mg}(94 \%)$.

IR (neat) 3032, 3004, 2951, 2925, 2858, 1731, 1506, 1495, 1448, 1434, 1266, 1221, $1200,1177,1096,1052,893 \mathrm{~cm}^{-1}$.

${ }^{1} \mathrm{H}$ NMR $\left(300 \mathrm{MHz}, \mathrm{CDCl}_{3}\right) \delta$ 7.48-7.38 (m, 2H), 7.37-7.18 (m, 3H), 7.03-6.95 (m, $2 \mathrm{H}), 6.85(\mathrm{~s}, 1 \mathrm{H}), 5.40(\mathrm{~s}, 1 \mathrm{H}), 5.24(\mathrm{~d}, 1 \mathrm{H}, J=15.0 \mathrm{~Hz}), 4.95(\mathrm{~d}, 1 \mathrm{H}, J=15.0 \mathrm{~Hz})$, $4.10(\mathrm{~d}, 1 \mathrm{H}, J=14.1 \mathrm{~Hz}), 3.57$ (s, 3H), 3.19 (d, 1H, J = 14.1 Hz), 3.19 (s, 3H), 2.29 (s, $3 \mathrm{H})$.

${ }^{13} \mathrm{C}$ NMR $\left(75 \mathrm{MHz}, \mathrm{CDCl}_{3}\right) \delta 171.0,169.1,139.3,137.1,136.9,131.1,130.7,127.9$, $127.7,127.7,127.2,126.7,81.3,74.6,66.1,52.3,51.8,37.2,20.9$.

Anal. Calcd for $\mathrm{C}_{21} \mathrm{H}_{22} \mathrm{O}_{5}$ : C, 71.17; H, 6.26. Found: C, 71.10; H, 6.07.

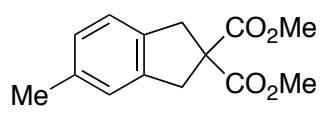

Dimethyl 5-methyl-1H-indene-2,2(3H)-dicarboxylate (6b).

Colorless oil (purified by preparative TLC, Hexane/EtOAc $=4 / 1$ ).

Yield: $22.0 \mathrm{mg}$ (89\% from 3b), and $19.4 \mathrm{mg}$ (79\% from 3e).

IR (neat) 3009, 2953, 2922, 2862, 1736, 1618, 1496, 1434, 1281, 1252, 1198, 1160, $1069,1052,881 \mathrm{~cm}^{-1}$.

${ }^{1} \mathrm{H}$ NMR $\left(300 \mathrm{MHz}, \mathrm{CDCl}_{3}\right) \delta 7.07(\mathrm{~d}, 1 \mathrm{H}, J=7.5 \mathrm{~Hz}), 7.01(\mathrm{~s}, 1 \mathrm{H}), 6.98(\mathrm{~d}, 1 \mathrm{H}, J=$ $7.5 \mathrm{~Hz}), 3.74(\mathrm{~s}, 6 \mathrm{H}), 3.55(\mathrm{~s}, 4 \mathrm{H}), 2.30(\mathrm{~s}, 3 \mathrm{H})$.

${ }^{13} \mathrm{C}$ NMR $\left(75 \mathrm{MHz}, \mathrm{CDCl}_{3}\right) \delta 172.1,139.9,136.7,136.6,127.7,124.8,123.9,60.5,52.9$, $40.5,40.2,21.2$.

Anal. Calcd for $\mathrm{C}_{14} \mathrm{H}_{16} \mathrm{O}_{4}$ : C, 67.73; H, 6.50. Found: C, 67.94; H, 6.78.

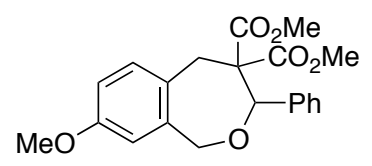

Dimethyl 8-methyl-3-phenyl-3,5-dihydrobenzo[c]oxepine-4,4(1H)-dicarboxylate (5f).

Colorless oil (purified by preparative TLC, Hexane/EtOAc $=4 / 1$ ).

Yield: $22.8 \mathrm{mg}(63 \%)$.

IR (neat) 3061, 3032, 3001, 2952, 2839, 1730, 1612, 1584, 1505, 1453, 1434, 1362, $1269,1222,1095,1043,980,983 \mathrm{~cm}^{-1}$. 
${ }^{1} \mathrm{H}$ NMR (300 MHz, $\mathrm{CDCl}_{3}$ ) $\delta$ 7.48-7.38 (m, 2H), 7.34-7.18 (m, 3H), 6.99 (d, 1H, $J=$ $8.4 \mathrm{~Hz}), 6.70(\mathrm{dd}, 1 \mathrm{H}, J=8.4,2.4 \mathrm{~Hz}), 6.56(\mathrm{~d}, 1 \mathrm{H}, J=2.4 \mathrm{~Hz}), 5.41(\mathrm{~s}, 1 \mathrm{H}), 5.24(\mathrm{~d}$, $1 \mathrm{H}, J=15.3 \mathrm{~Hz}), 4.96(\mathrm{~d}, 1 \mathrm{H}, J=15.3 \mathrm{~Hz}), 4.10(\mathrm{~d}, 1 \mathrm{H}, J=14.1 \mathrm{~Hz}), 3.77(\mathrm{~s}, 3 \mathrm{H}), 3.58$ (s, 3H), $3.19(\mathrm{~s}, 3 \mathrm{H}), 3.17(\mathrm{~d}, 1 \mathrm{H}, J=14.1 \mathrm{~Hz})$.

${ }^{13} \mathrm{C} \mathrm{NMR}\left(75 \mathrm{MHz}, \mathrm{CDCl}_{3}\right) \delta 171.0,169.1,158.7,139.2,138.6,131.9,127.8,127.6$, $127.1,126.2,112.6,111.2,81.3,74.6,66.2,55.1,52.3,51.8,36.7$.

Anal. Calcd for $\mathrm{C}_{21} \mathrm{H}_{22} \mathrm{O}_{5}$ : C, 71.17; H, 6.26. Found: C, 71.22; $\mathrm{H}, 6.35$.

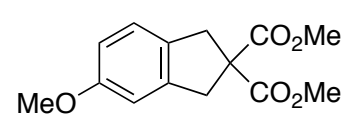

Dimethyl 5-methoxy-1H-indene-2,2(3H)-dicarboxylate (6c).

Colorless oil (purified by preparative TLC, Hexane/EtOAc $=4 / 1$ ).

Yield: $19.3 \mathrm{mg}$ (72\% from 3c), and $15.3 \mathrm{mg}$ (56\% from $\mathbf{3 f}$ ).

IR (neat) 3001, 2954, 2914, 2837, 1736, 1612, 1589, 1495, 1435, 1248, 1200, 1161, $1070,1032,875 \mathrm{~cm}^{-1}$.

${ }^{1} \mathrm{H}$ NMR $\left(300 \mathrm{MHz}, \mathrm{CDCl}_{3}\right) \delta 7.08(\mathrm{~d}, 1 \mathrm{H}, J=7.8 \mathrm{~Hz}), 6.74(\mathrm{~s}, 1 \mathrm{H}), 6.72(\mathrm{~d}, 1 \mathrm{H}, J=$ $7.8 \mathrm{~Hz}), 3.77$ (s, 3H), $3.74(\mathrm{~s}, 6 \mathrm{H}), 3.56(\mathrm{~s}, 2 \mathrm{H}), 3.52(\mathrm{~s}, 2 \mathrm{H})$.

${ }^{13} \mathrm{C}$ NMR $\left(75 \mathrm{MHz}, \mathrm{CDCl}_{3}\right) \delta 172.1,159.2,141.3,131.7,124.7,113.1,109.5,60.8,55.4$, $52.9,40.7,39.8$.

Anal. Calcd for $\mathrm{C}_{14} \mathrm{H}_{16} \mathrm{O}_{5}$ : C, 63.63; H, 6.10. Found: C, 63.76; H, 6.17.

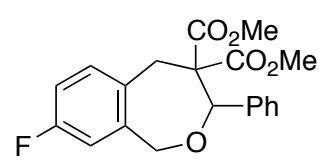

Dimethyl 8-fluoro-3-phenyl-3,5-dihydrobenzo[c]oxepine-4,4(1H)-dicarboxylate (5g).

Colorless oil (purified by preparative TLC, Hexane/EtOAc $=4 / 1$ ).

Yield: $27.5 \mathrm{mg}(72 \%)$.

IR (neat) 3064, 3032, 3004, 2952, 2925, 2848, 1731, 1616, 1499, 1447, 1435, 1257 , $1220,1201,1095,982,942,894 \mathrm{~cm}^{-1}$.

${ }^{1} \mathrm{H}$ NMR $\left(300 \mathrm{MHz}, \mathrm{CDCl}_{3}\right) \delta$ 7.46-7.39 (m, 3H), 7.35-7.20 (m, 5H), $7.05(\mathrm{dd}, 1 \mathrm{H}, J=$ 8.4, $5.7 \mathrm{~Hz}), 6.86(\mathrm{~d}, 1 \mathrm{H}, J=8.4,8.4,2.4 \mathrm{~Hz}), 6.75(\mathrm{dd}, 1 \mathrm{H}, J=8.4,2.4 \mathrm{~Hz}), 5.44(\mathrm{~s}$, $1 \mathrm{H}), 5.23(\mathrm{~d}, 1 \mathrm{H}, J=15.6 \mathrm{~Hz}), 4.96(\mathrm{~d}, 1 \mathrm{H}, J=15.6 \mathrm{~Hz}), 4.11(\mathrm{~d}, 1 \mathrm{H}, J=14.1 \mathrm{~Hz})$, $3.58(\mathrm{~s}, 3 \mathrm{H}), 3.20(\mathrm{~d}, 1 \mathrm{H}, J=14.1 \mathrm{~Hz}), 3.19(\mathrm{~s}, 3 \mathrm{H})$. 
${ }^{13} \mathrm{C} \mathrm{NMR}\left(75 \mathrm{MHz}, \mathrm{CDCl}_{3}\right) \delta 170.8,168.9,161.9\left(\mathrm{~d}, J_{C-F}=244.7 \mathrm{~Hz}\right), 139.6\left(\mathrm{~d}, J_{C-F}=\right.$ $6.8 \mathrm{~Hz}), 138.9,132.4\left(\mathrm{~d}, J_{C-F}=8.0 \mathrm{~Hz}\right), 129.9\left(\mathrm{~d}, J_{C-F}=3.1 \mathrm{~Hz}\right), 113.9\left(\mathrm{~d}, J_{C-F}=21.0\right.$ $\mathrm{Hz}), 112.8\left(\mathrm{~d}, J_{C-F}=21.6 \mathrm{~Hz}\right), 127.1,127.8,127.9,81.4,74.1\left(\mathrm{~d}, J_{C-F}=1.8 \mathrm{~Hz}\right), 66.1$, $52.5,51.9,36.5$.

${ }^{19} \mathrm{~F}$ NMR $\left(283 \mathrm{MHz}, \mathrm{CDCl}_{3}\right) \delta 46.3(\mathrm{dd}, J=7.1,13.9 \mathrm{~Hz})$.

Anal. Calcd for $\mathrm{C}_{20} \mathrm{H}_{19} \mathrm{FO}_{5}$ : C, 67.03; H, 5.34. Found: C, 67.25; H, 5.22.

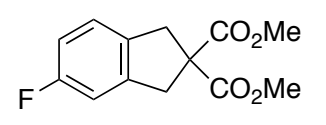

Dimethyl 5-fluoro-1H-indene-2,2(3H)-dicarboxylate (6d).

Colorless oil (purified by preparative TLC, Hexane/EtOAc $=4 / 1$ ).

Yield: $23.5 \mathrm{mg}$ (90\% from 3d), and $12.5 \mathrm{mg}$ (47\% from 3g).

IR (neat) 3071, 3009, 2975, 2956, 2924, 2903, 2852, 1737, 1614, 1598, 1487, 1443, $1434,1291,1248,1213,1182,1164,1078,1051,889 \mathrm{~cm}^{-1}$.

${ }^{1} \mathrm{H}$ NMR $\left(300 \mathrm{MHz}, \mathrm{CDCl}_{3}\right) \delta 7.11(\mathrm{dd}, 1 \mathrm{H}, J=8.1,5.7 \mathrm{~Hz}), 6.92-6.80(\mathrm{~m}, 2 \mathrm{H}), 3.75$ (s, $6 \mathrm{H}), 3.58(\mathrm{~s}, 2 \mathrm{H}), 3.54(\mathrm{~s}, 2 \mathrm{H})$.

${ }^{13} \mathrm{C} \mathrm{NMR}\left(75 \mathrm{MHz}, \mathrm{CDCl}_{3}\right) \delta 171.8,162.4\left(\mathrm{~d}, J_{C-F}=241.7 \mathrm{~Hz}\right), 141.9\left(\mathrm{~d}, J_{C-F}=8.0 \mathrm{~Hz}\right)$, $135.2\left(\mathrm{~d}, J_{C-F}=2.5 \mathrm{~Hz}\right), 125.1\left(\mathrm{~d}, J_{C-F}=8.6 \mathrm{~Hz}\right), 113.9\left(\mathrm{~d}, J_{C-F}=22.2 \mathrm{~Hz}\right), 111.3\left(\mathrm{~d}, J_{C-}\right.$ $\left.{ }_{F}=22.2 \mathrm{~Hz}\right), 60.8,53.0,40.5\left(\mathrm{~d}, J_{C-F}=1.9 \mathrm{~Hz}\right), 39.7$.

${ }^{19} \mathrm{~F}$ NMR (283 MHz, $\left.\mathrm{CDCl}_{3}\right) \delta 45.2(\mathrm{~m})$.

Anal. Calcd for $\mathrm{C}_{13} \mathrm{H}_{13} \mathrm{FO}_{4}$ : C, 61.90; H, 5.19. Found: C, 62.04; H, 4.96 .

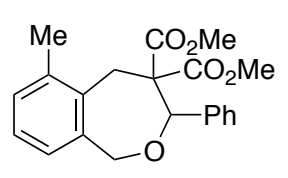

Dimethyl 6-methyl-3-phenyl-3,5-dihydrobenzo[c]oxepine-4,4(1H)-dicarboxylate (5h).

Colorless oil (purified by preparative TLC, Hexane/EtOAc $=4 / 1$ ).

Yield: $29.4 \mathrm{mg}(86 \%)$.

IR (neat) 3063, 3031, 3002, 2951, 2862, 1731, 1453, 1434, 1266, 1226, 1200, 1176, $1087,947,879 \mathrm{~cm}^{-1}$.

${ }^{1} \mathrm{H}$ NMR $\left(300 \mathrm{MHz}, \mathrm{CDCl}_{3}\right) \delta 7.42(\mathrm{~d}, 2 \mathrm{H}, J=7.5 \mathrm{~Hz}), 7.35-7.20(\mathrm{~m}, 3 \mathrm{H}), 7.13-7.04$ $(\mathrm{m}, 2 \mathrm{H}), 6.95-6.85(\mathrm{~m}, 1 \mathrm{H}), 5.43(\mathrm{~s}, 1 \mathrm{H}), 5.21(\mathrm{~d}, 1 \mathrm{H}, J=15.0 \mathrm{~Hz}), 4.98(\mathrm{~d}, 1 \mathrm{H}, J=$ $15.0 \mathrm{~Hz}), 3.96(\mathrm{~d}, 1 \mathrm{H}, J=14.4 \mathrm{~Hz}), 3.51(\mathrm{~d}, 1 \mathrm{H}, J=14.4 \mathrm{~Hz}), 3.54(\mathrm{~s}, 3 \mathrm{H}), 3.22(\mathrm{~s}, 3 \mathrm{H})$, 
$2.34(\mathrm{~s}, 3 \mathrm{H})$.

${ }^{13} \mathrm{C}$ NMR $\left(75 \mathrm{MHz}, \mathrm{CDCl}_{3}\right) \delta 170.9,169.1,139.3,137.5,137.1,132.8,129.5,127.8$, $127.6,127.2,126.7,124.4,81.6,74.6,65.2,52.2,51.7,32.0,19.8$.

Anal. Calcd for $\mathrm{C}_{21} \mathrm{H}_{22} \mathrm{O}_{5}$ : C, 71.17; H, 6.26. Found: C, 71.28; H, 6.49.

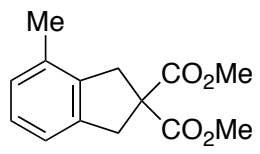

Dimethyl 4-methyl-1H-indene-2,2(3H)-dicarboxylate (6h).

Colorless oil (purified by preparative TLC, Hexane/EtOAc $=4 / 1$ ).

Yield: $26.1 \mathrm{mg}(97 \%)$.

IR (neat) 3024, 2953, 2919, 2847, 1737, 1603, 1434, 1281, 1250, 1200, 1172, 1161, $1067,960,866 \mathrm{~cm}^{-1}$.

${ }^{1} \mathrm{H}$ NMR $\left(300 \mathrm{MHz}, \mathrm{CDCl}_{3}\right) \delta$ 7.12-6.92 (m, 4H), 3.75 (s, 6H), 3.61 (s, 2H), 3.53 (s, $2 \mathrm{H}), 2.25(\mathrm{~s}, 3 \mathrm{H})$.

${ }^{13} \mathrm{C} \mathrm{NMR}\left(75 \mathrm{MHz}, \mathrm{CDCl}_{3}\right) \delta 172.2,139.5,138.7,133.7,127.7,127.2,121.4,59.7,52.9$, 40.7, 39.4, 18.9.

Anal. Calcd for $\mathrm{C}_{14} \mathrm{H}_{16} \mathrm{O}_{4}$ : C, 67.73; H, 6.50. Found: C, 67.47; H, 6.37.

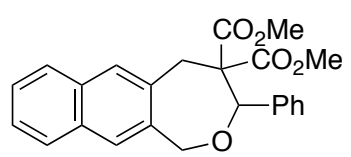

Dimethyl 3-phenyl-3,5-dihydronaphtho[2,3-c]oxepine-4,4(1H)-dicarboxylate (5i).

Colorless amorphous (purified by preparative TLC, Hexane/EtOAc $=4 / 1$ ).

Yield: $31.6 \mathrm{mg}(86 \%)$.

IR (neat) 3060, 3032, 3006, 2952, 2854, 1730, 1433, 1250, 1225, 1199, 1179, 1156, $1111,1078,1059,979,894 \mathrm{~cm}^{-1}$.

${ }^{1} \mathrm{H}$ NMR (300 MHz, $\left.\mathrm{CDCl}_{3}\right) \delta$ 7.80-7.72 (m, 2H), $7.56(\mathrm{~s}, 2 \mathrm{H}), 7.49-7.40(\mathrm{~m}, 4 \mathrm{H})$, 7.34-7.21 (m, 3H), $5.43(\mathrm{~d}, 1 \mathrm{H}, J=15.0 \mathrm{~Hz}), 5.40(\mathrm{~s}, 1 \mathrm{H}), 5.15(\mathrm{~d}, 1 \mathrm{H}, J=15.0 \mathrm{~Hz})$, $4.23(\mathrm{~d}, 1 \mathrm{H}, J=14.1 \mathrm{~Hz}), 3.57(\mathrm{~s}, 3 \mathrm{H}), 3.47(\mathrm{~d}, 1 \mathrm{H}, J=14.1 \mathrm{~Hz}), 3.23(\mathrm{~s}, 3 \mathrm{H})$.

${ }^{13} \mathrm{C}$ NMR $\left(100 \mathrm{MHz}, \mathrm{CDCl}_{3}\right) \delta$ 170.6, 168.9, 139.2, 135.4, 132.9, 132.7, 132.5, 129.1, $127.8,127.6,127.3,127.2$, 127.2 , 126.0, 125.9, 125.3, 81.2 , 74.6, 65.8, 52.4, 51.9, 38.6. Anal. Calcd for $\mathrm{C}_{24} \mathrm{H}_{22} \mathrm{O}_{5}$ : C, 73.83; H, 5.68. Found: C, 74.11; H, 5.91. 


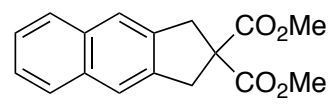

Dimethyl $1 H$-cyclopenta[b]naphthalene-2,2(3H)-dicarboxylate (6i).

Colorless amorphous (purified by preparative TLC, Hexane/EtOAc $=4 / 1$ ).

Yield: $24.7 \mathrm{mg}(79 \%)$.

IR (neat) 3054, 2979, 2955, 2902, 1742, 1434, 1306, 1259, 1198, 1178, 1164, 1052, 882 $\mathrm{cm}^{-1}$.

${ }^{1} \mathrm{H}$ NMR (300 MHz, $\left.\mathrm{CDCl}_{3}\right) \delta 7.74(\mathrm{dd}, 1 \mathrm{H}, J=6.6,63.3 \mathrm{~Hz}), 7.63(\mathrm{~s}, 2 \mathrm{H}), 7.39$ (dd, $1 \mathrm{H}, J=6.6,3.3 \mathrm{~Hz}), 3.74(\mathrm{~s}, 6 \mathrm{H}), 3.72(\mathrm{~s}, 4 \mathrm{H})$.

${ }^{13} \mathrm{C}$ NMR $\left(75 \mathrm{MHz} \mathrm{CDCl}_{3}\right) \delta 171.9,138.8,133.1,127.6,125.3,122.5,60.9,53.0,40.2$. Anal. Calcd for $\mathrm{C}_{17} \mathrm{H}_{16} \mathrm{O}_{4}$ : C, 71.82; H, 5.67. Found: C, 71.75; H, 5.43.

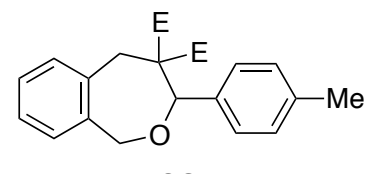

$\mathrm{E}=\mathrm{CO}_{2} \mathrm{Me}$

Dimethyl 3-(p-tolyl)-3,5-dihydrobenzo[c]oxepine-4,4(1H)-dicarboxylate (5j).

Colorless oil (purified by preparative TLC, Hexane/EtOAc $=4 / 1$ ).

Yield: $31.8 \mathrm{mg}(86 \%)$.

IR (neat) 3024, 2951, 2858, 1731, 1515, 1497, 1446, 1434, 1259, 1227, 1202, 1176, $1092,942 \mathrm{~cm}^{-1}$.

${ }^{1} \mathrm{H}$ NMR $\left(300 \mathrm{MHz}, \mathrm{CDCl}_{3}\right) \delta 7.32(\mathrm{~d}, 2 \mathrm{H}, J=8.1 \mathrm{~Hz}), 7.25-7.00(\mathrm{~m}, 6 \mathrm{H}), 5.39(\mathrm{~s}, 1 \mathrm{H})$, 5.25 (d, 1H, $J=15.3 \mathrm{~Hz}), 4.99$ (d, 1H, $J=15.3 \mathrm{~Hz}), 4.13$ (d, 1H, $J=14.1 \mathrm{~Hz}), 3.56$ (s, $3 \mathrm{H}), 3.24(\mathrm{~d}, 1 \mathrm{H}, J=14.1 \mathrm{~Hz}), 3.24$ (s, 3H), 2.31 (s, 3H).

${ }^{13} \mathrm{C}$ NMR $\left(75 \mathrm{MHz}, \mathrm{CDCl}_{3}\right) \delta 170.9,169.0,137.4,137.4,136.2,134.0,130.8,128.3$, $127.1,127.1,127.0,126.0,81.4,74.4,65.9,52.2,51.8,37.5,21.0$.

Anal. Calcd for $\mathrm{C}_{21} \mathrm{H}_{22} \mathrm{O}_{5}$ : C, 71.17; H, 6.26. Found: C, 71.35; H, 6.21.

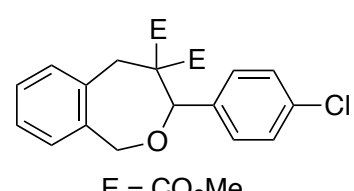

Dimethyl 3-(4-chlorophenyl)-3,5-dihydrobenzo[c]oxepine-4,4(1H)-dicarboxylate (5k).

Colorless oil (purified by preparative TLC, Hexane/EtOAc $=4 / 1$ ). 
Yield: $14.4 \mathrm{mg}(36 \%)$.

IR (neat) 3063, 3023, 3004, 2952, 2902, 2854, 1731, 1492, 1447, 1334, 1263, 1227 , $1203,1176,1091,1015,894 \mathrm{~cm}^{-1}$.

${ }^{1} \mathrm{H}$ NMR (300 MHz, $\left.\mathrm{CDCl}_{3}\right) \delta 7.39$ (d, 2H, $\left.J=8.4 \mathrm{~Hz}\right), 7.29-7.13(\mathrm{~m}, 5 \mathrm{H}), 7.06(\mathrm{~d}, 1 \mathrm{H}$, $J=8.4,8.4 \mathrm{~Hz}), 5.35(\mathrm{~s}, 1 \mathrm{H}), 5.28(\mathrm{~d}, 1 \mathrm{H}, J=15.0 \mathrm{~Hz}), 4.98(\mathrm{~d}, 1 \mathrm{H}, J=15.0 \mathrm{~Hz}), 4.10$ $(\mathrm{d}, 1 \mathrm{H}, J=14.1 \mathrm{~Hz}), 3.59(\mathrm{~s}, 3 \mathrm{H}), 3.25(\mathrm{~s}, 3 \mathrm{H}), 3.22(\mathrm{~d}, 1 \mathrm{H}, J=14.1 \mathrm{~Hz})$.

${ }^{13} \mathrm{C}$ NMR $\left(75 \mathrm{MHz}, \mathrm{CDCl}_{3}\right) \delta 170.7,168.8,137.8,137.1,134.1,133.5,130.7,128.6$, $127.8,127.4,127.3,126.1,80.6,74.7,65.9,52.4,51.9,37.7$.

Anal. Calcd for $\mathrm{C}_{20} \mathrm{H}_{19} \mathrm{ClO}_{5}$ : C, 64.09; H, 5.11. Found: C, 63.87; H, 5.30.

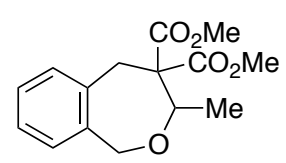

Dimethyl 3-methyl-3,5-dihydrobenzo[c]oxepine-4,4(1H)-dicarboxylate (5l).

Colorless oil (purified by preparative TLC, Hexane/EtOAc $=4 / 1$ ).

Yield: $11.7 \mathrm{mg}(36 \%)$.

IR (neat) 3022, 2979, 2952, 2904, 2846, 1731, 1496, 1454, 1379, 1268, 1257, 1239, 1204, 1107, 1090, 1041, $932 \mathrm{~cm}^{-1}$.

${ }^{1} \mathrm{H}$ NMR (300 MHz, $\left.\mathrm{CDCl}_{3}\right) \delta 7.20-7.02(\mathrm{~m}, 4 \mathrm{H}), 5.01(\mathrm{~d}, 1 \mathrm{H}, J=14.7 \mathrm{~Hz}), 4.78(\mathrm{~d}$, $1 \mathrm{H}, J=14.7 \mathrm{~Hz}), 4.31(\mathrm{q}, 1 \mathrm{H}, J=6.6 \mathrm{~Hz}), 3.69(\mathrm{~d}, 1 \mathrm{H}, J=14.4 \mathrm{~Hz}), 3.67(\mathrm{~s}, 3 \mathrm{H}), 3.65$ $(\mathrm{s}, 3 \mathrm{H}), 3.31(\mathrm{~d}, 1 \mathrm{H}, J=14.4 \mathrm{~Hz}), 1.37(\mathrm{~d}, 3 \mathrm{H}, J=6.6 \mathrm{~Hz})$.

${ }^{13} \mathrm{C} \mathrm{NMR}\left(75 \mathrm{MHz}, \mathrm{CDCl}_{3}\right) \delta 170.6,169.2,138.2,135.3,130.8,127.4,127.0,126.8$, $78.4,73.6,62.3,52.3,51.9,38.7,18.6$.

Anal. Calcd for $\mathrm{C}_{15} \mathrm{H}_{18} \mathrm{O}_{5}$ : C, 64.74; H, 6.52. Found: C, 64.97; H, 6.64. 


\section{References}

1) Dai, Y.; Feng, X.; Liu, H,; Jiang, H.; Bao, M. J. Org. Chem. 2011, 76, 10068.

2) Dreier, D.; Resetar, M.; Temml, V.; Rycek, L.; Kratena, N.; Scnurch, M.; Schuster, D.; Dirsch, V. M.; Mihovilovic, M. D. Org. Biomol. Chem. 2018, 16, 7019.

3) Musso, D. L.; Orr, G. F.; Cochran, F. R.; Kelley, J. L.; Selph, J. L.; Rigdon, G. C.; Cooper, B. R.; Jones, M. L. J. Med. Chem. 2003, 46, 409.

4) Watanabe, K.; Mino, T.; Hatta, C.; Ito, S.; Sakamoto, M. Org. Biomol. Chem. 2015, $13,11645$.

5) Rousseaux, S.; Garcia-Fortanet, J.; Sanchez, M. A. D. A.; Buchwald, S. L. J. Am. Chem. Soc. 2011, 133, 9282.

6) Clive, D. L. J.; Fletcher, S. P.; Liu, D. J. Org. Chem. 2004, 69, 3282. 
${ }^{1} \mathrm{H}$ NMR spectrum of $\mathbf{s} 2\left(\mathrm{CDCl}_{3}, 300 \mathrm{MHz}\right)$.

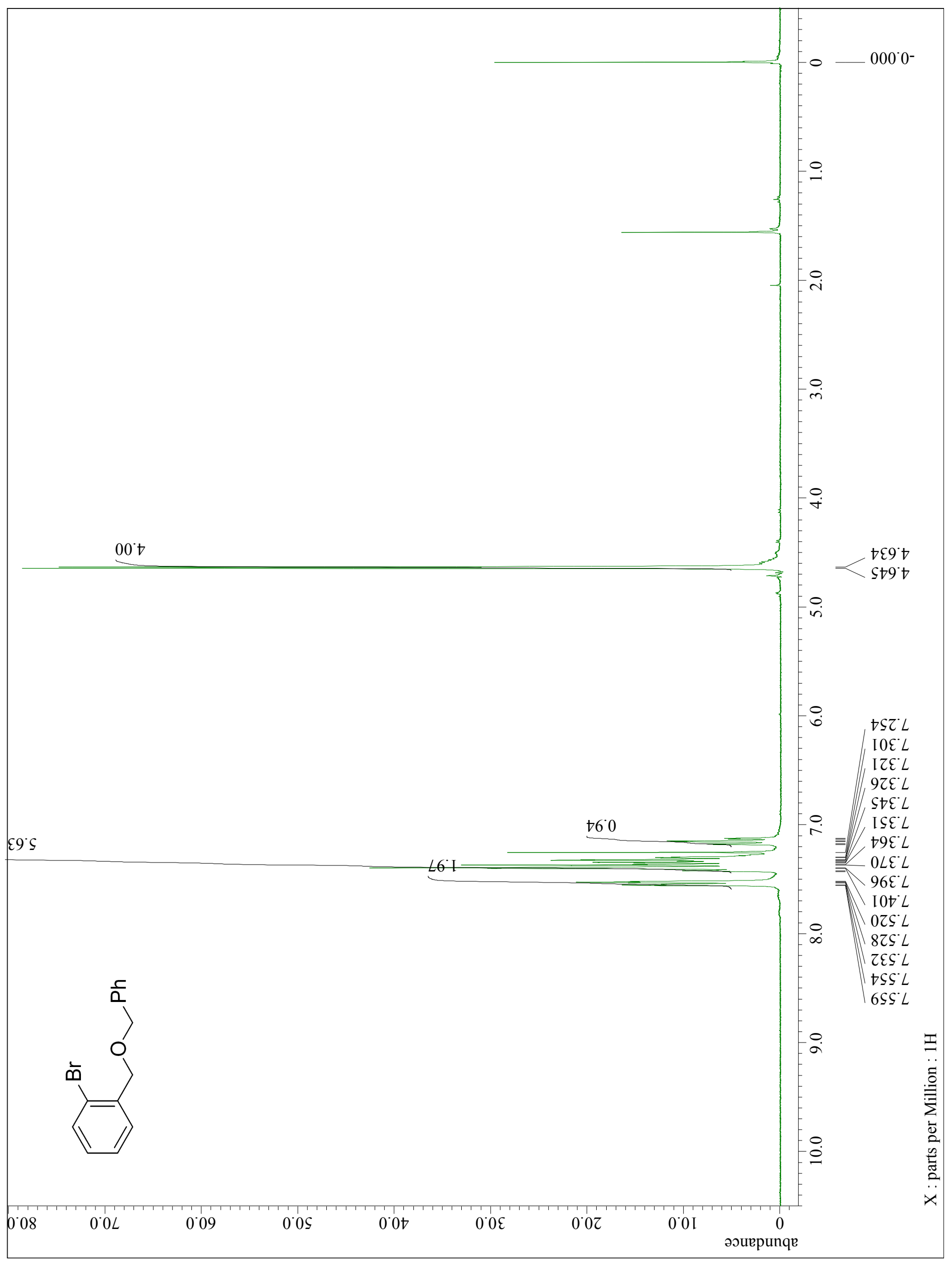


${ }^{13} \mathrm{C} \mathrm{NMR}$ spectrum of $\mathbf{s} 2\left(\mathrm{CDCl}_{3}, 75 \mathrm{MHz}\right)$.

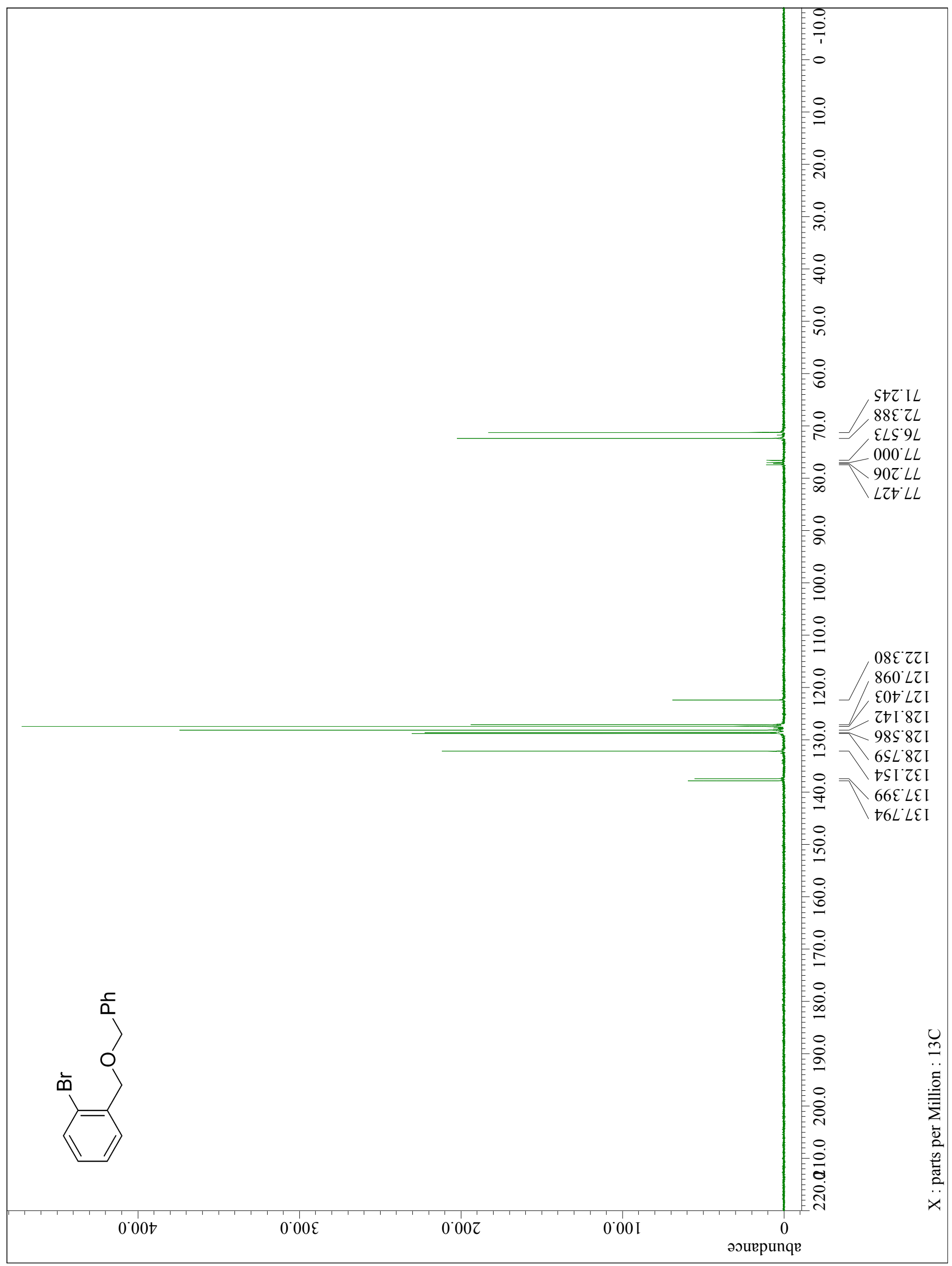


${ }^{1} \mathrm{H}$ NMR spectrum of $\mathbf{s} 3\left(\mathrm{CDCl}_{3}, 300 \mathrm{MHz}\right)$.

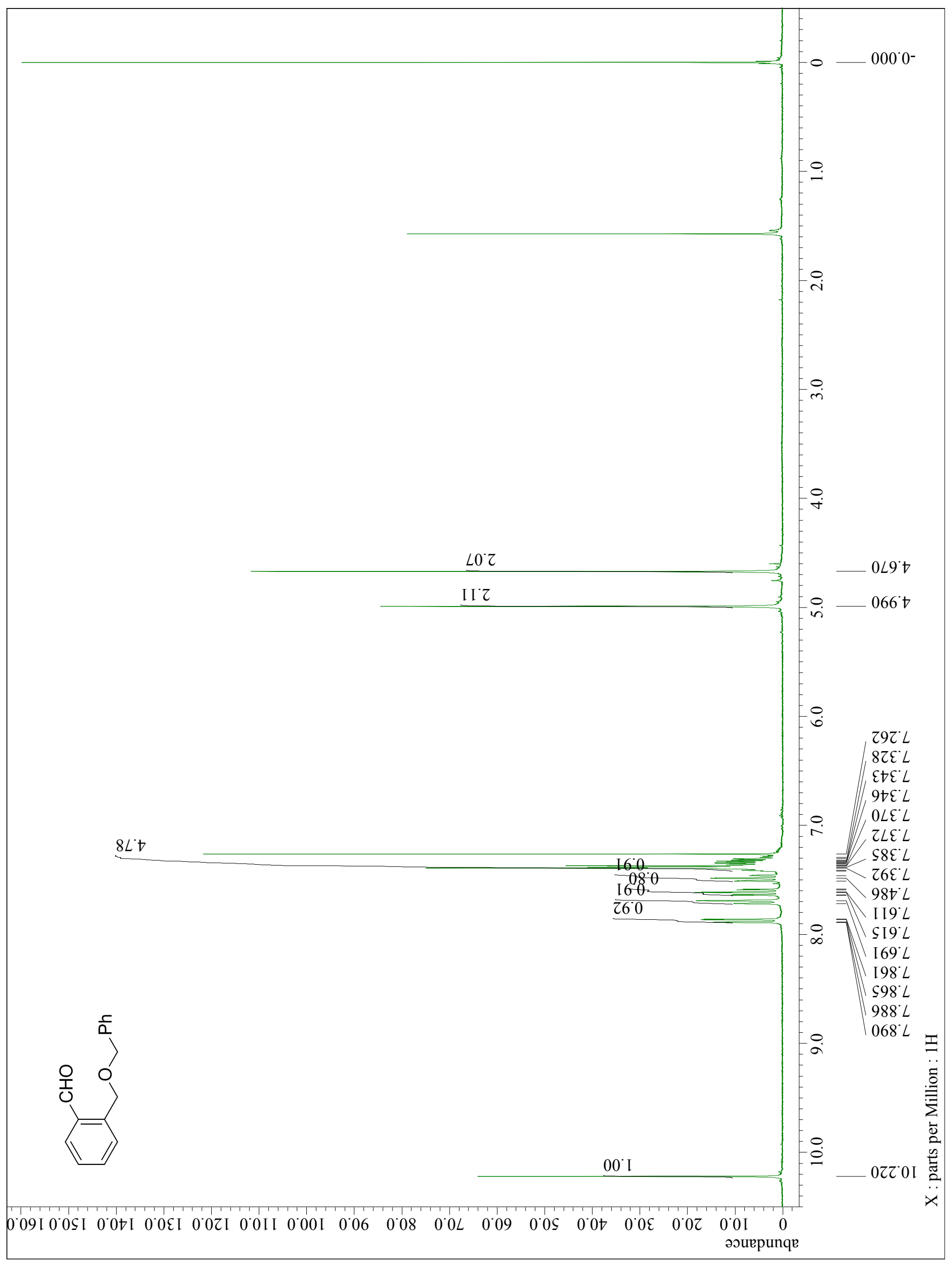


${ }^{13} \mathrm{C} \mathrm{NMR}$ spectrum of $\mathbf{s} 3\left(\mathrm{CDCl}_{3}, 75 \mathrm{MHz}\right)$.

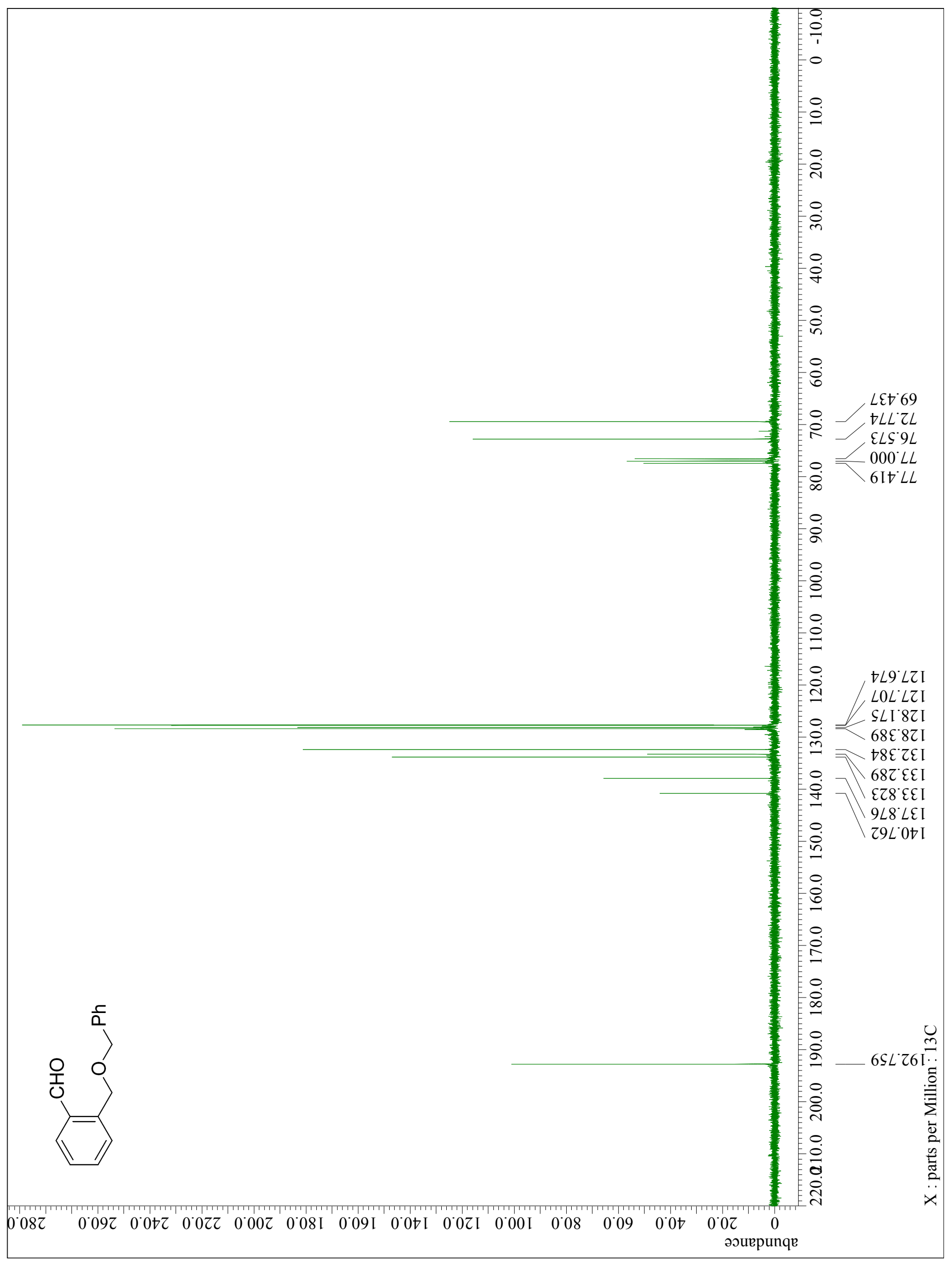


${ }^{1} \mathrm{H}$ NMR spectrum of $\mathbf{3 a}\left(\mathrm{CDCl}_{3}, 300 \mathrm{MHz}\right)$.

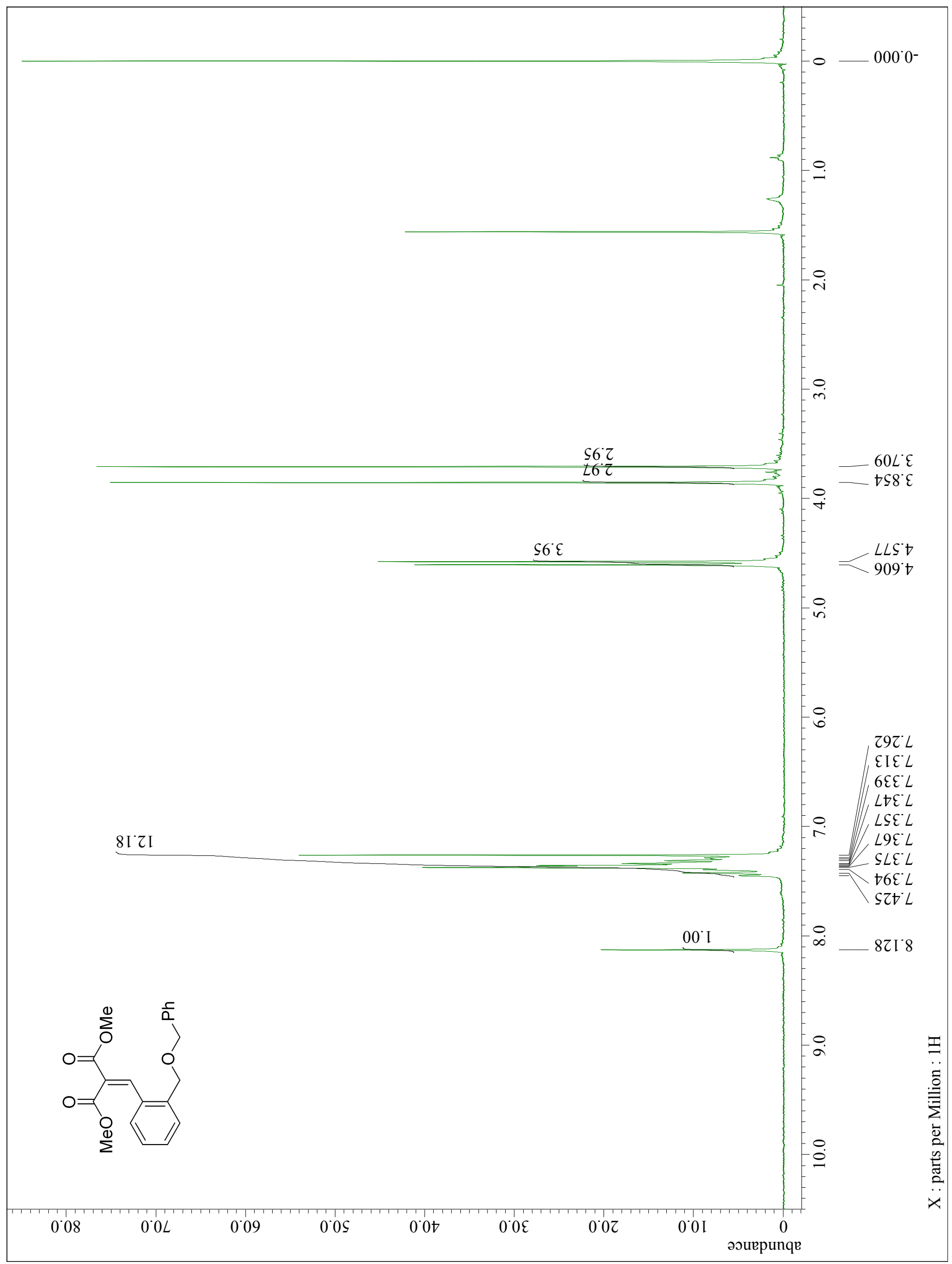




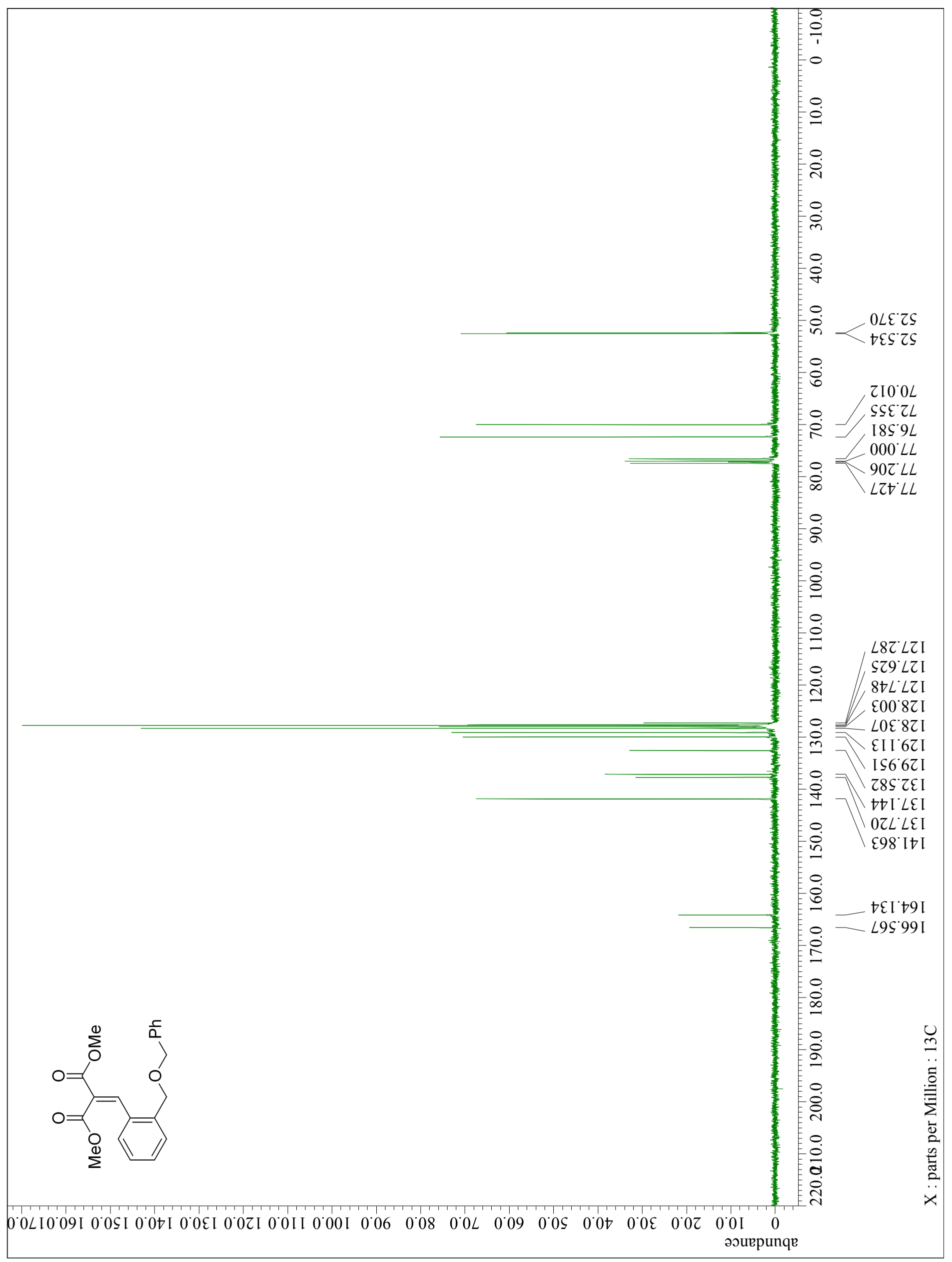


${ }^{1} \mathrm{H}$ NMR spectrum of $\mathbf{s} 4\left(\mathrm{CDCl}_{3}, 300 \mathrm{MHz}\right)$.

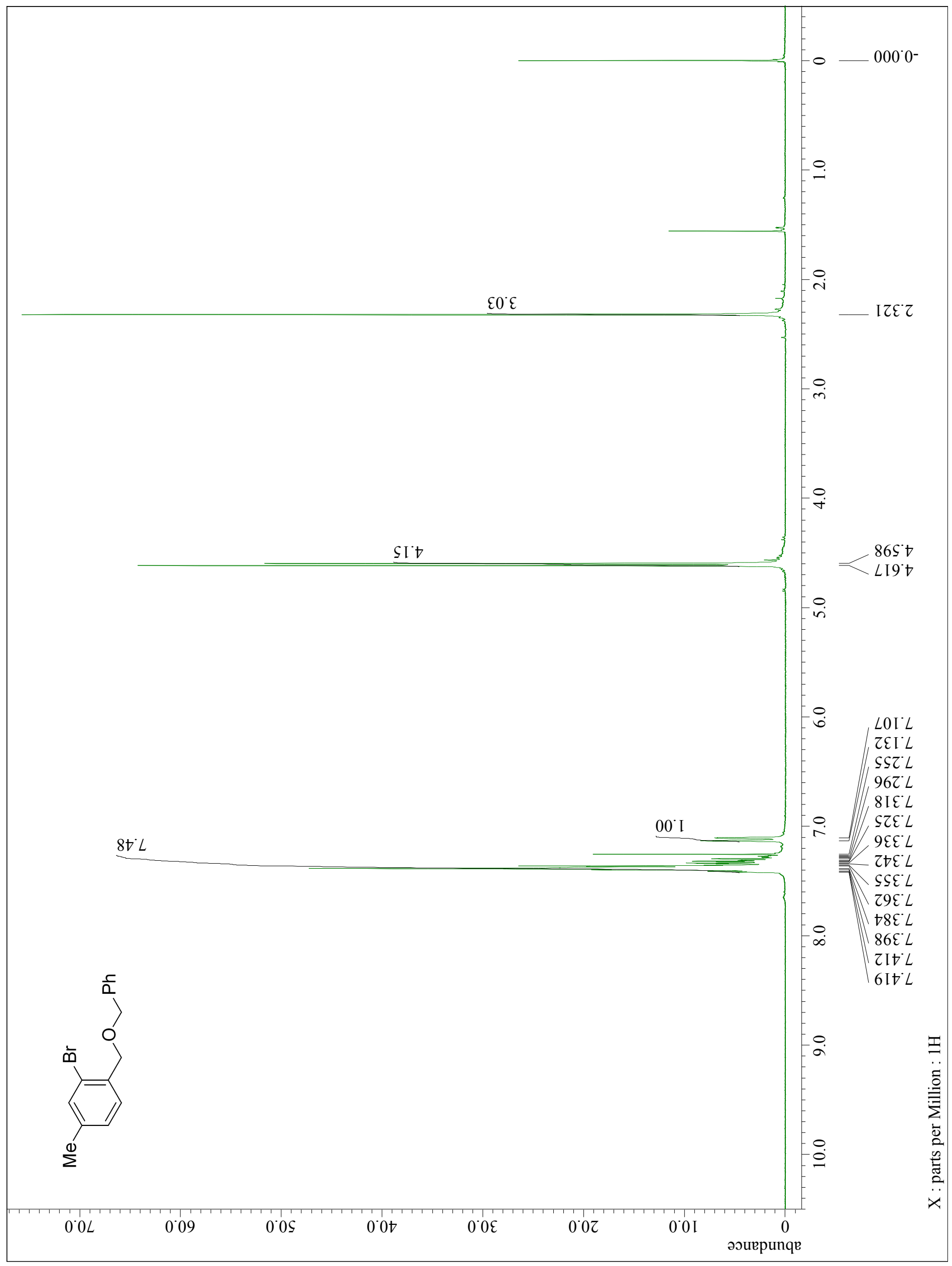




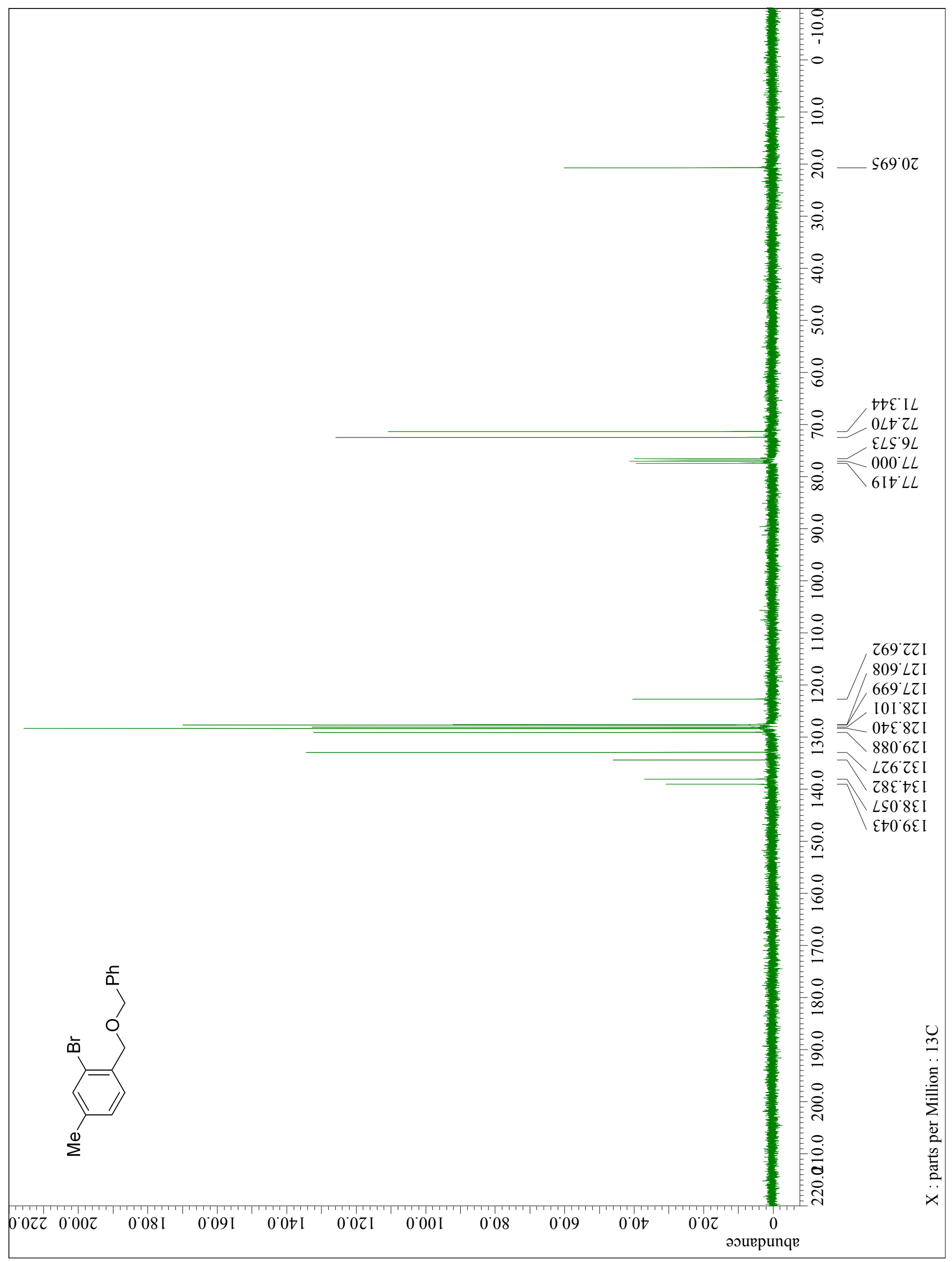


${ }^{1} \mathrm{H}$ NMR spectrum of $\mathbf{s 5}\left(\mathrm{CDCl}_{3}, 300 \mathrm{MHz}\right)$.

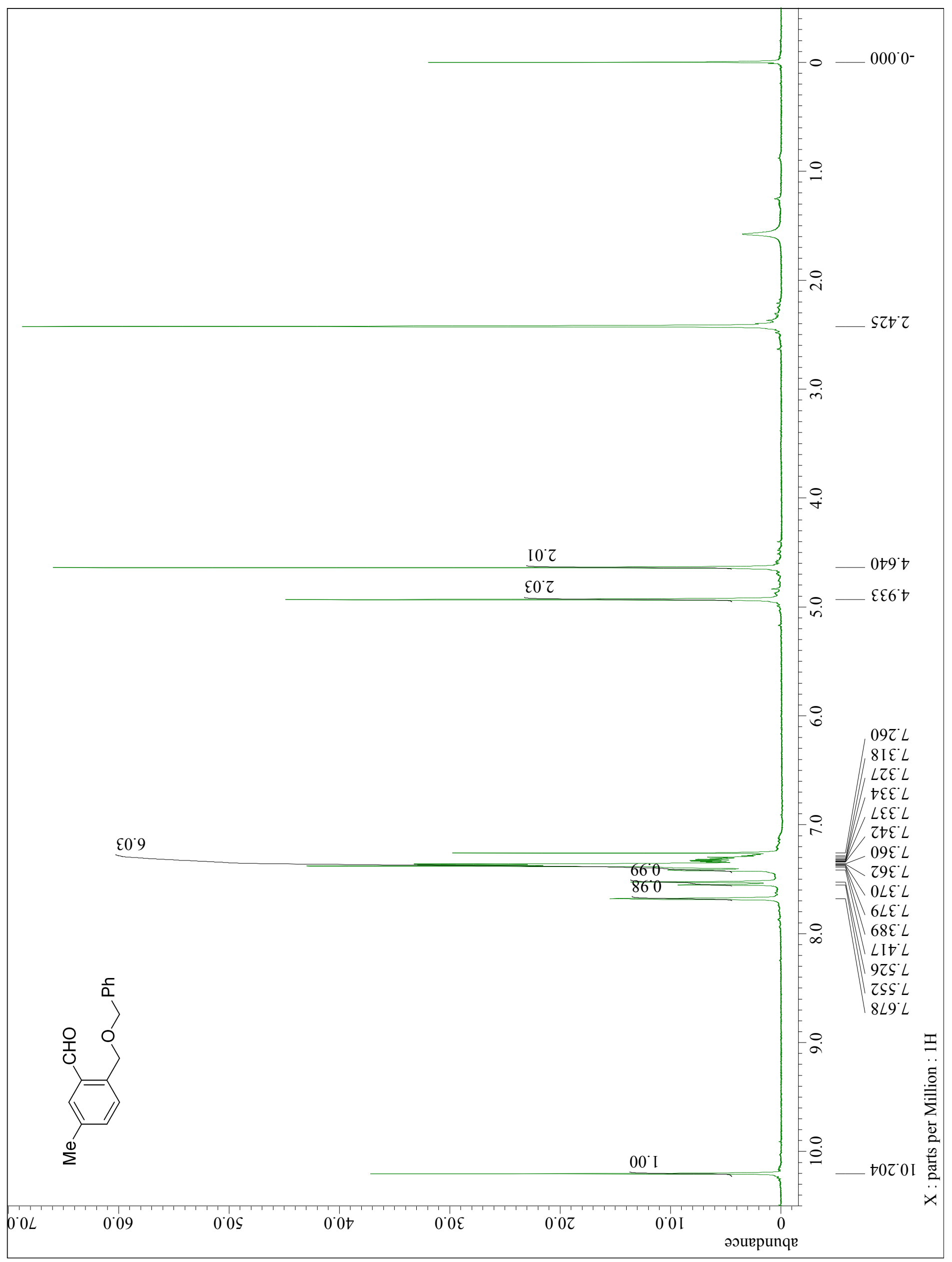


${ }^{13} \mathrm{C} \mathrm{NMR}$ spectrum of $\mathbf{s 5}\left(\mathrm{CDCl}_{3}, 75 \mathrm{MHz}\right)$.

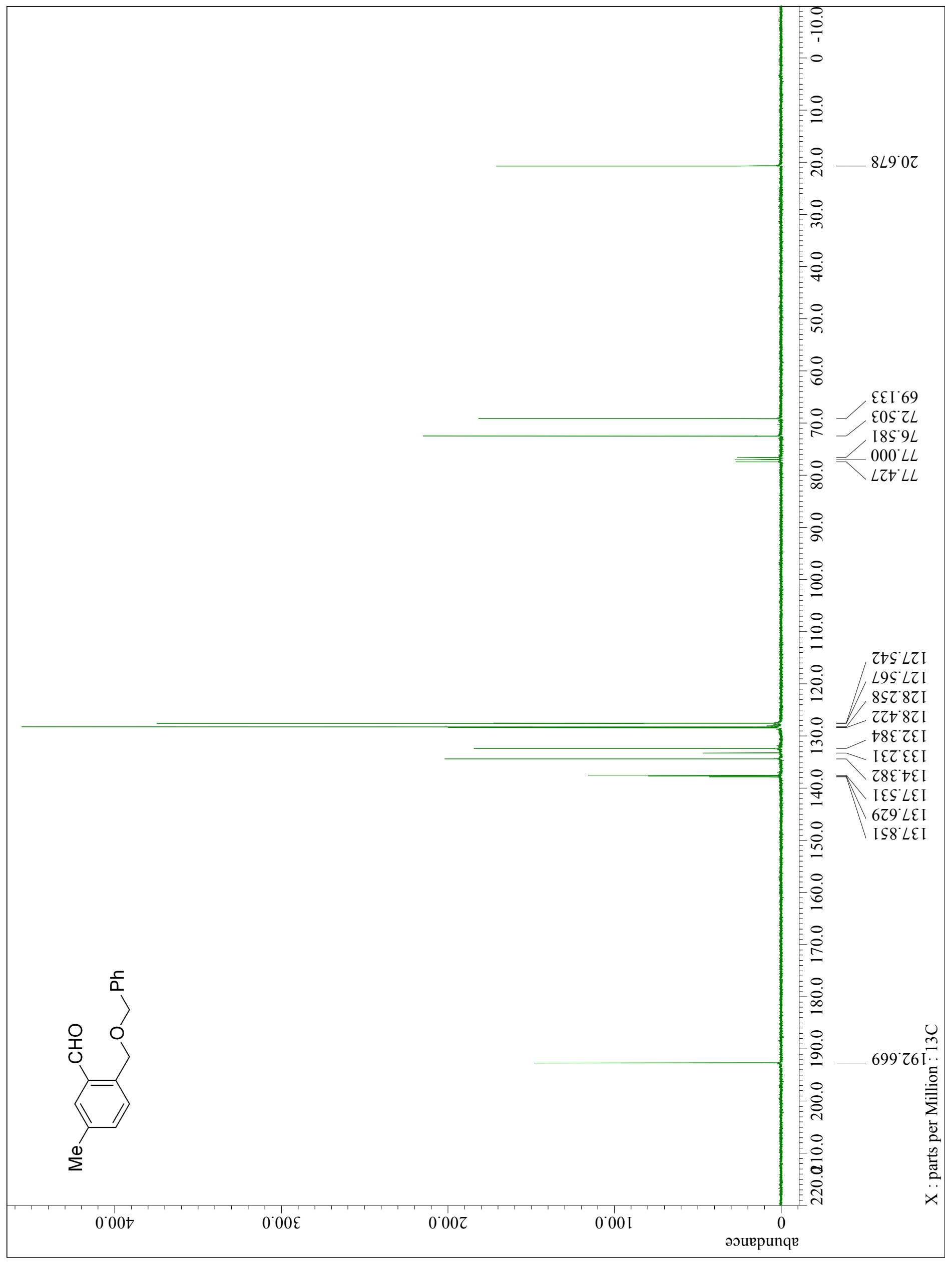


${ }^{1} \mathrm{H}$ NMR spectrum of $\mathbf{3 b}\left(\mathrm{CDCl}_{3}, 300 \mathrm{MHz}\right)$.

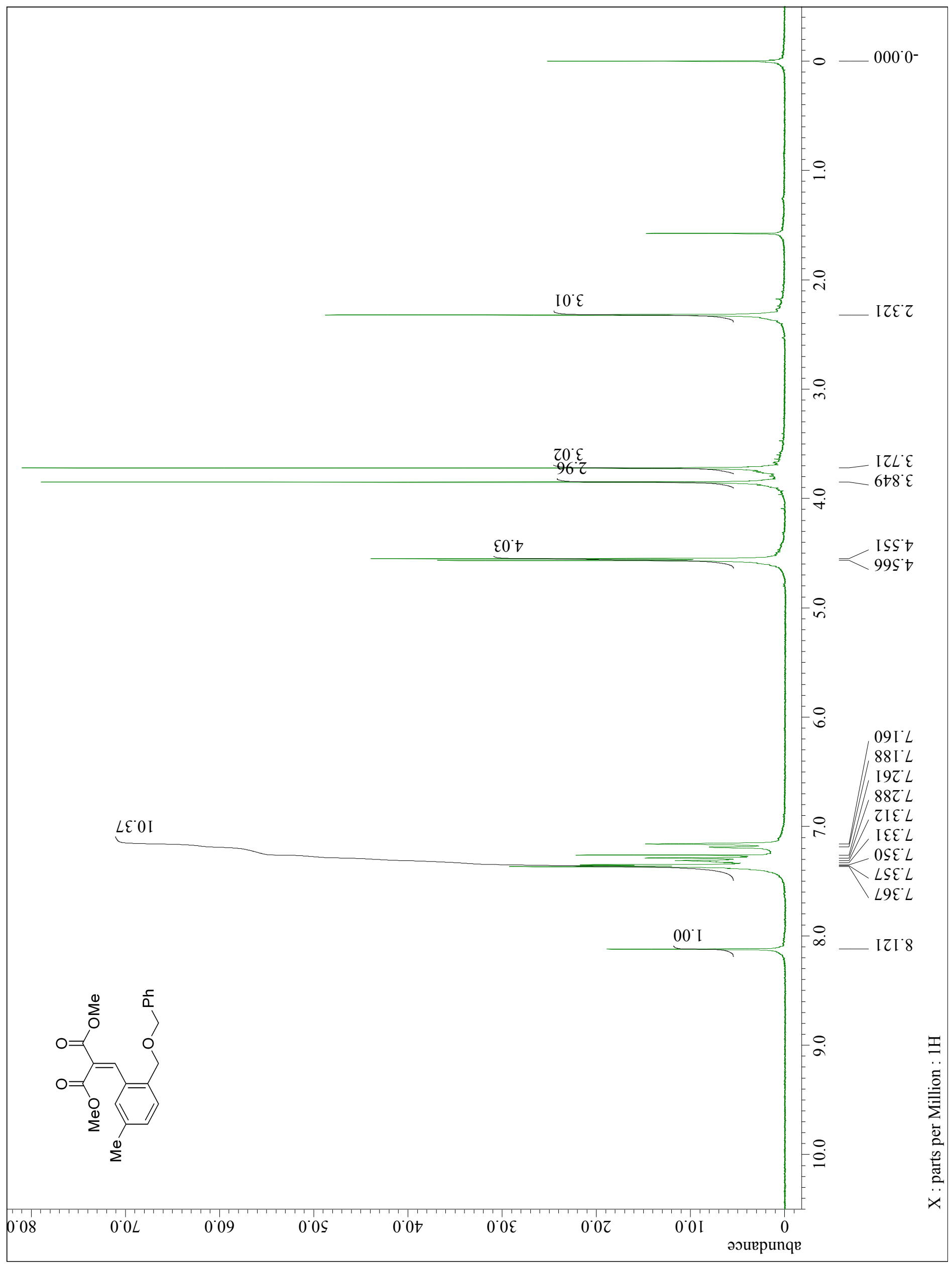




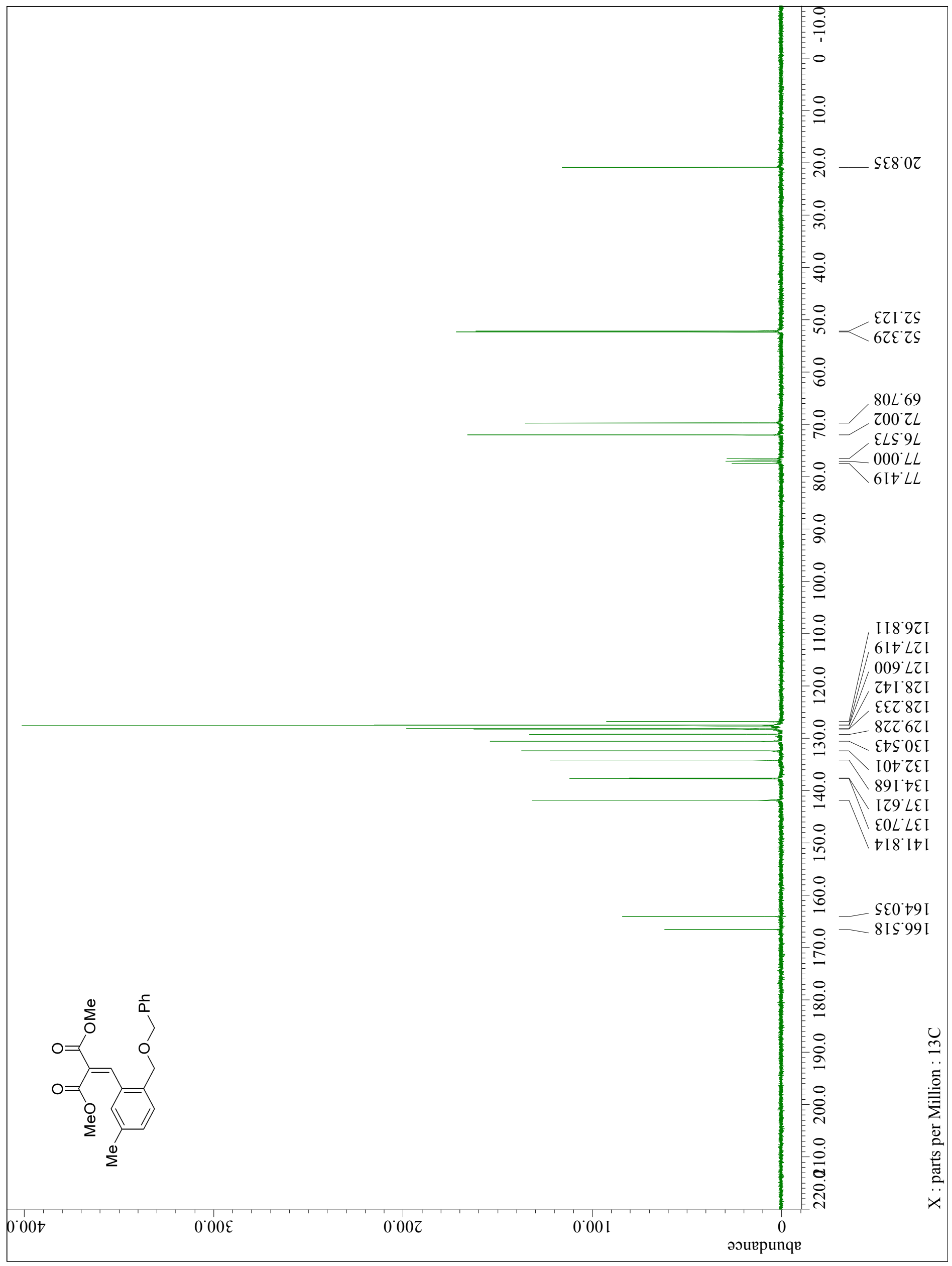




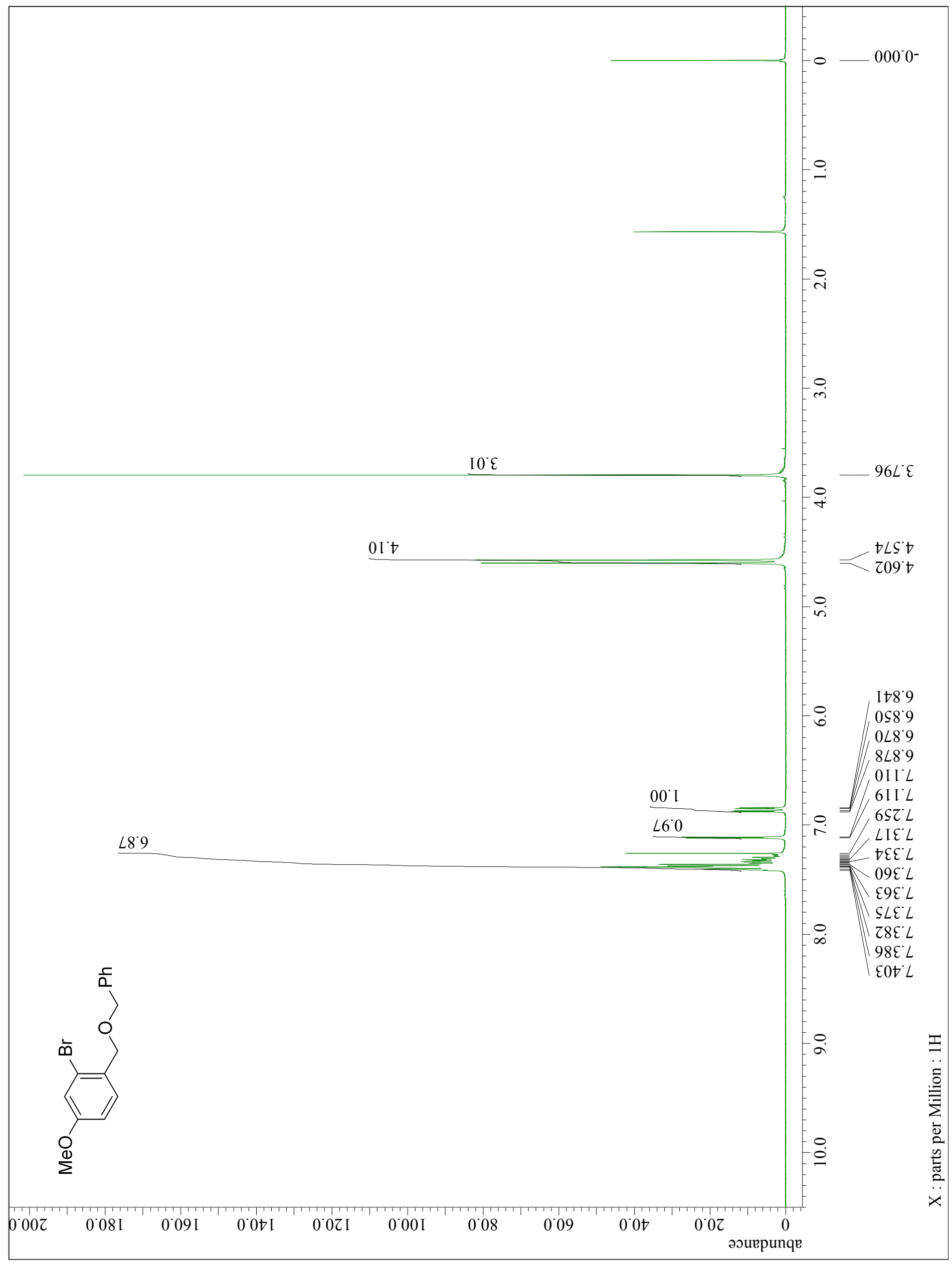




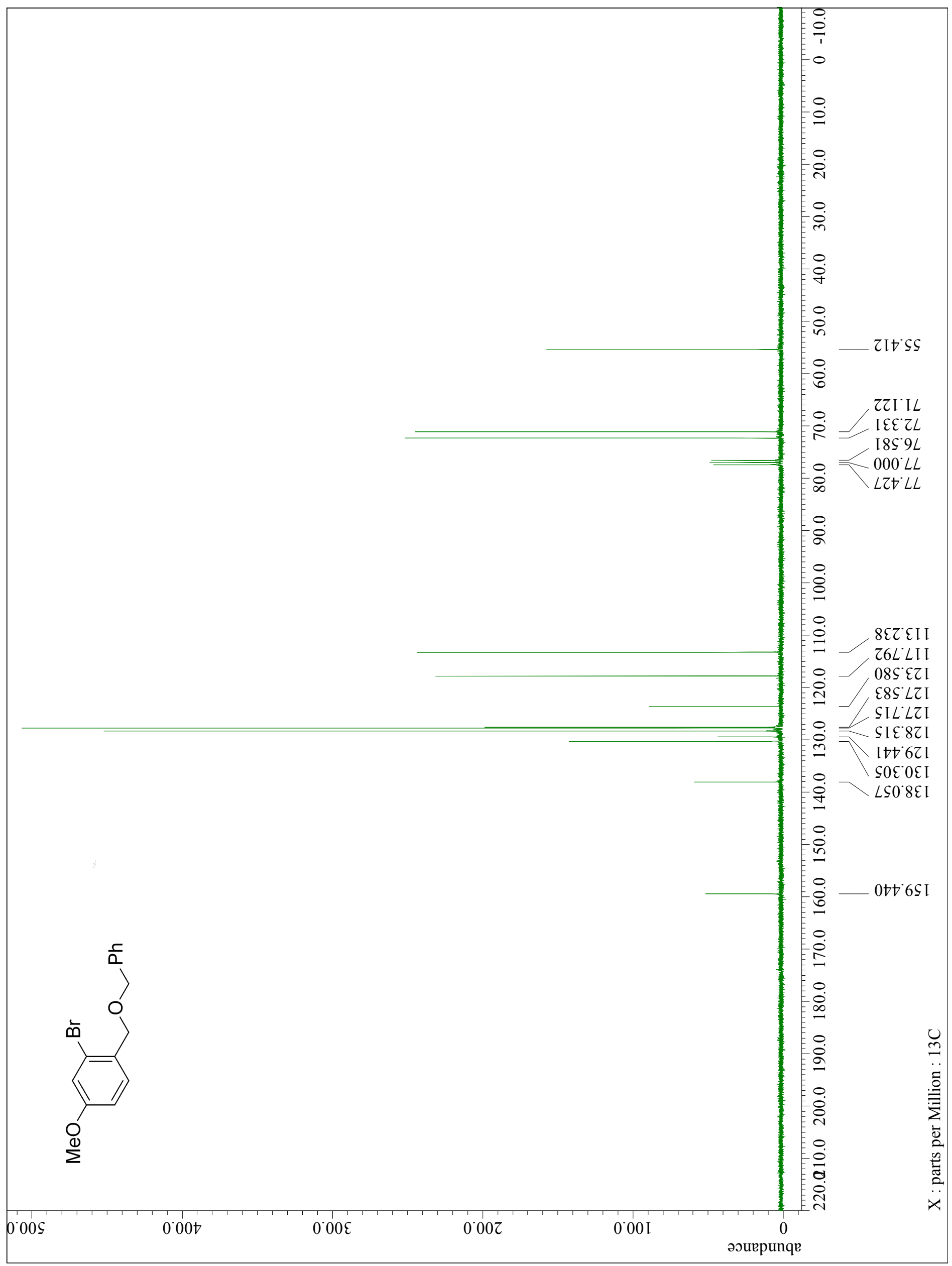


${ }^{1} \mathrm{H}$ NMR spectrum of $\mathbf{s} 7\left(\mathrm{CDCl}_{3}, 300 \mathrm{MHz}\right)$.

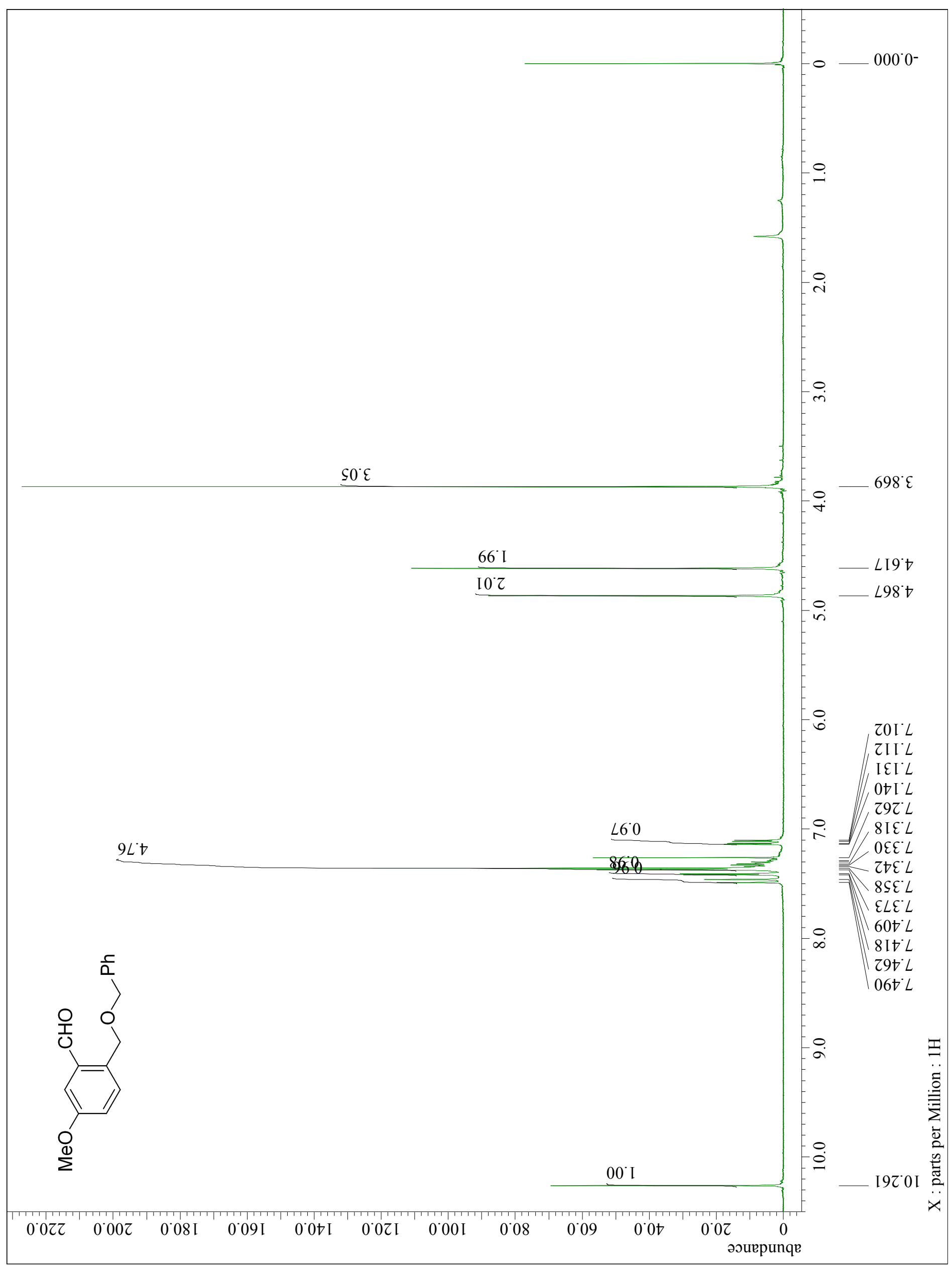


${ }^{13} \mathrm{C}$ NMR spectrum of $\mathbf{s} 7\left(\mathrm{CDCl}_{3}, 75 \mathrm{MHz}\right)$.

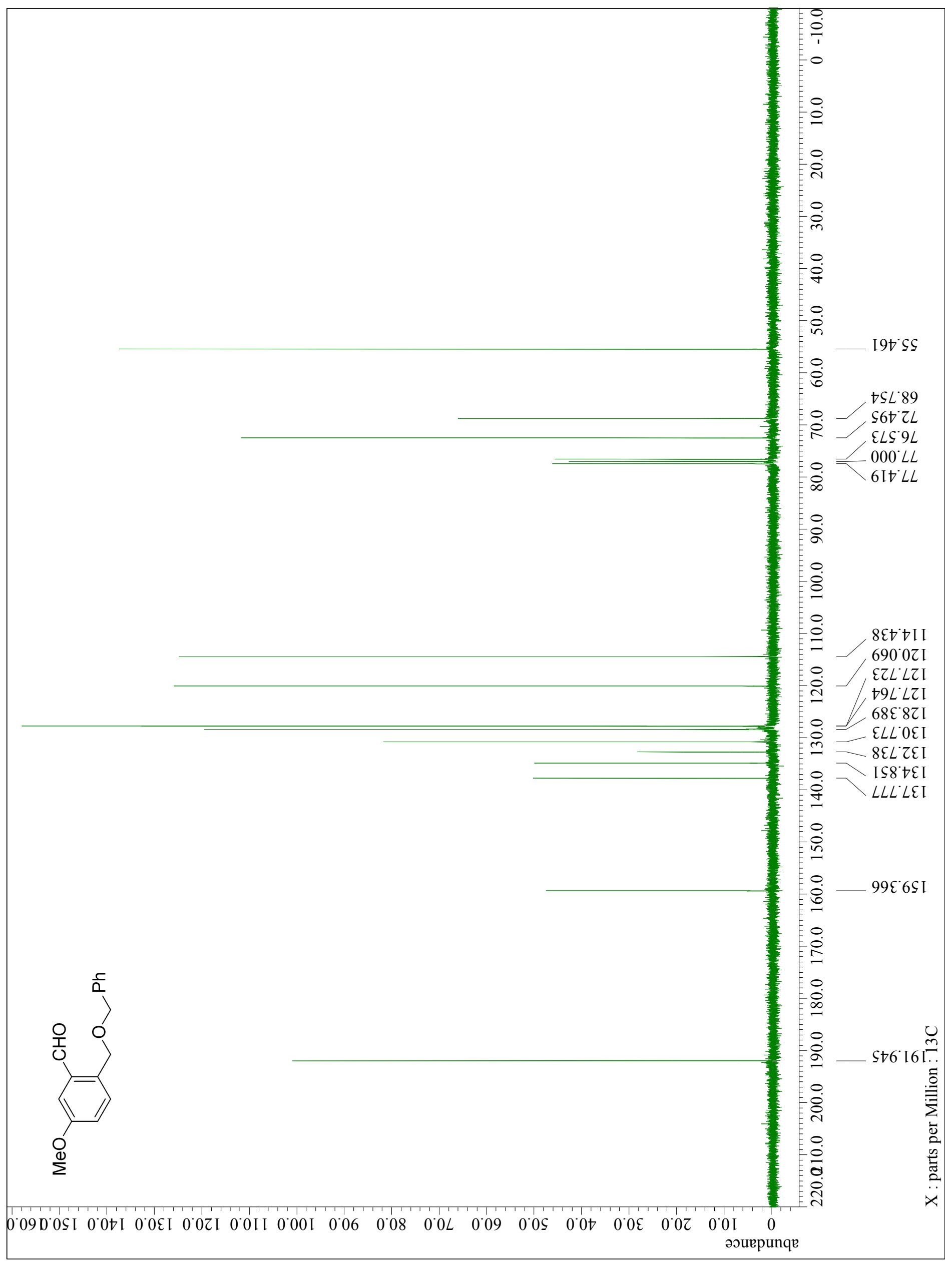


${ }^{1} \mathrm{H}$ NMR spectrum of $\mathbf{3 c}\left(\mathrm{CDCl}_{3}, 300 \mathrm{MHz}\right)$.

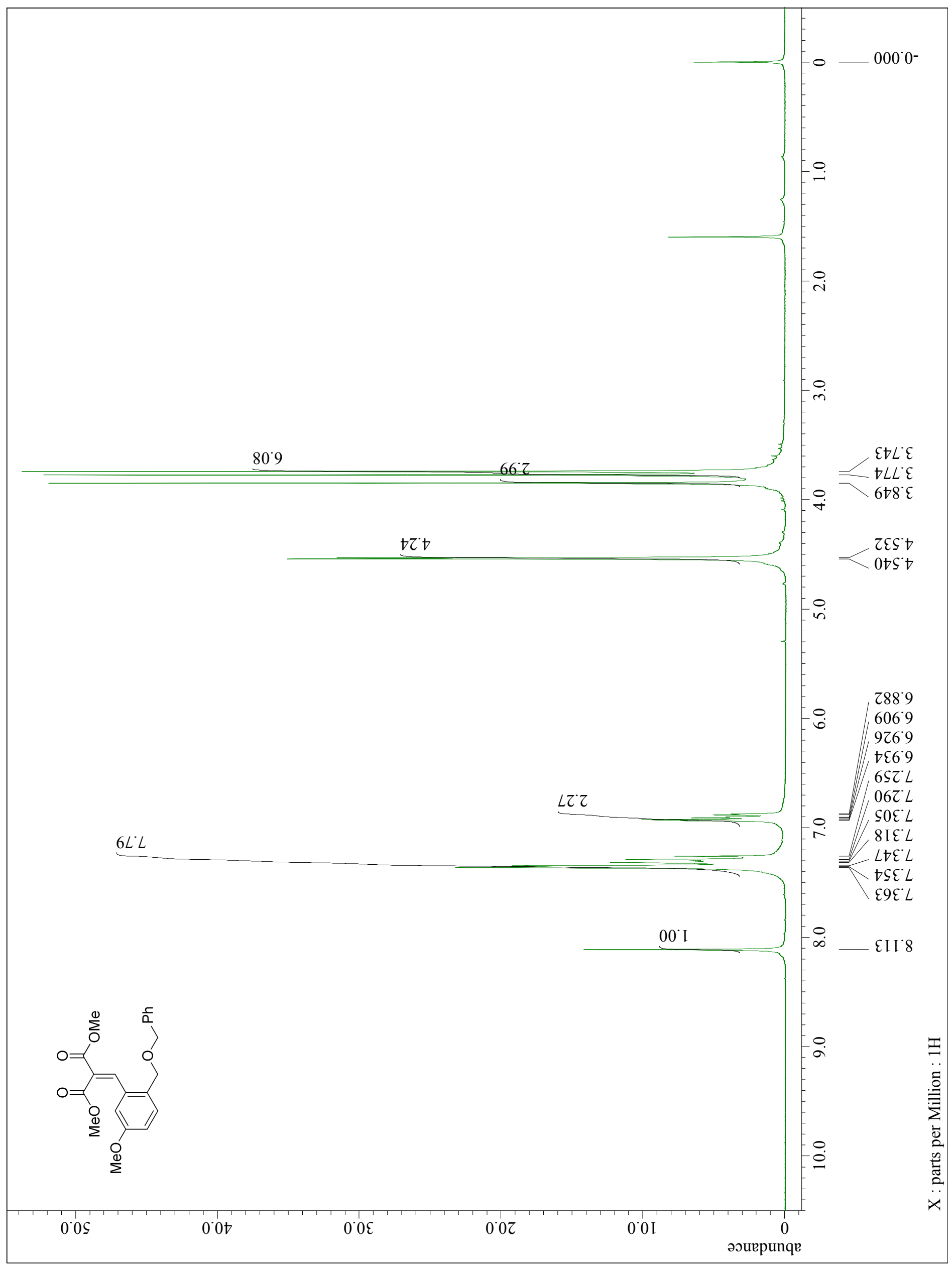




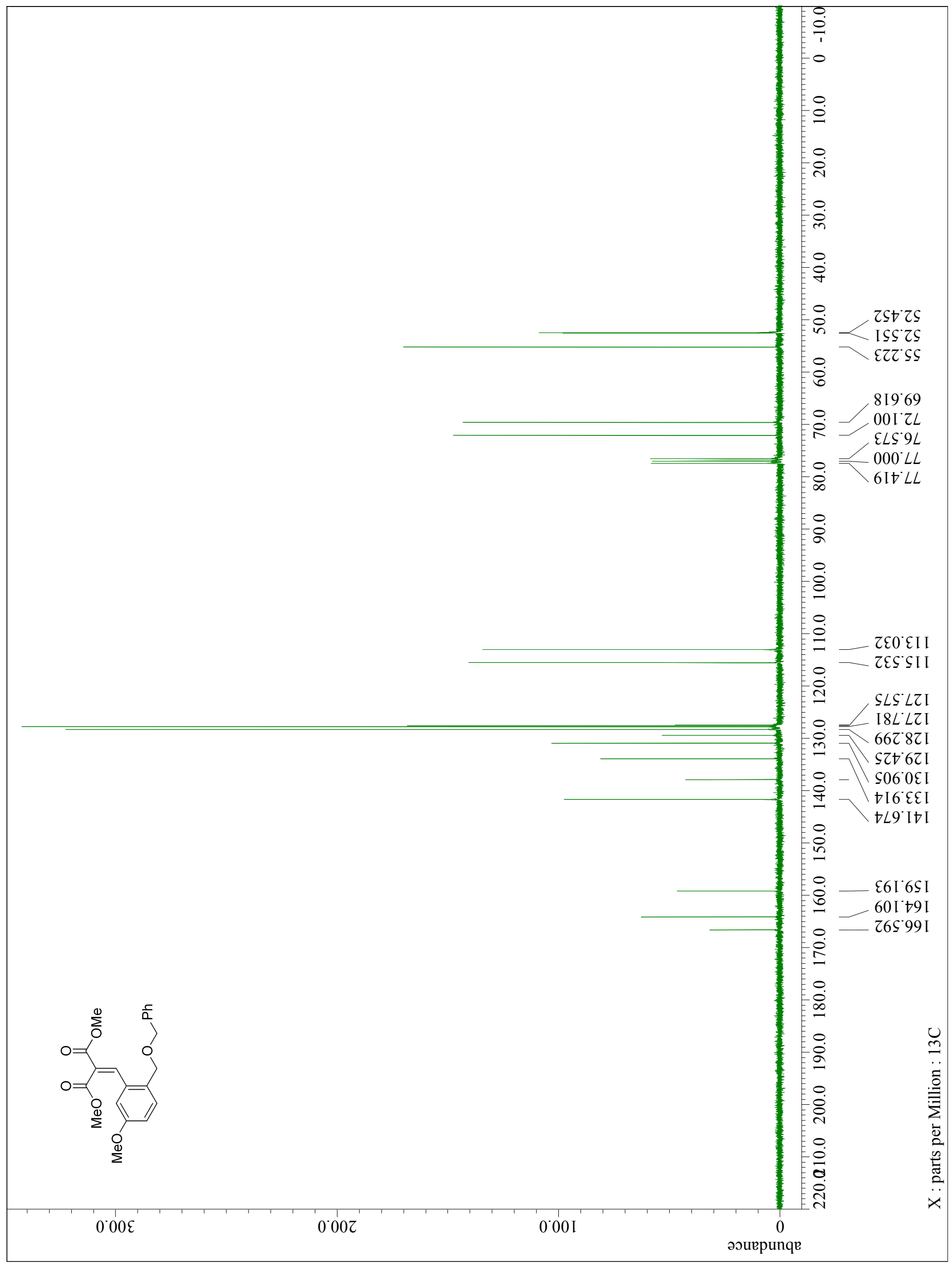


${ }^{1} \mathrm{H}$ NMR spectrum of $\mathbf{s 8}\left(\mathrm{CDCl}_{3}, 300 \mathrm{MHz}\right)$.

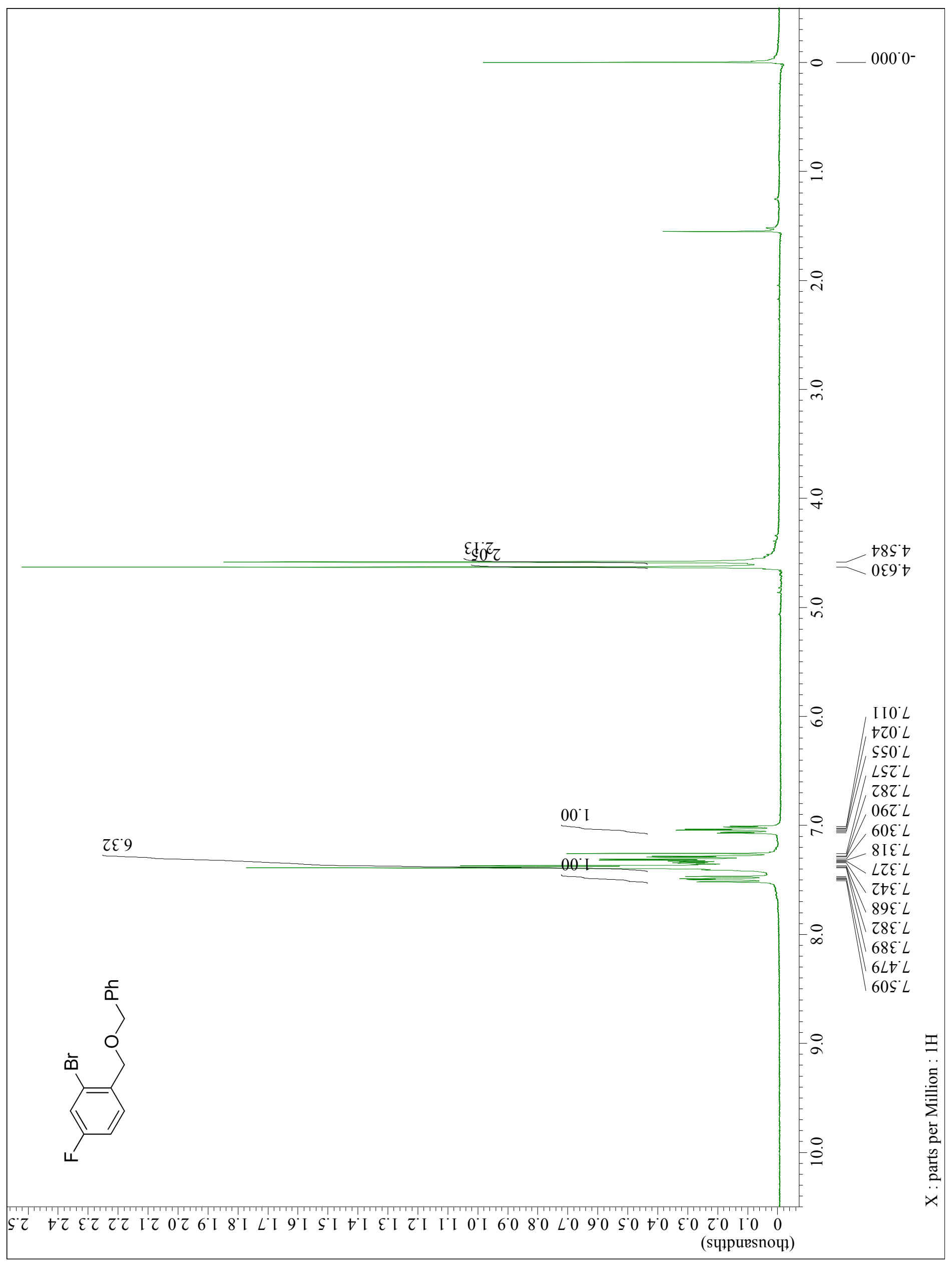




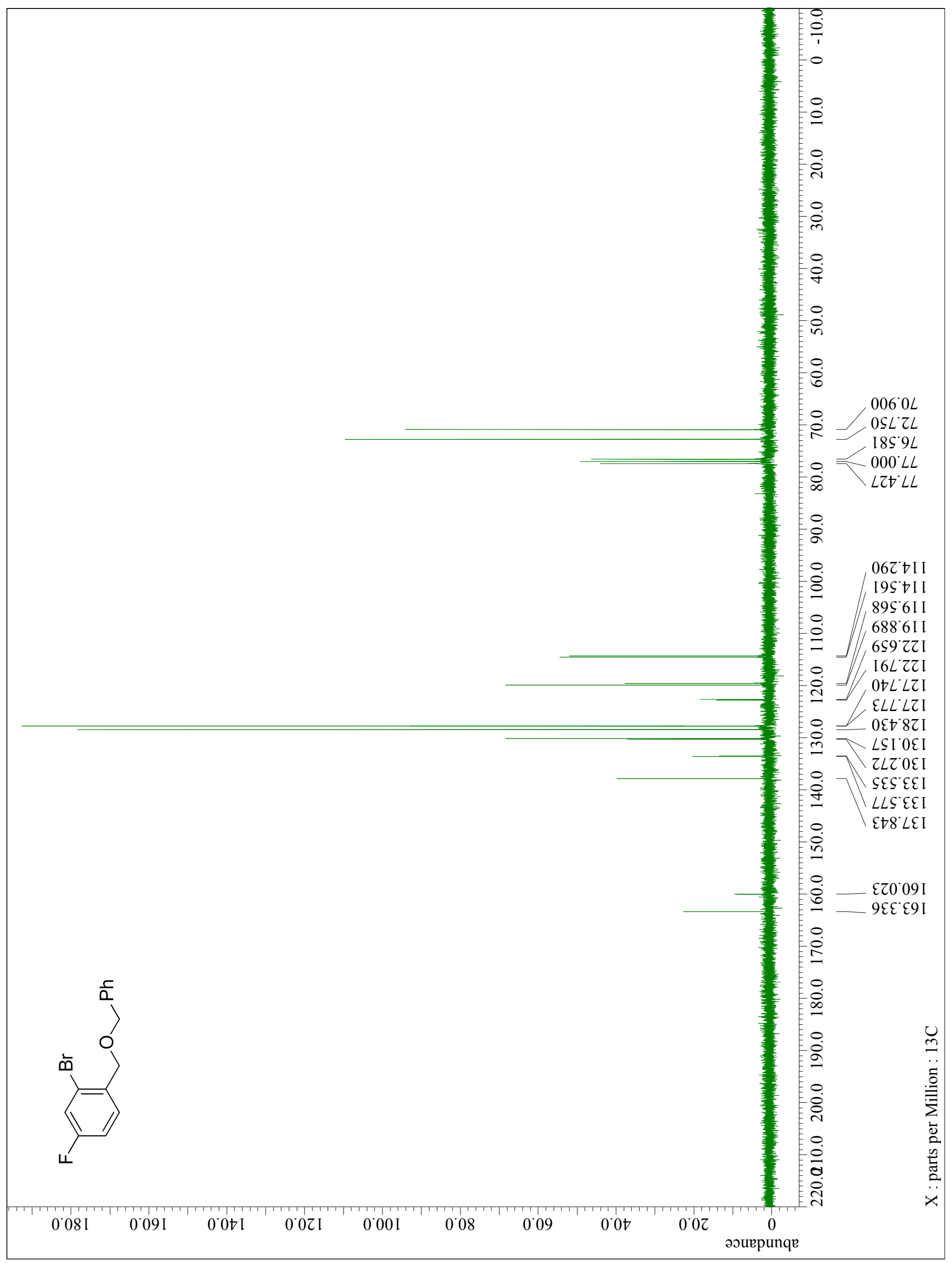


${ }^{19} \mathrm{~F}$ NMR spectrum of $\mathbf{s 8}\left(\mathrm{CDCl}_{3}, 283 \mathrm{MHz}\right)$.

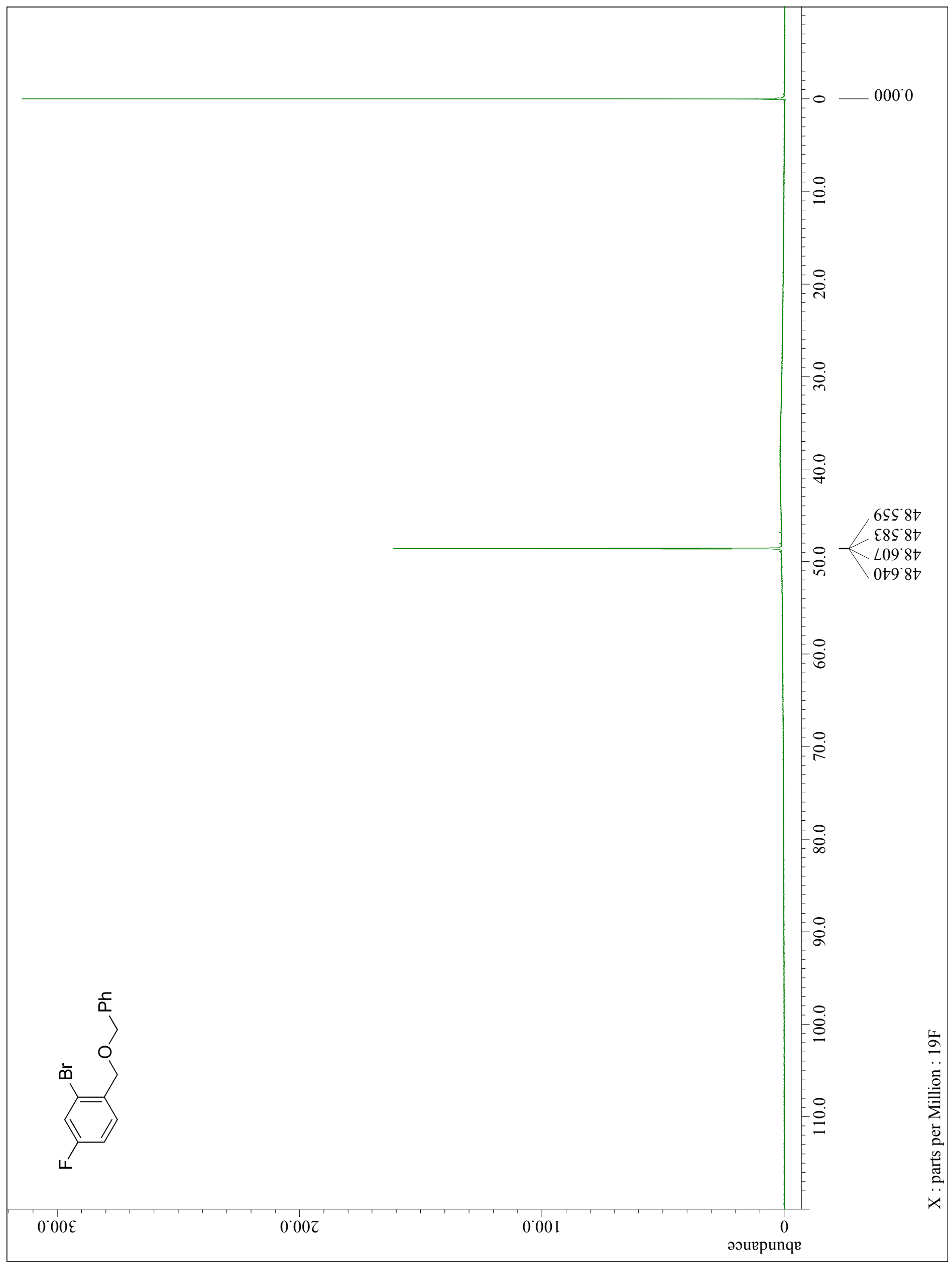




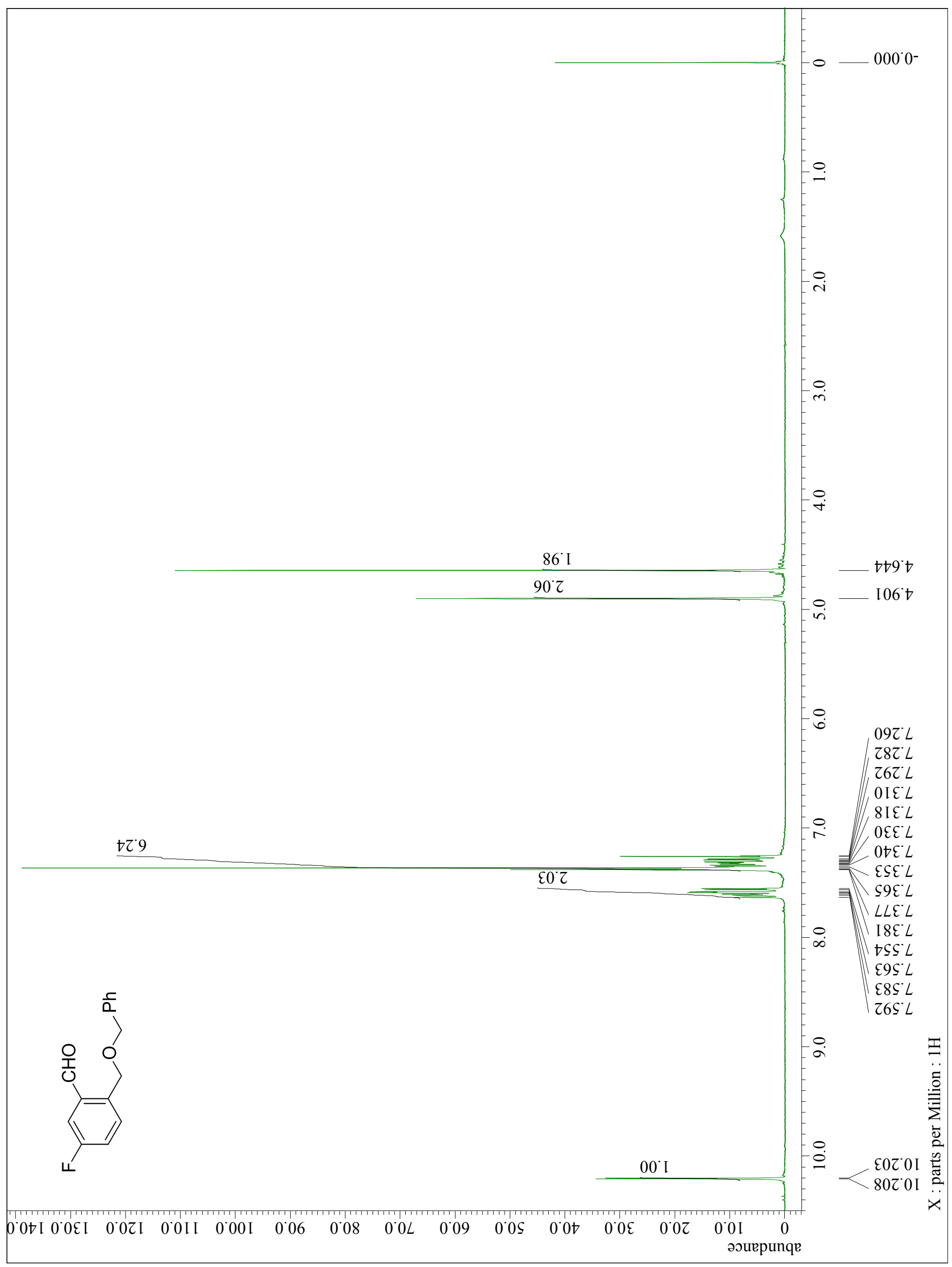


${ }^{13} \mathrm{C} \mathrm{NMR}$ spectrum of $\mathbf{s} 9\left(\mathrm{CDCl}_{3}, 75 \mathrm{MHz}\right)$.

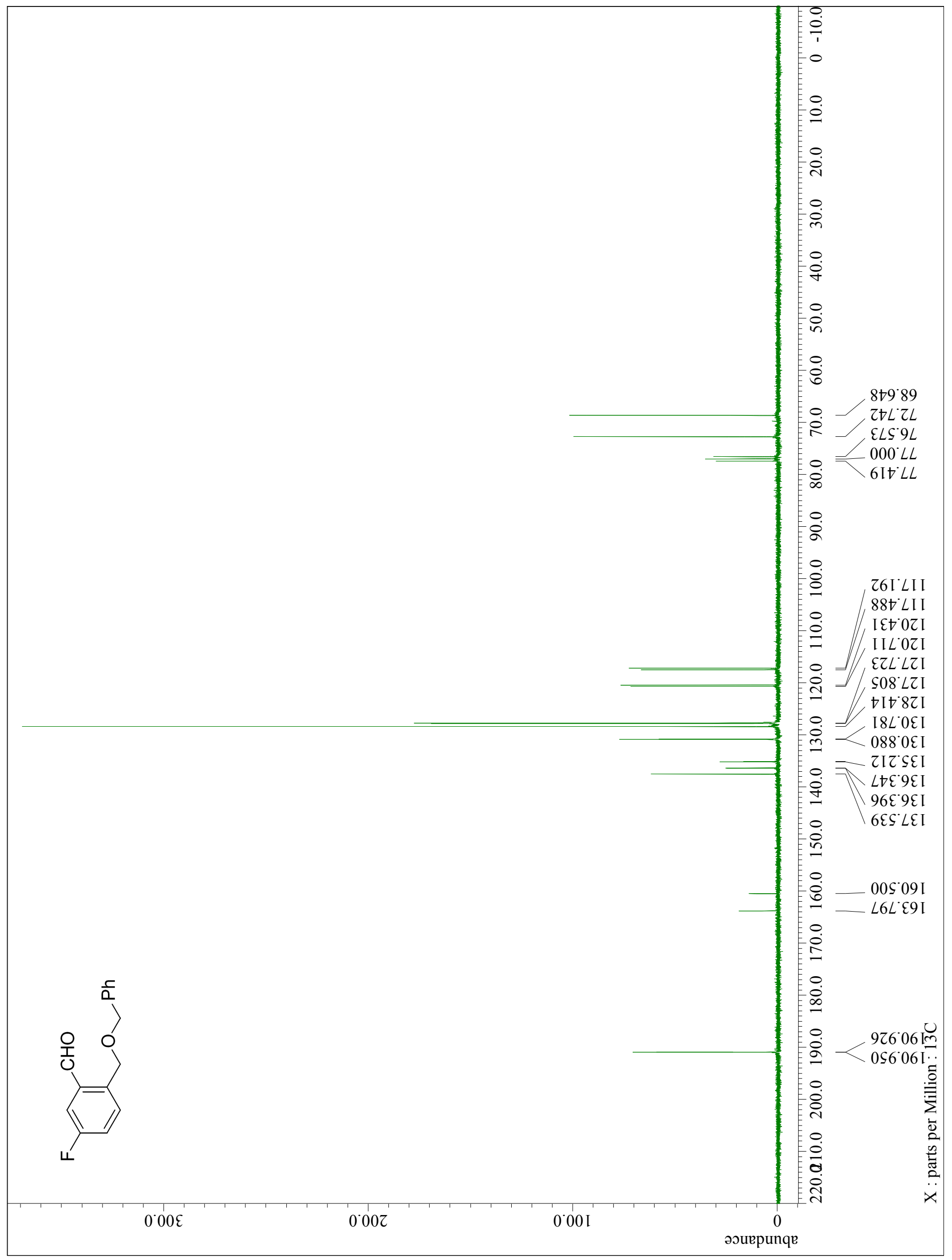


${ }^{19} \mathrm{~F}$ NMR spectrum of $\mathbf{s} 9\left(\mathrm{CDCl}_{3}, 283 \mathrm{MHz}\right)$.

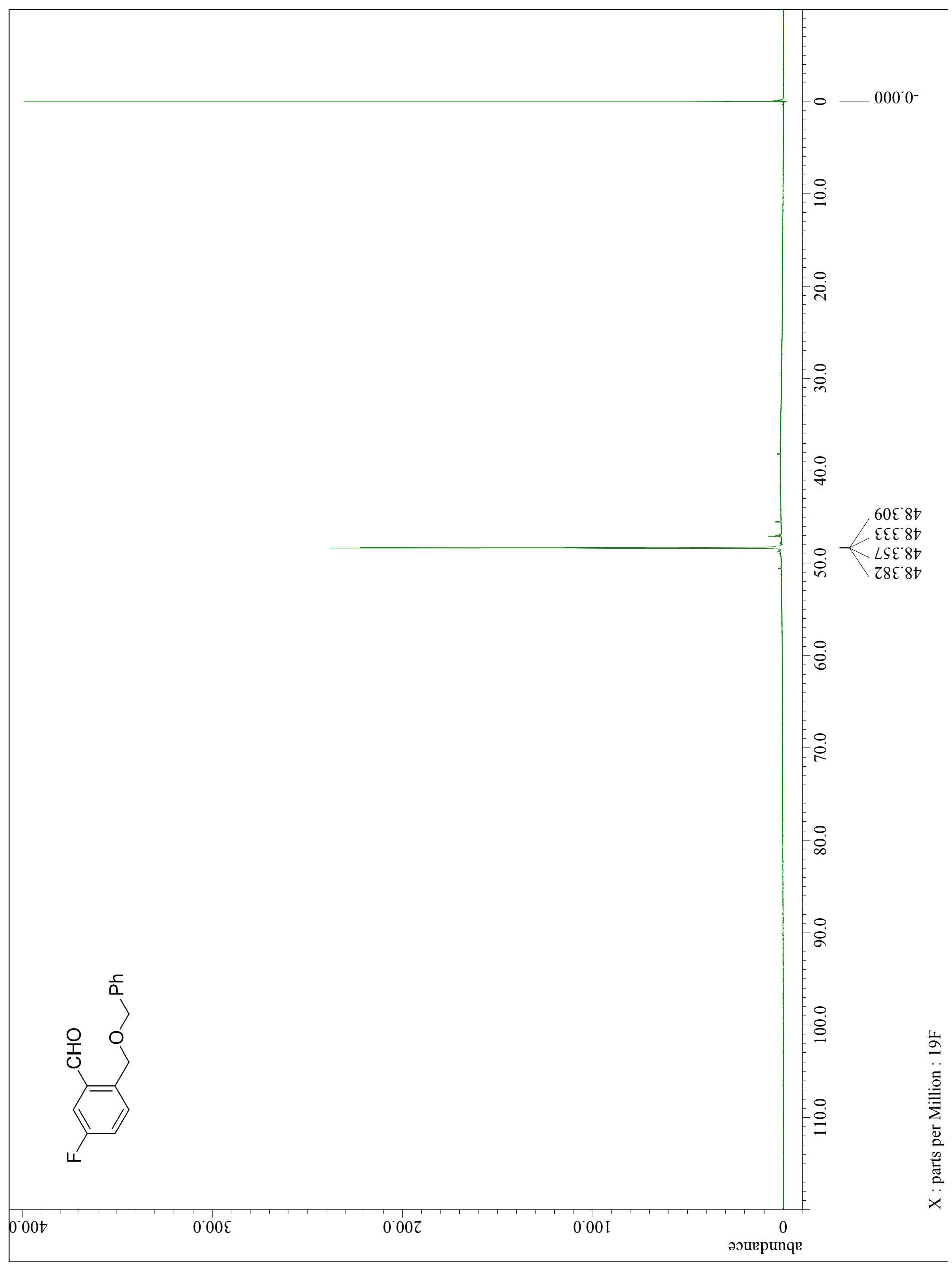


${ }^{1} \mathrm{H}$ NMR spectrum of $\mathbf{3 d}\left(\mathrm{CDCl}_{3}, 300 \mathrm{MHz}\right)$.

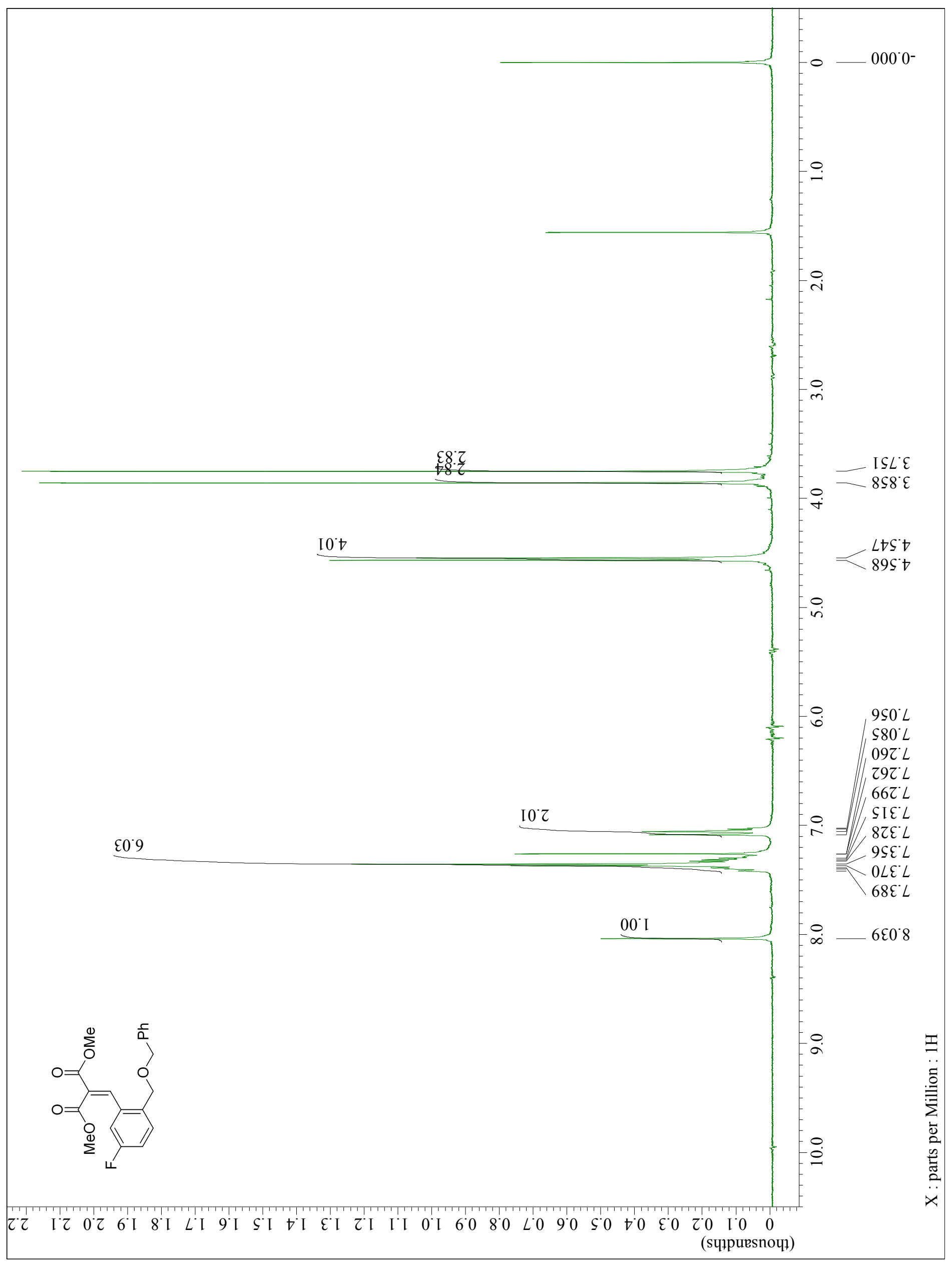


${ }^{13} \mathrm{C}$ NMR spectrum of $\mathbf{3 d}\left(\mathrm{CDCl}_{3}, 75 \mathrm{MHz}\right)$.

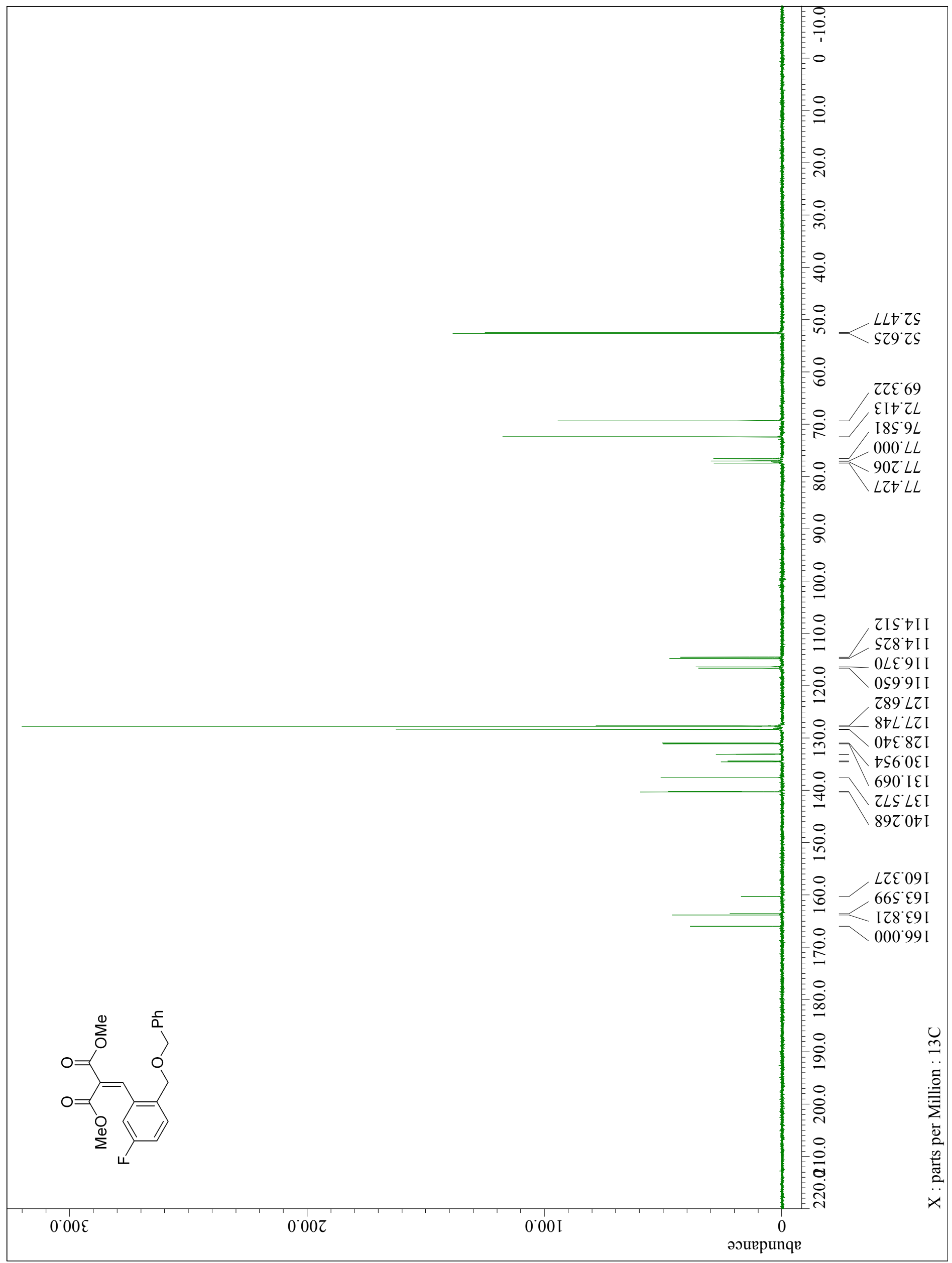


${ }^{19} \mathrm{~F}$ NMR spectrum of $\mathbf{3 d}\left(\mathrm{CDCl}_{3}, 283 \mathrm{MHz}\right)$.

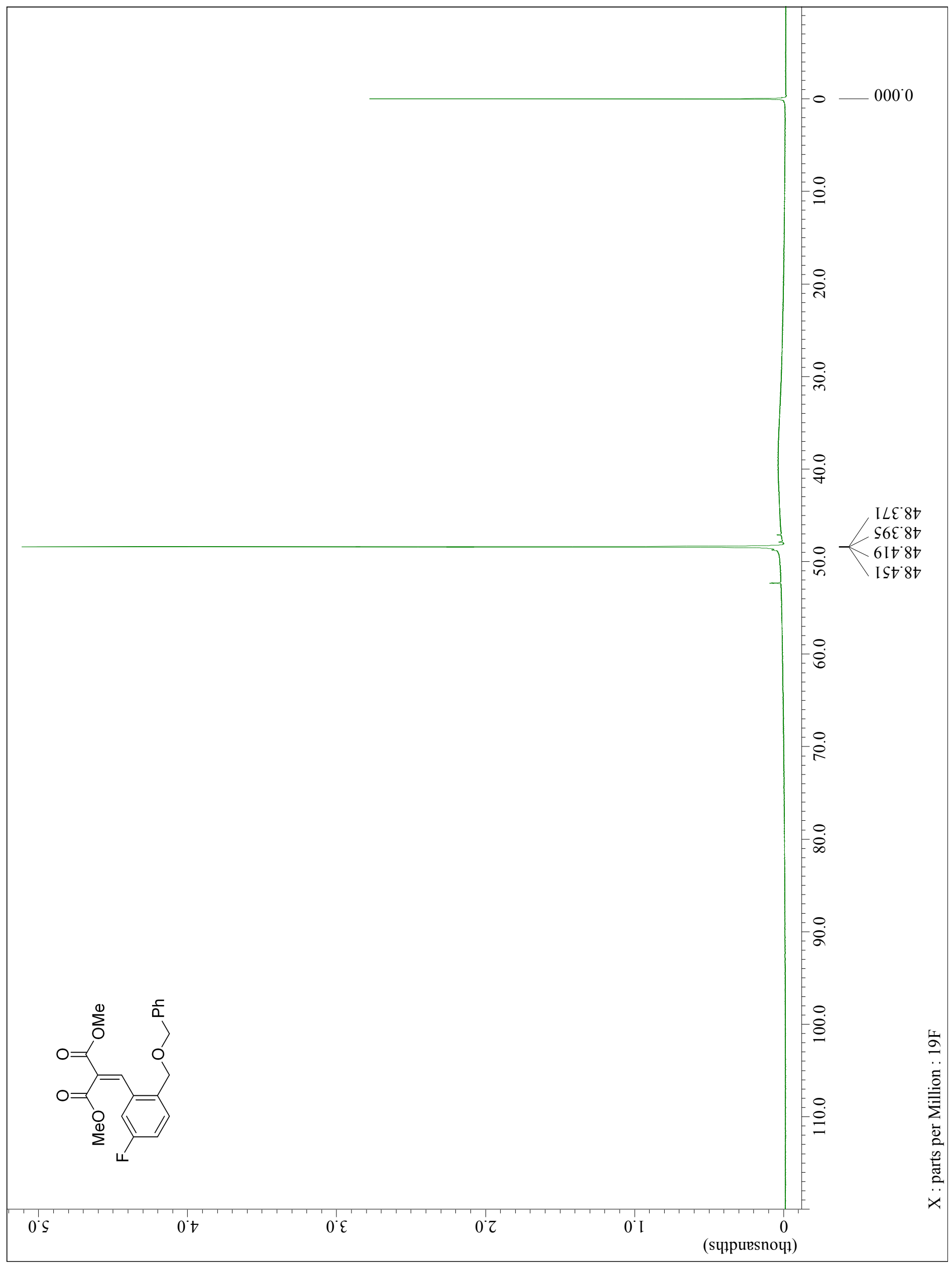


${ }^{1} \mathrm{H}$ NMR spectrum of $\mathbf{s 1 0}\left(\mathrm{CDCl}_{3}, 300 \mathrm{MHz}\right)$.

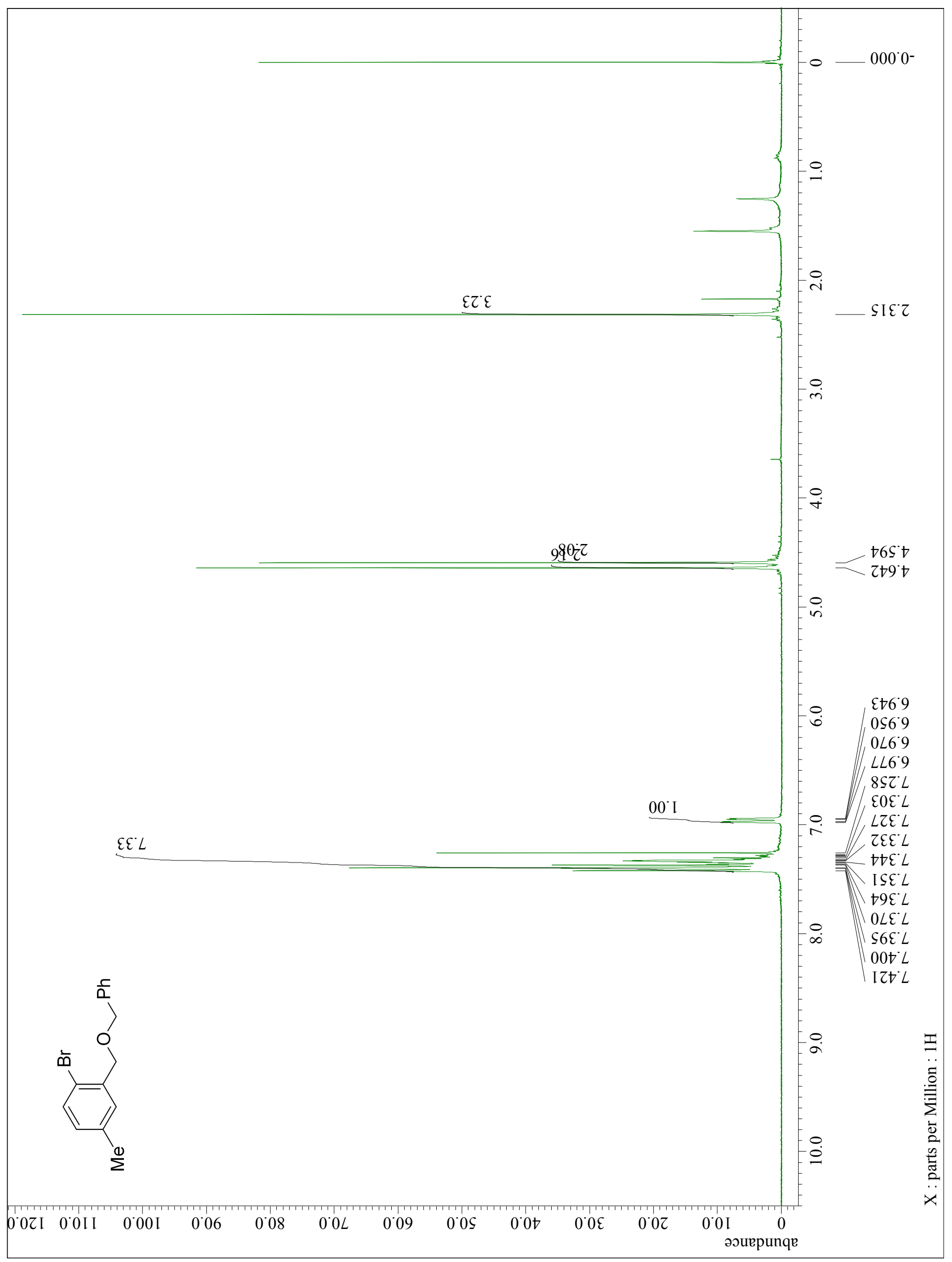




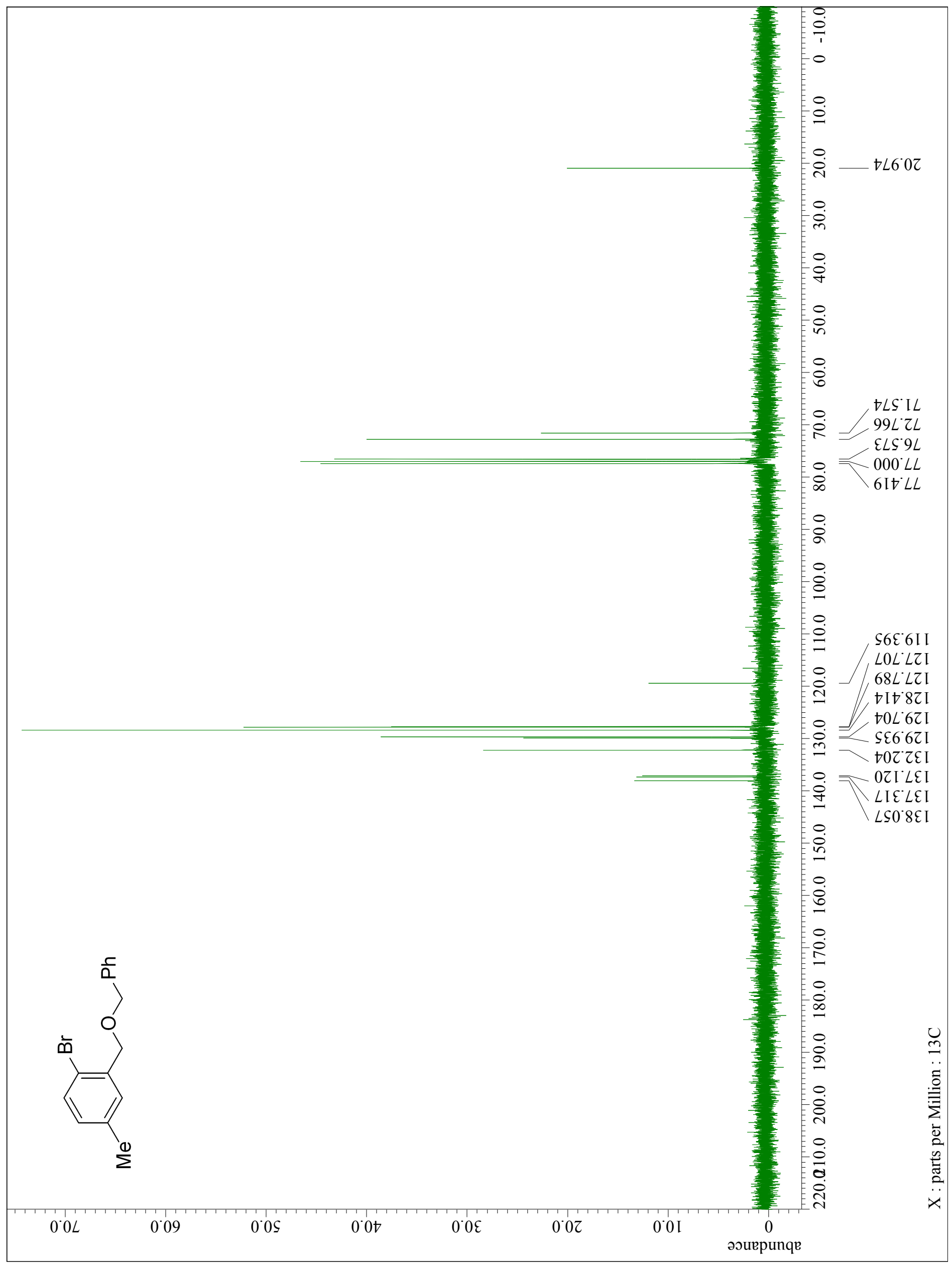


${ }^{1} \mathrm{H}$ NMR spectrum of $\mathbf{s 1 1}\left(\mathrm{CDCl}_{3}, 300 \mathrm{MHz}\right)$.

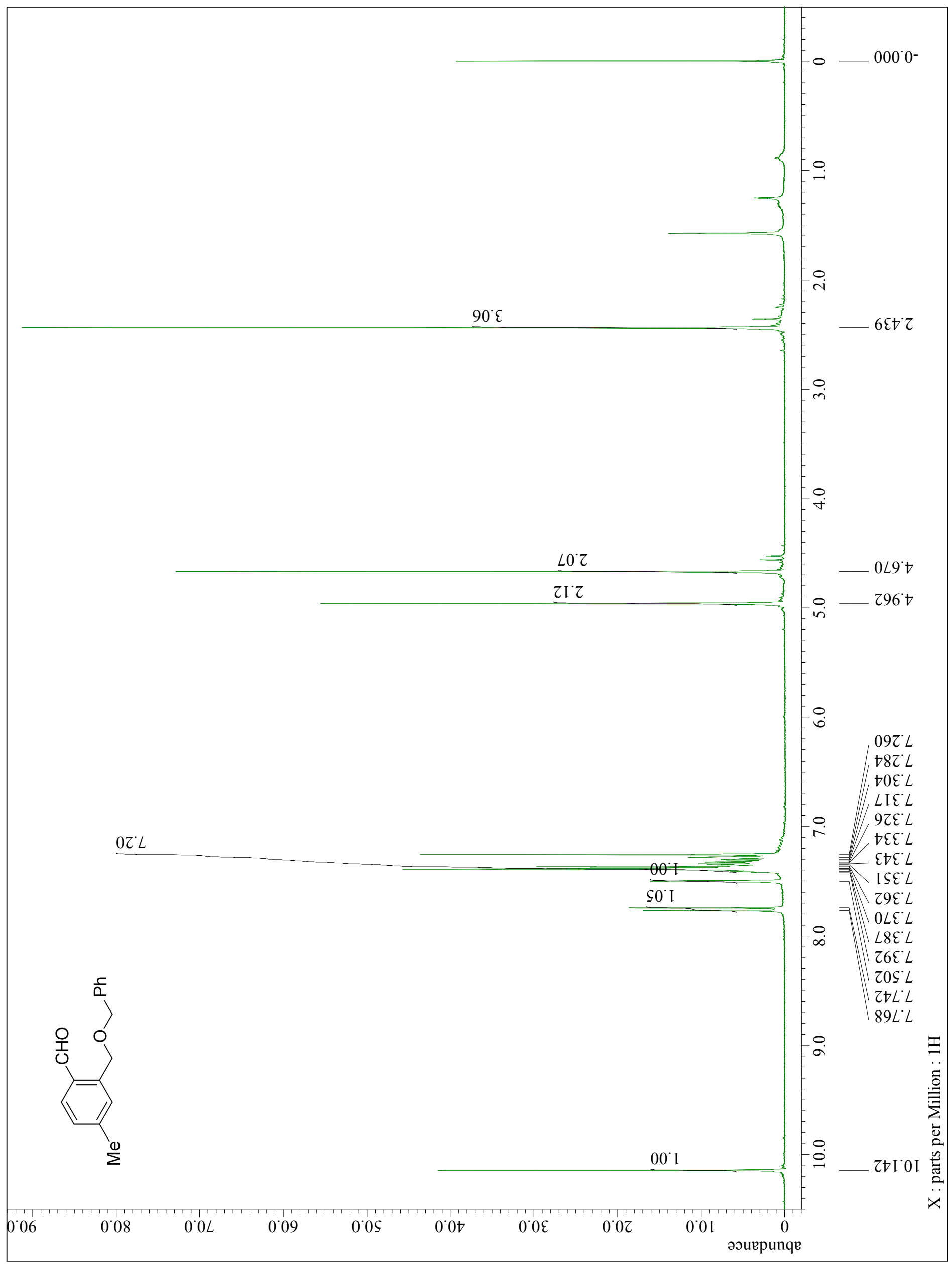


${ }^{13} \mathrm{C} \mathrm{NMR}$ spectrum of $\mathbf{s 1 1}\left(\mathrm{CDCl}_{3}, 75 \mathrm{MHz}\right)$.

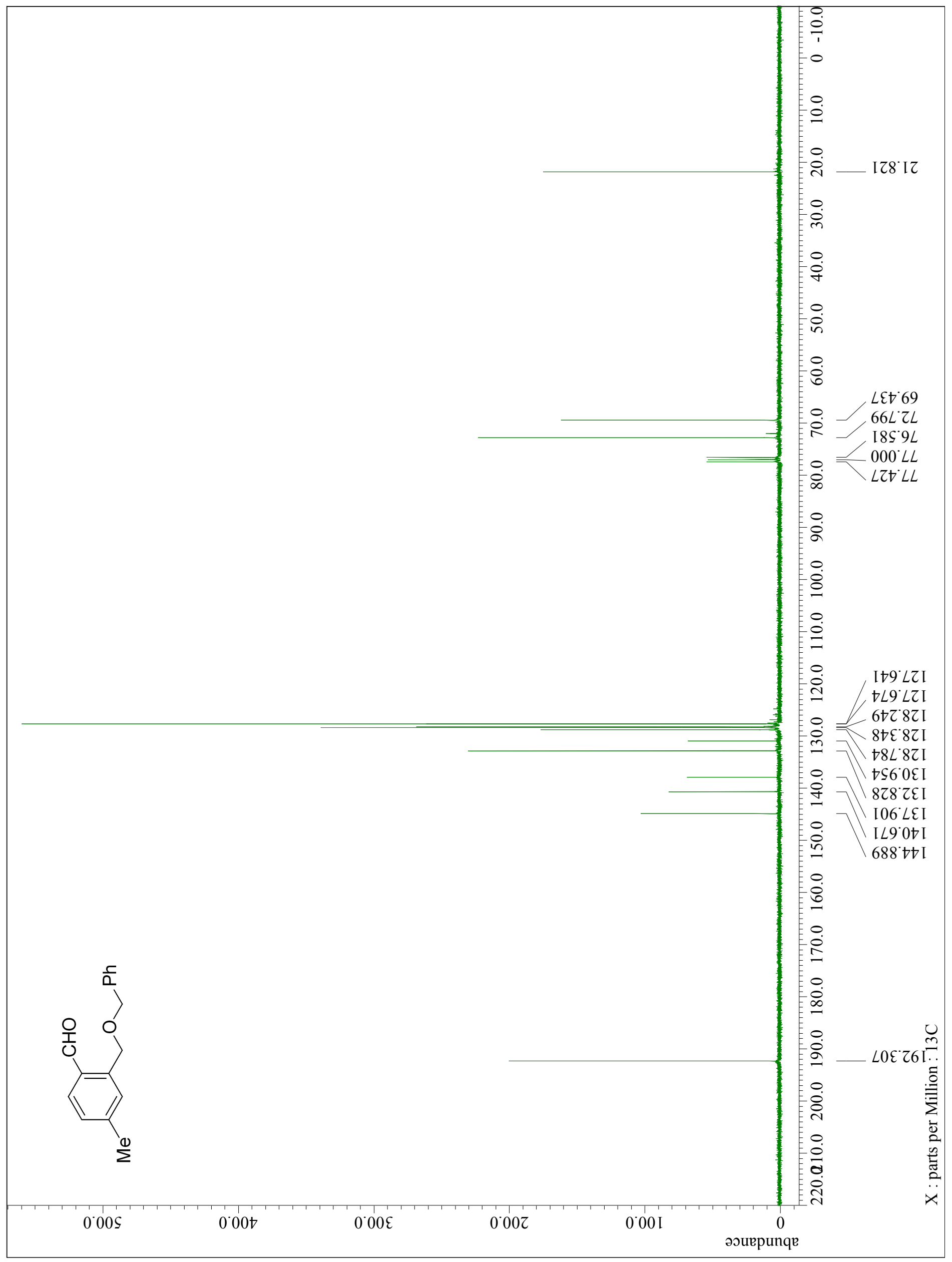


${ }^{1} \mathrm{H}$ NMR spectrum of $\mathbf{3 e}\left(\mathrm{CDCl}_{3}, 300 \mathrm{MHz}\right)$.

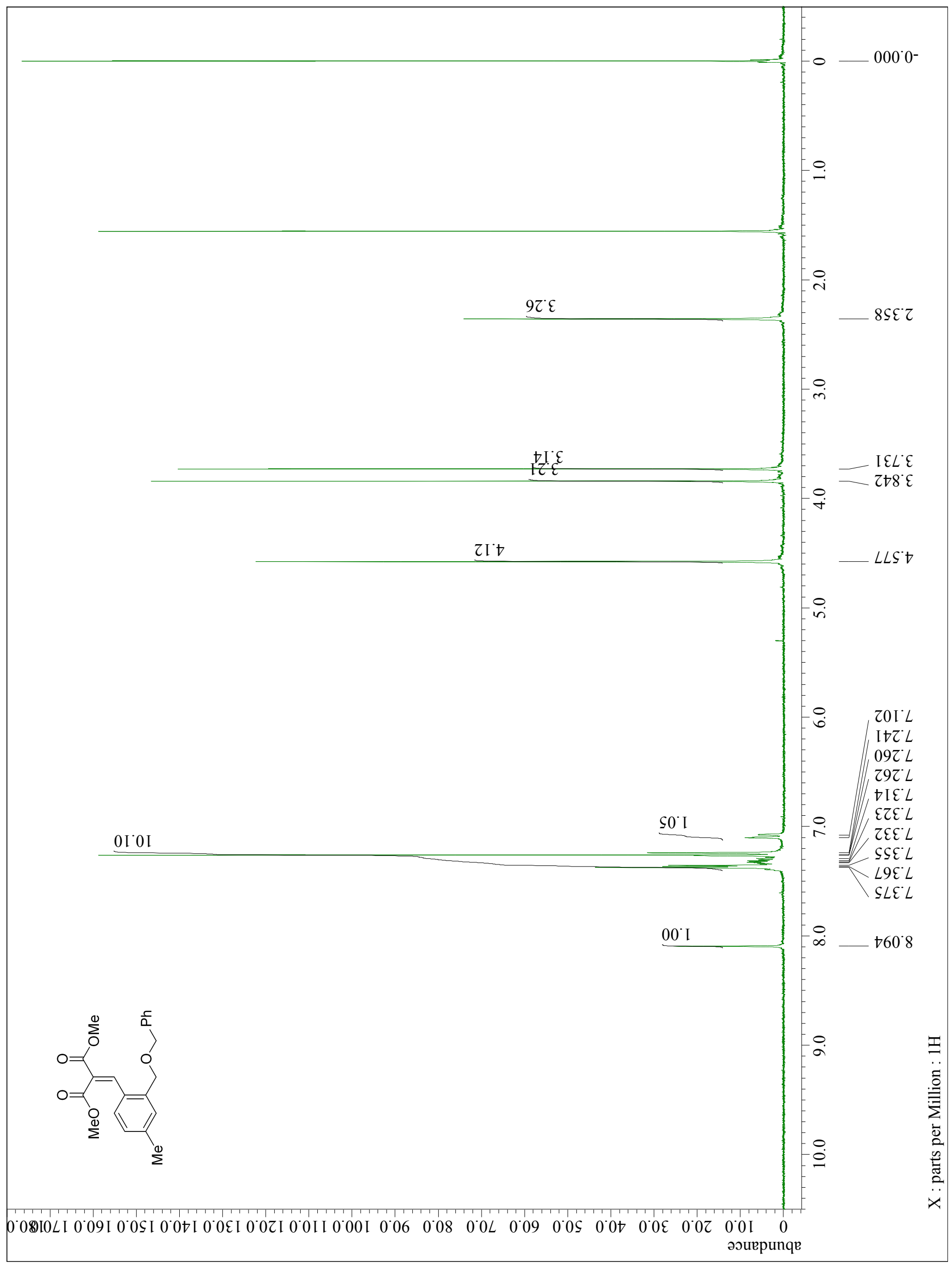


${ }^{13} \mathrm{C} \mathrm{NMR}$ spectrum of $\mathbf{3 e}\left(\mathrm{CDCl}_{3}, 75 \mathrm{MHz}\right)$.

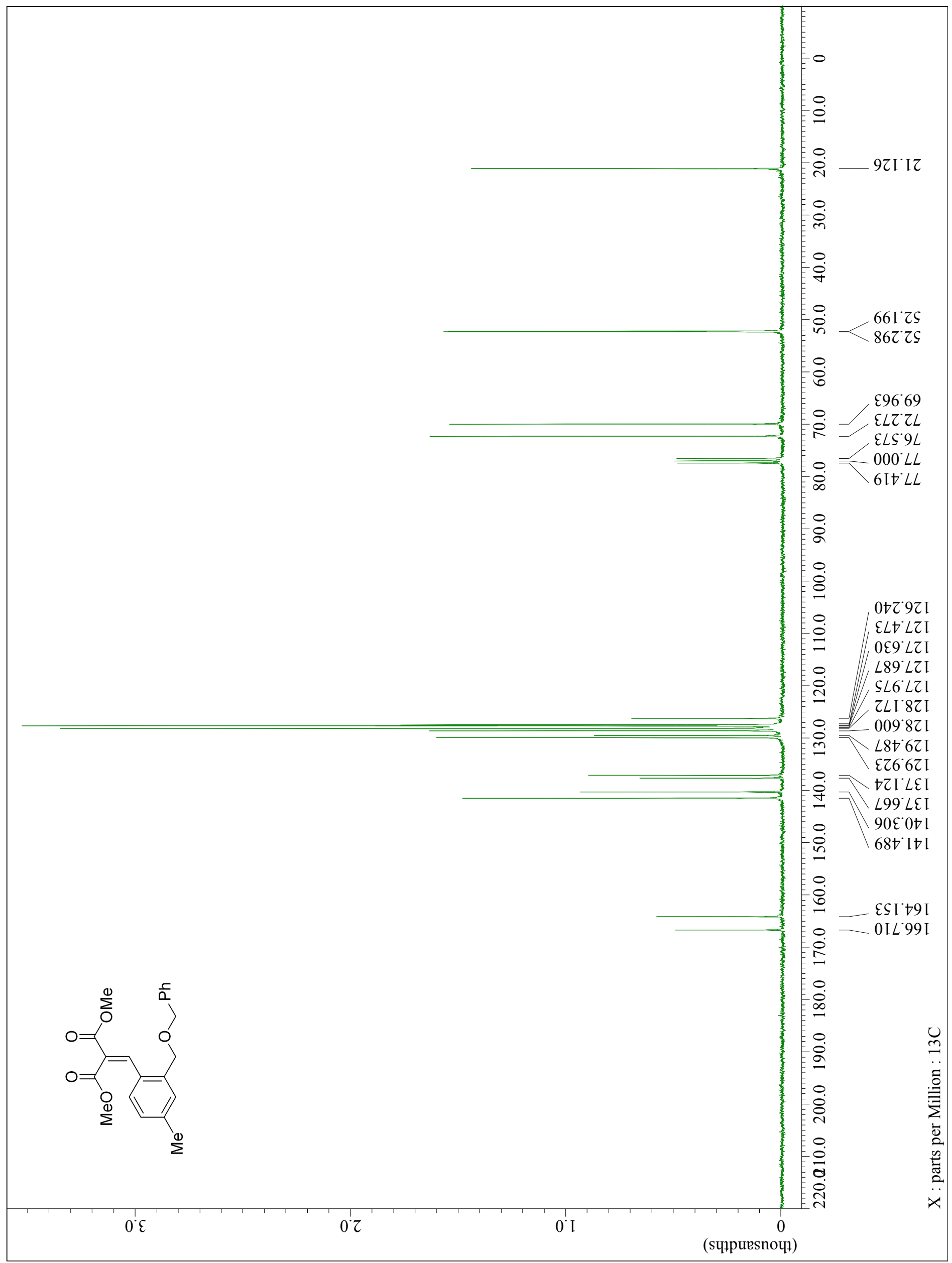


${ }^{1} \mathrm{H}$ NMR spectrum of $\mathbf{s 1 2}\left(\mathrm{CDCl}_{3}, 300 \mathrm{MHz}\right)$.

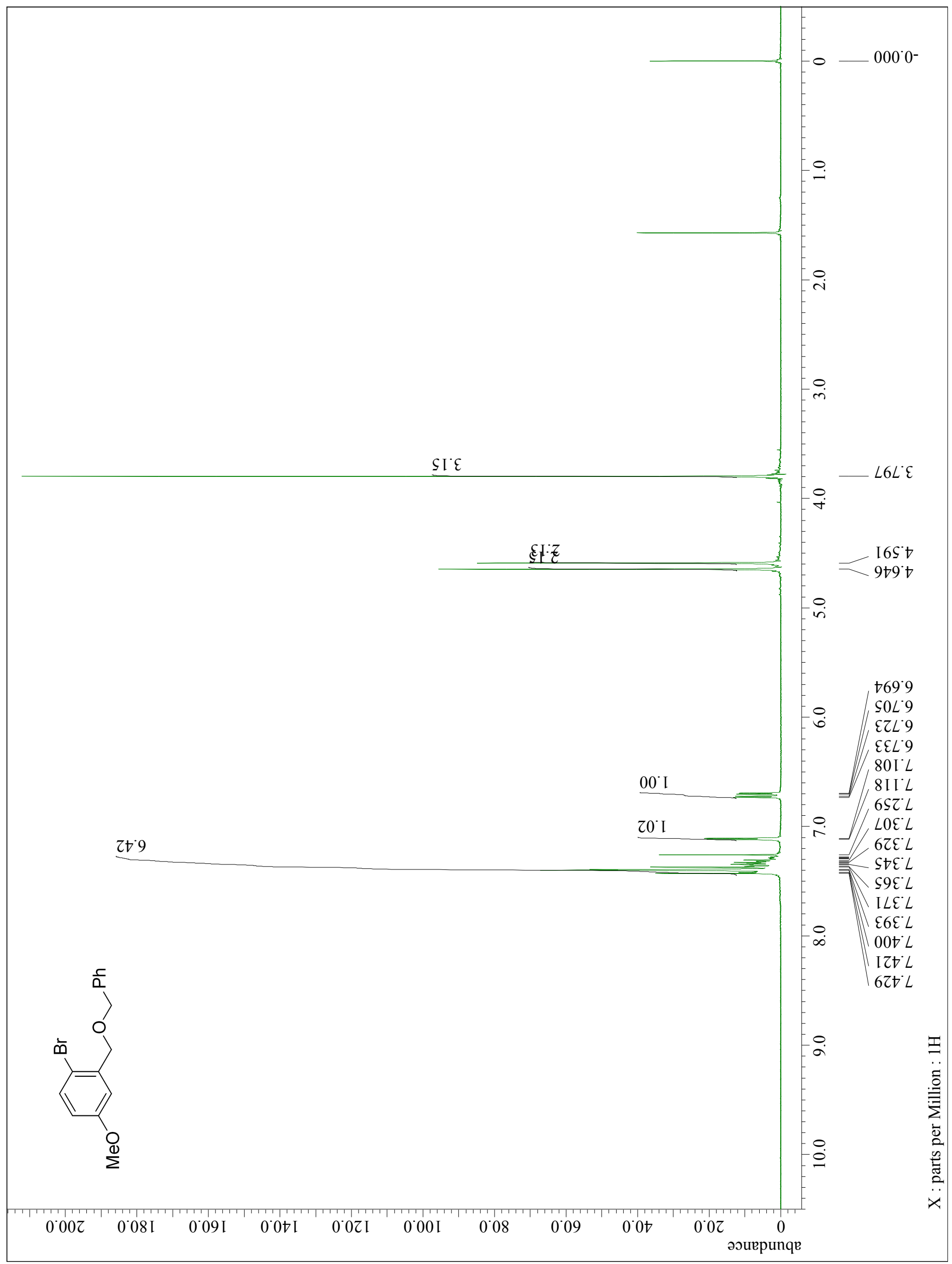


${ }^{13} \mathrm{C}$ NMR spectrum of $\mathbf{s 1 2}\left(\mathrm{CDCl}_{3}, 75 \mathrm{MHz}\right)$.

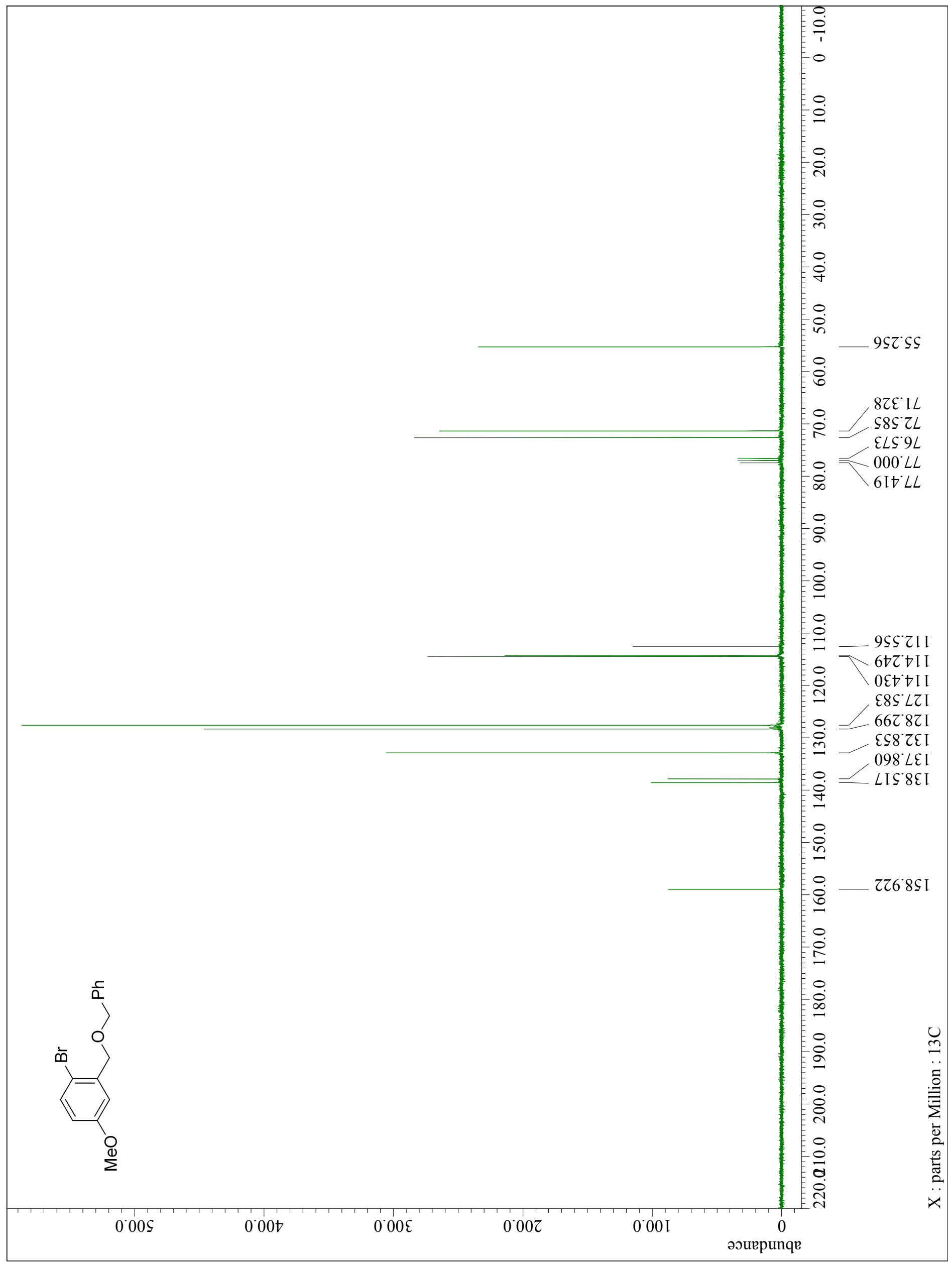


${ }^{1} \mathrm{H}$ NMR spectrum of $\mathbf{s 1 3}\left(\mathrm{CDCl}_{3}, 300 \mathrm{MHz}\right)$.

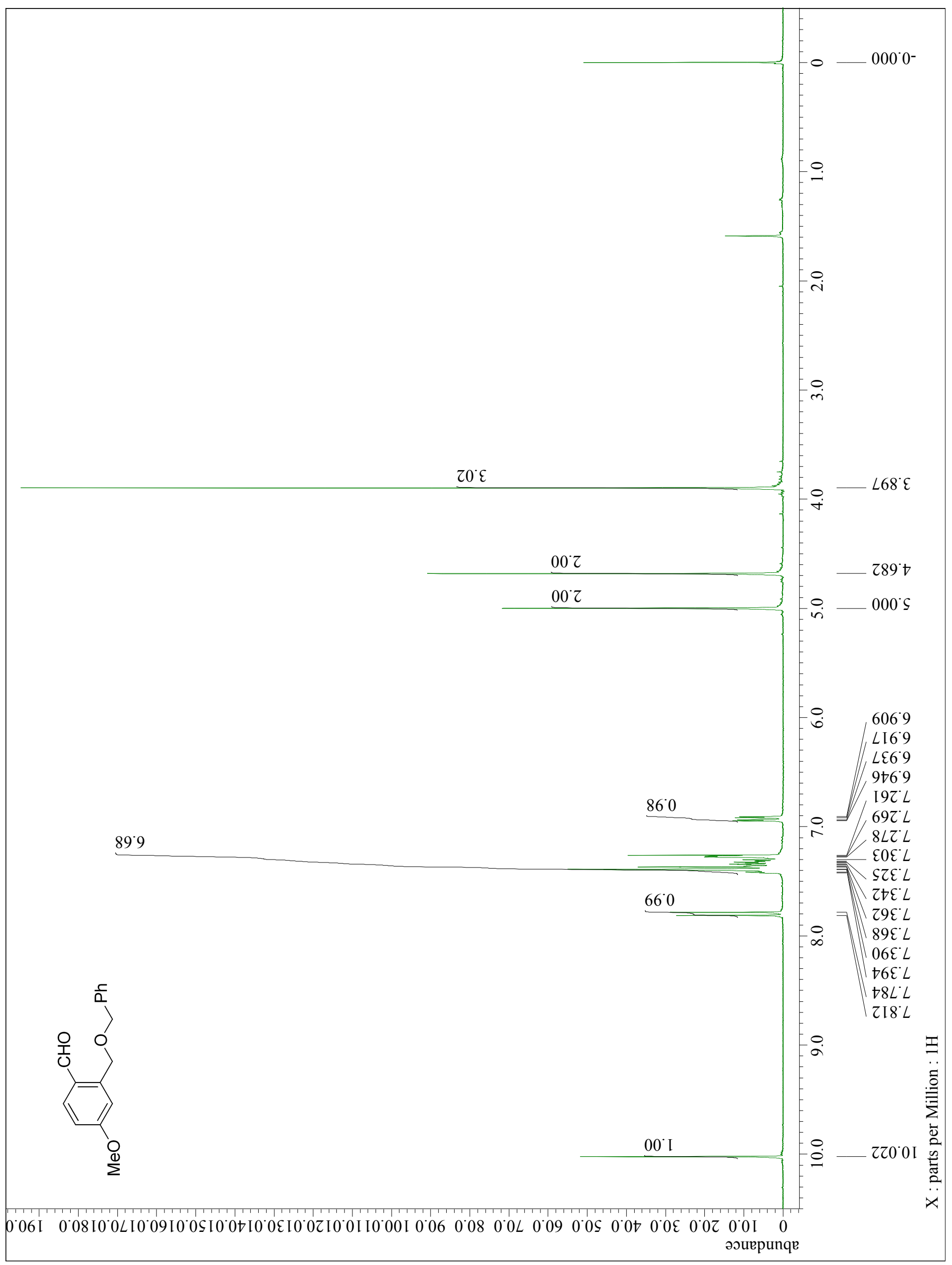




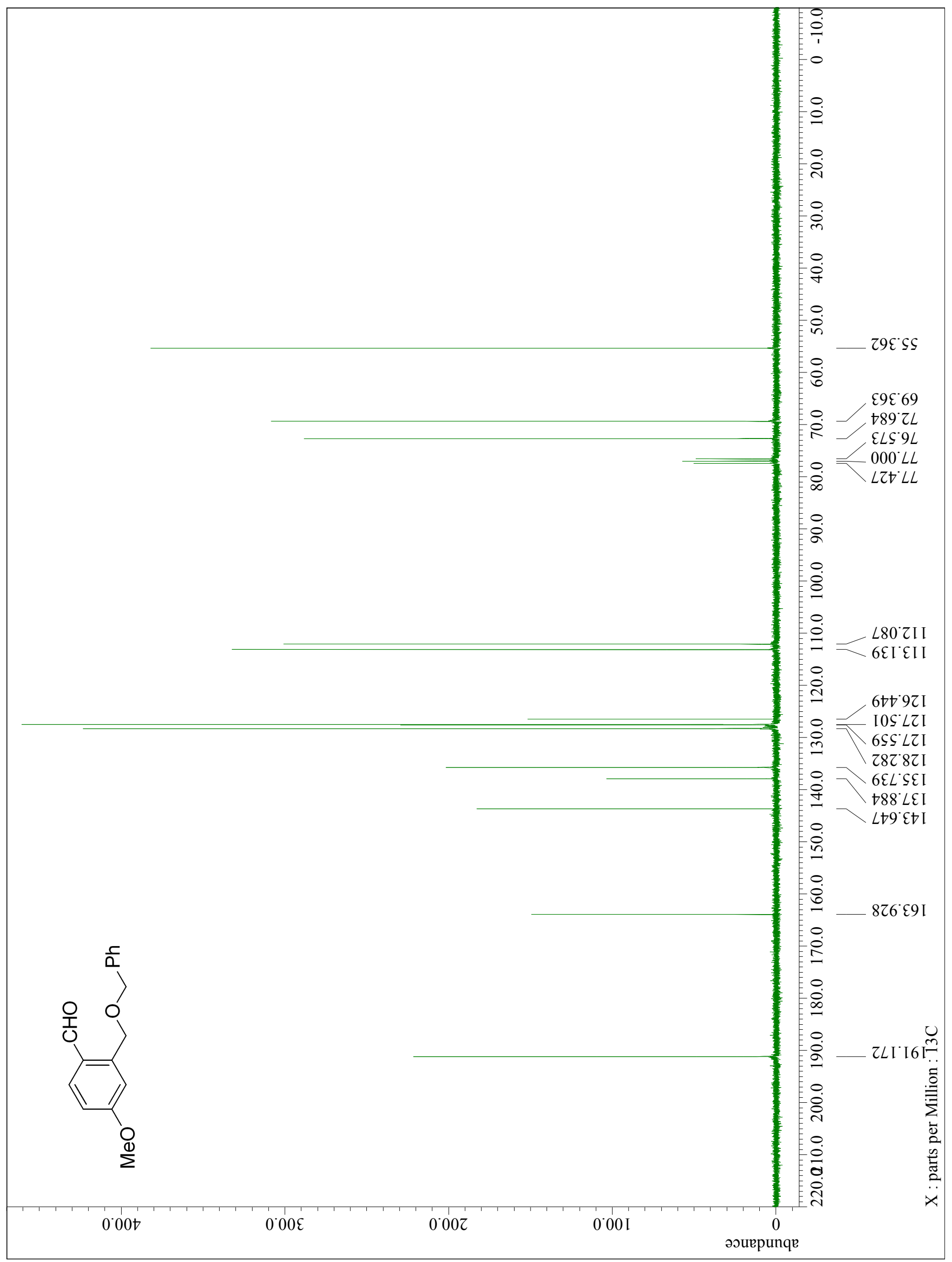


${ }^{1} \mathrm{H}$ NMR spectrum of $\mathbf{3 f}\left(\mathrm{CDCl}_{3}, 300 \mathrm{MHz}\right)$.

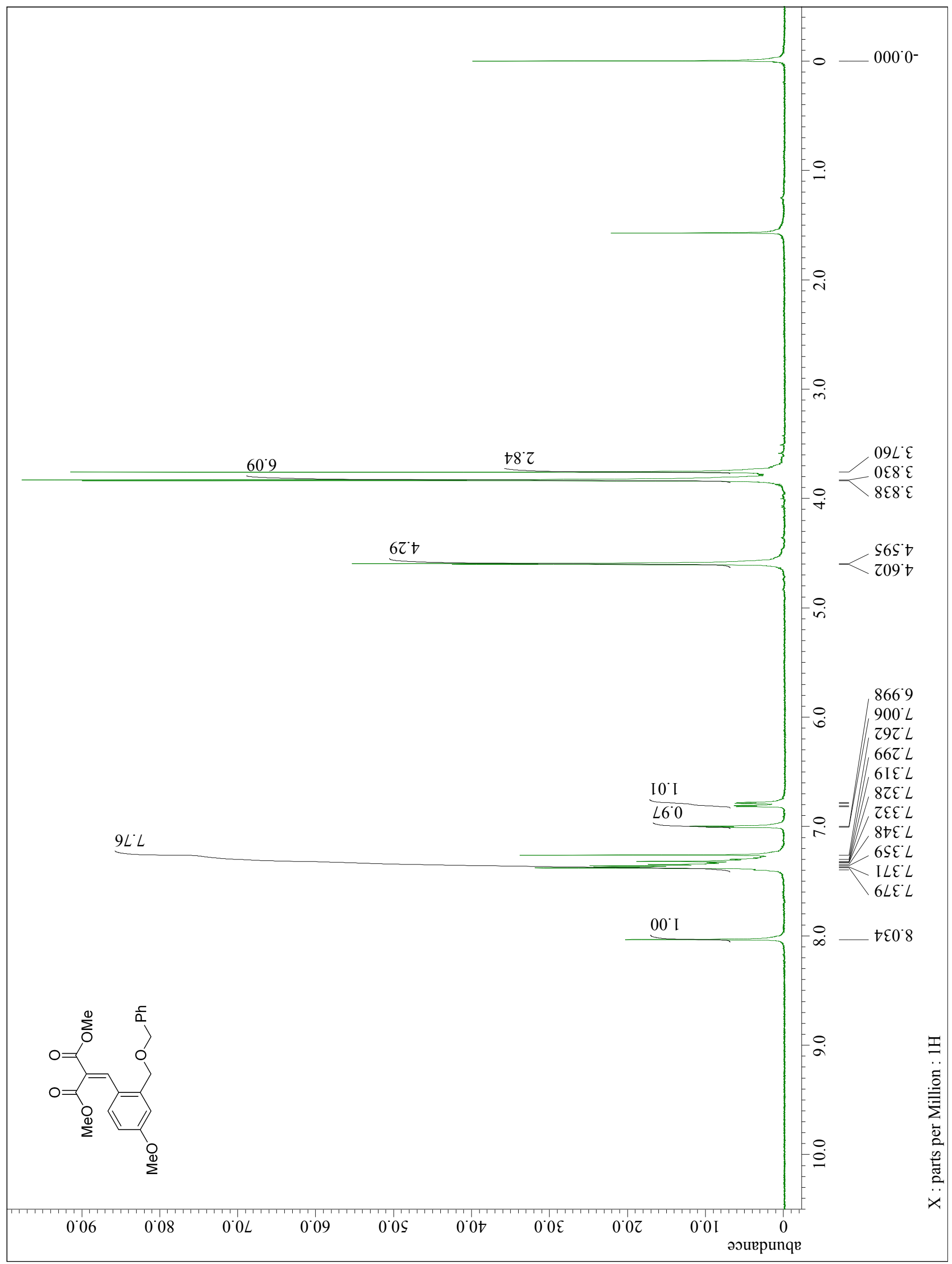


${ }^{13} \mathrm{C}$ NMR spectrum of $\mathbf{3 f}\left(\mathrm{CDCl}_{3}, 75 \mathrm{MHz}\right)$.

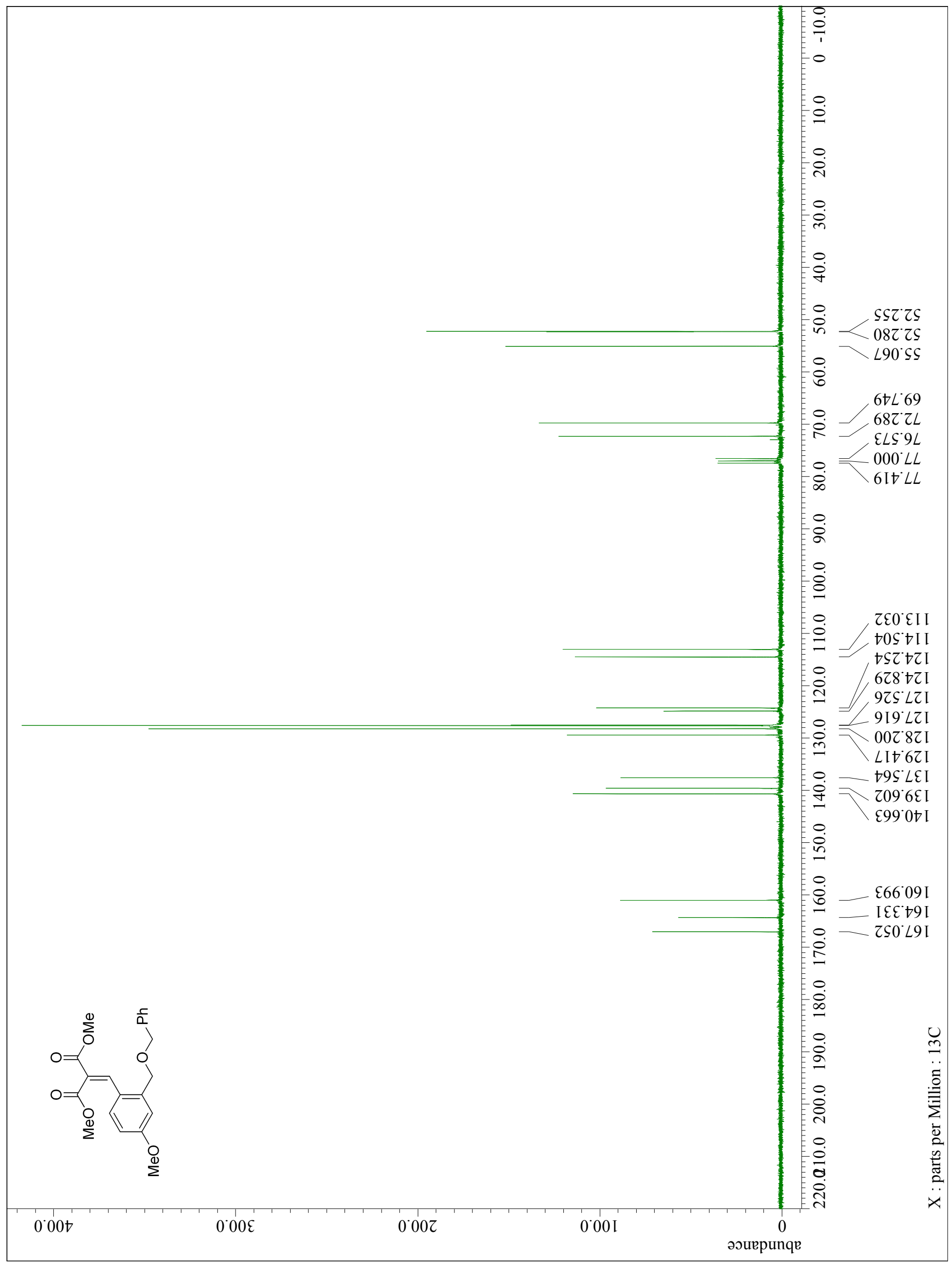


${ }^{1} \mathrm{H}$ NMR spectrum of $\mathbf{s 1 4}\left(\mathrm{CDCl}_{3}, 300 \mathrm{MHz}\right)$.

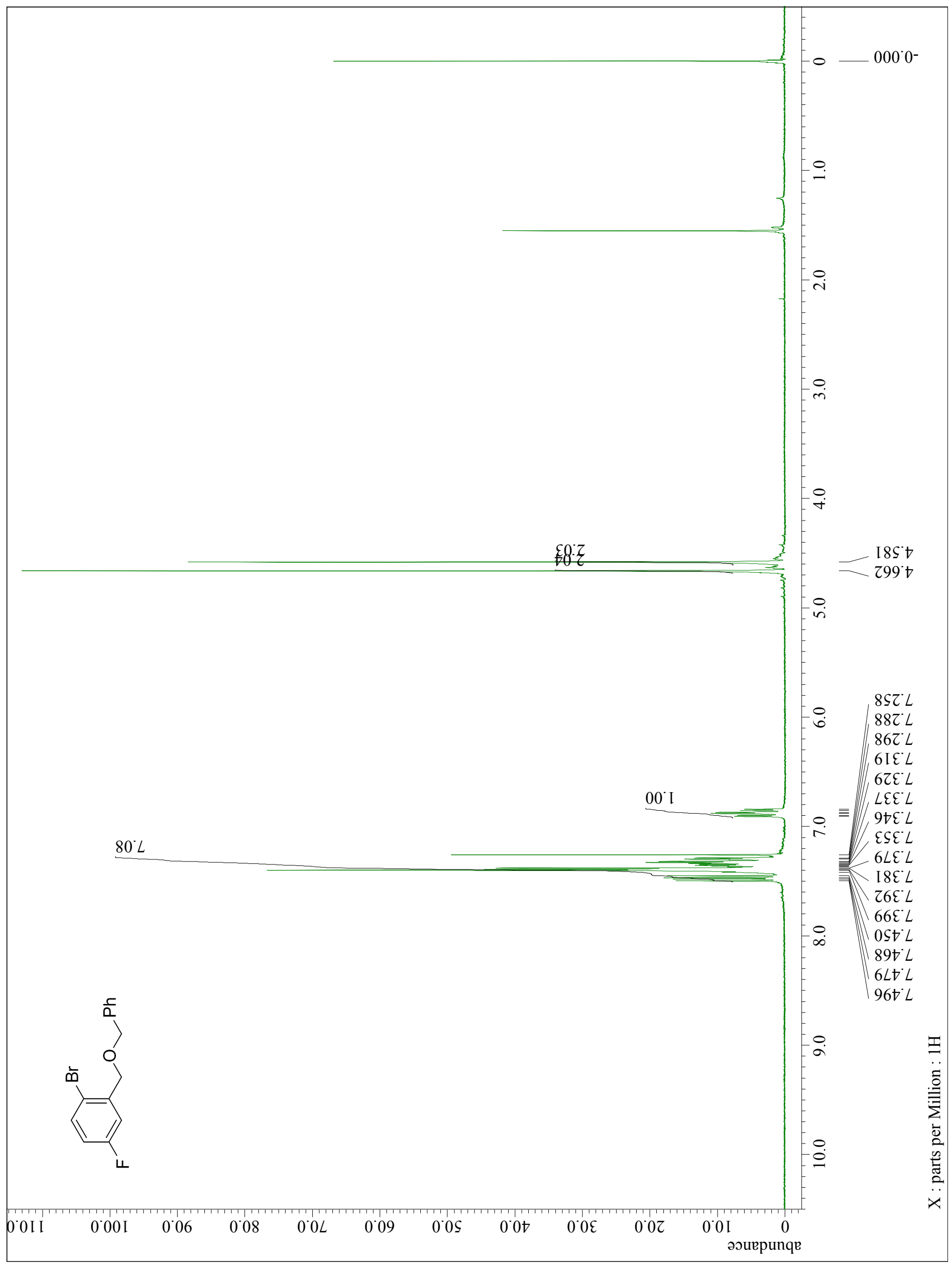


${ }^{13} \mathrm{C}$ NMR spectrum of $\mathbf{s} 14\left(\mathrm{CDCl}_{3}, 75 \mathrm{MHz}\right)$.

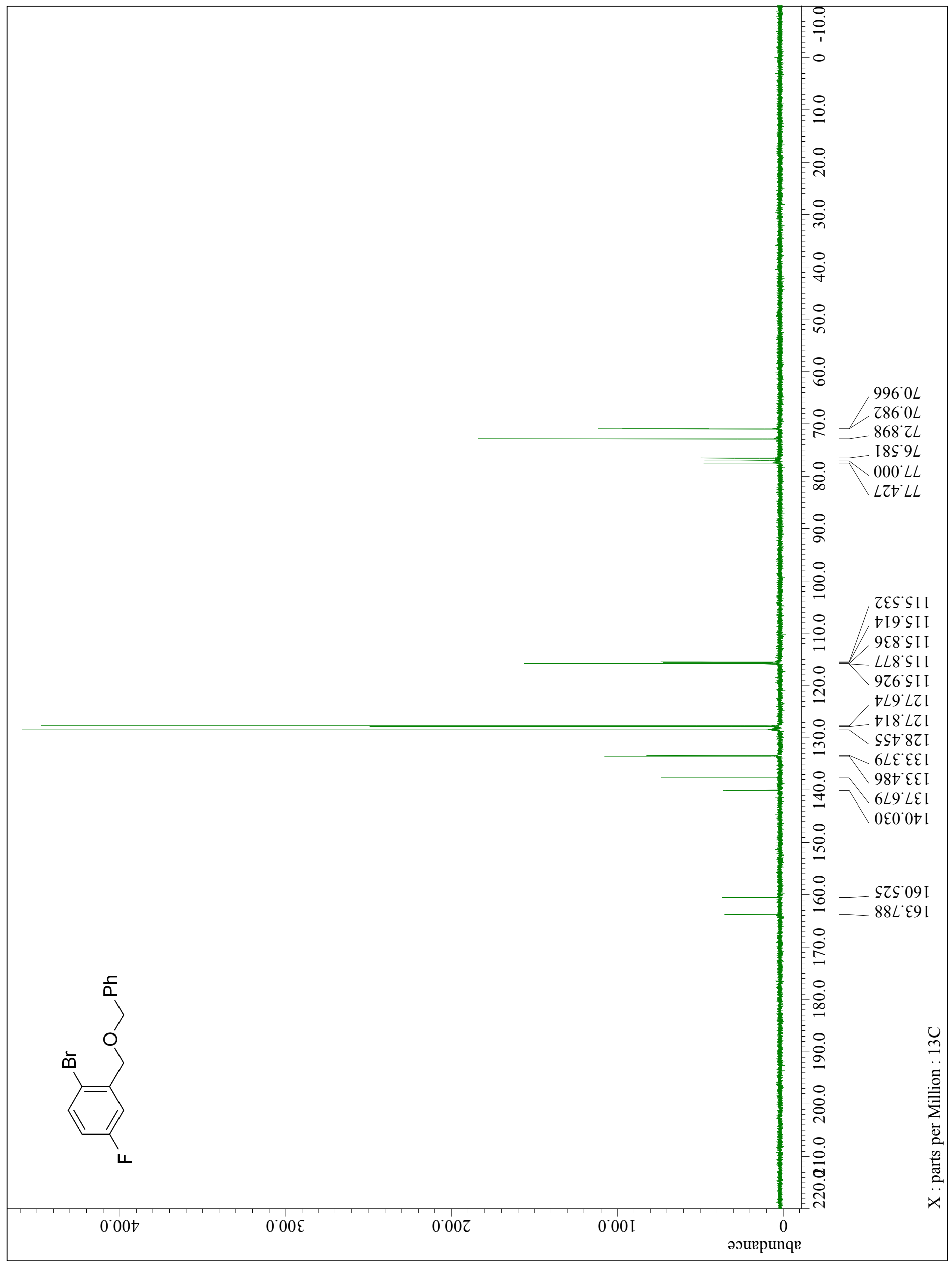


${ }^{19} \mathrm{~F}$ NMR spectrum of $\mathbf{s 1 4}\left(\mathrm{CDCl}_{3}, 283 \mathrm{MHz}\right)$.

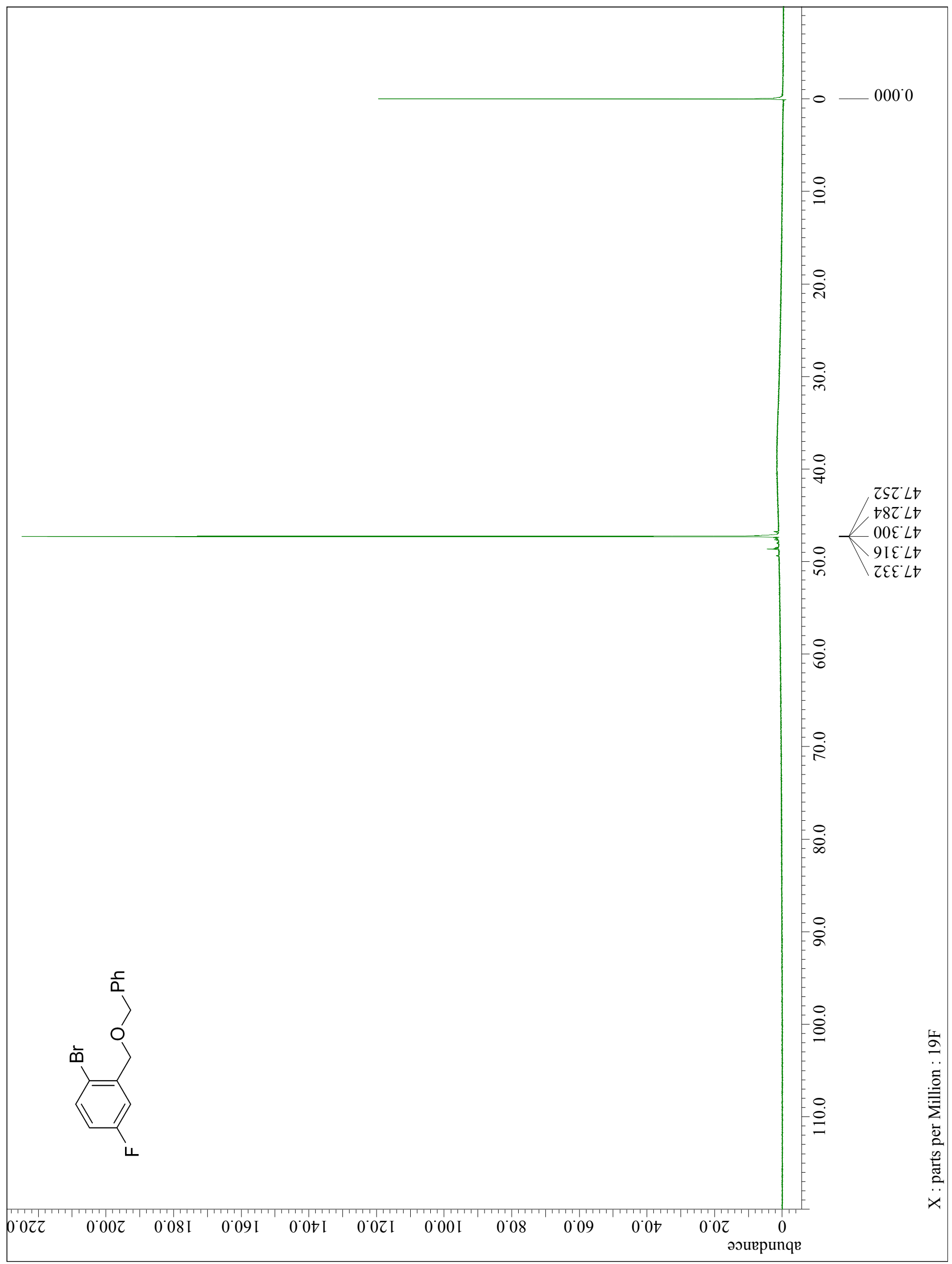


${ }^{1} \mathrm{H}$ NMR spectrum of $\mathbf{s 1 5}\left(\mathrm{CDCl}_{3}, 300 \mathrm{MHz}\right)$.

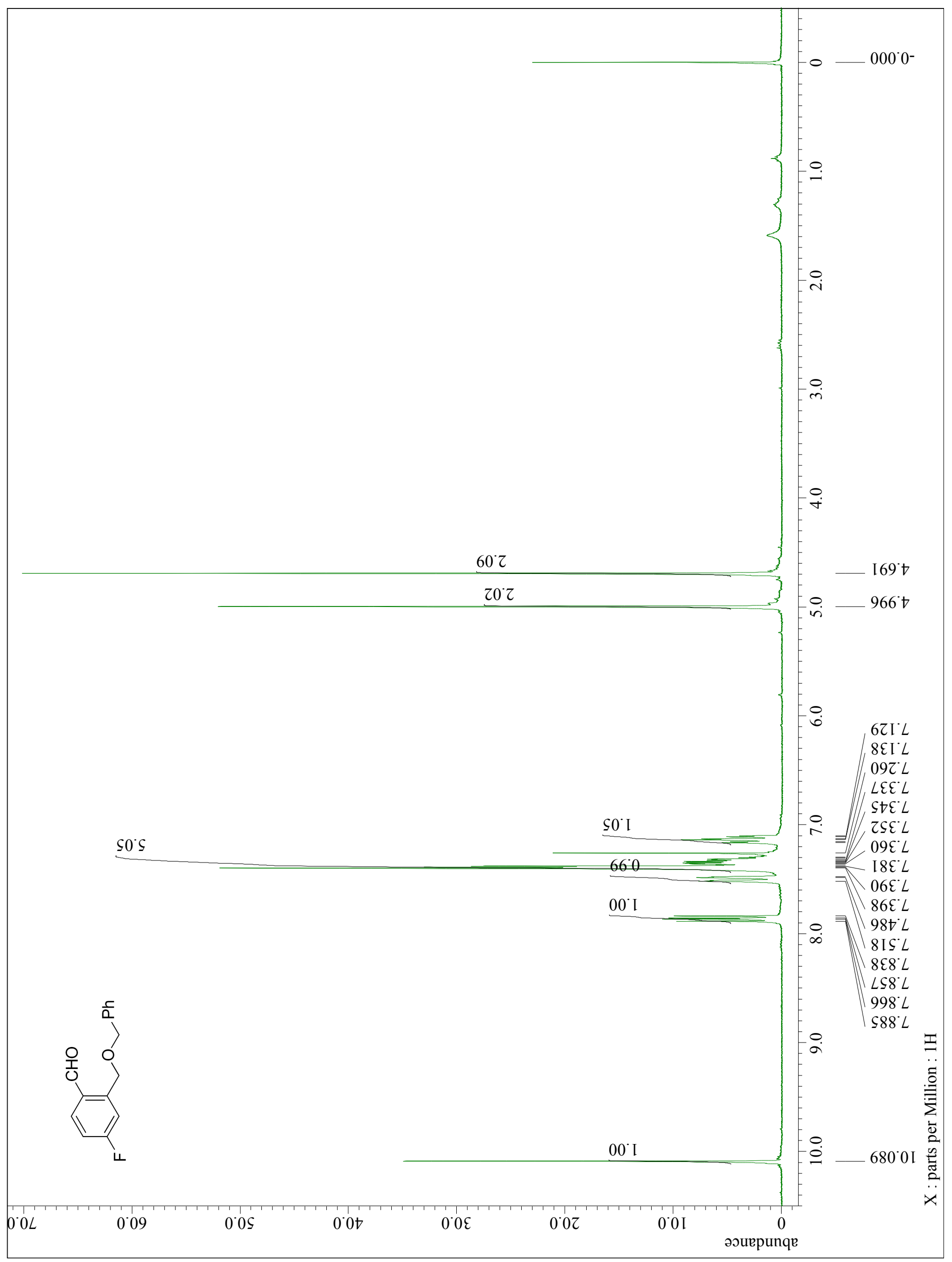




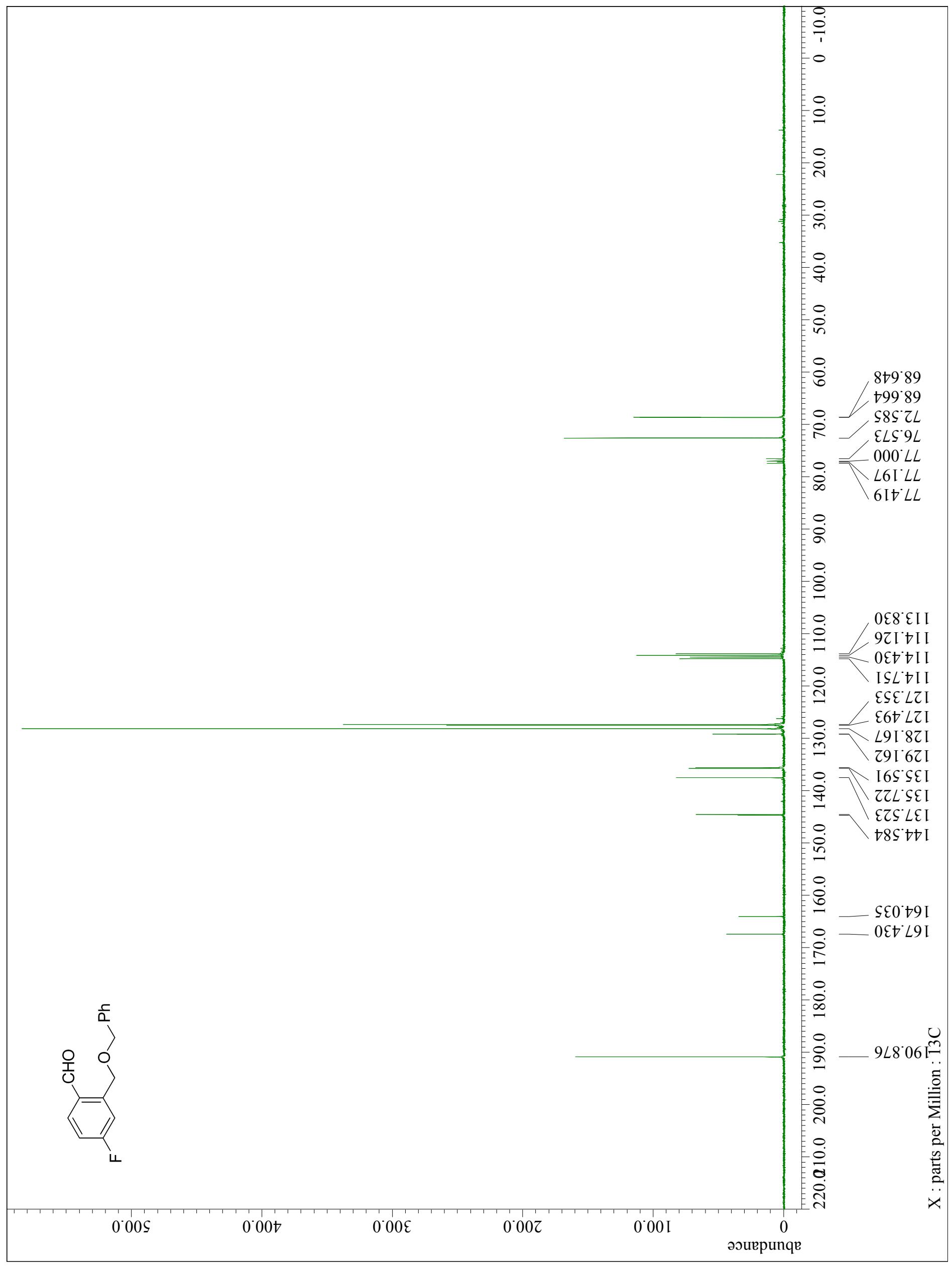


${ }^{19} \mathrm{~F}$ NMR spectrum of $\mathbf{s 1 5}\left(\mathrm{CDCl}_{3}, 283 \mathrm{MHz}\right)$.

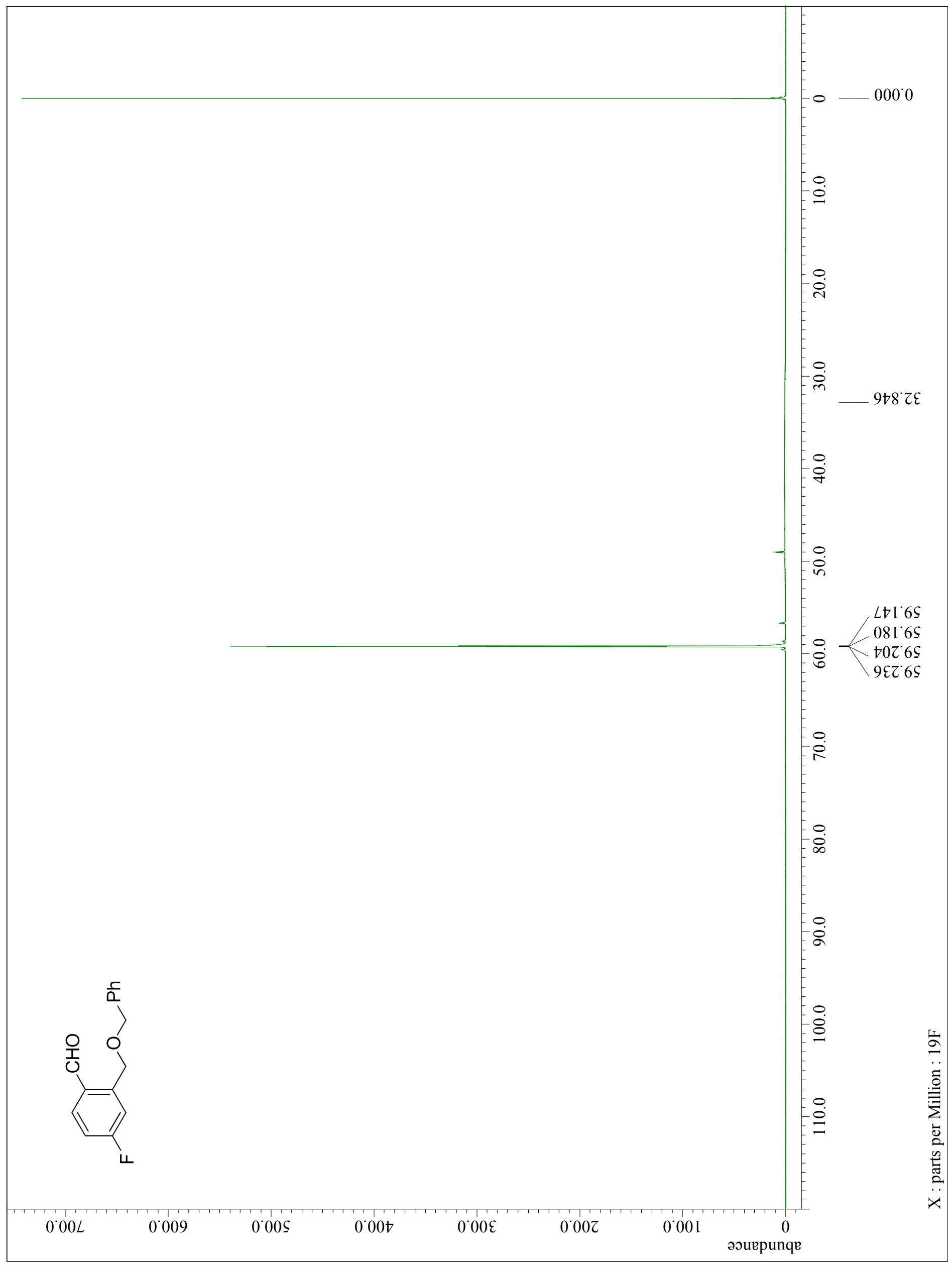


${ }^{1} \mathrm{H}$ NMR spectrum of $\mathbf{3 g}\left(\mathrm{CDCl}_{3}, 300 \mathrm{MHz}\right)$.

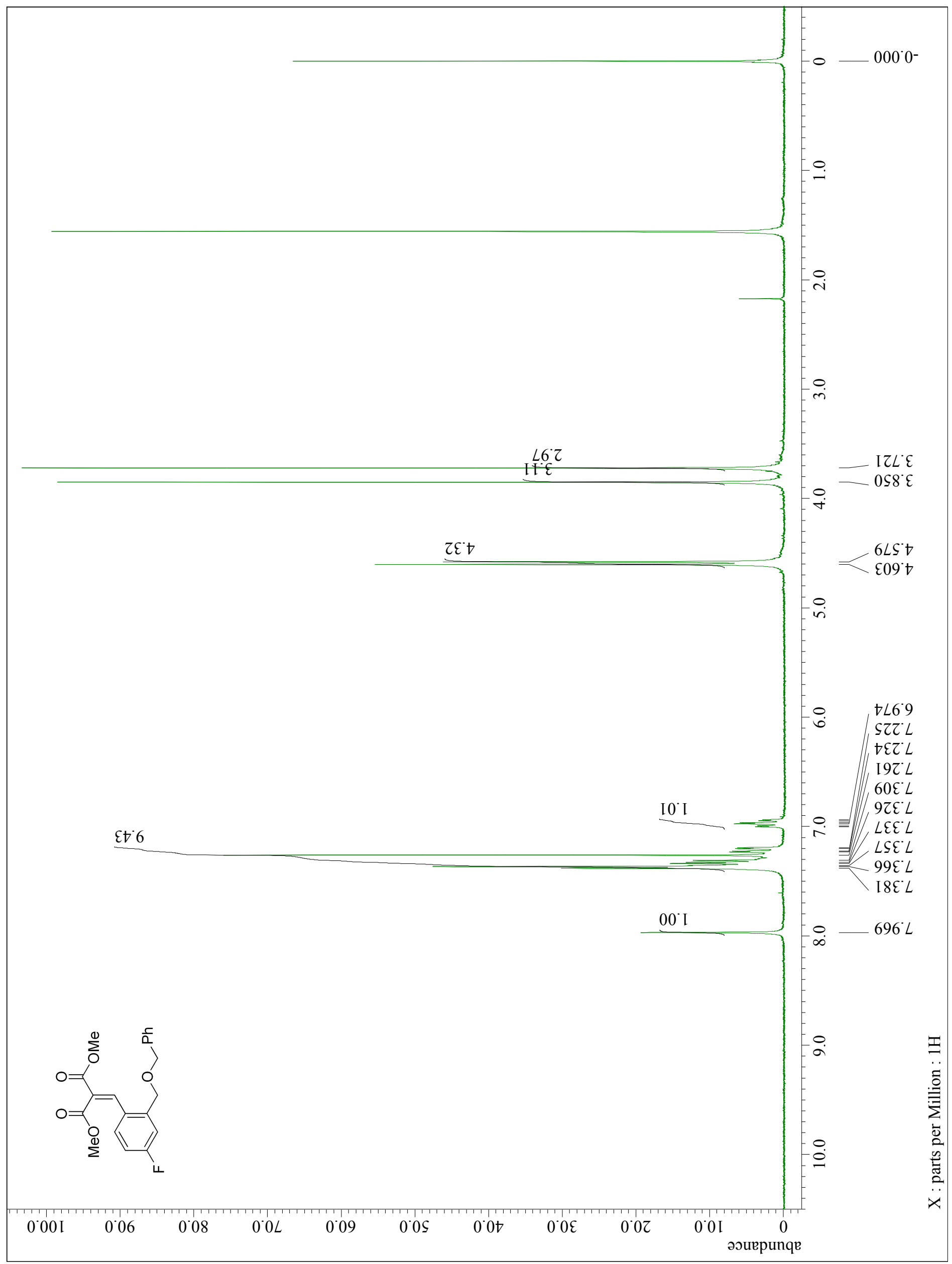


${ }^{13} \mathrm{C} \mathrm{NMR}$ spectrum of $\mathbf{3 g}\left(\mathrm{CDCl}_{3}, 75 \mathrm{MHz}\right)$.

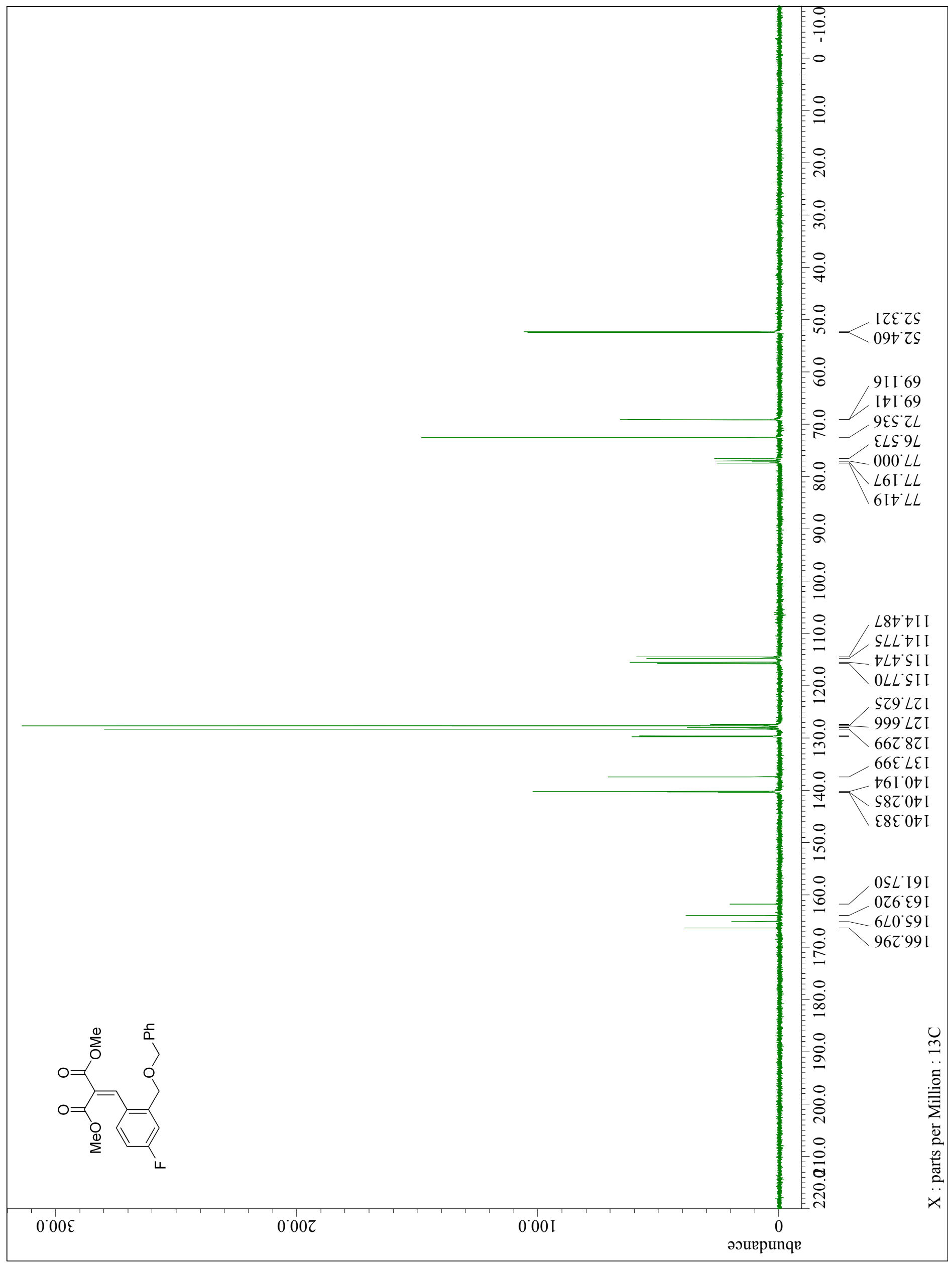


${ }^{19} \mathrm{~F}$ NMR spectrum of $\mathbf{3 g}\left(\mathrm{CDCl}_{3}, 283 \mathrm{MHz}\right)$.

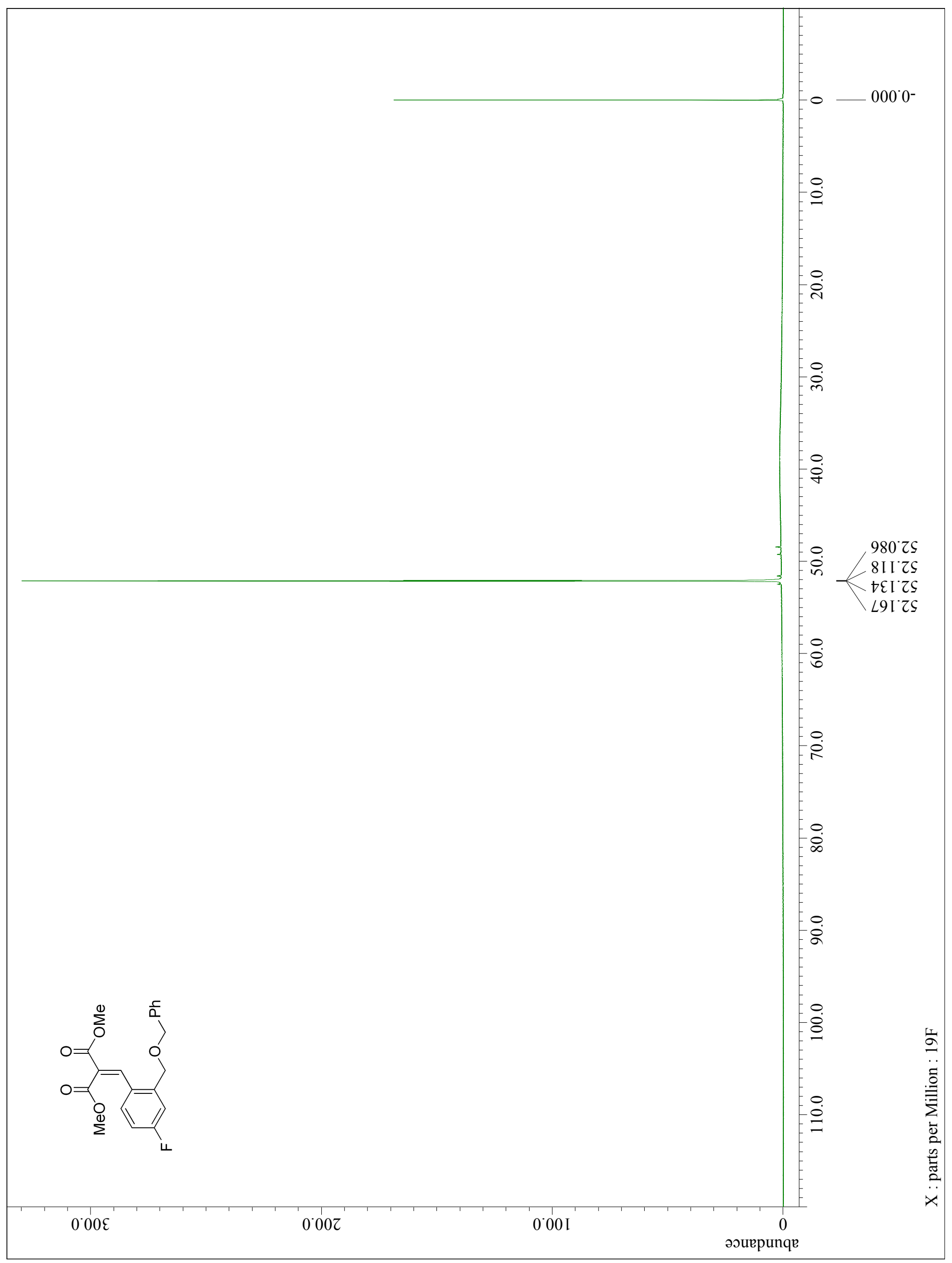


${ }^{1} \mathrm{H}$ NMR spectrum of $\mathbf{s 1 6}\left(\mathrm{CDCl}_{3}, 300 \mathrm{MHz}\right)$.

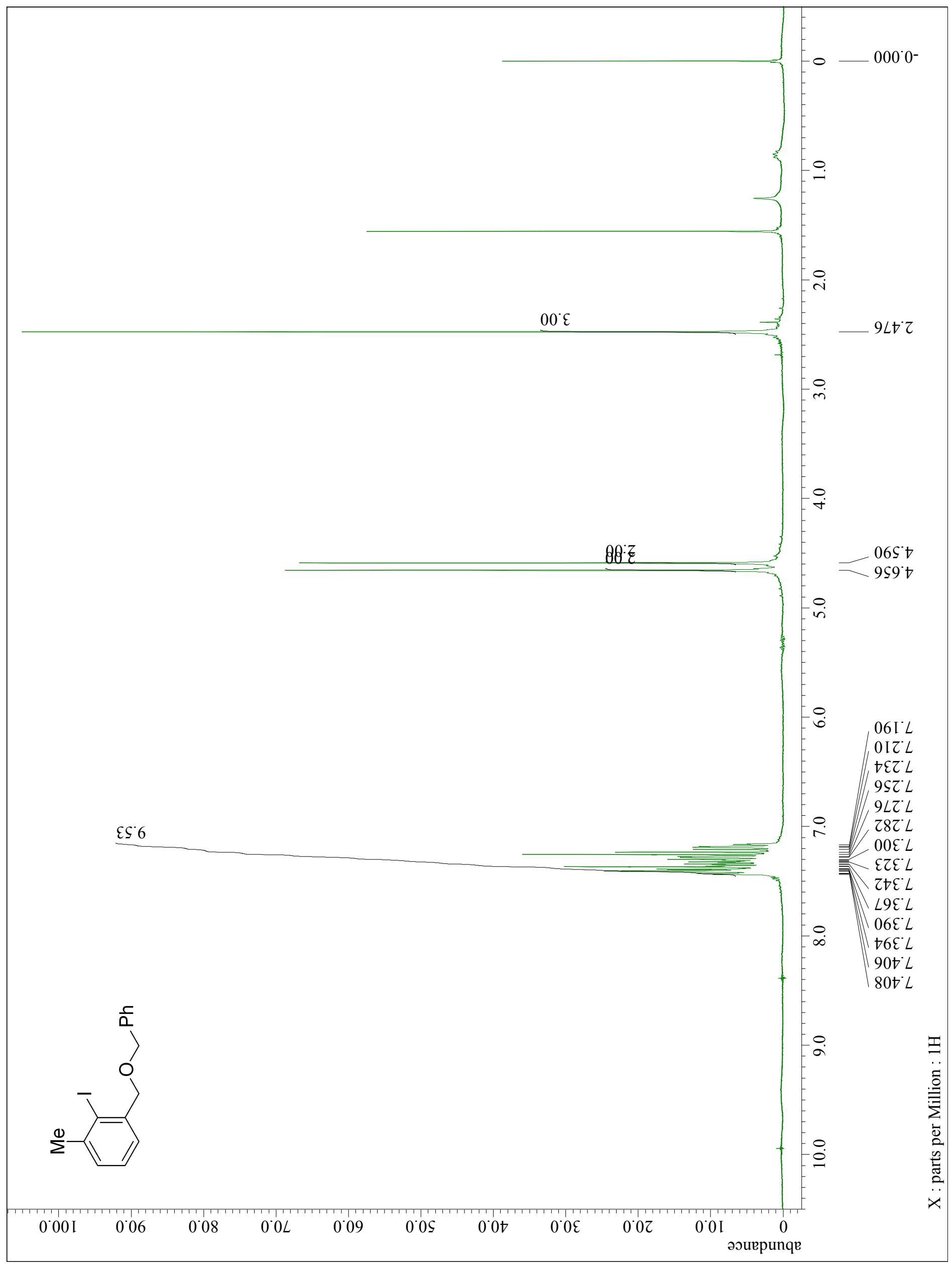


${ }^{13} \mathrm{C}$ NMR spectrum of $\mathbf{s 1 6}\left(\mathrm{CDCl}_{3}, 75 \mathrm{MHz}\right)$.

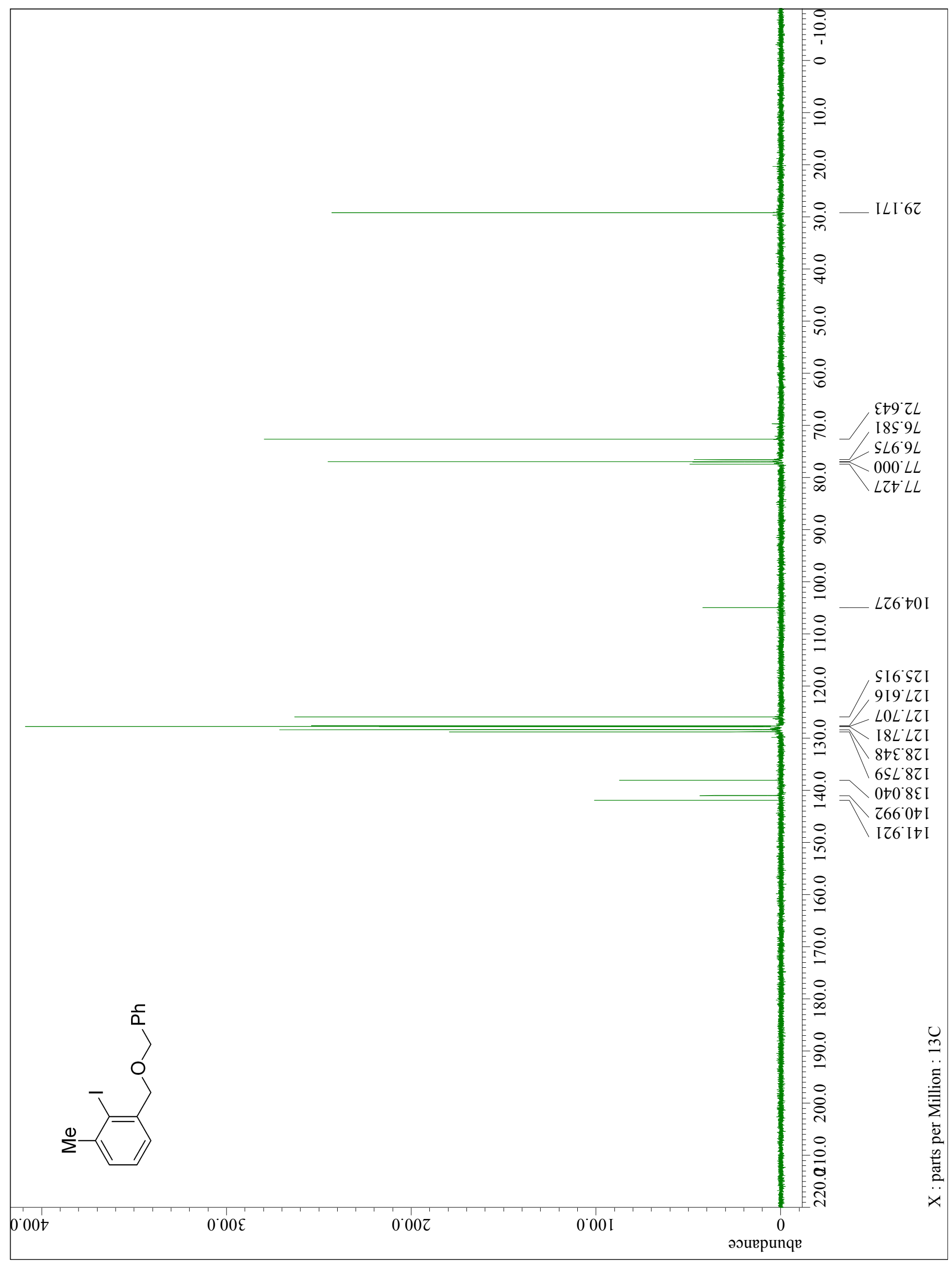


${ }^{1} \mathrm{H}$ NMR spectrum of $\mathbf{s 1 7}\left(\mathrm{CDCl}_{3}, 300 \mathrm{MHz}\right)$.

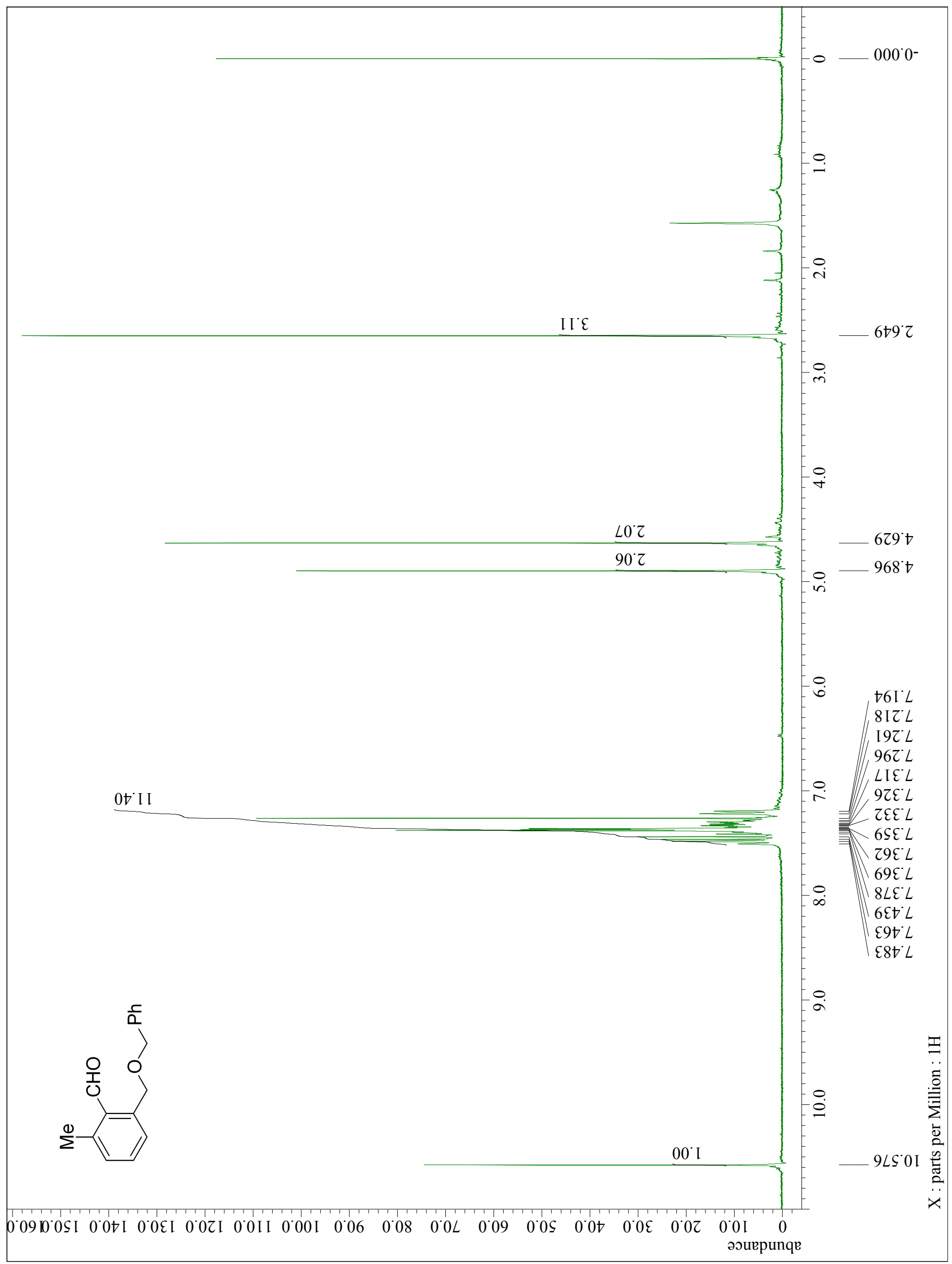


${ }^{13} \mathrm{C}$ NMR spectrum of $\mathbf{s 1 7}\left(\mathrm{CDCl}_{3}, 75 \mathrm{MHz}\right)$.

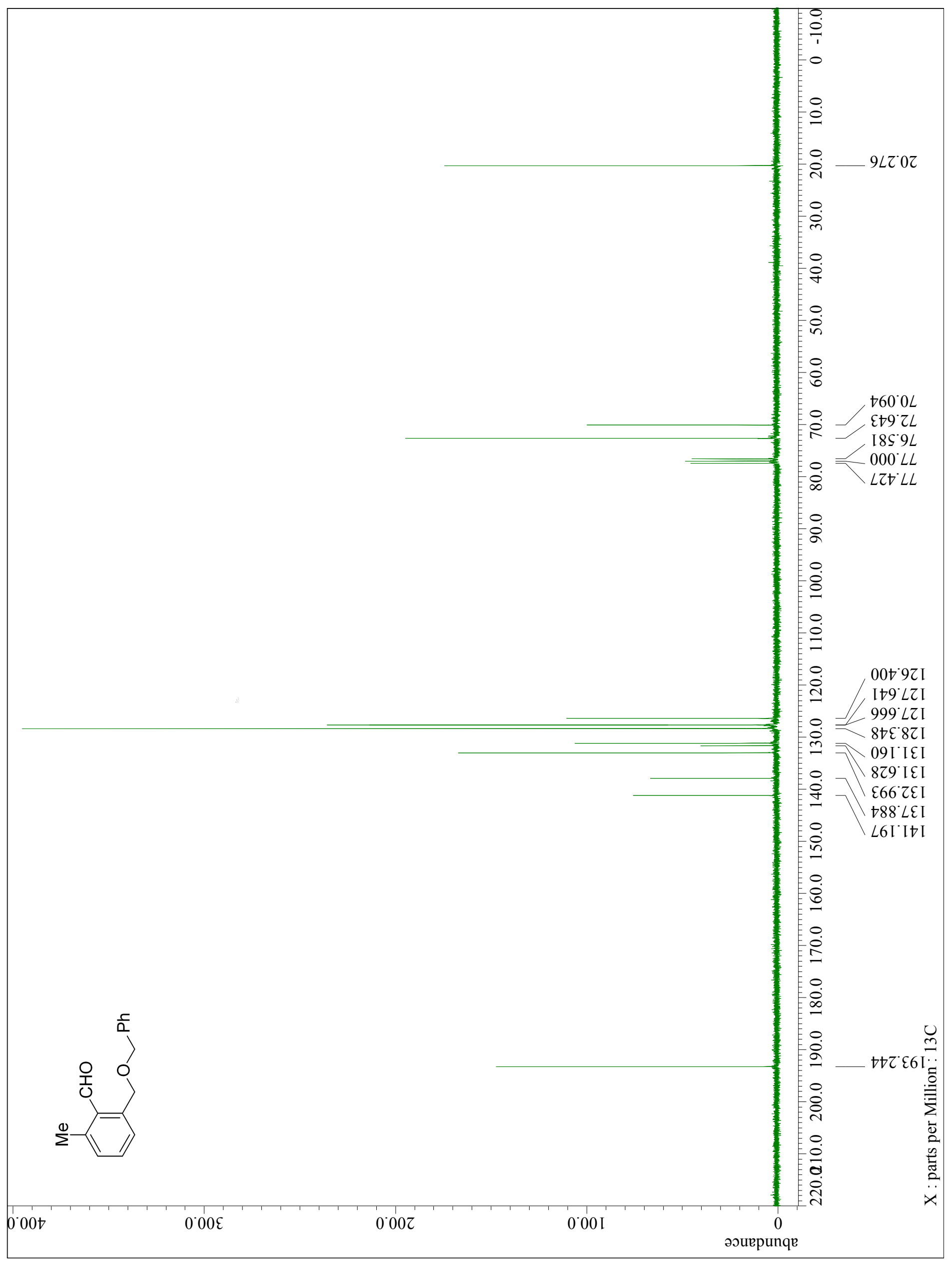


${ }^{1} \mathrm{H}$ NMR spectrum of $\mathbf{3 h}\left(\mathrm{CDCl}_{3}, 300 \mathrm{MHz}\right)$.

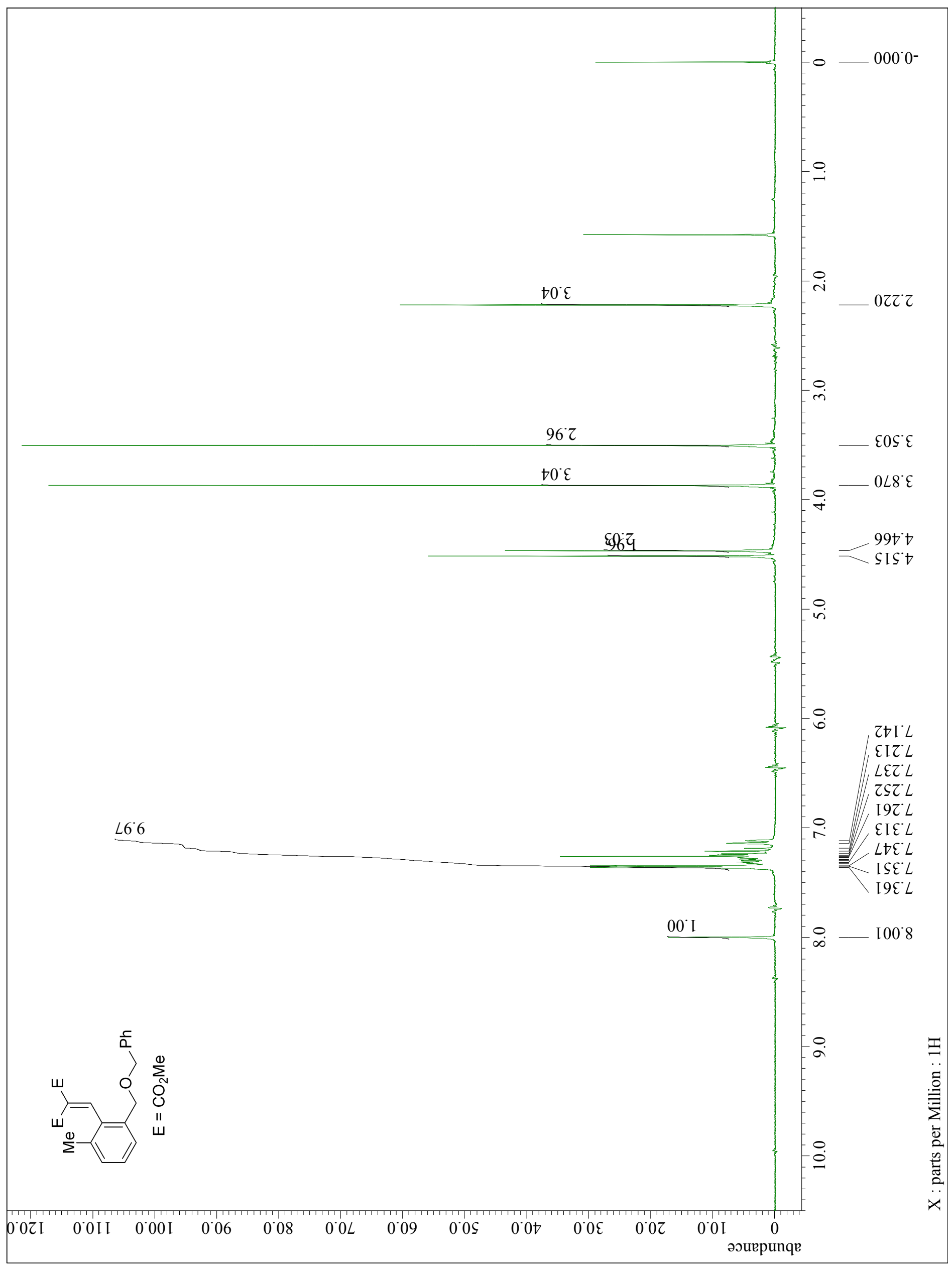




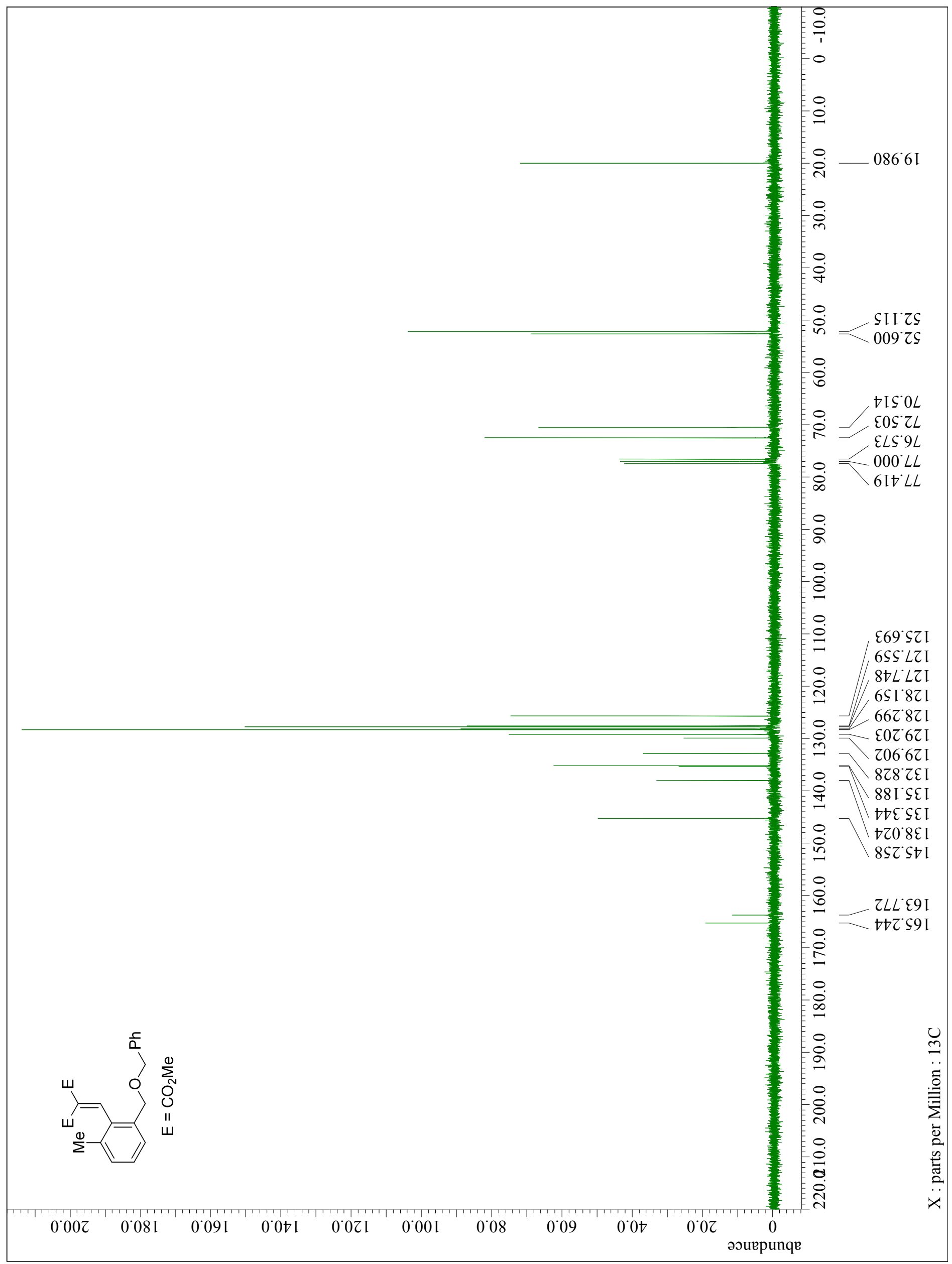


${ }^{1} \mathrm{H}$ NMR spectrum of $\mathbf{s 1 8}\left(\mathrm{CDCl}_{3}, 300 \mathrm{MHz}\right)$.

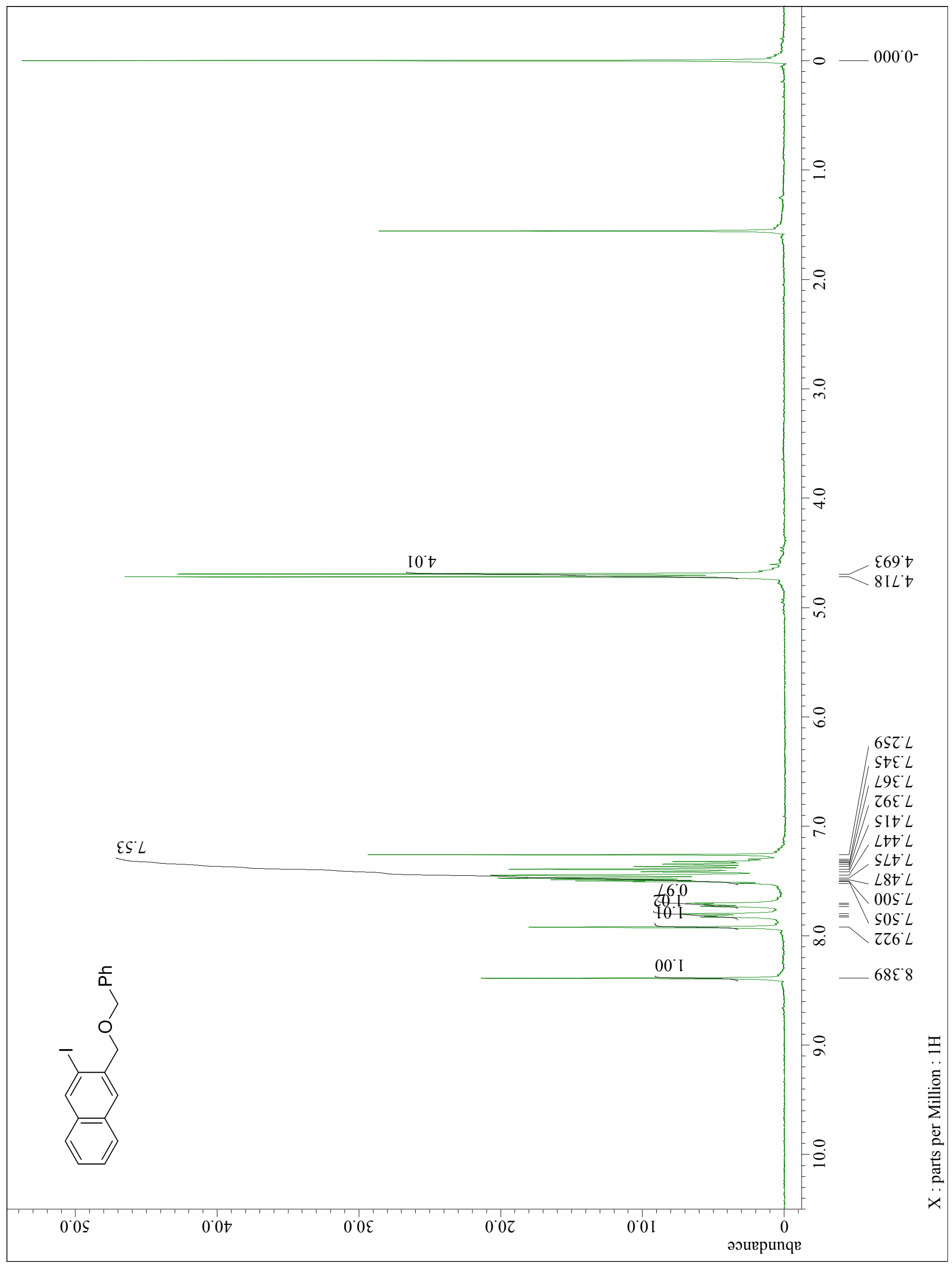


${ }^{13} \mathrm{C} \mathrm{NMR}$ spectrum of $\mathbf{s} 18\left(\mathrm{CDCl}_{3}, 75 \mathrm{MHz}\right)$.

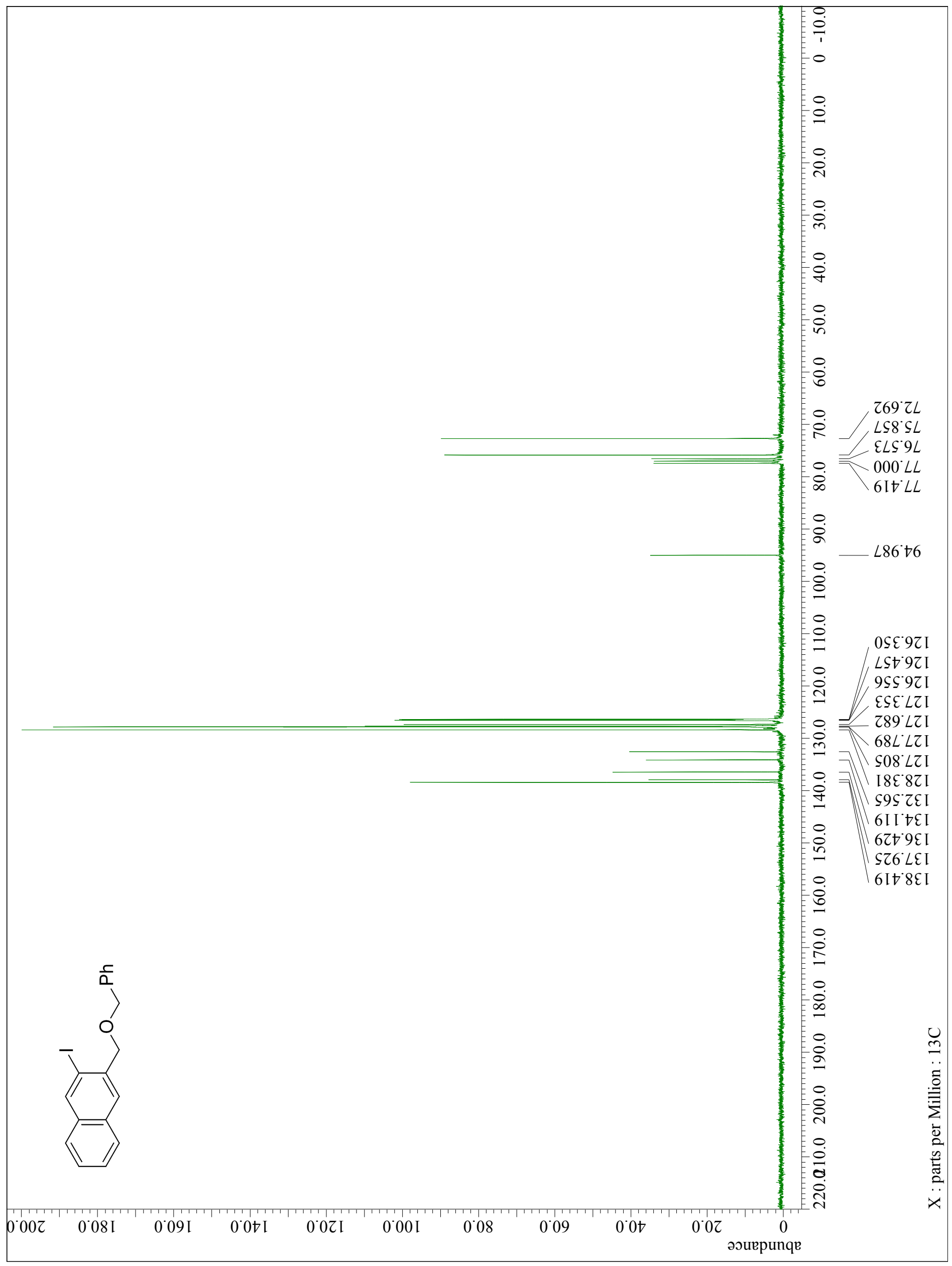


${ }^{1} \mathrm{H}$ NMR spectrum of $\mathbf{s 1 9}\left(\mathrm{CDCl}_{3}, 300 \mathrm{MHz}\right)$.

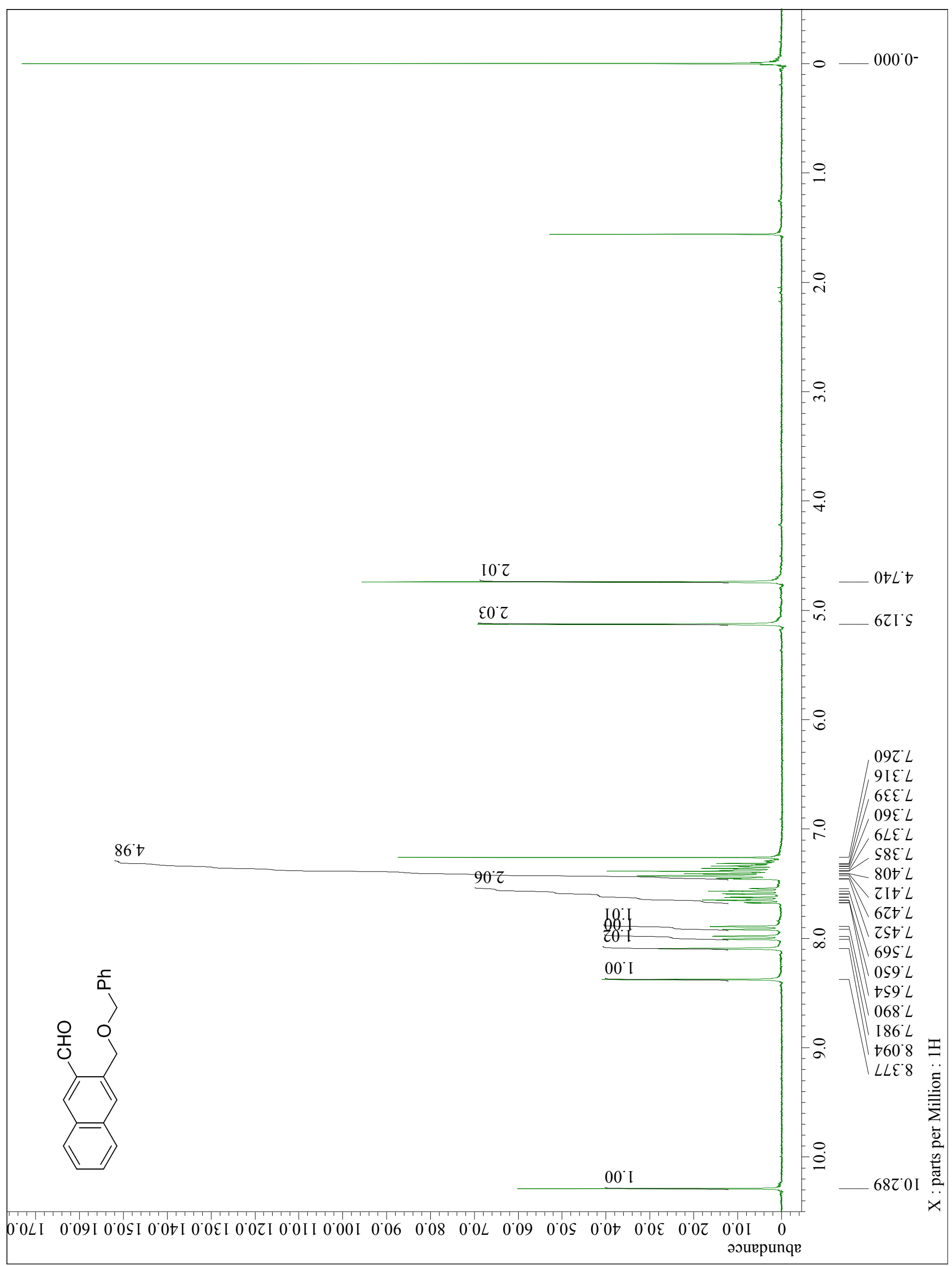


${ }^{13} \mathrm{C}$ NMR spectrum of $\mathbf{s 1 9}\left(\mathrm{CDCl}_{3}, 75 \mathrm{MHz}\right)$.

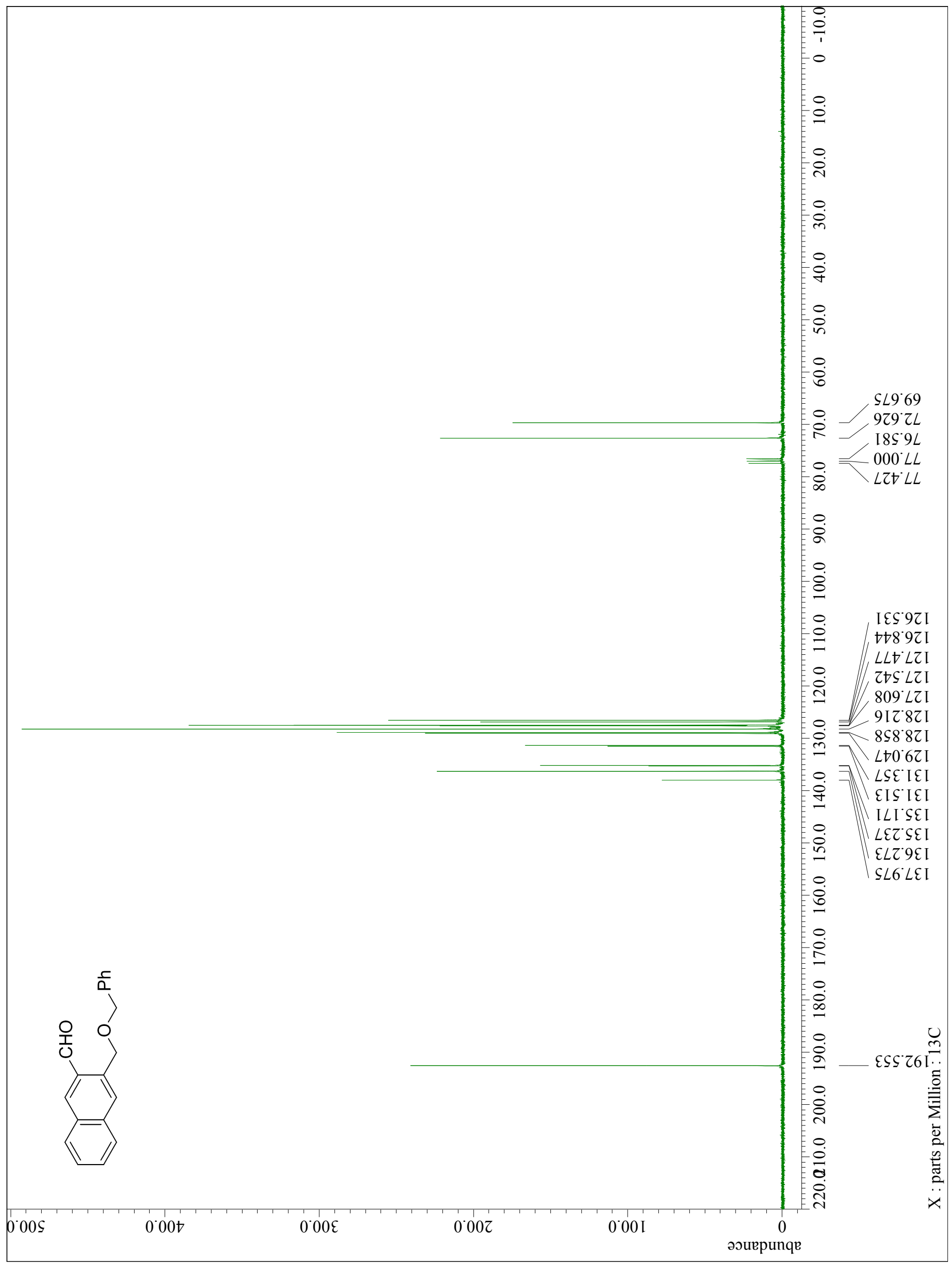


${ }^{1} \mathrm{H}$ NMR spectrum of $\mathbf{3 i}\left(\mathrm{CDCl}_{3}, 300 \mathrm{MHz}\right)$.

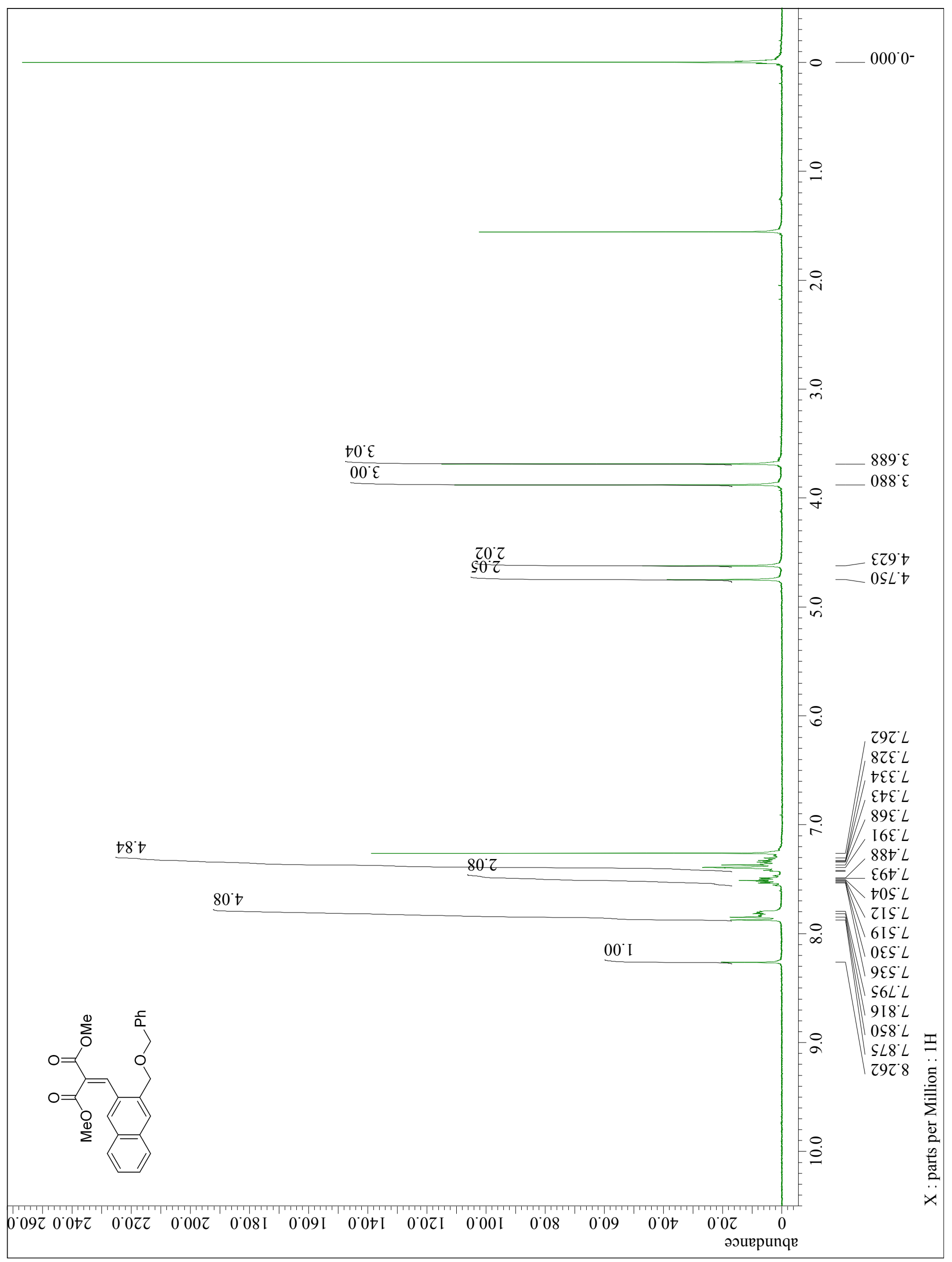


${ }^{13} \mathrm{C}$ NMR spectrum of $\mathbf{3 i}\left(\mathrm{CDCl}_{3}, 75 \mathrm{MHz}\right)$.

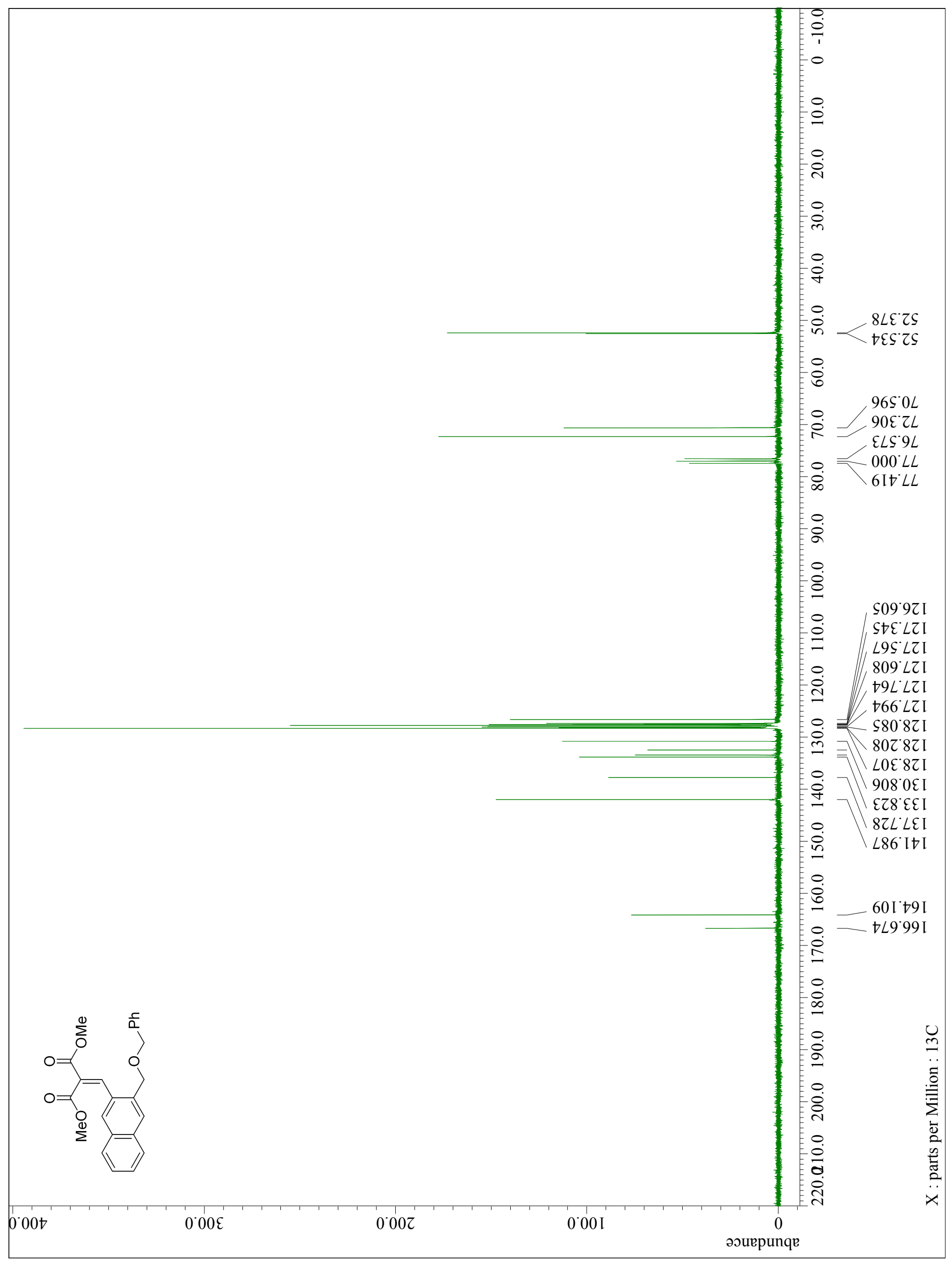


${ }^{1} \mathrm{H}$ NMR spectrum of $\mathbf{s 2 0}\left(\mathrm{CDCl}_{3}, 300 \mathrm{MHz}\right)$.

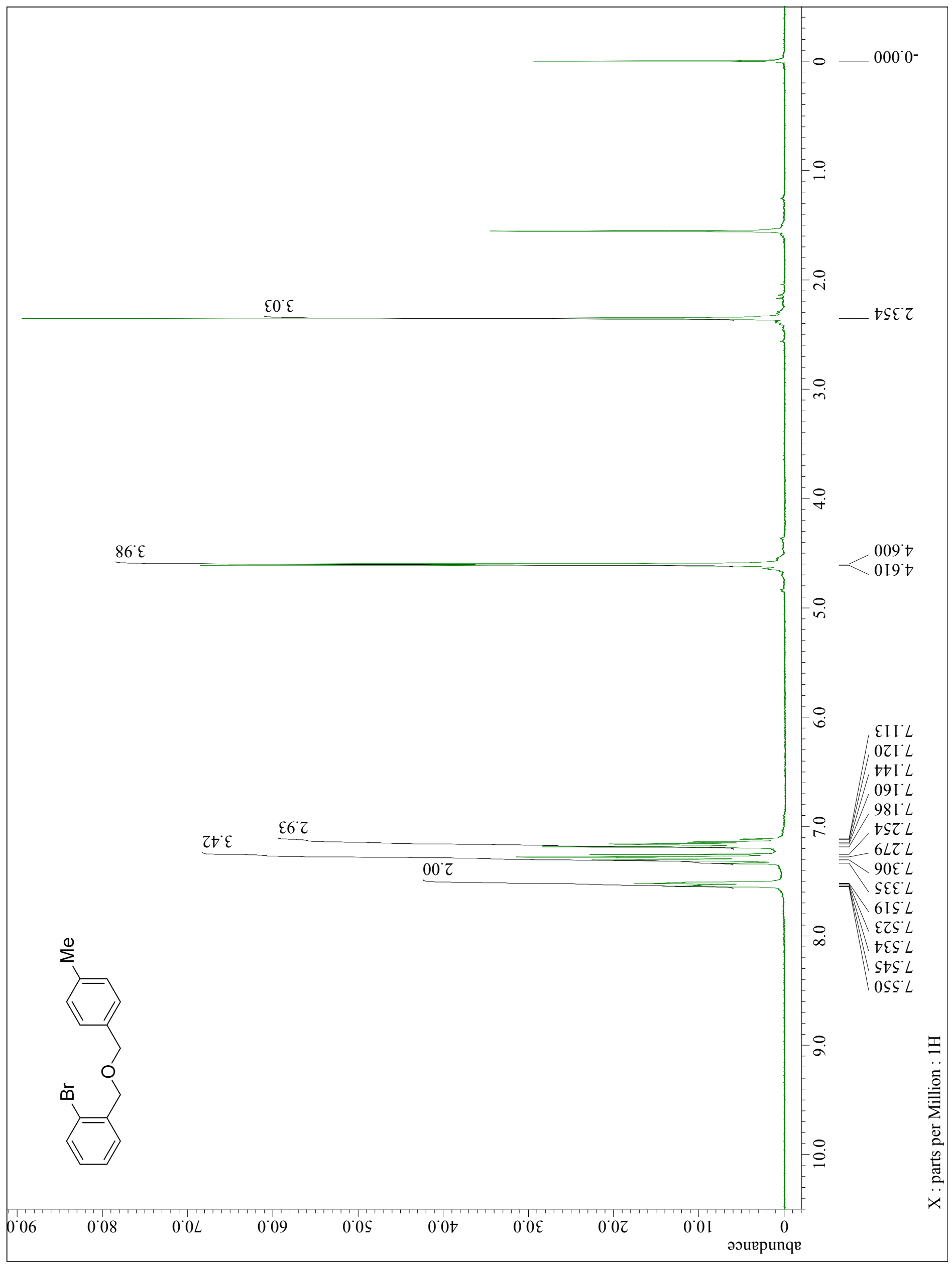


${ }^{13} \mathrm{C}$ NMR spectrum of $\mathbf{s 2 0}\left(\mathrm{CDCl}_{3}, 75 \mathrm{MHz}\right)$.

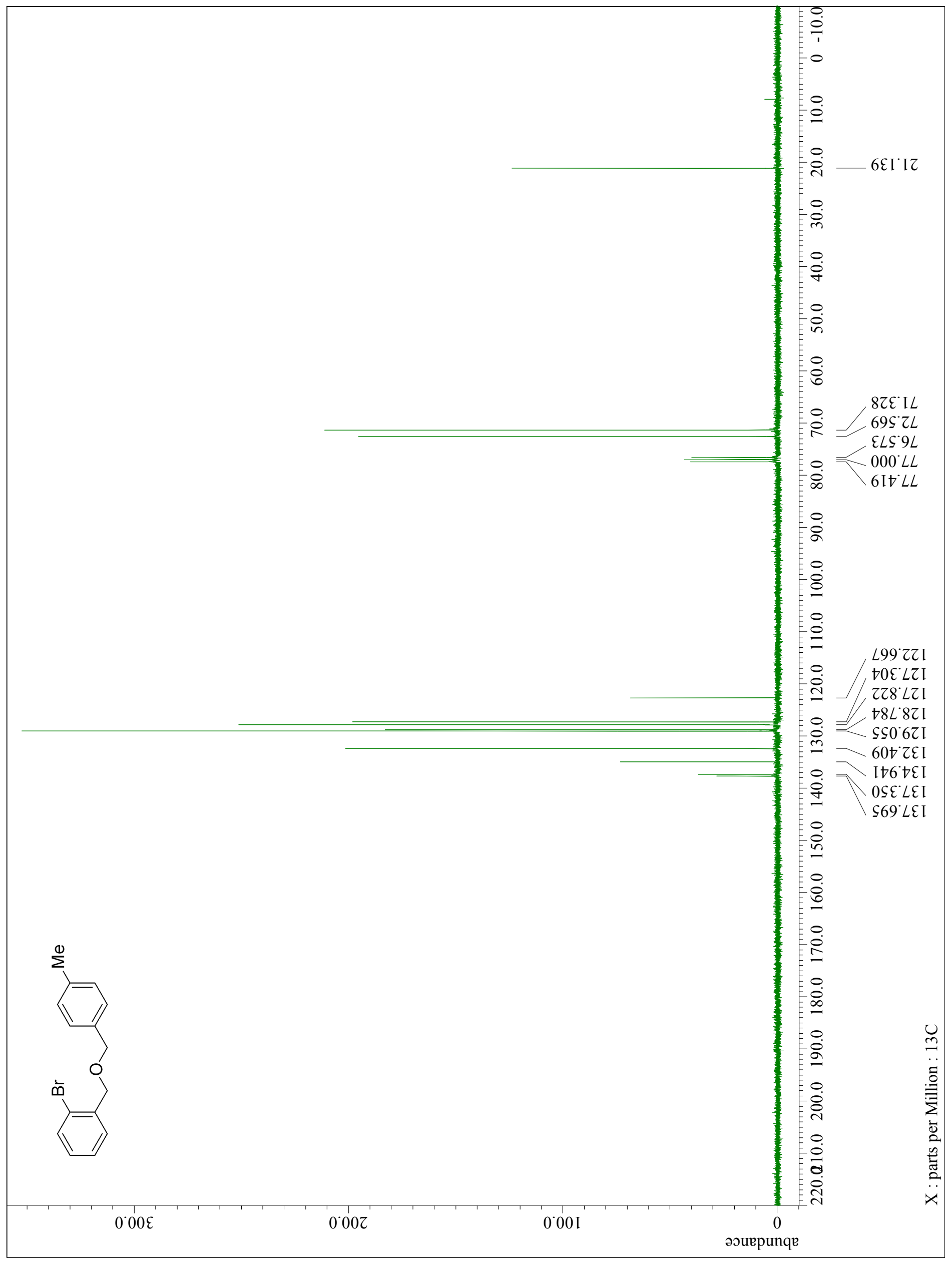


${ }^{1} \mathrm{H}$ NMR spectrum of $\mathbf{s} 21\left(\mathrm{CDCl}_{3}, 300 \mathrm{MHz}\right)$.

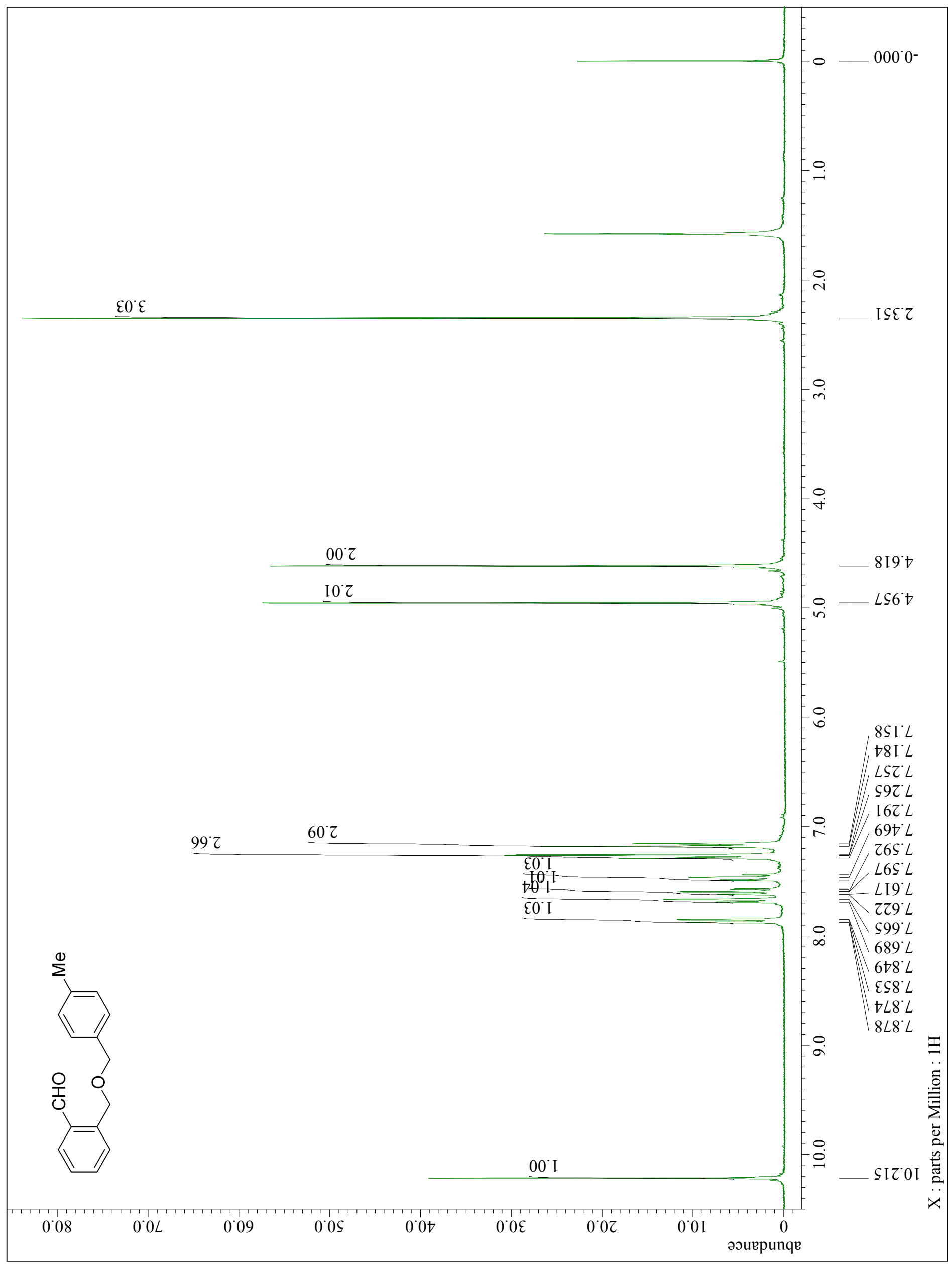




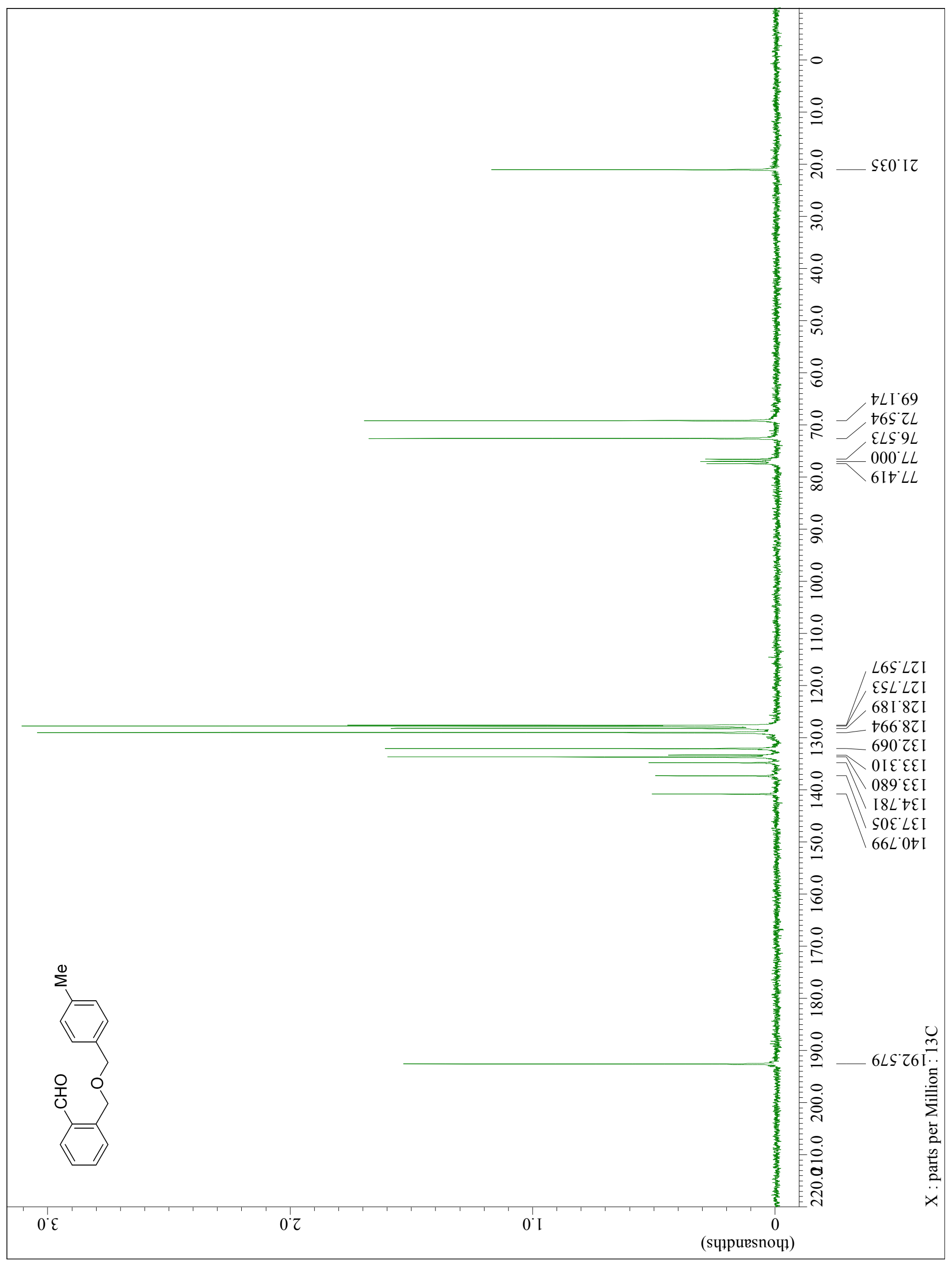


${ }^{1} \mathrm{H}$ NMR spectrum of $\mathbf{3 j}\left(\mathrm{CDCl}_{3}, 300 \mathrm{MHz}\right)$.

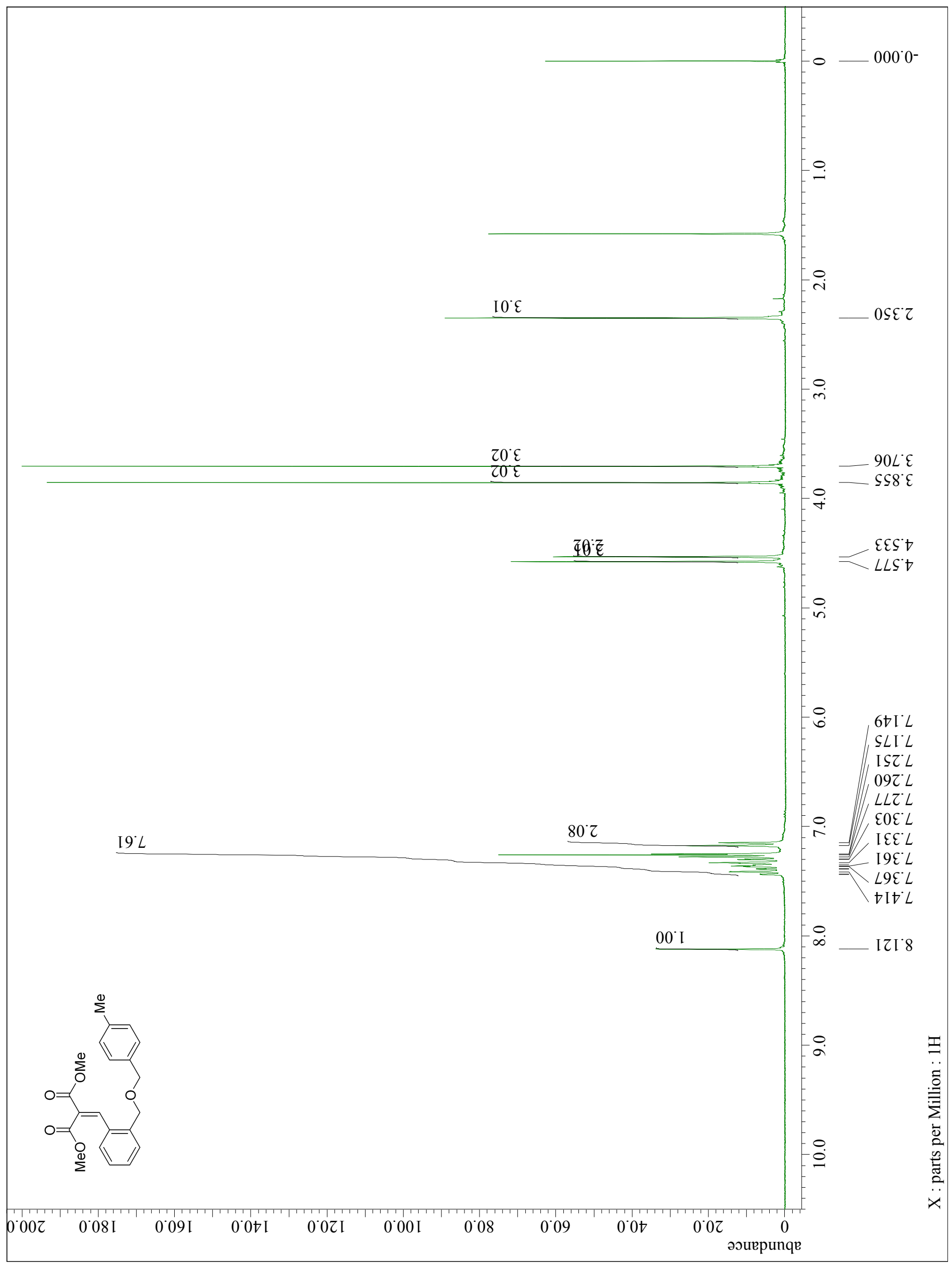


${ }^{13} \mathrm{C} \mathrm{NMR}$ spectrum of $\mathbf{3 j}\left(\mathrm{CDCl}_{3}, 75 \mathrm{MHz}\right)$.

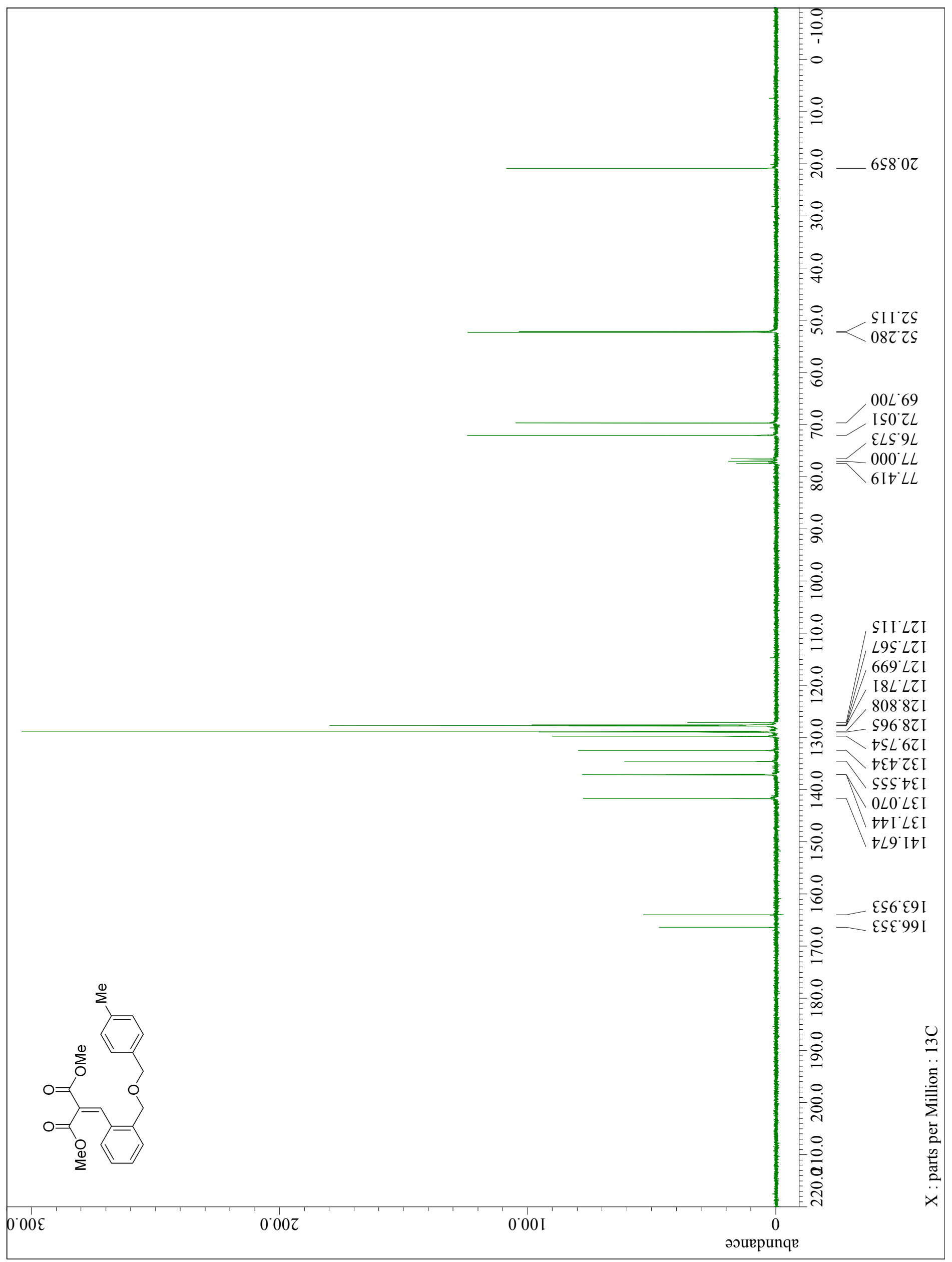


${ }^{1} \mathrm{H}$ NMR spectrum of $\mathbf{s 2 2}\left(\mathrm{CDCl}_{3}, 300 \mathrm{MHz}\right)$.

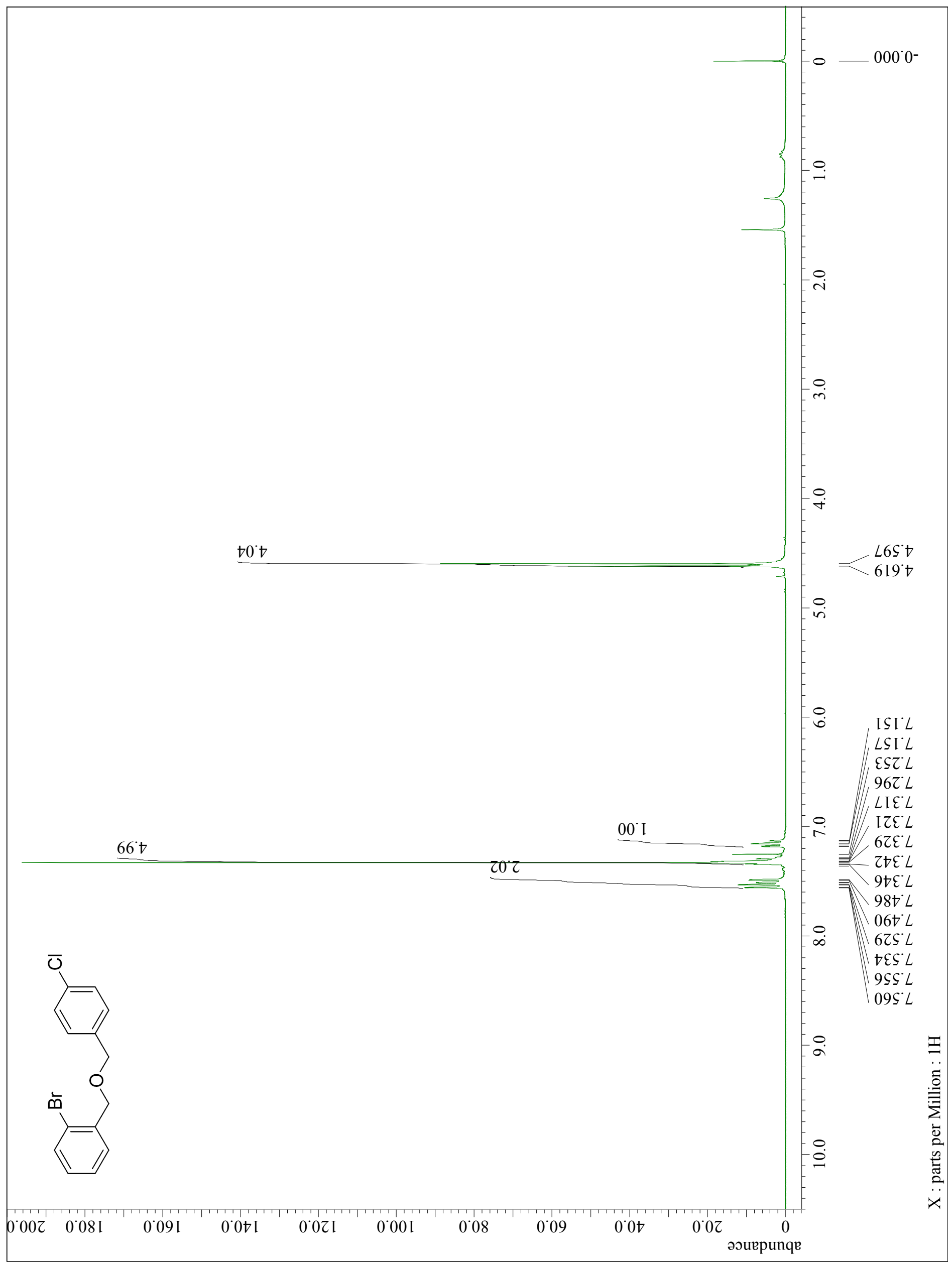


${ }^{13} \mathrm{C}$ NMR spectrum of $\mathbf{s} 22\left(\mathrm{CDCl}_{3}, 75 \mathrm{MHz}\right)$.

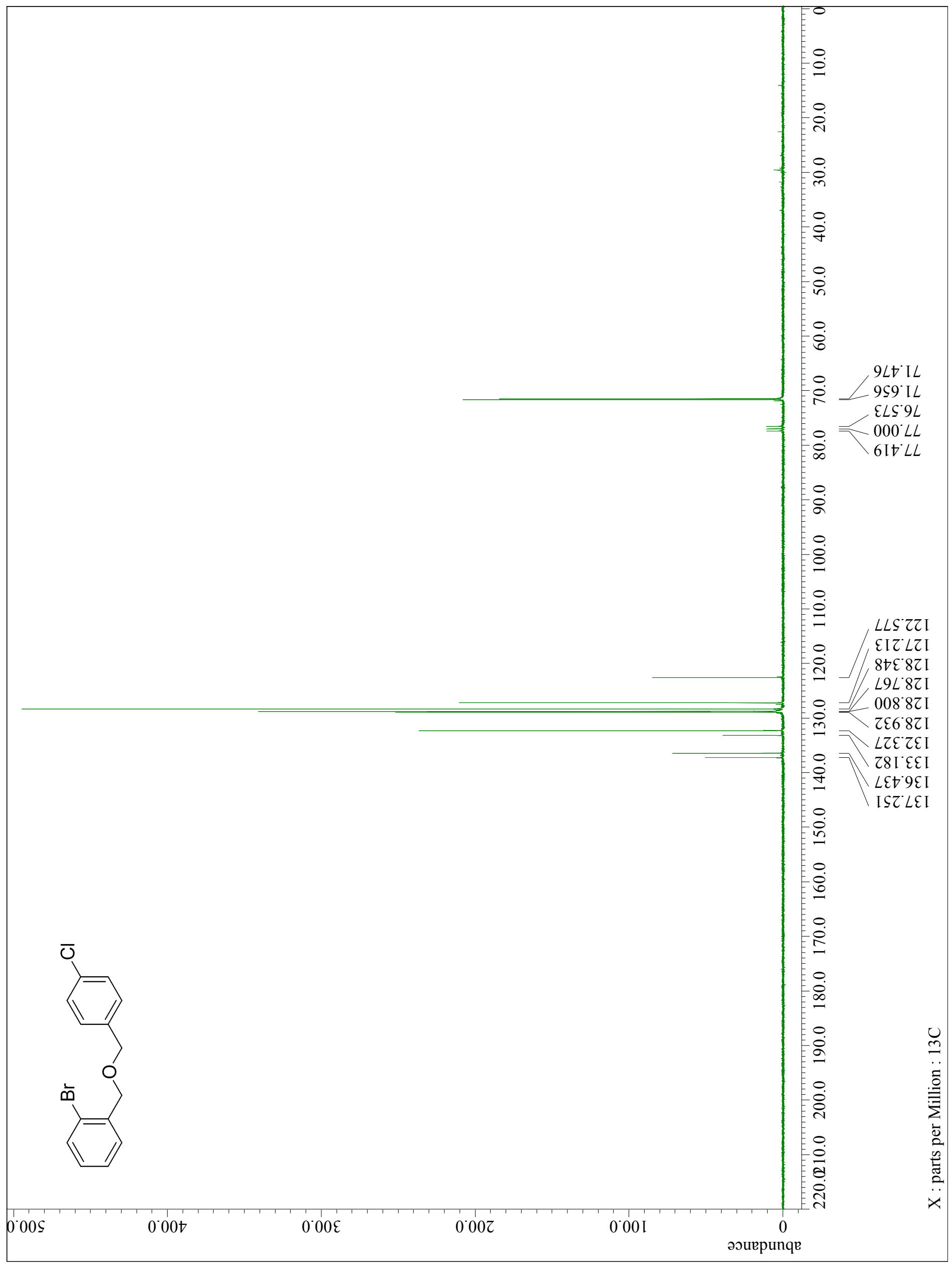


${ }^{1} \mathrm{H}$ NMR spectrum of $\mathbf{s 2 3}\left(\mathrm{CDCl}_{3}, 300 \mathrm{MHz}\right)$.

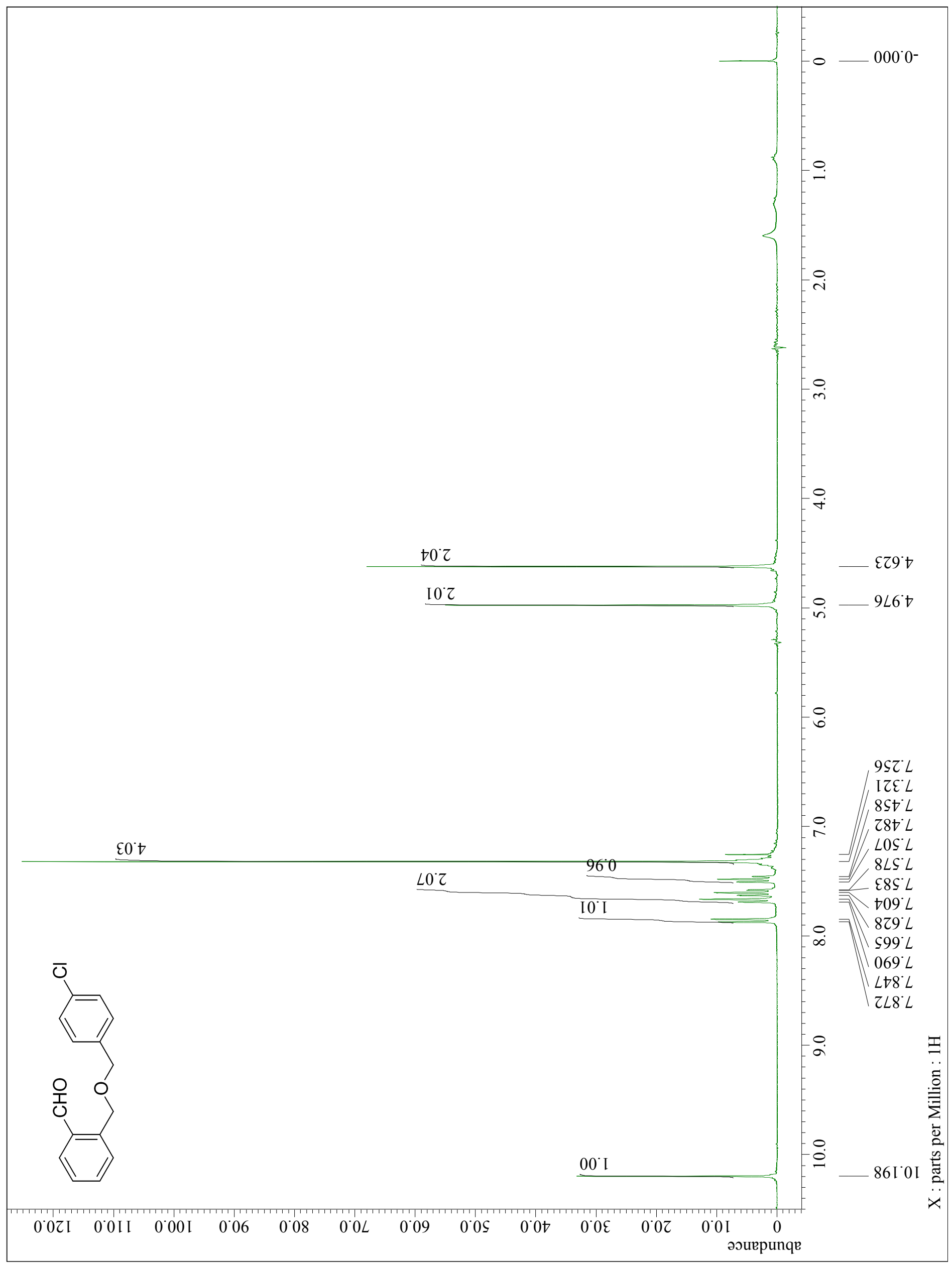




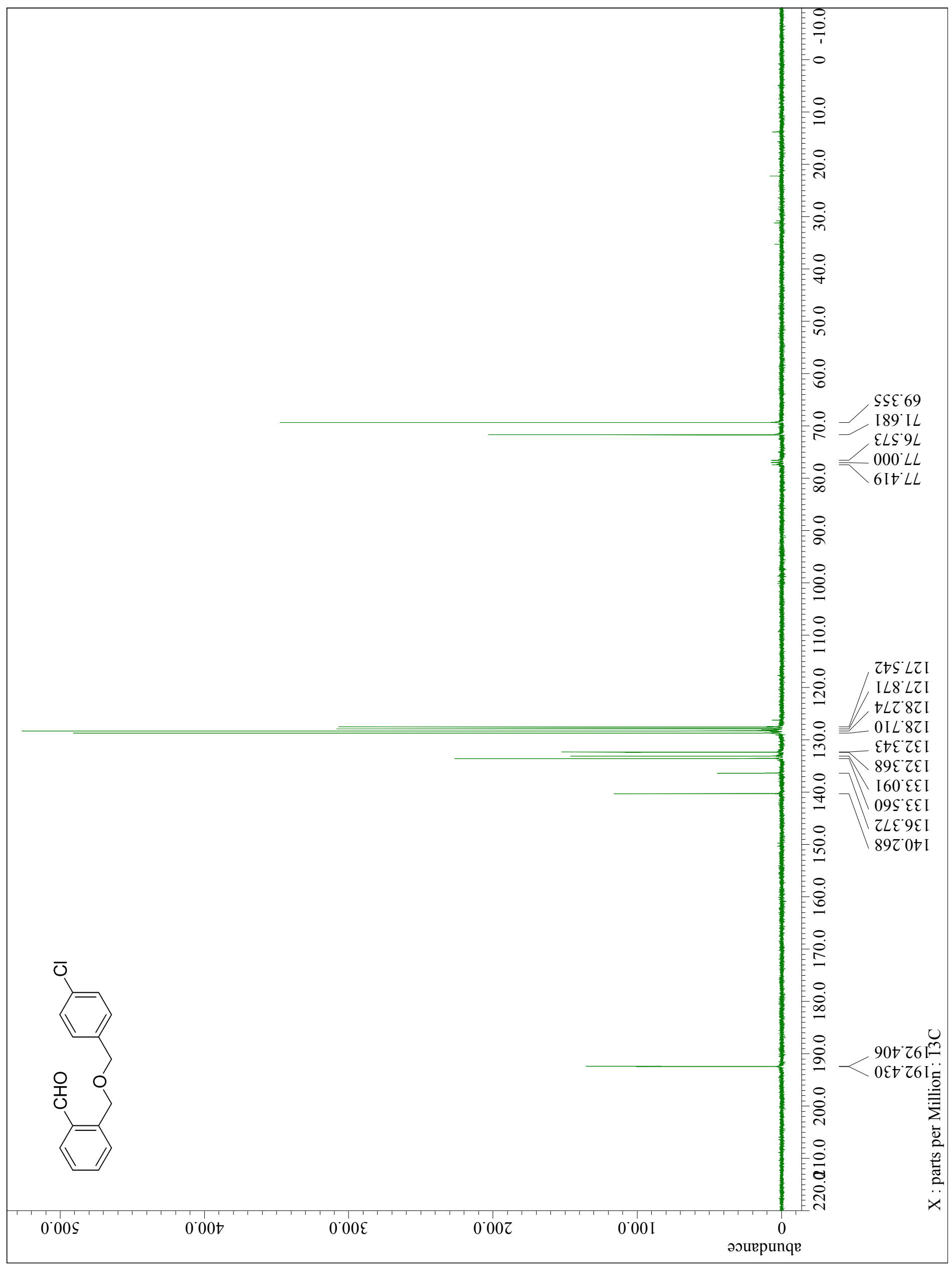


${ }^{1} \mathrm{H}$ NMR spectrum of $\mathbf{3 k}\left(\mathrm{CDCl}_{3}, 300 \mathrm{MHz}\right)$.

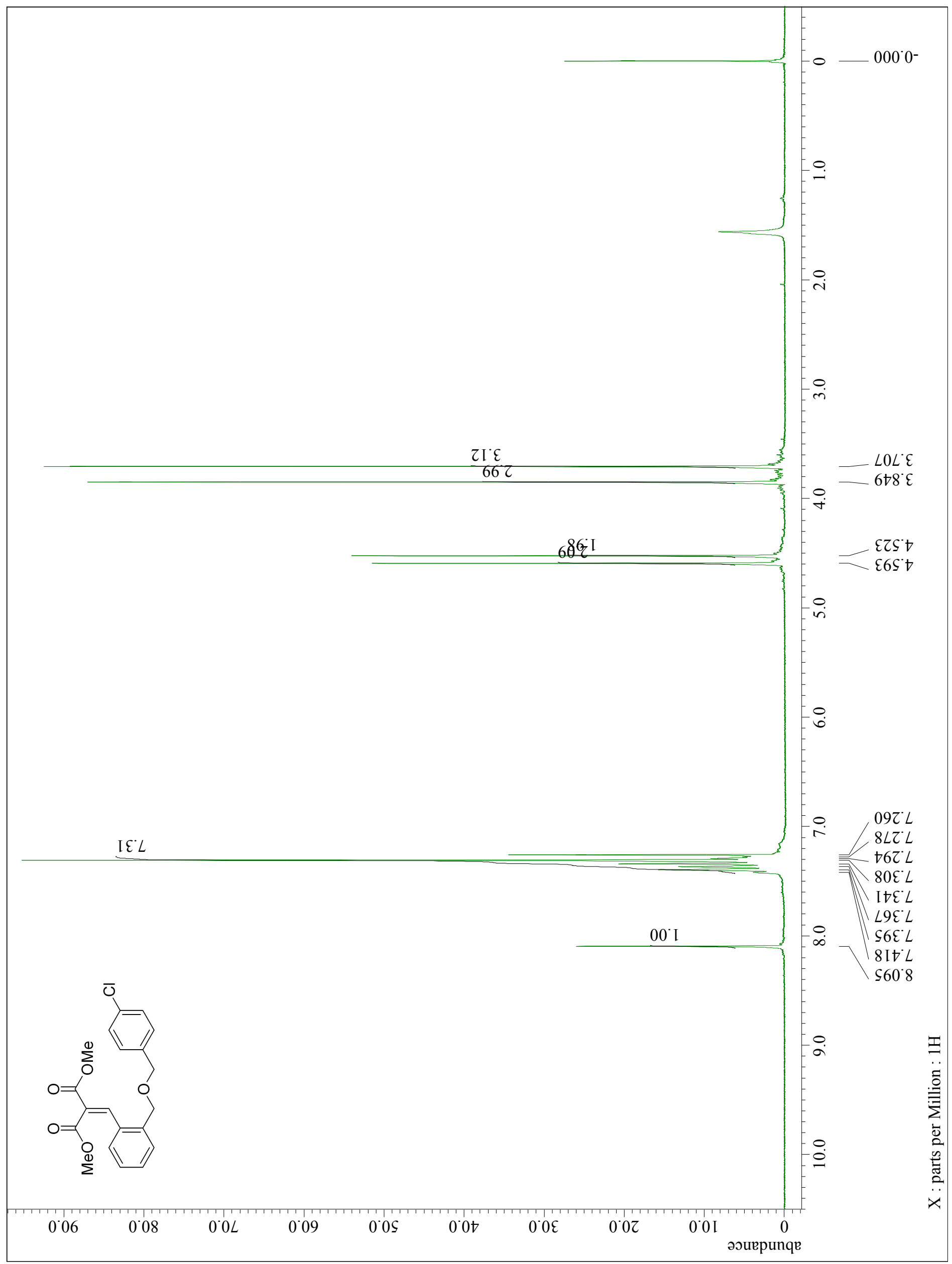


${ }^{13} \mathrm{C}$ NMR spectrum of $\mathbf{3 k}\left(\mathrm{CDCl}_{3}, 75 \mathrm{MHz}\right)$.

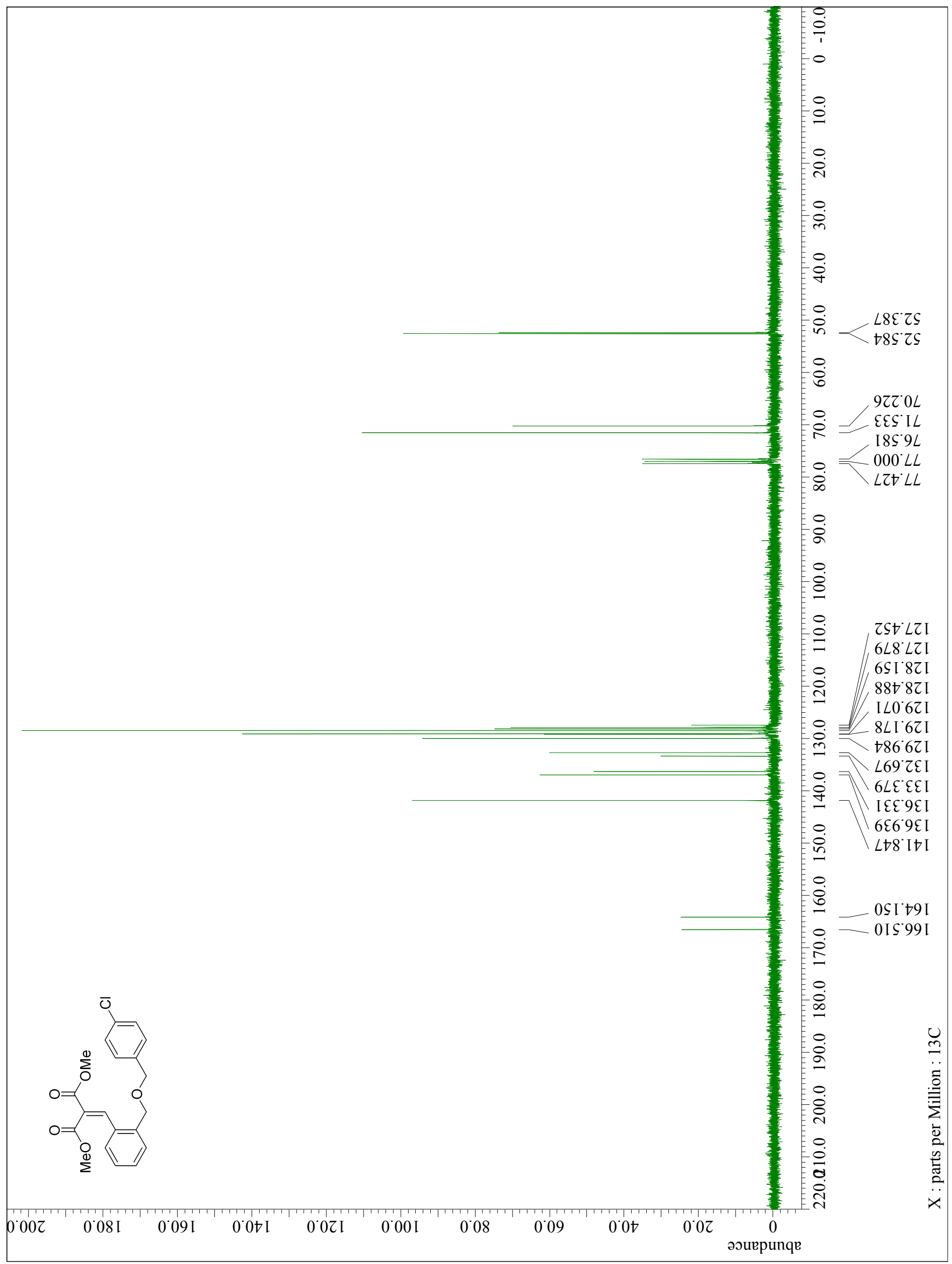


${ }^{1} \mathrm{H}$ NMR spectrum of $\mathbf{s 2 4}\left(\mathrm{CDCl}_{3}, 300 \mathrm{MHz}\right)$.

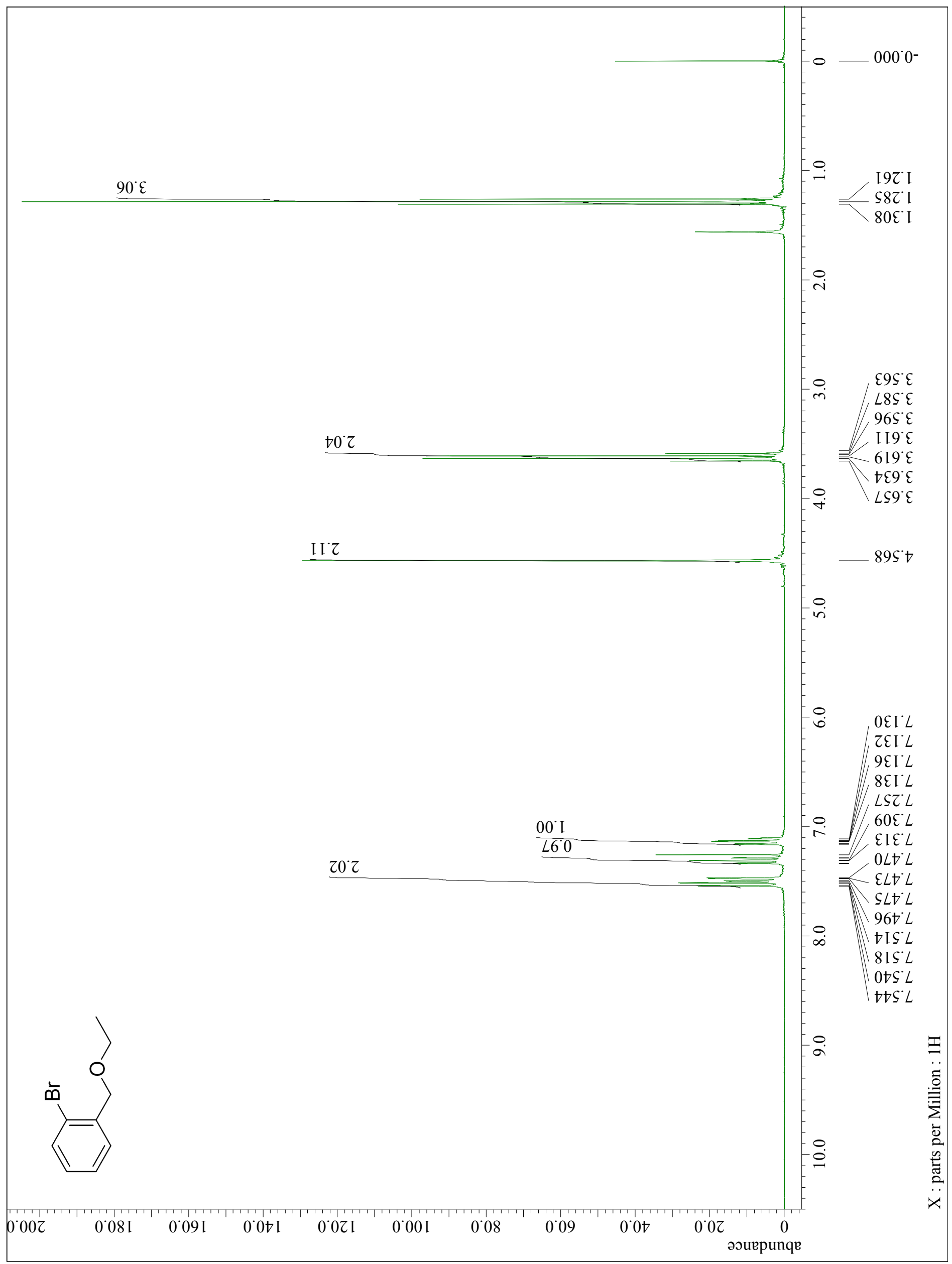


${ }^{13} \mathrm{C}$ NMR spectrum of $\mathbf{s} 24\left(\mathrm{CDCl}_{3}, 75 \mathrm{MHz}\right)$.

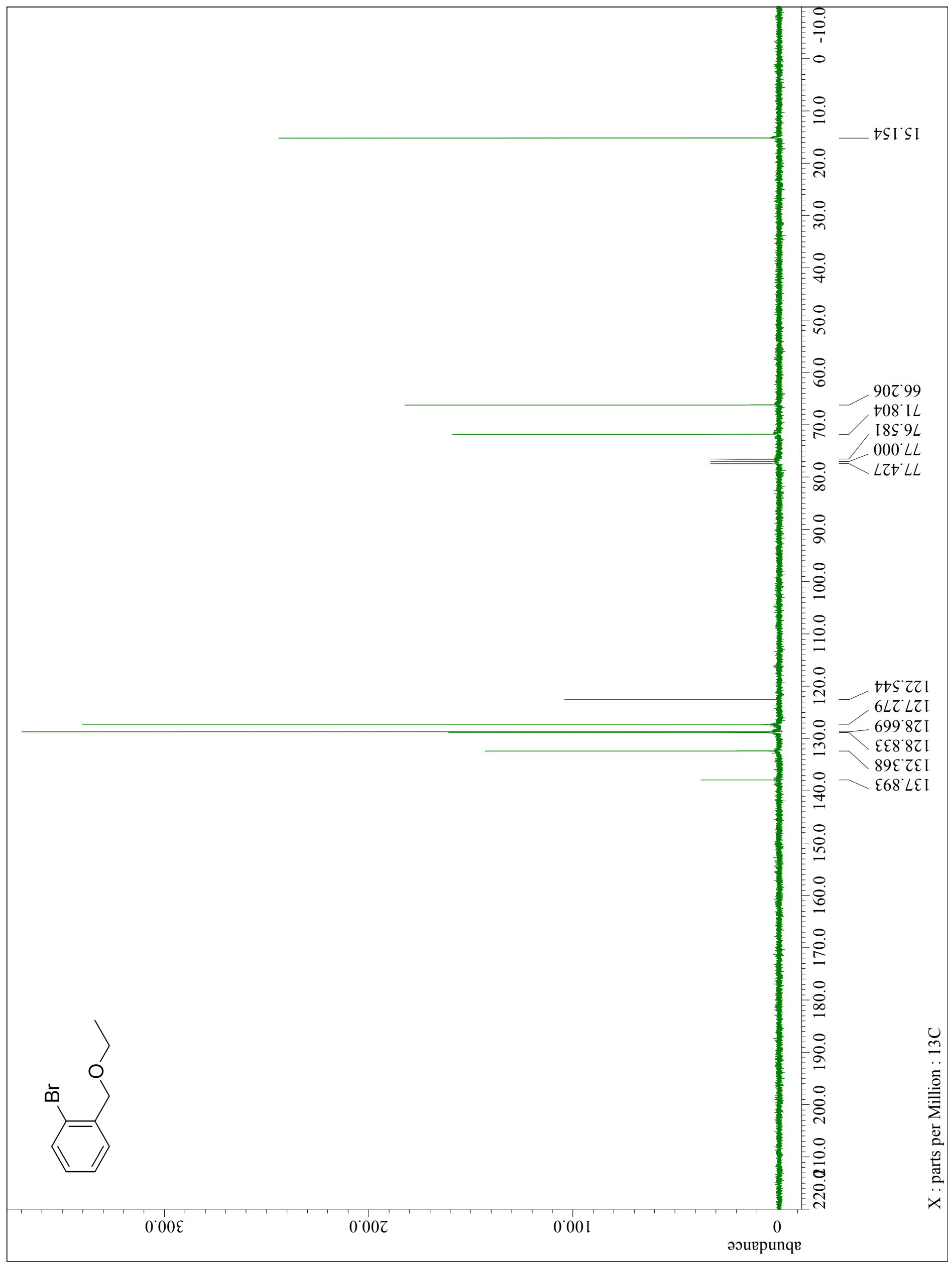


${ }^{13} \mathrm{C}$ NMR spectrum of $\mathbf{s} 25\left(\mathrm{CDCl}_{3}, 75 \mathrm{MHz}\right)$.

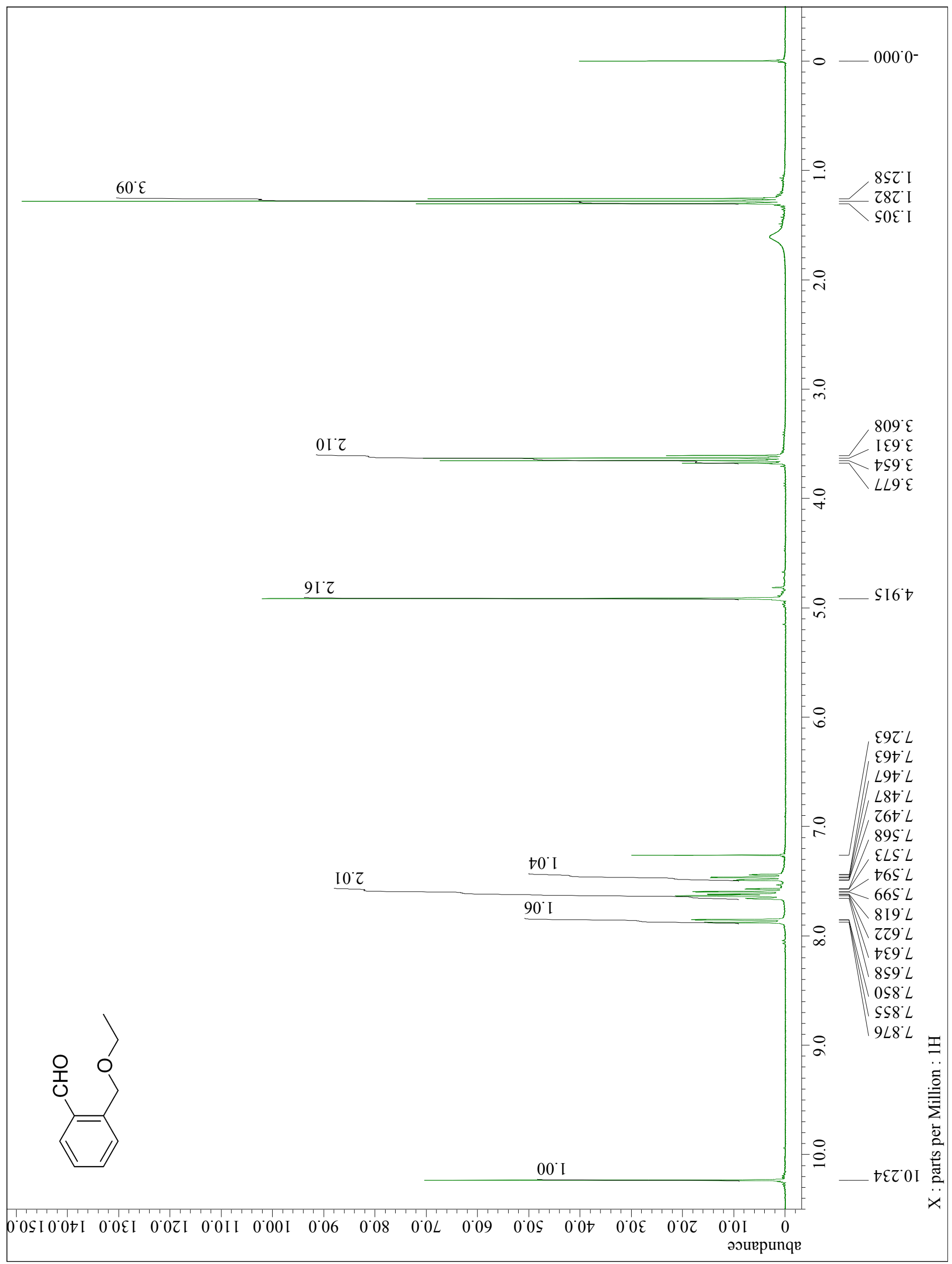


${ }^{13} \mathrm{C} \mathrm{NMR}$ spectrum of $\mathbf{s 2 5}\left(\mathrm{CDCl}_{3}, 75 \mathrm{MHz}\right)$.

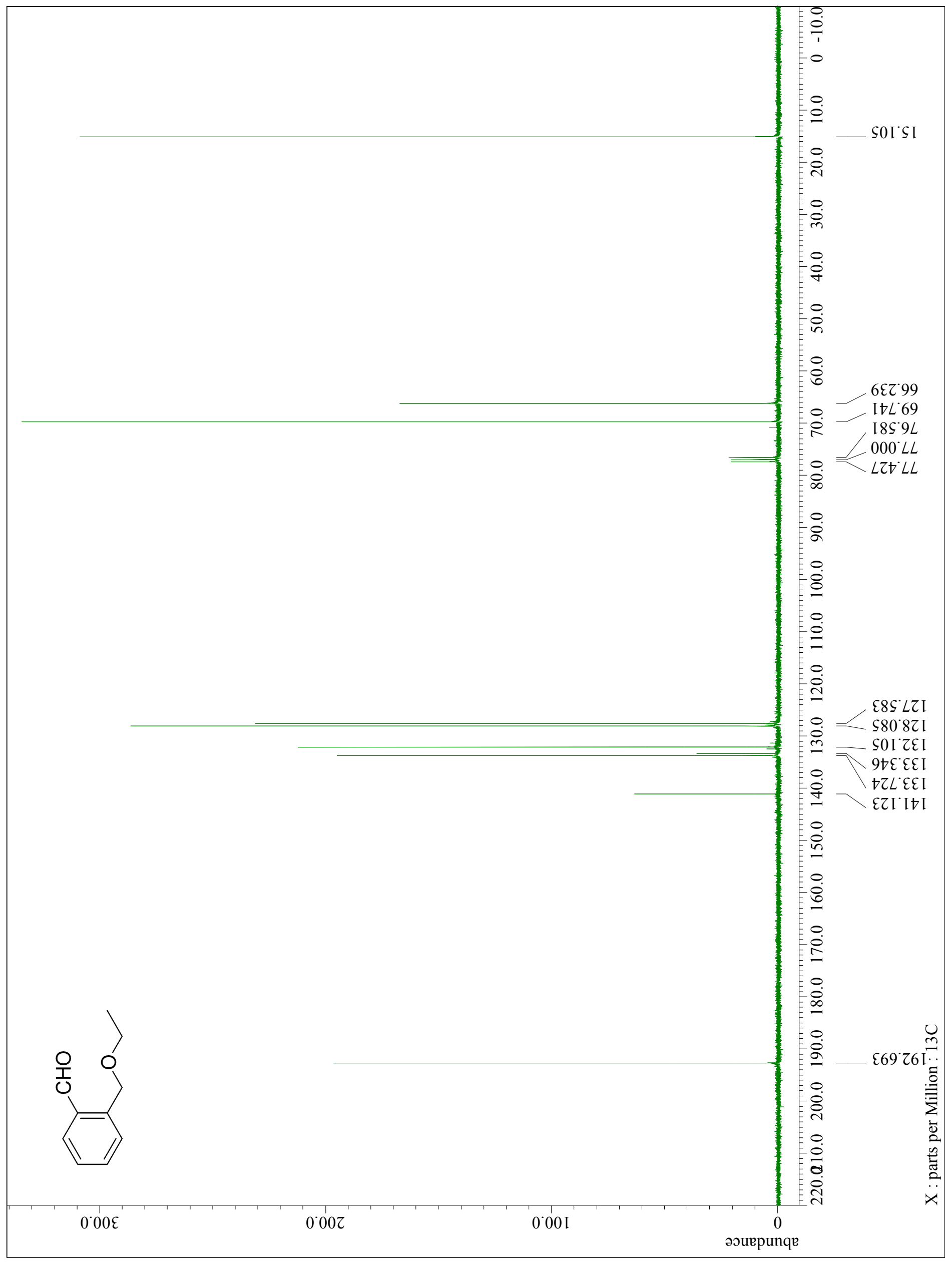


${ }^{1} \mathrm{H}$ NMR spectrum of $\mathbf{3 l}\left(\mathrm{CDCl}_{3}, 300 \mathrm{MHz}\right)$.

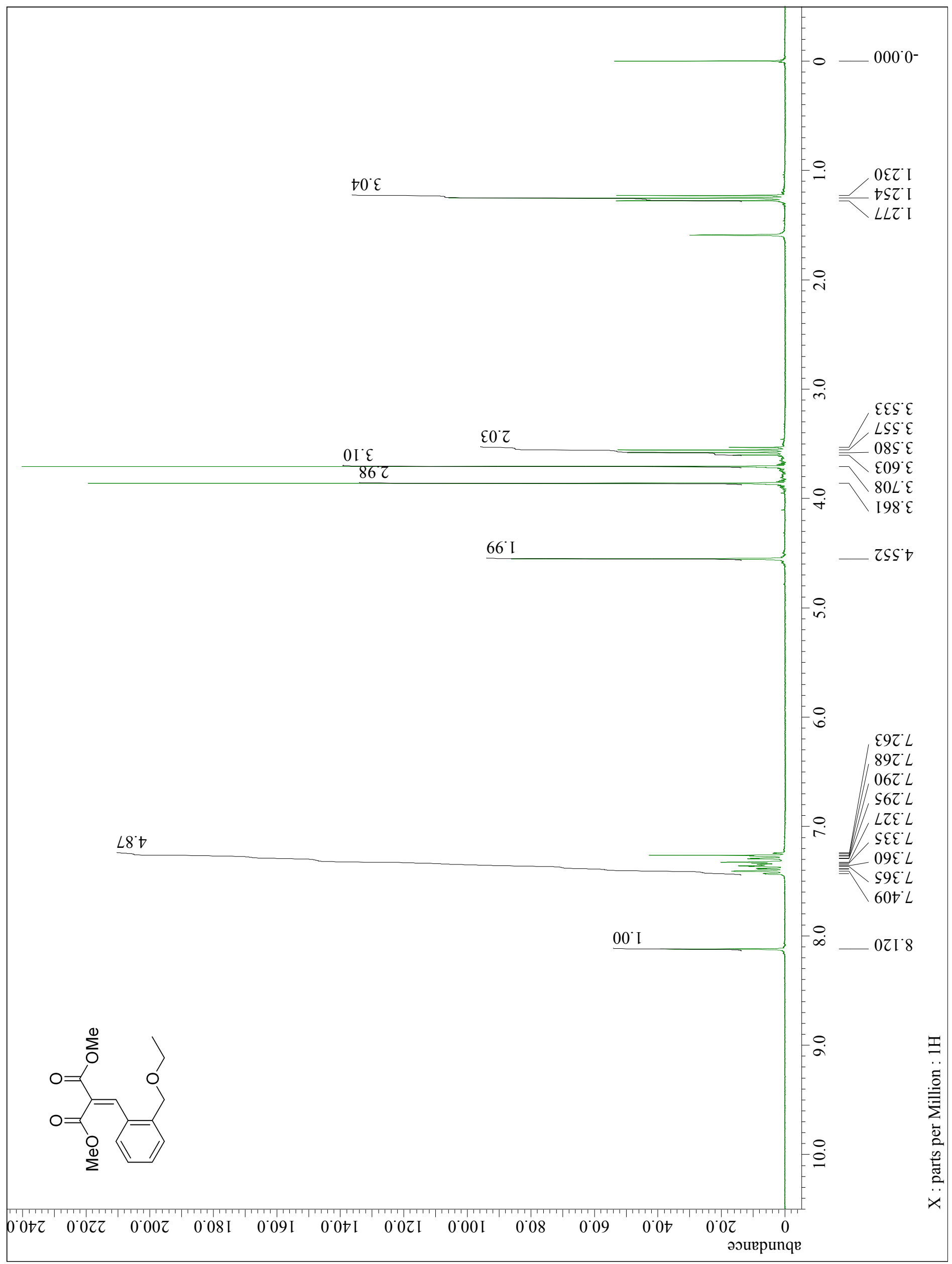


${ }^{13} \mathrm{C}$ NMR spectrum of $\mathbf{3 l}\left(\mathrm{CDCl}_{3}, 75 \mathrm{MHz}\right)$.

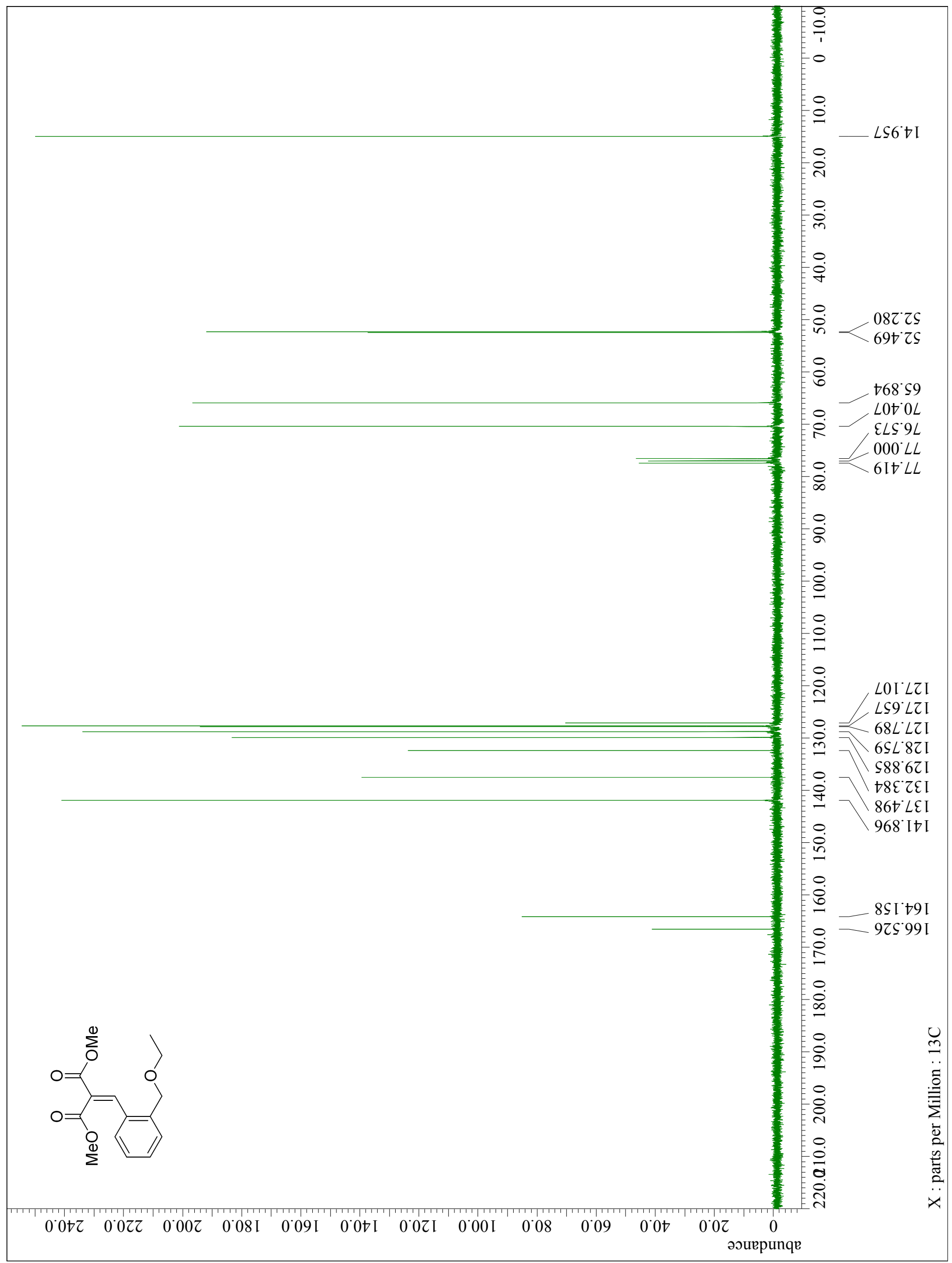


${ }^{1} \mathrm{H}$ NMR spectrum of $\mathbf{5 a}\left(\mathrm{CDCl}_{3}, 300 \mathrm{MHz}\right)$.

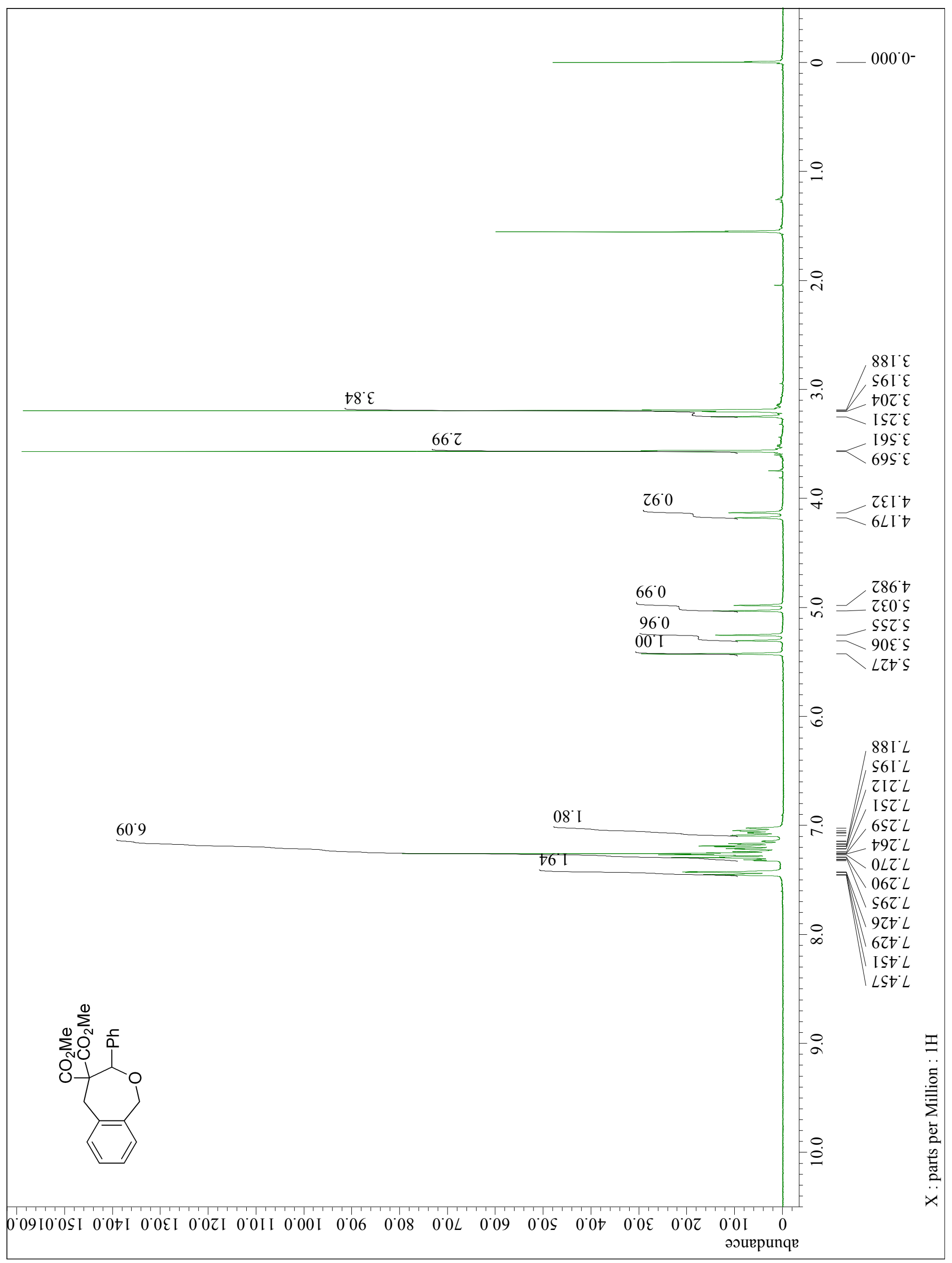




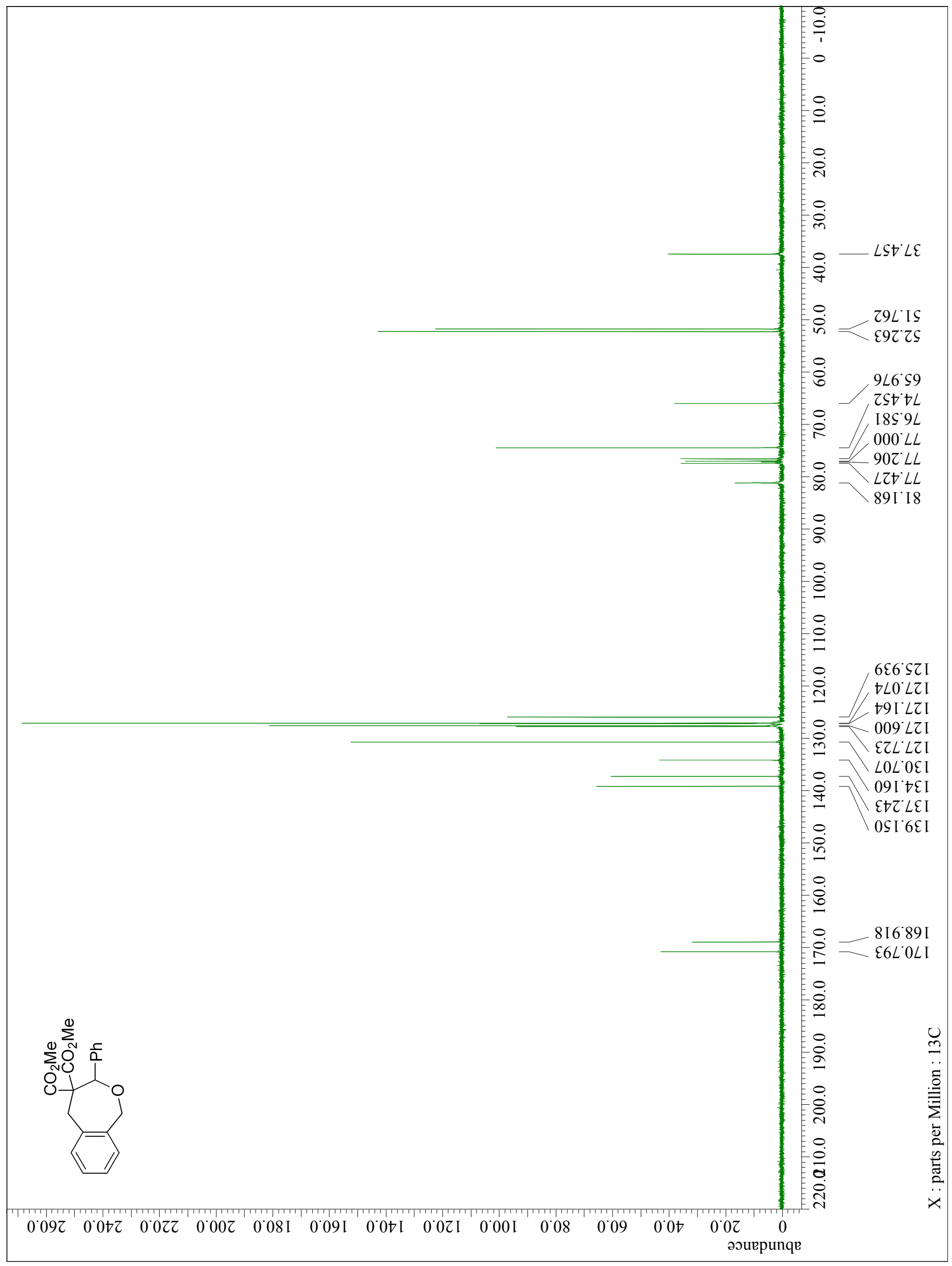


${ }^{1} \mathrm{H}$ NMR spectrum of $\mathbf{5 a}-\mathbf{d} \mathbf{2}\left(\mathrm{CDCl}_{3}, 300 \mathrm{MHz}\right)$.

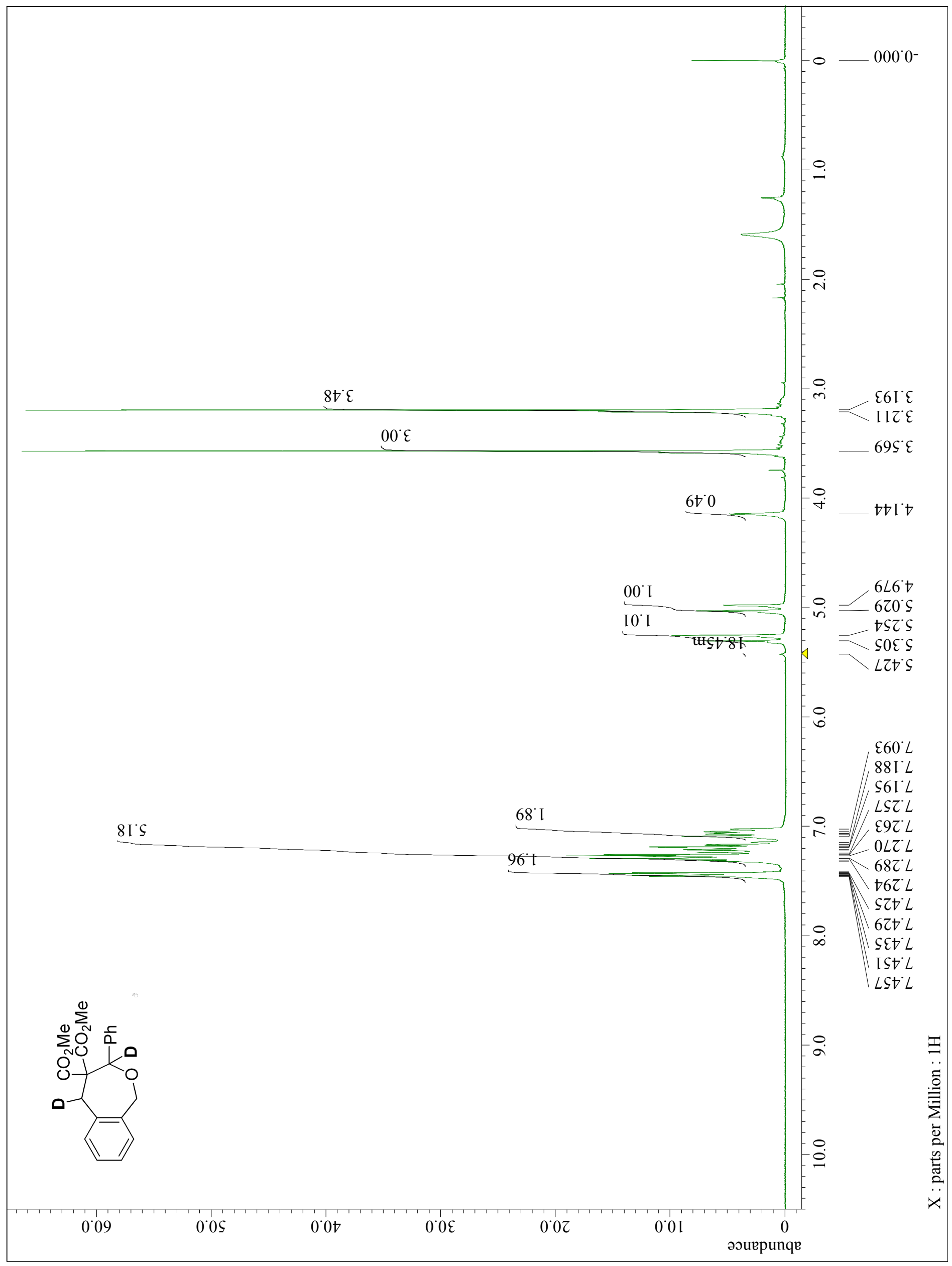


${ }^{1} \mathrm{H}$ NMR spectrum of d2-5a $\left(\mathrm{CDCl}_{3}, 300 \mathrm{MHz}\right)$.

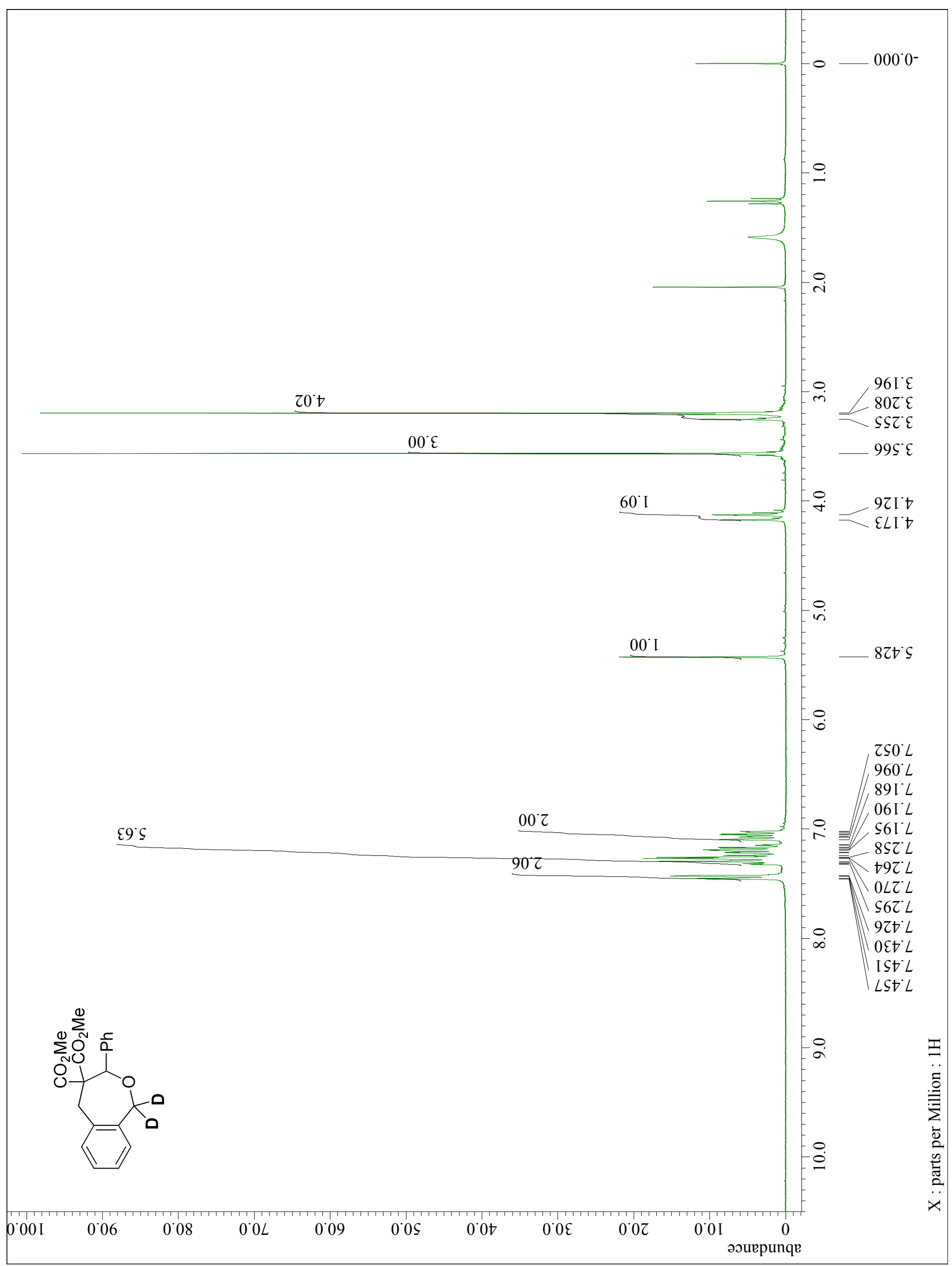


${ }^{1} \mathrm{H}$ NMR spectrum of $\mathbf{6 a}\left(\mathrm{CDCl}_{3}, 300 \mathrm{MHz}\right)$.

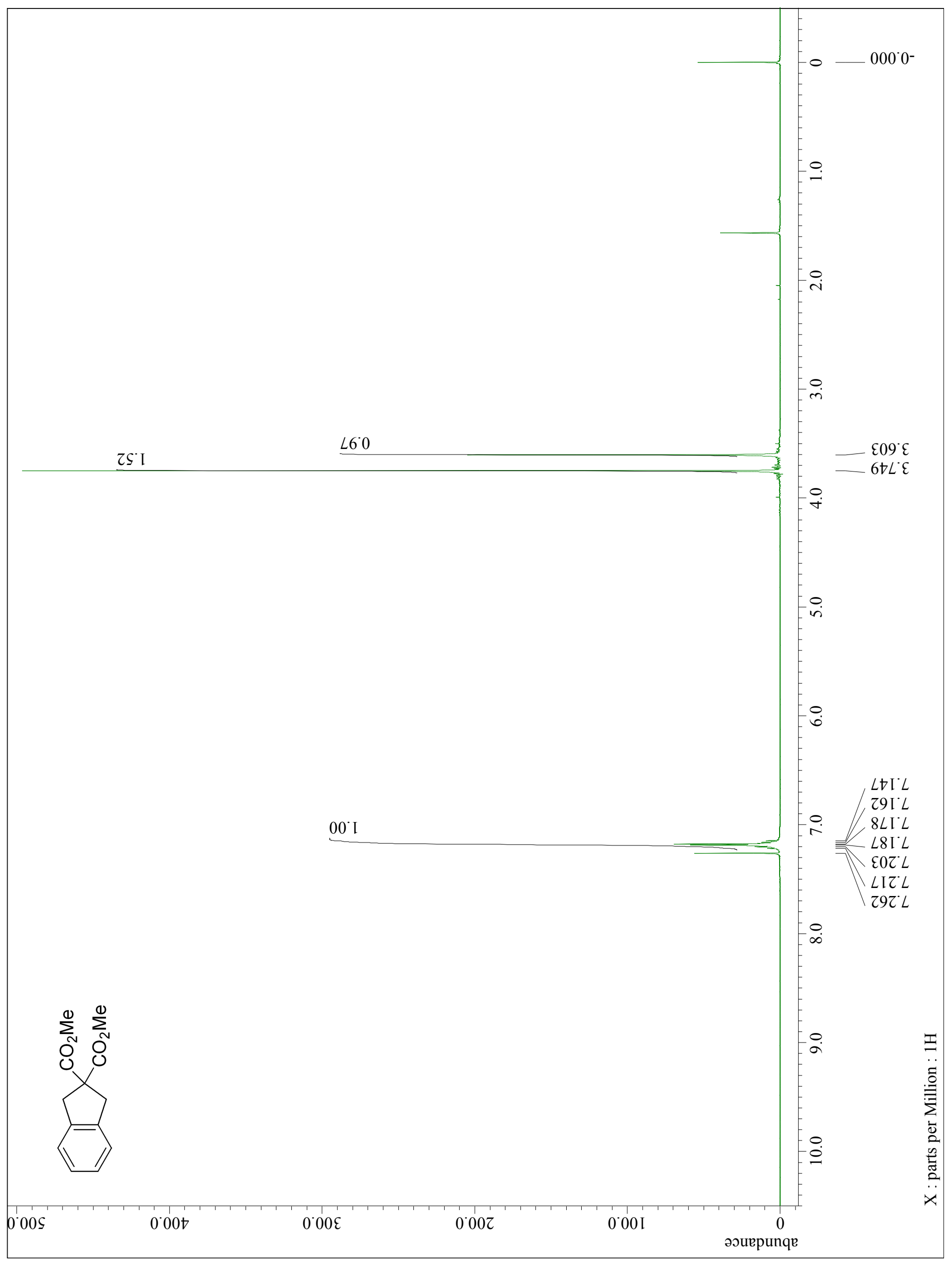




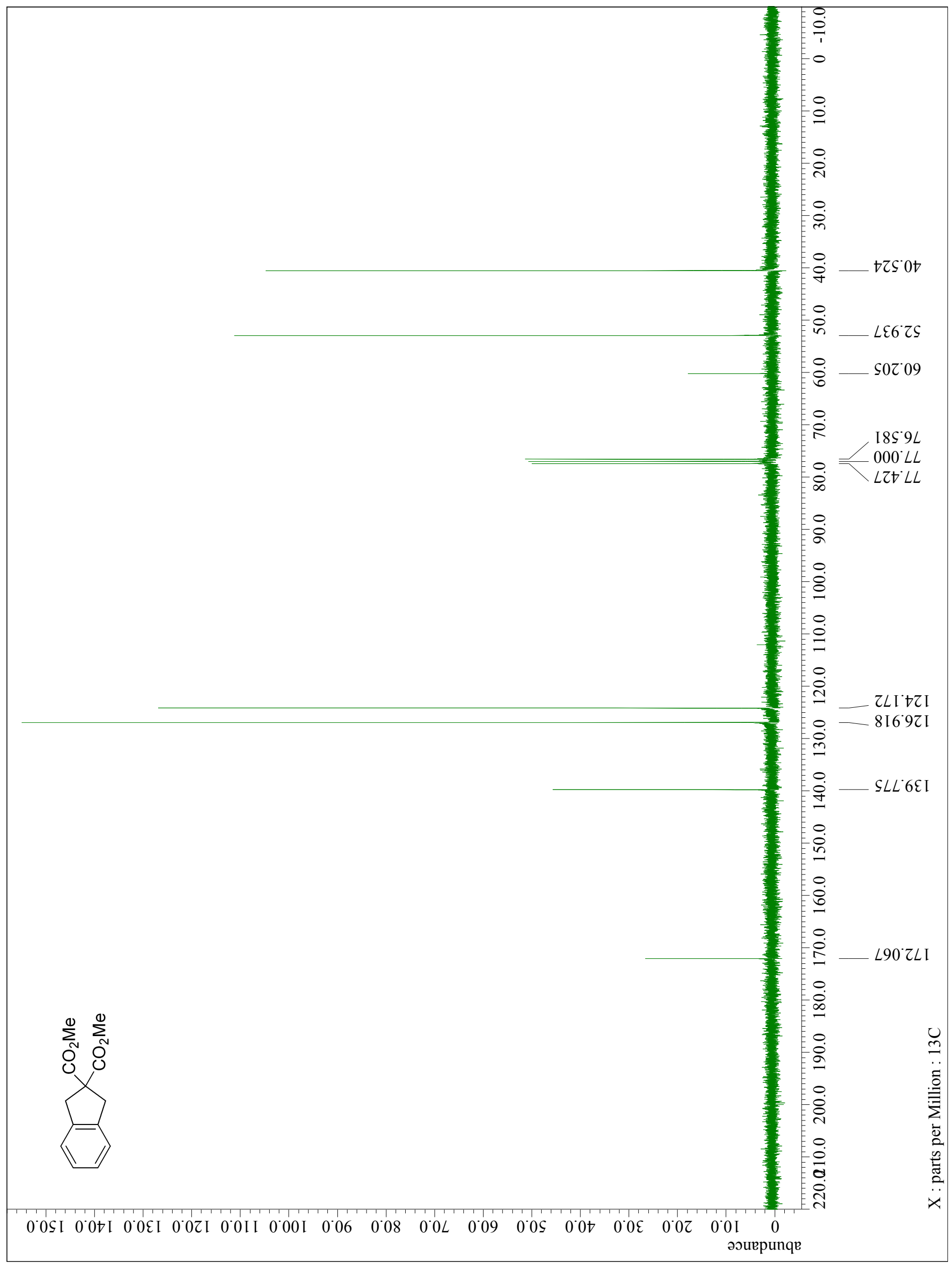


${ }^{1} \mathrm{H}$ NMR spectrum of d2-6a $\left(\mathrm{CDCl}_{3}, 300 \mathrm{MHz}\right)$.

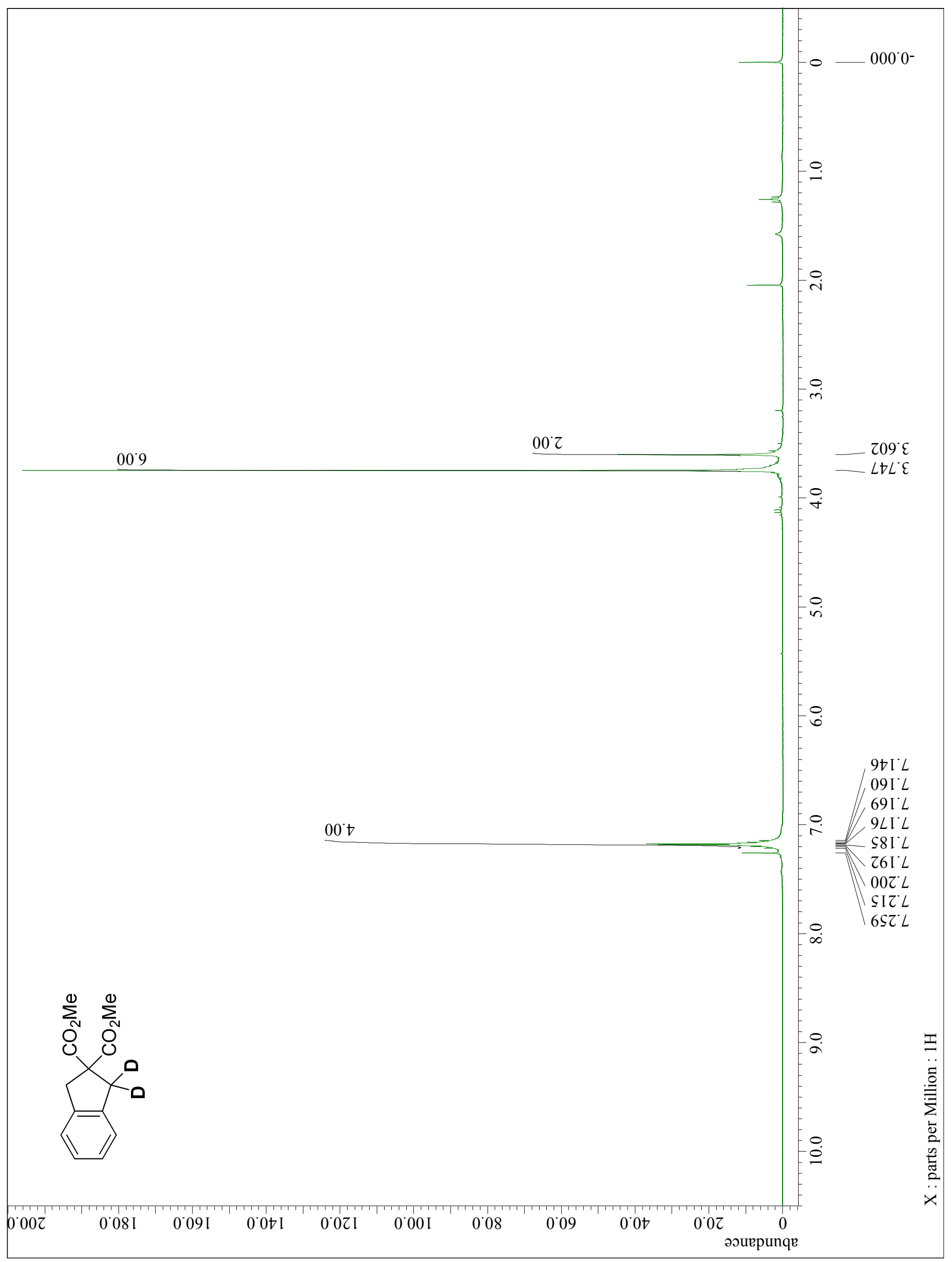


${ }^{1} \mathrm{H}$ NMR spectrum of $\mathbf{5 b}\left(\mathrm{CDCl}_{3}, 300 \mathrm{MHz}\right)$.

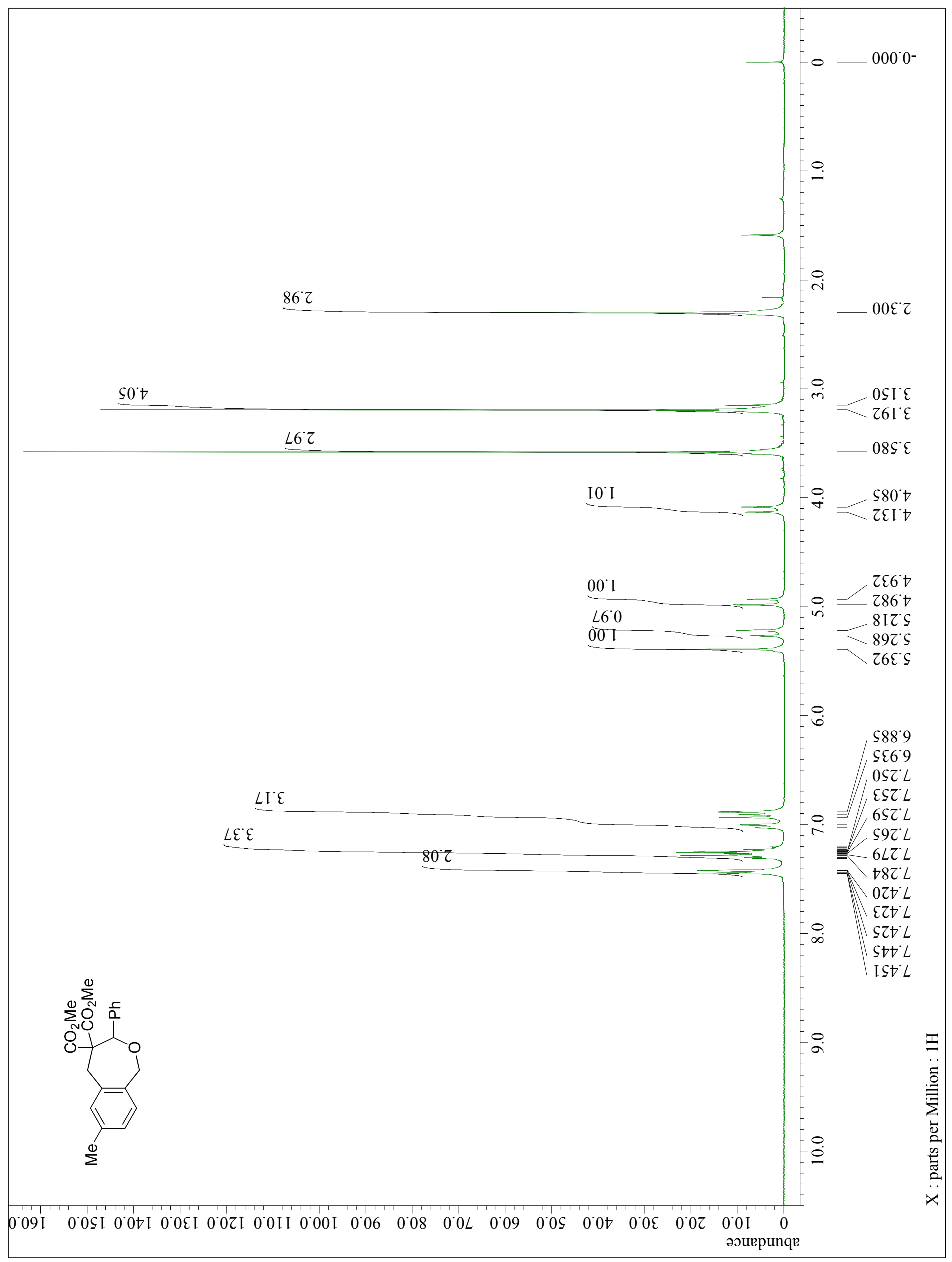


${ }^{13} \mathrm{C}$ NMR spectrum of $\mathbf{5 b}\left(\mathrm{CDCl}_{3}, 75 \mathrm{MHz}\right)$.

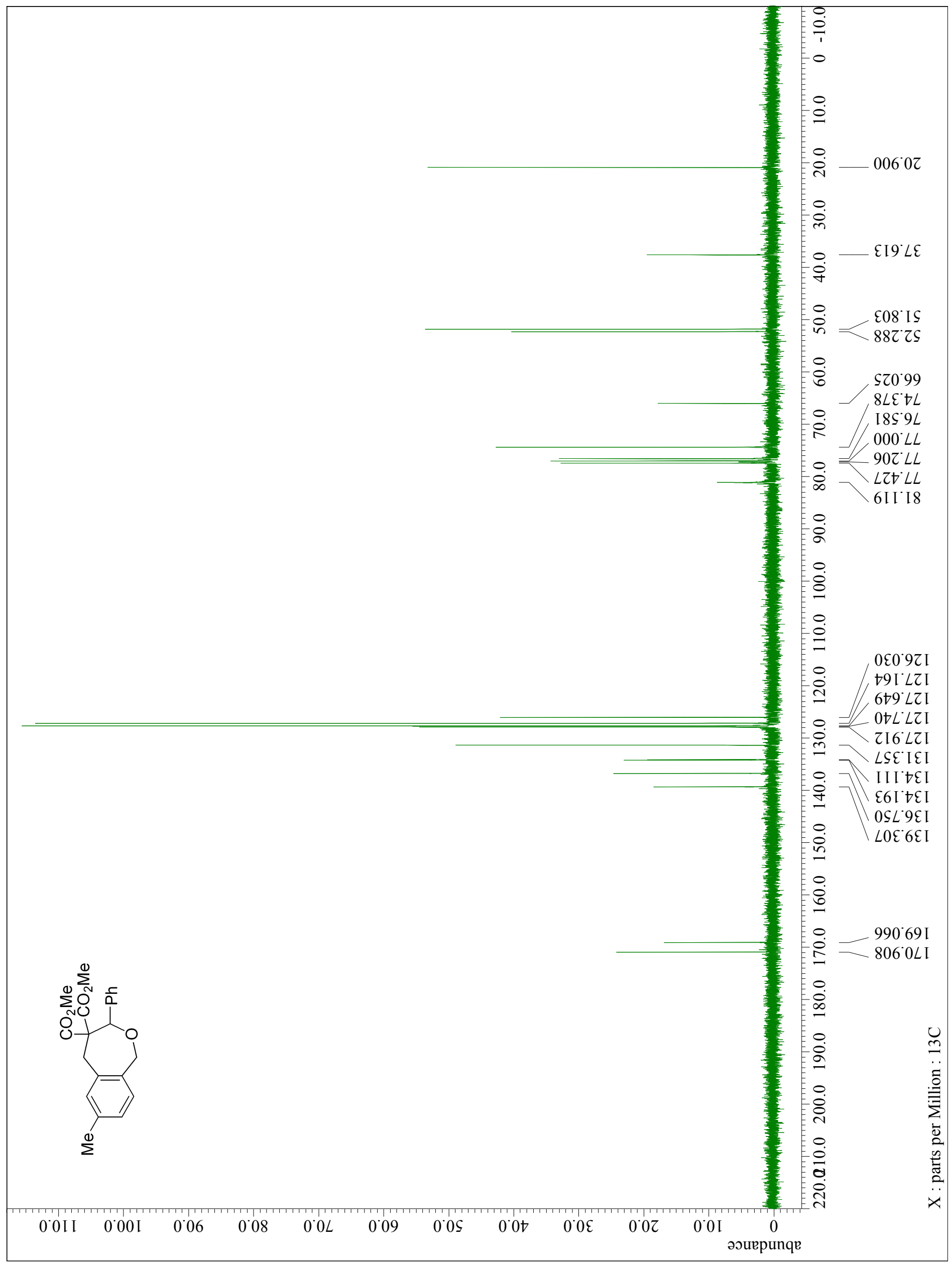


${ }^{1} \mathrm{H}$ NMR spectrum of $\mathbf{5 d}\left(\mathrm{CDCl}_{3}, 300 \mathrm{MHz}\right)$.

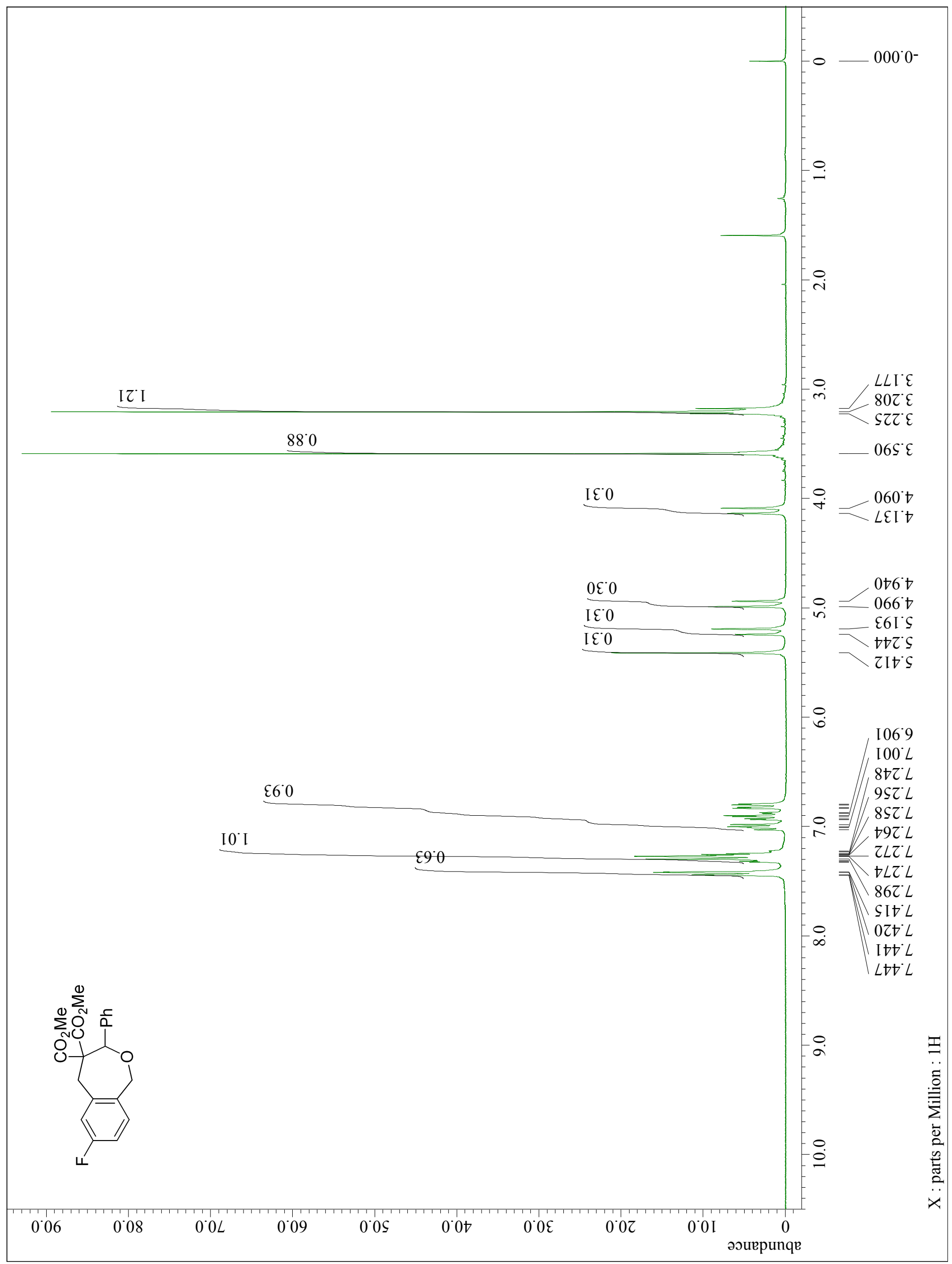




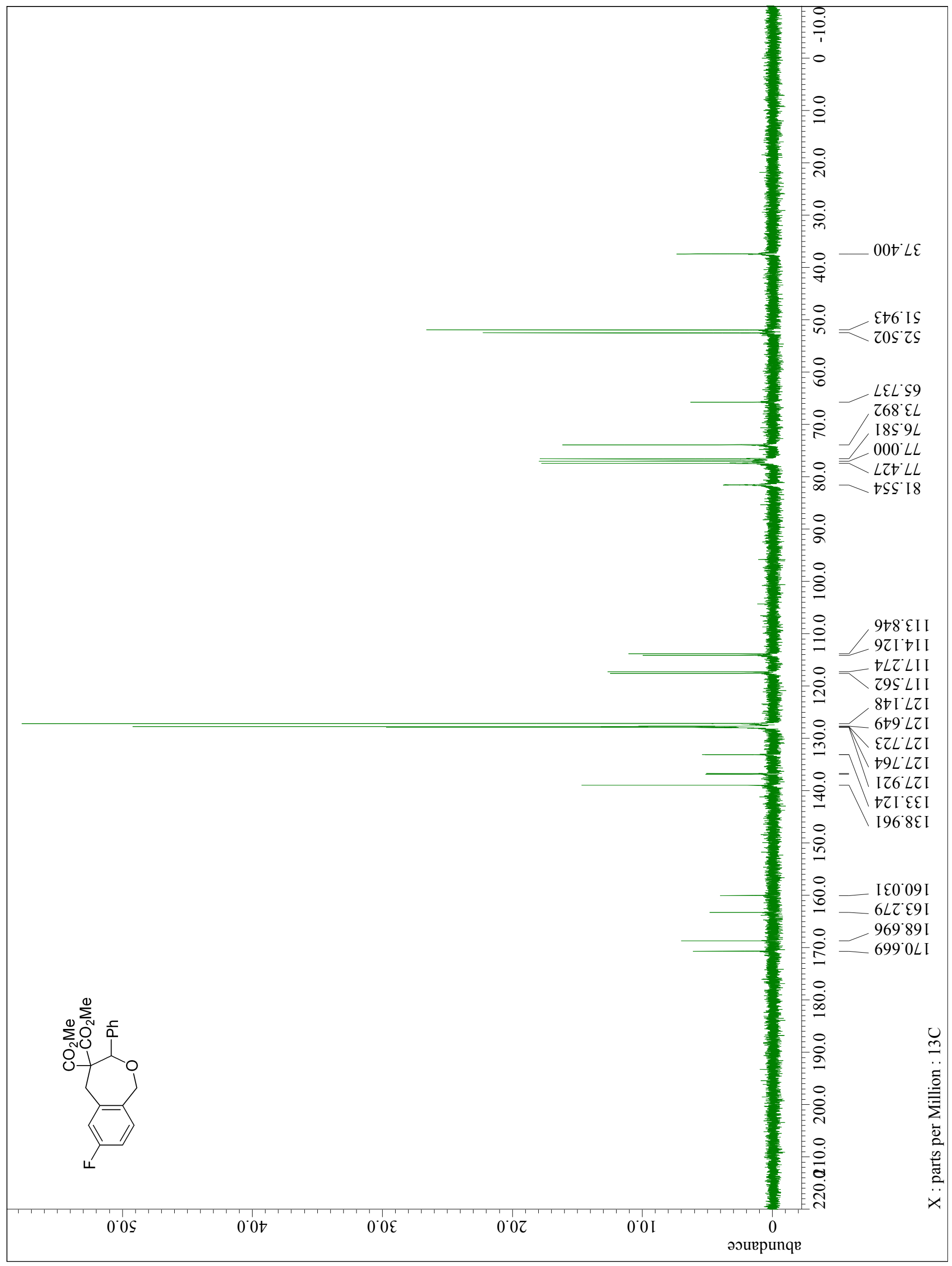


${ }^{19} \mathrm{~F}$ NMR spectrum of $\mathbf{5 d}\left(\mathrm{CDCl}_{3}, 283 \mathrm{MHz}\right)$.

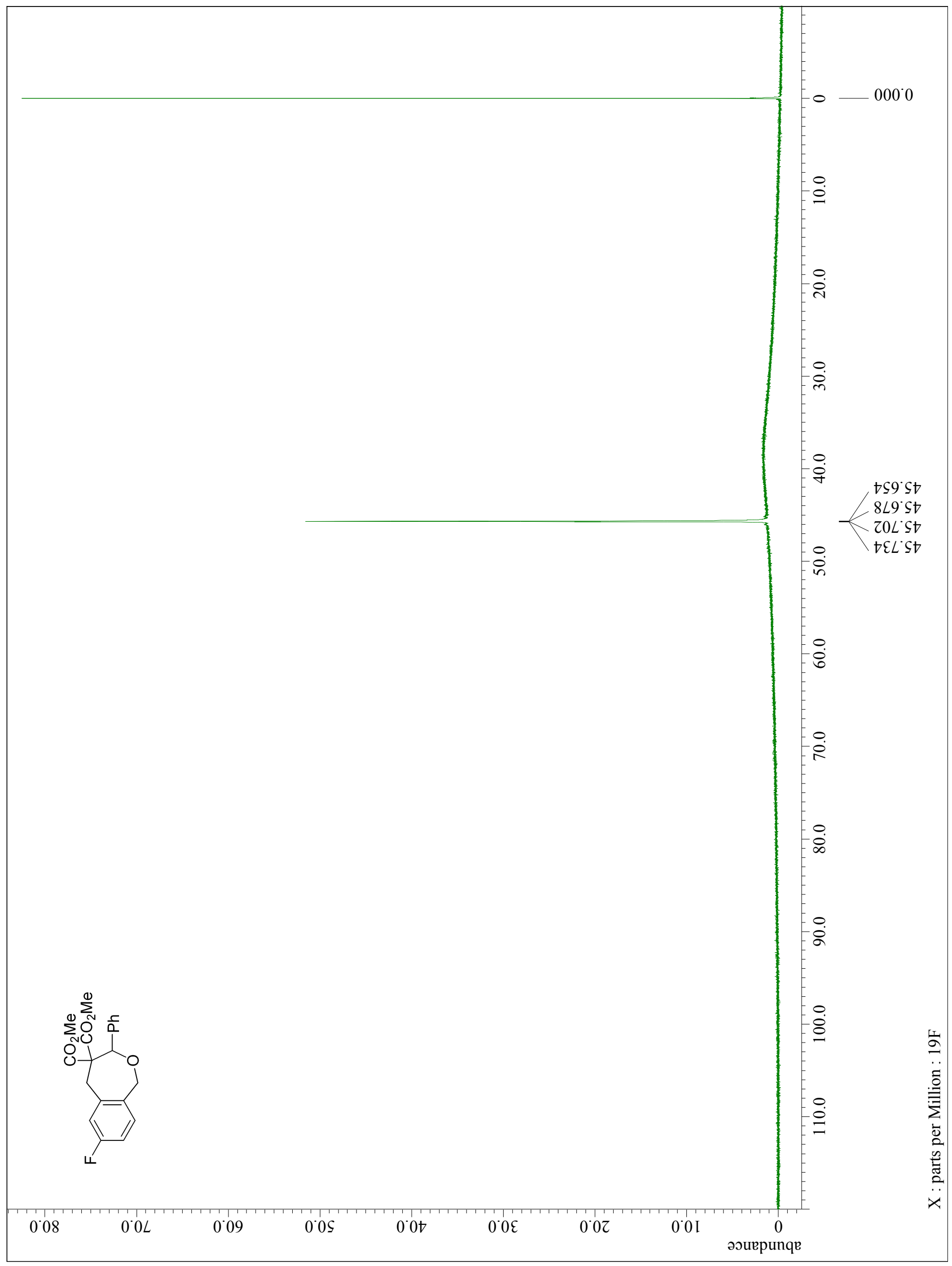


${ }^{1} \mathrm{H}$ NMR spectrum of $\mathbf{5 e}\left(\mathrm{CDCl}_{3}, 300 \mathrm{MHz}\right)$.

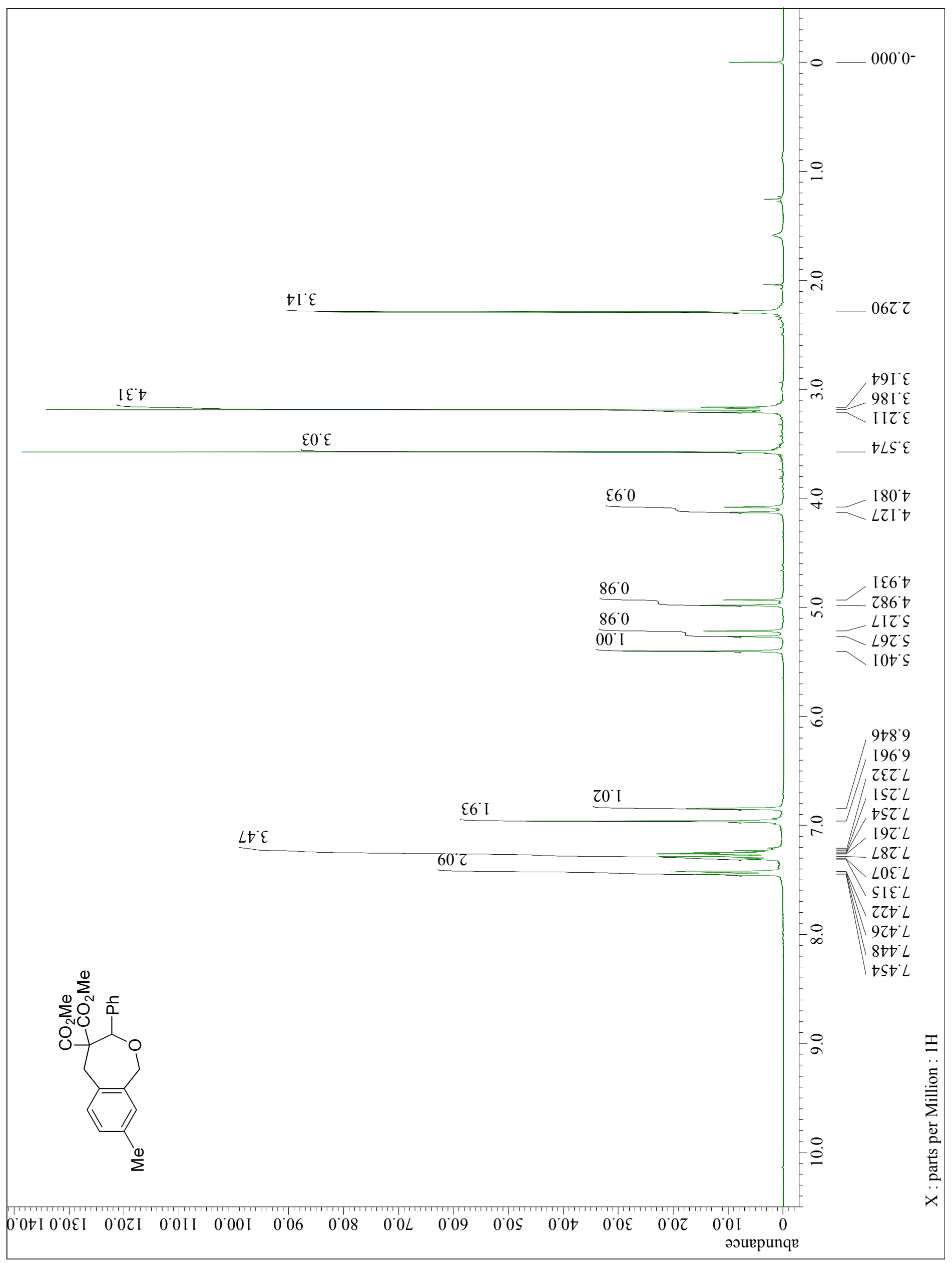


${ }^{13} \mathrm{C} \mathrm{NMR}$ spectrum of $\mathbf{5 e}\left(\mathrm{CDCl}_{3}, 75 \mathrm{MHz}\right)$.

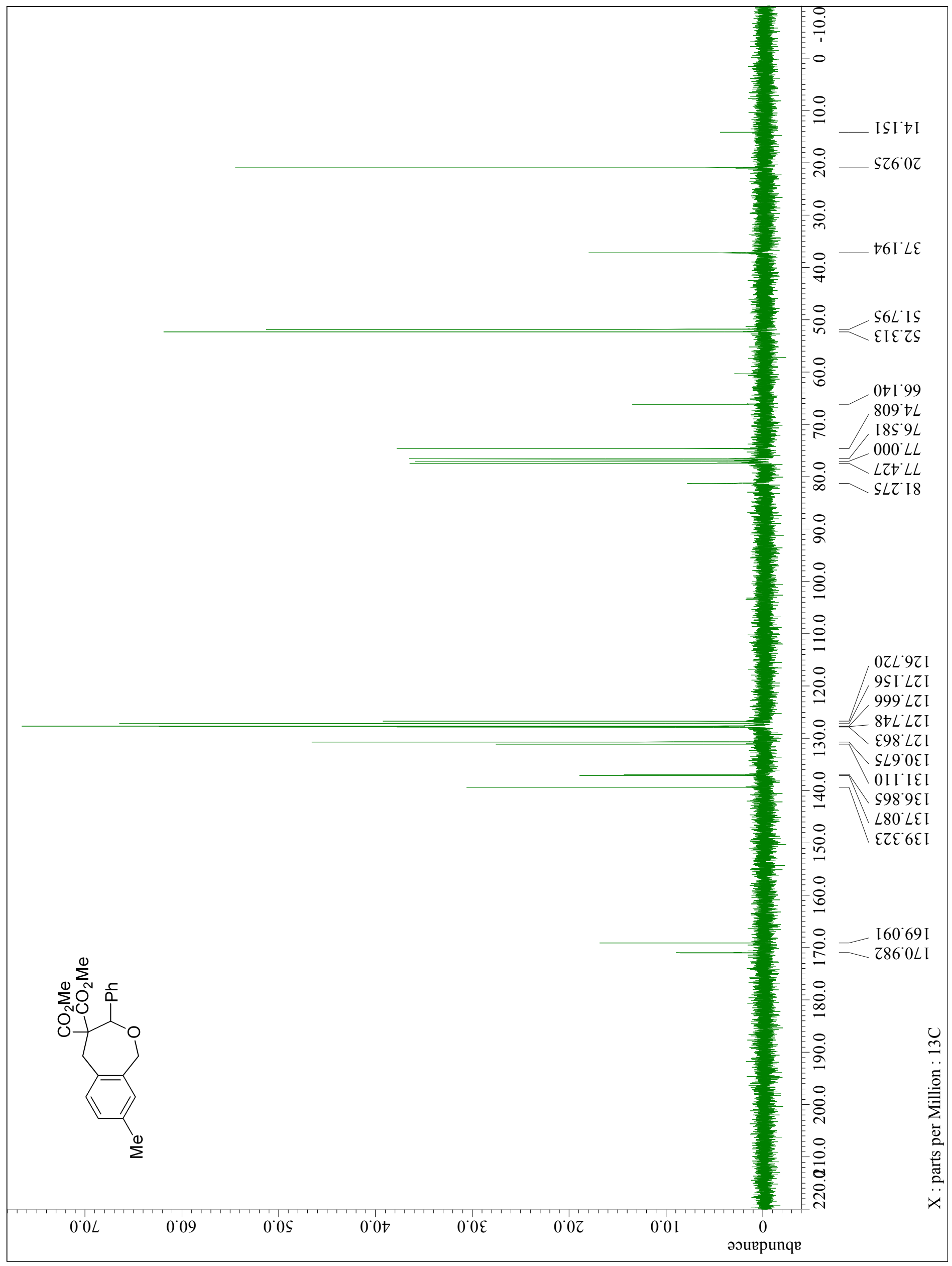


${ }^{1} \mathrm{H}$ NMR spectrum of $\mathbf{6 b}\left(\mathrm{CDCl}_{3}, 300 \mathrm{MHz}\right)$.

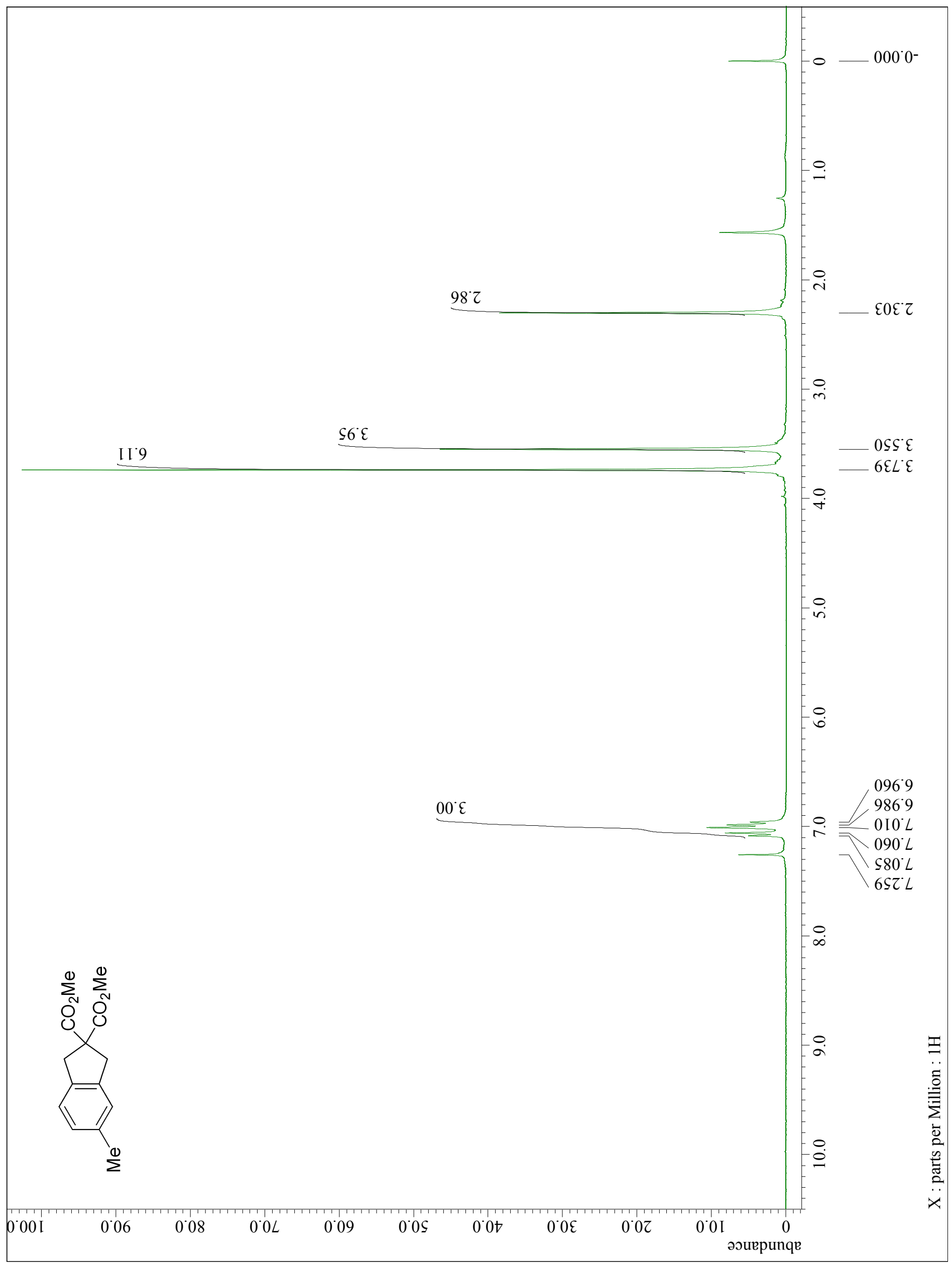


${ }^{13} \mathrm{C} \mathrm{NMR}$ spectrum of $\mathbf{6 b}\left(\mathrm{CDCl}_{3}, 75 \mathrm{MHz}\right)$.

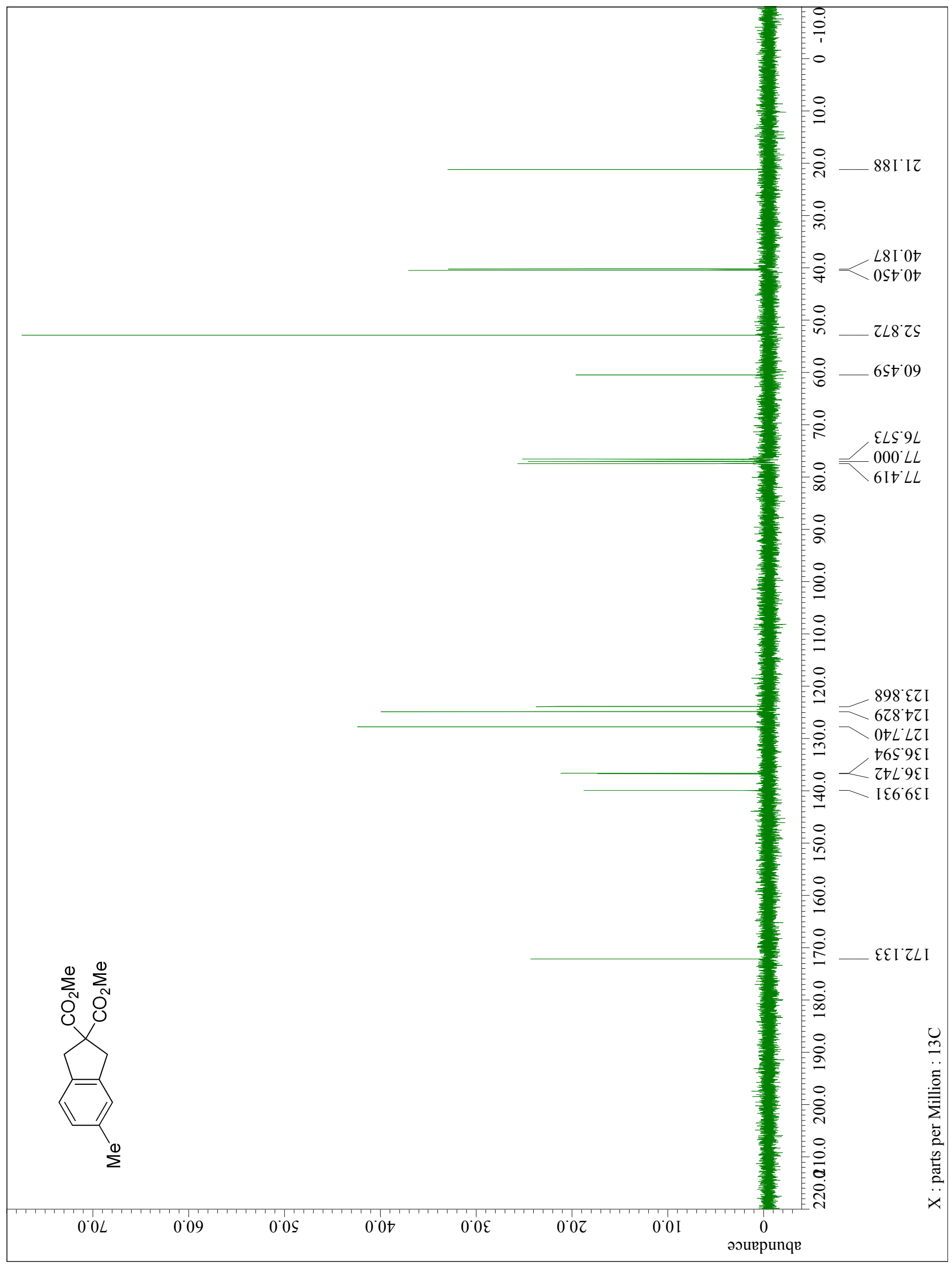




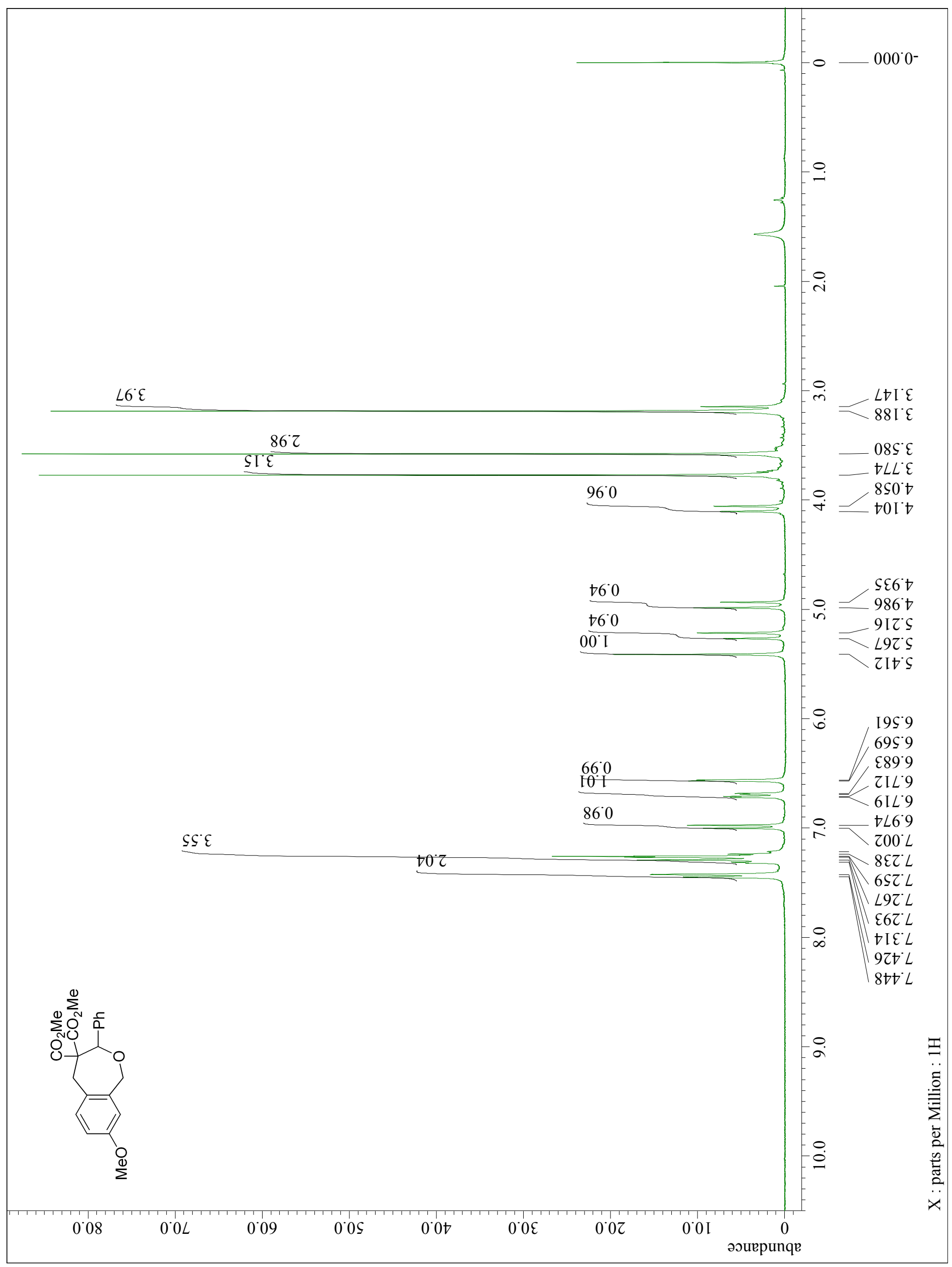


${ }^{13} \mathrm{C}$ NMR spectrum of $\mathbf{5 f}\left(\mathrm{CDCl}_{3}, 75 \mathrm{MHz}\right)$.

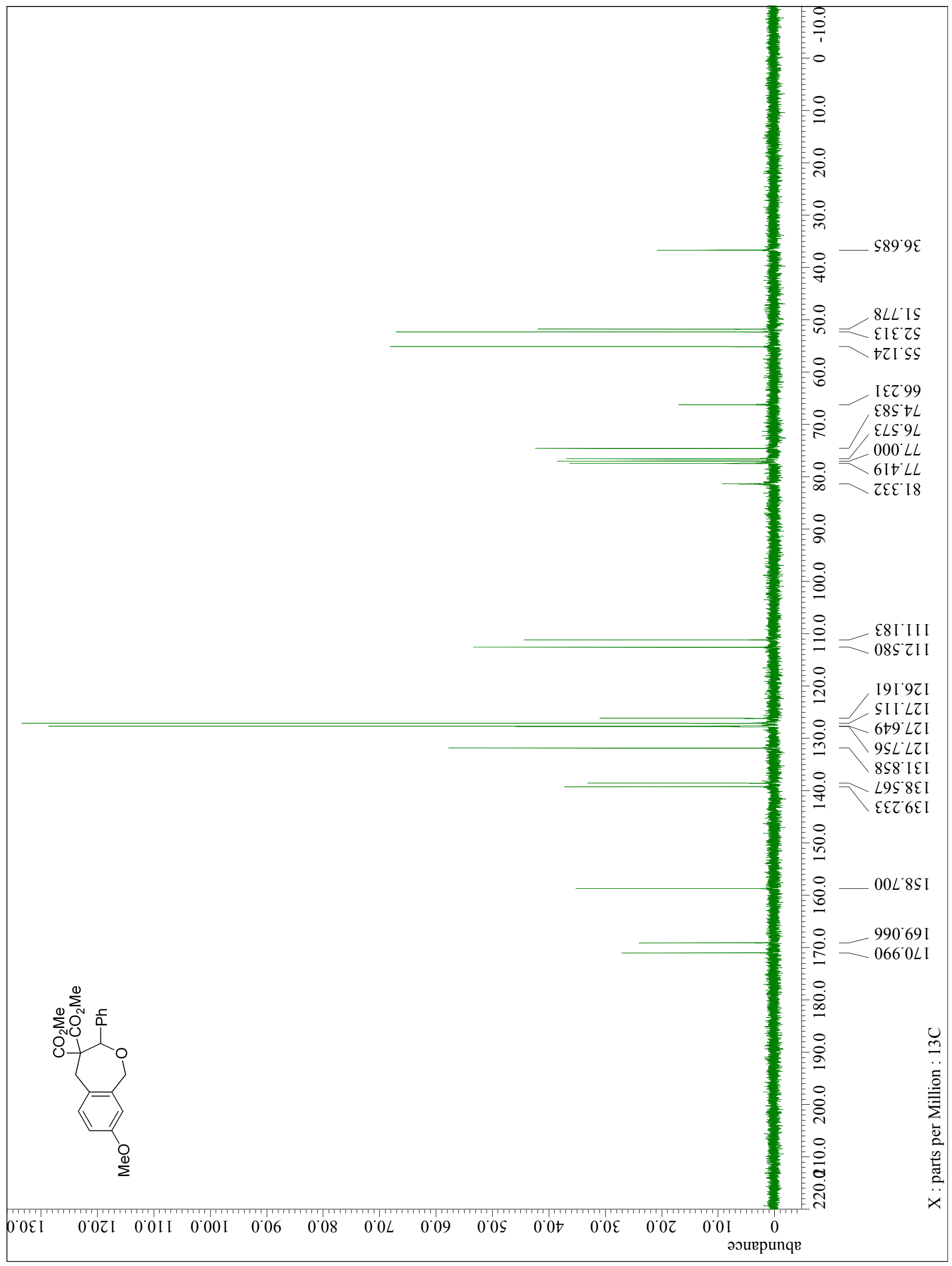


${ }^{1} \mathrm{H}$ NMR spectrum of $\mathbf{6 c}\left(\mathrm{CDCl}_{3}, 300 \mathrm{MHz}\right)$.

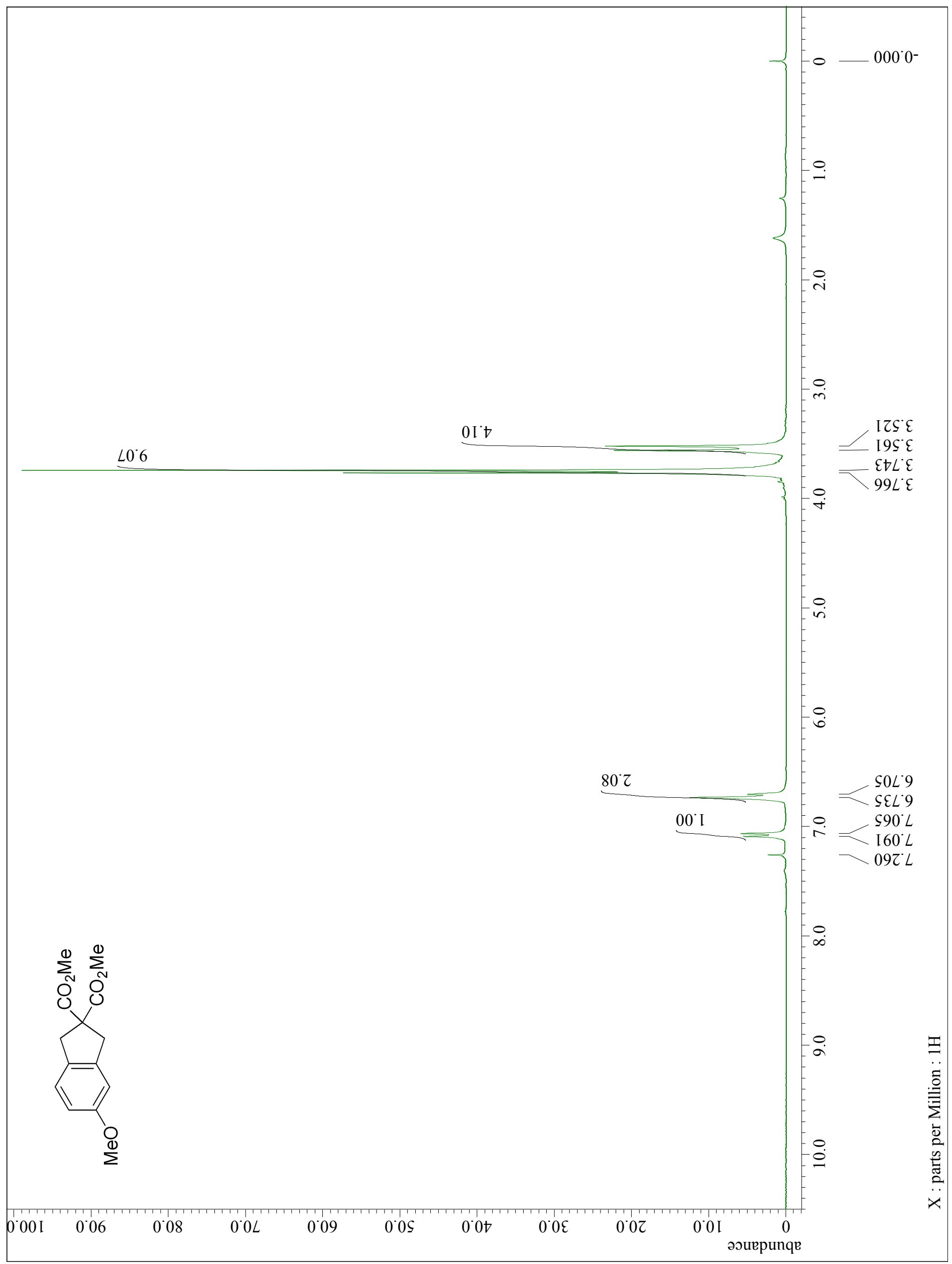


${ }^{13} \mathrm{C}$ NMR spectrum of $\mathbf{6 c}\left(\mathrm{CDCl}_{3}, 75 \mathrm{MHz}\right)$.

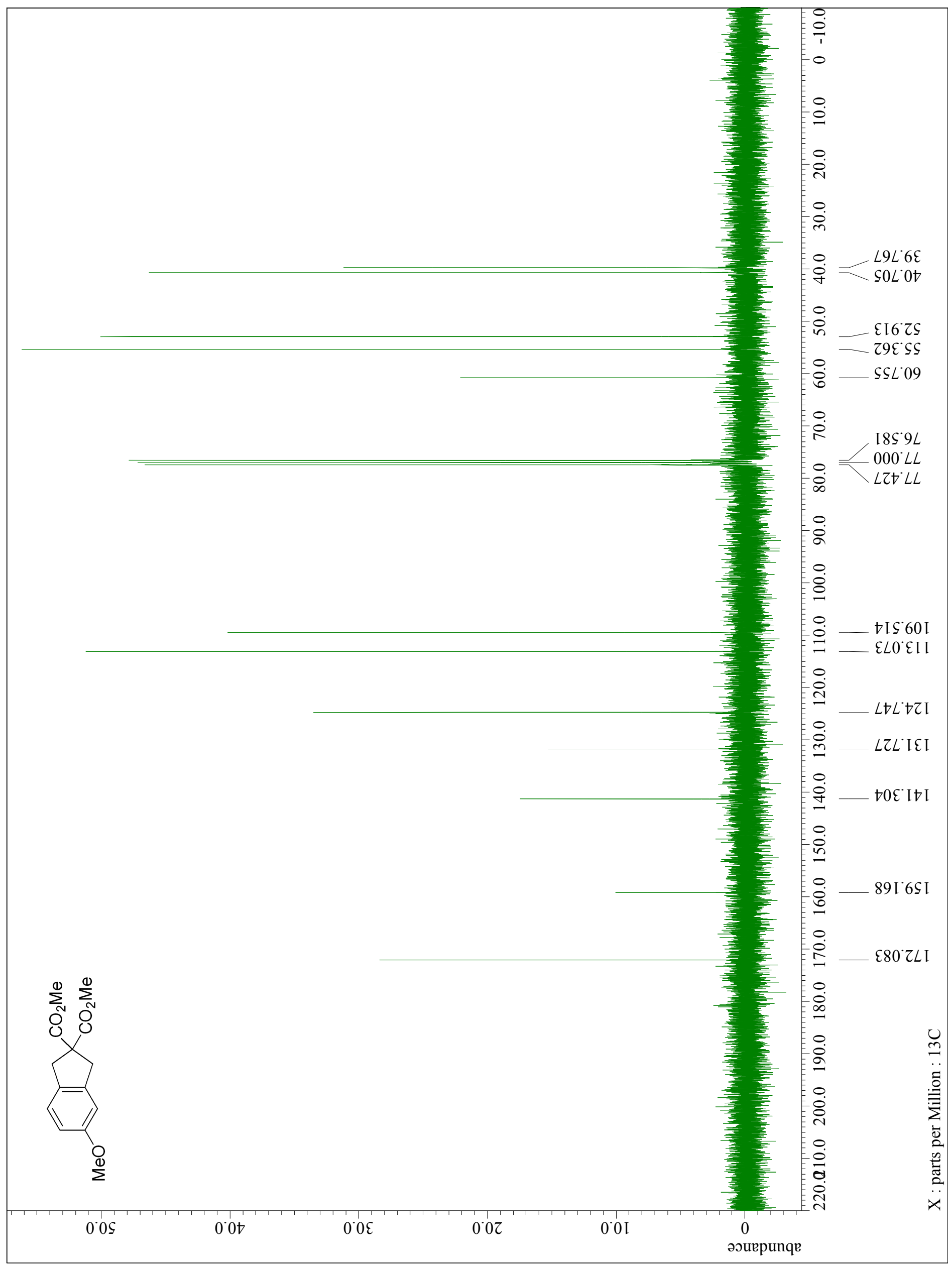


${ }^{1} \mathrm{H}$ NMR spectrum of $\mathbf{5 g}\left(\mathrm{CDCl}_{3}, 300 \mathrm{MHz}\right)$.

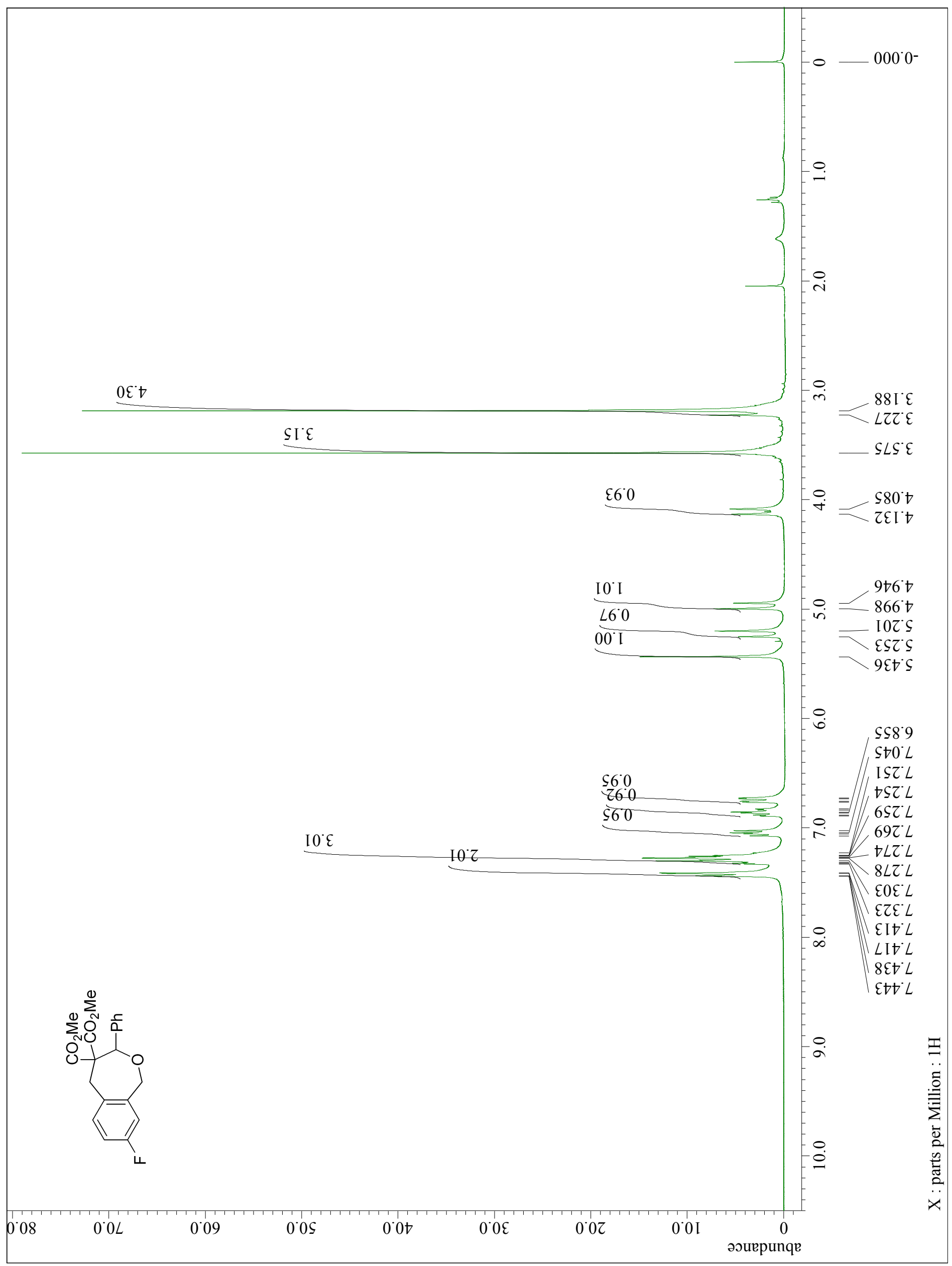




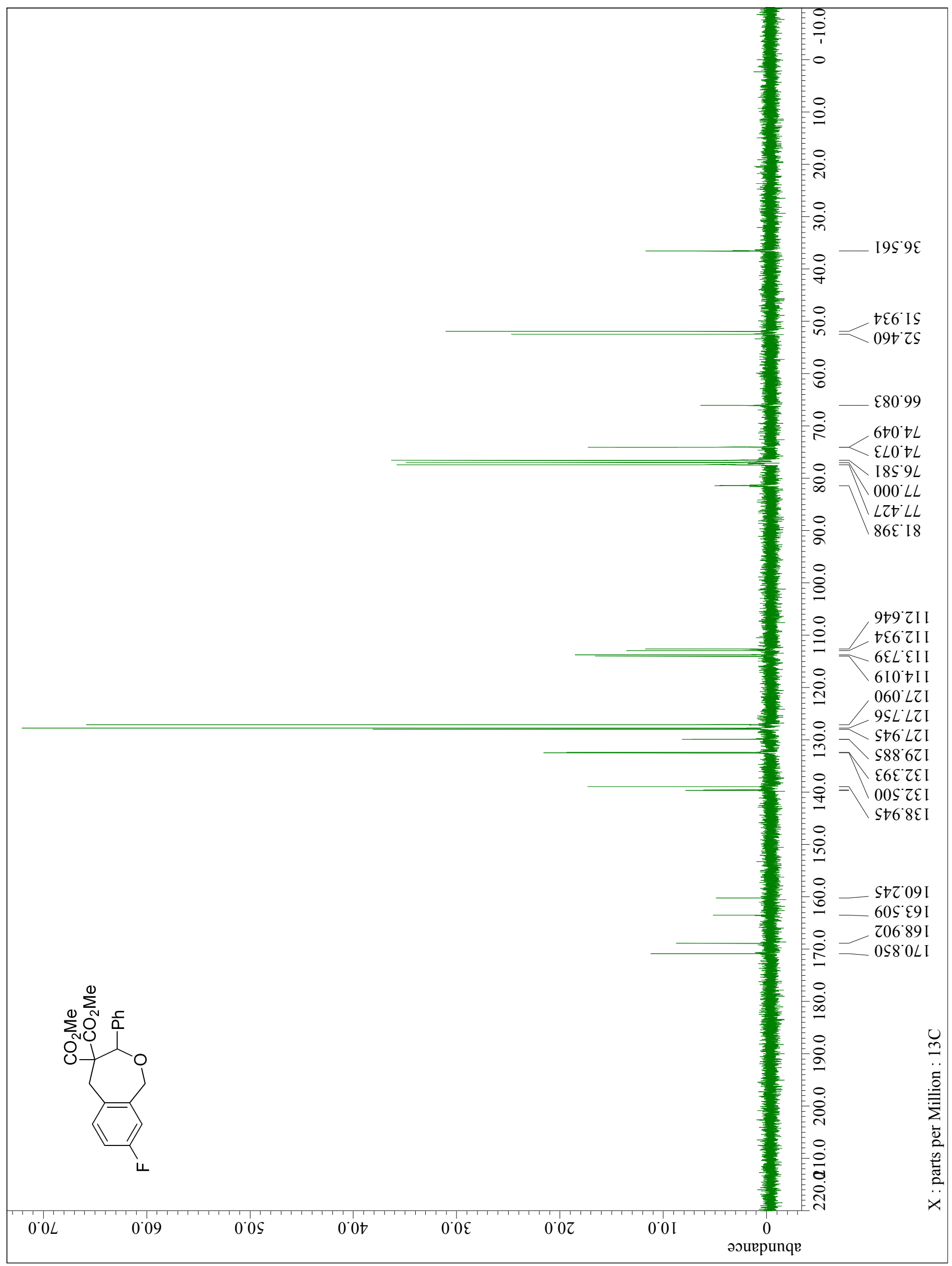


${ }^{19} \mathrm{~F}$ NMR spectrum of $\mathbf{5 g}\left(\mathrm{CDCl}_{3}, 283 \mathrm{MHz}\right)$.

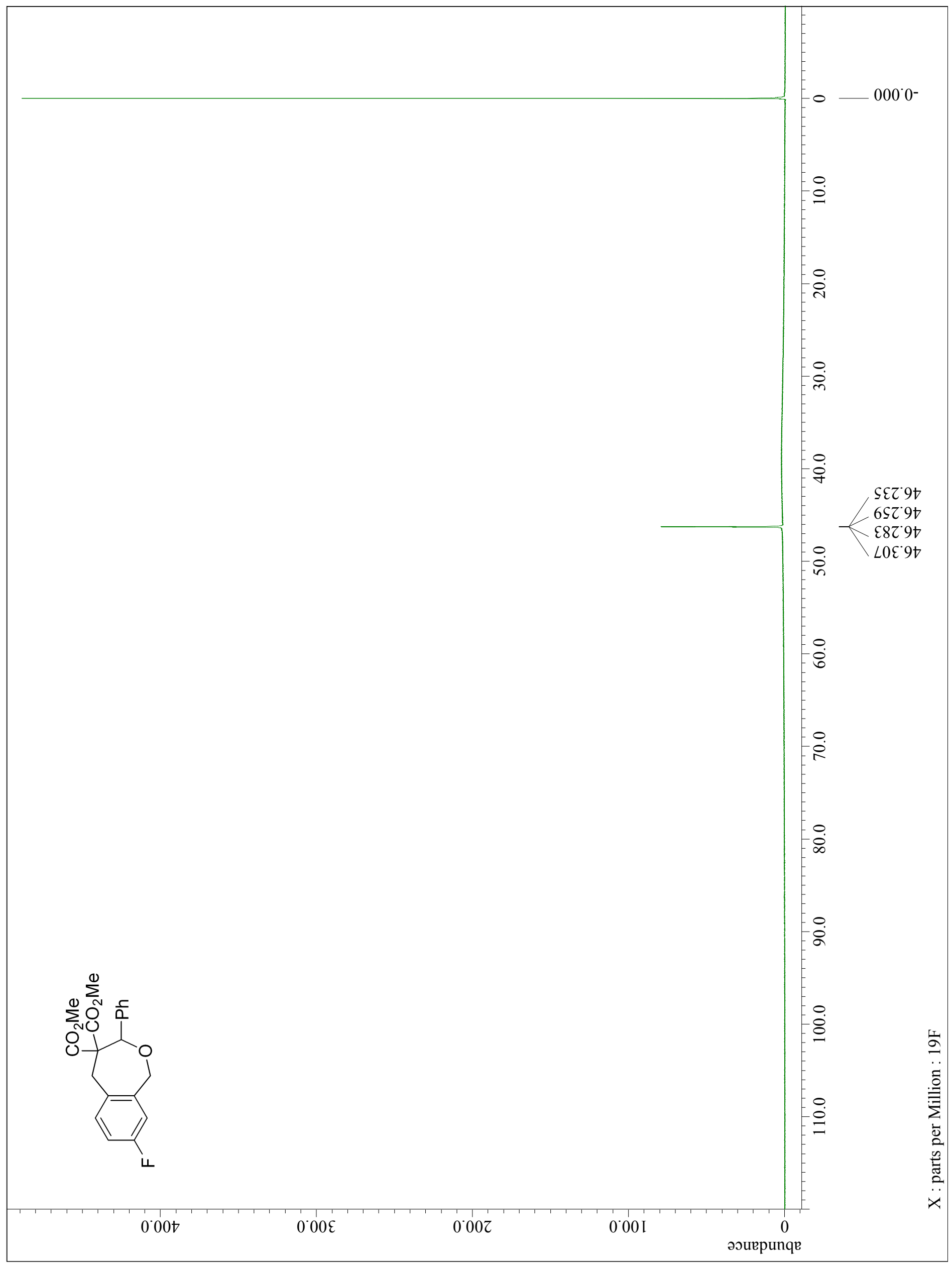


${ }^{1} \mathrm{H}$ NMR spectrum of $\mathbf{6 d}\left(\mathrm{CDCl}_{3}, 300 \mathrm{MHz}\right)$.

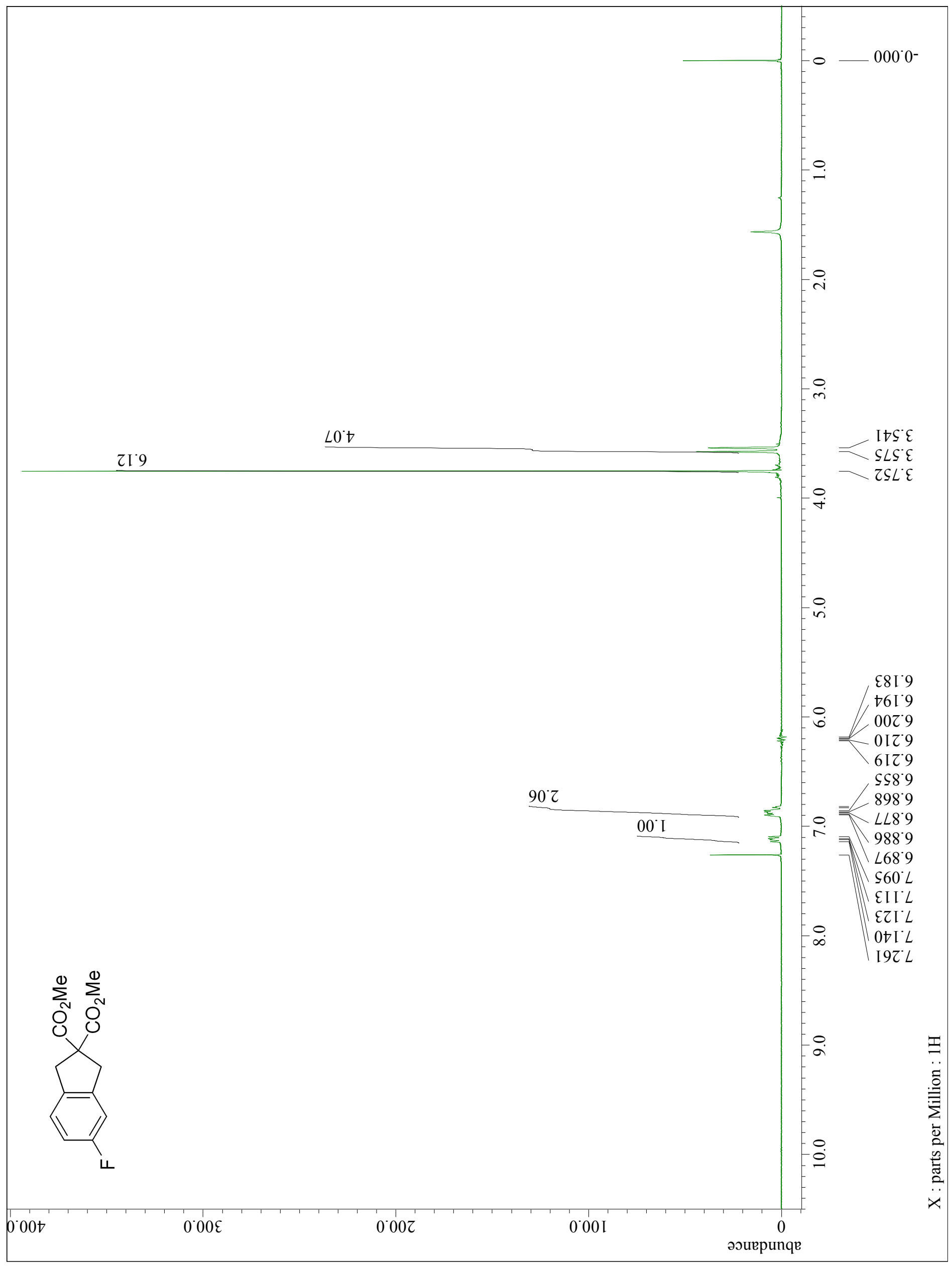


${ }^{13} \mathrm{C}$ NMR spectrum of $\mathbf{6 d}\left(\mathrm{CDCl}_{3}, 75 \mathrm{MHz}\right)$.

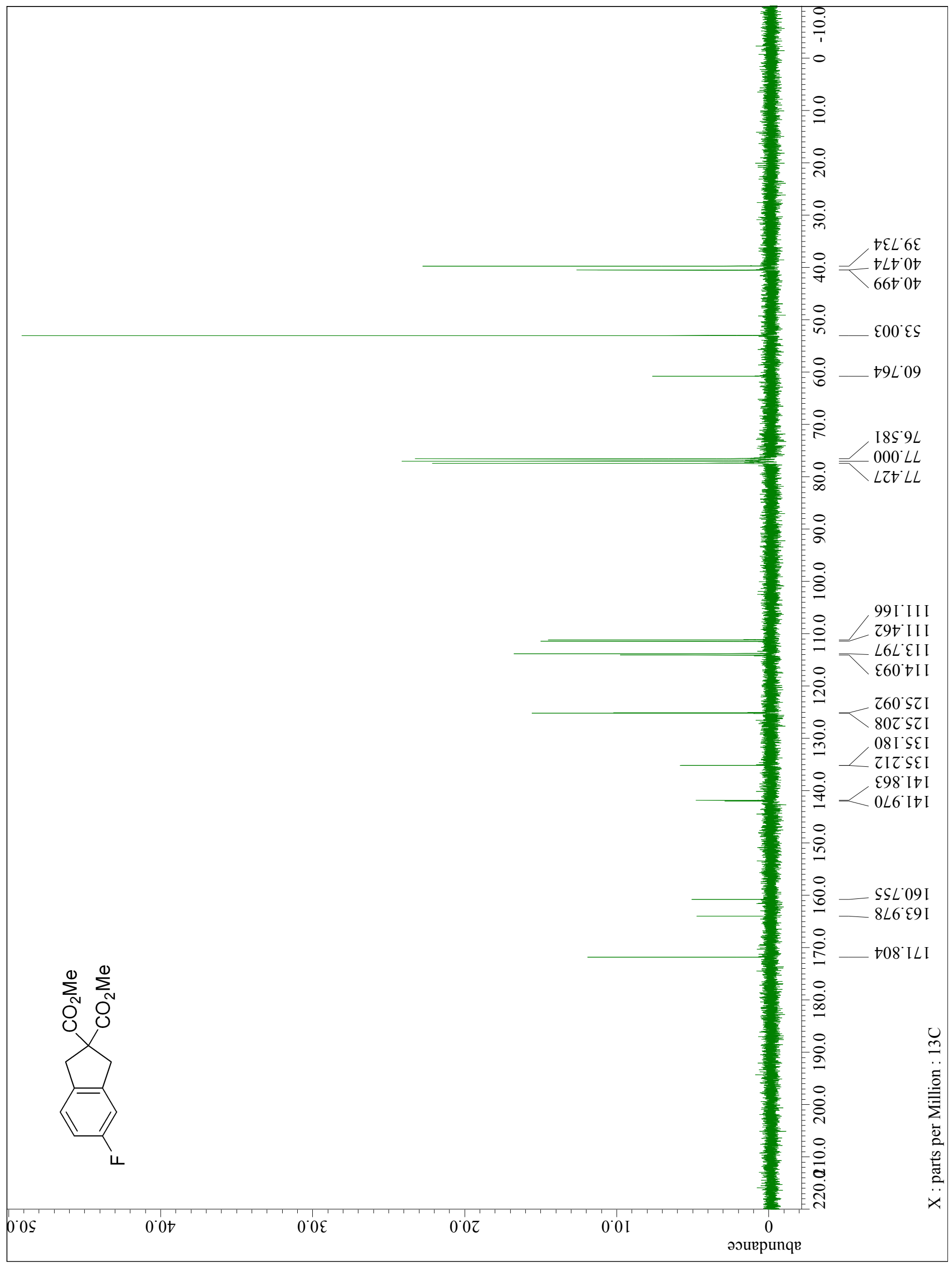


${ }^{19} \mathrm{~F}$ NMR spectrum of $\mathbf{6 d}\left(\mathrm{CDCl}_{3}, 283 \mathrm{MHz}\right)$.

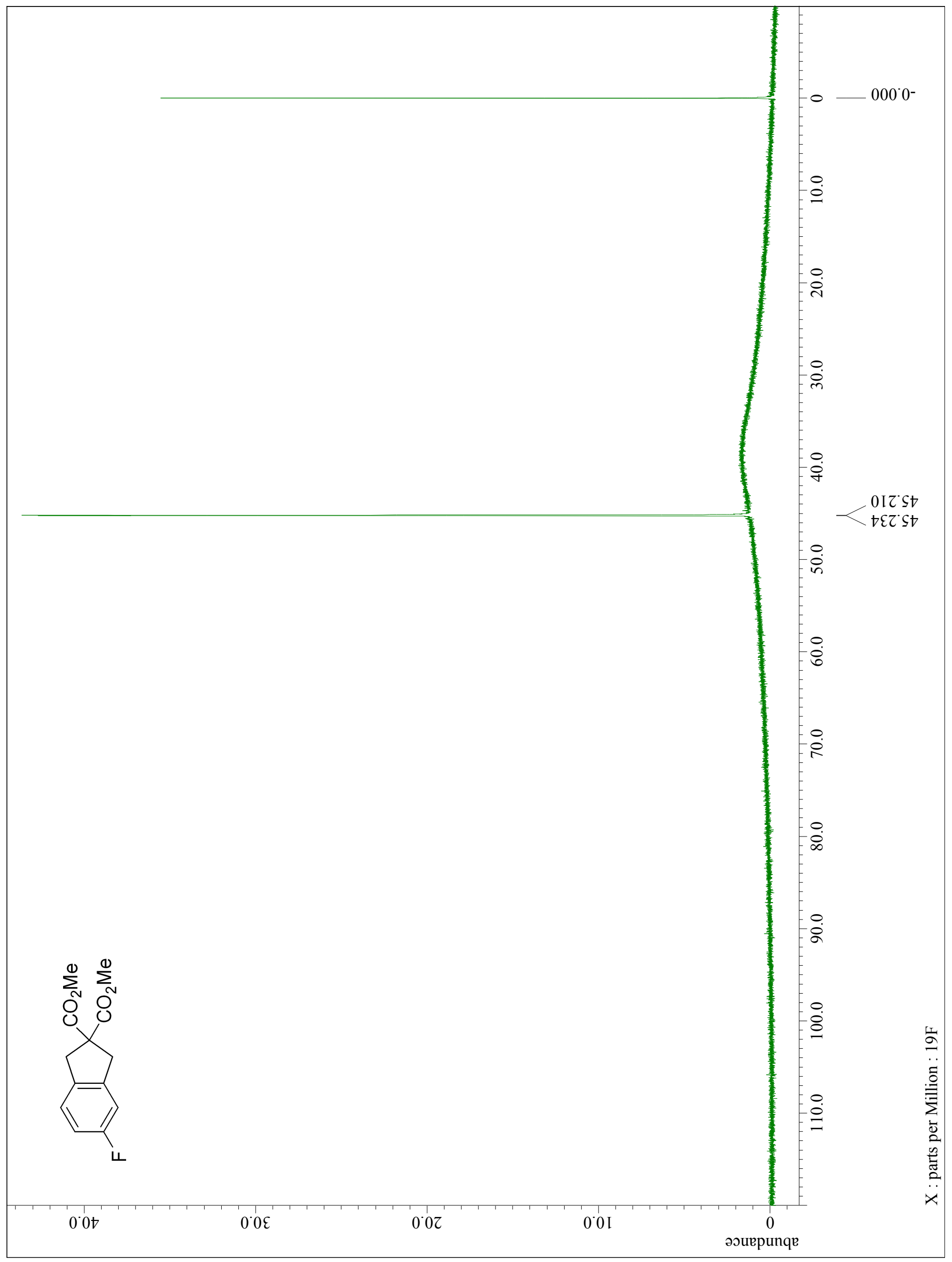


${ }^{1} \mathrm{H}$ NMR spectrum of $\mathbf{5 h}\left(\mathrm{CDCl}_{3}, 300 \mathrm{MHz}\right)$.

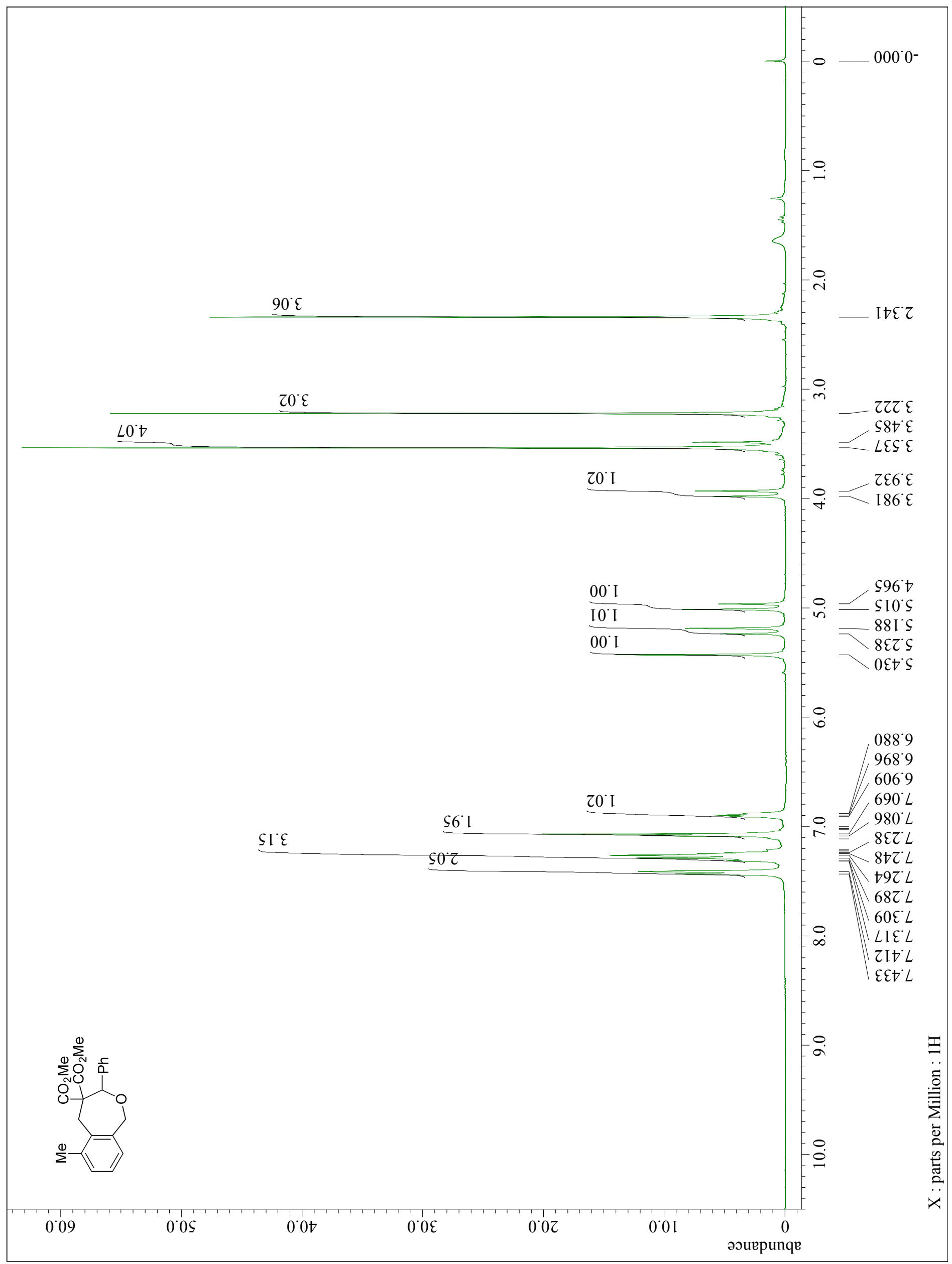


${ }^{13} \mathrm{C}$ NMR spectrum of $\mathbf{5 h}\left(\mathrm{CDCl}_{3}, 75 \mathrm{MHz}\right)$.

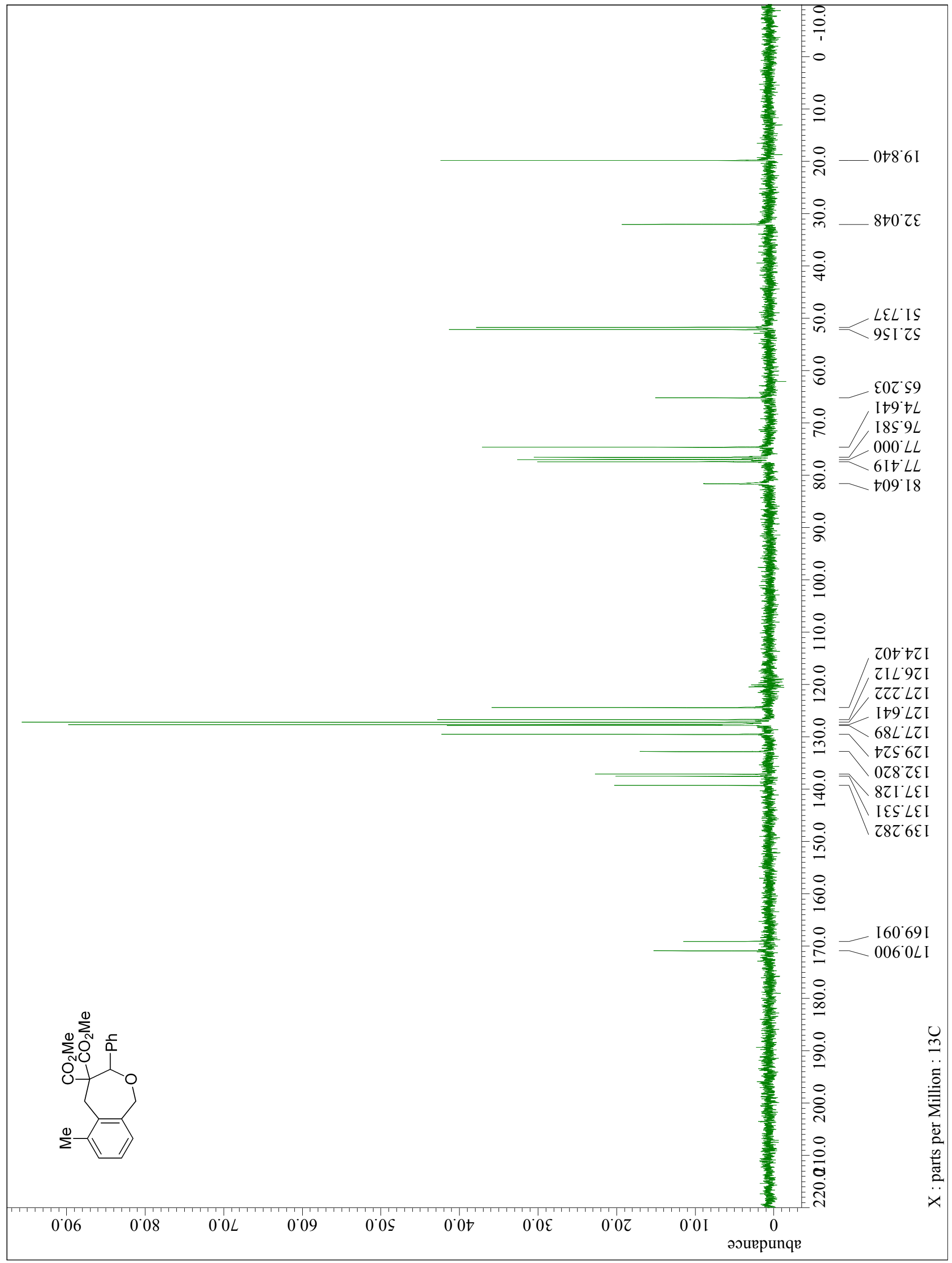


${ }^{1} \mathrm{H}$ NMR spectrum of $\mathbf{6 h}\left(\mathrm{CDCl}_{3}, 300 \mathrm{MHz}\right)$.

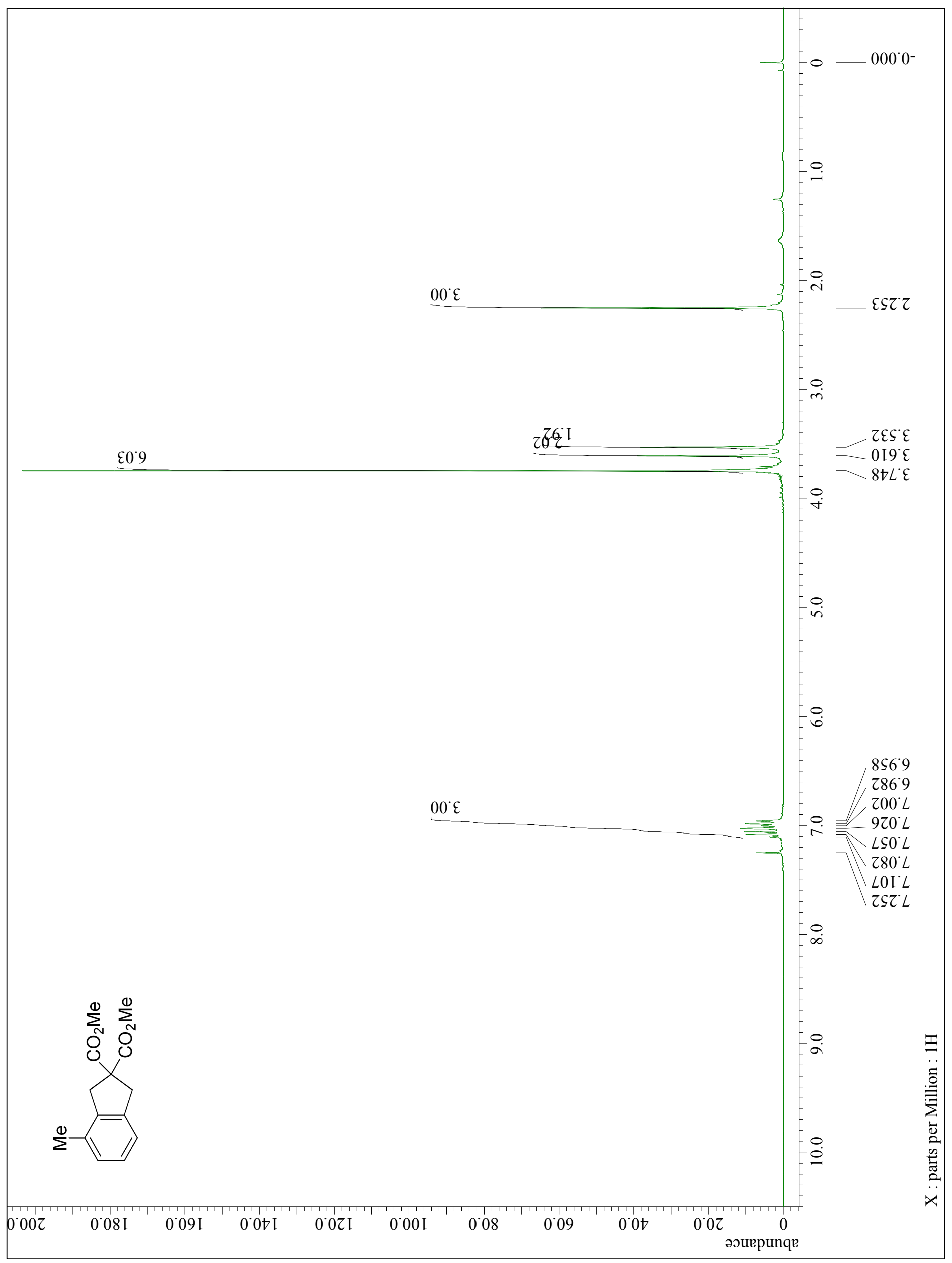




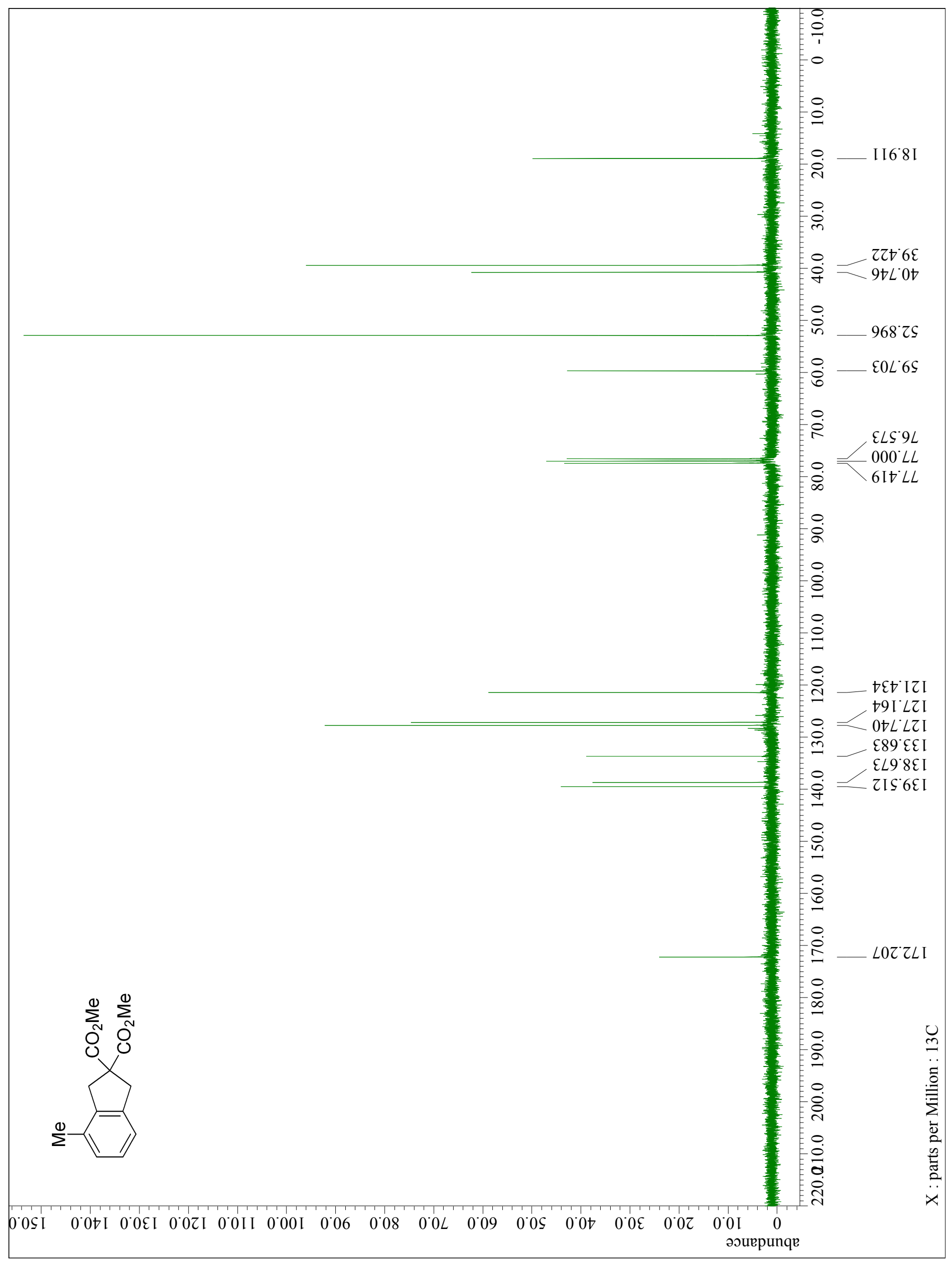


${ }^{1} \mathrm{H}$ NMR spectrum of $\mathbf{5 i}\left(\mathrm{CDCl}_{3}, 300 \mathrm{MHz}\right)$.

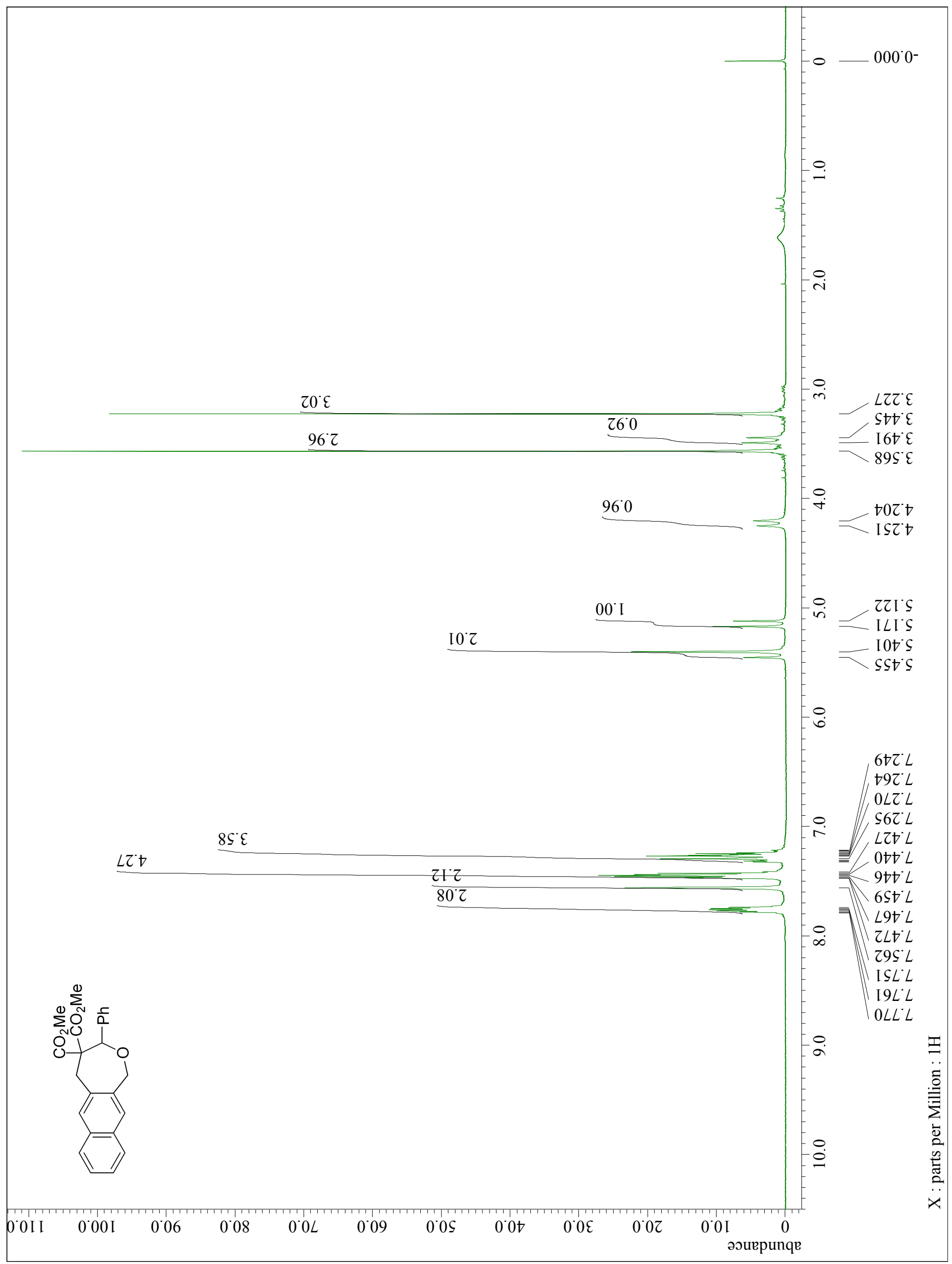


${ }^{13} \mathrm{C} \mathrm{NMR}$ spectrum of $\mathbf{5 i}\left(\mathrm{CDCl}_{3}, 100 \mathrm{MHz}\right)$.

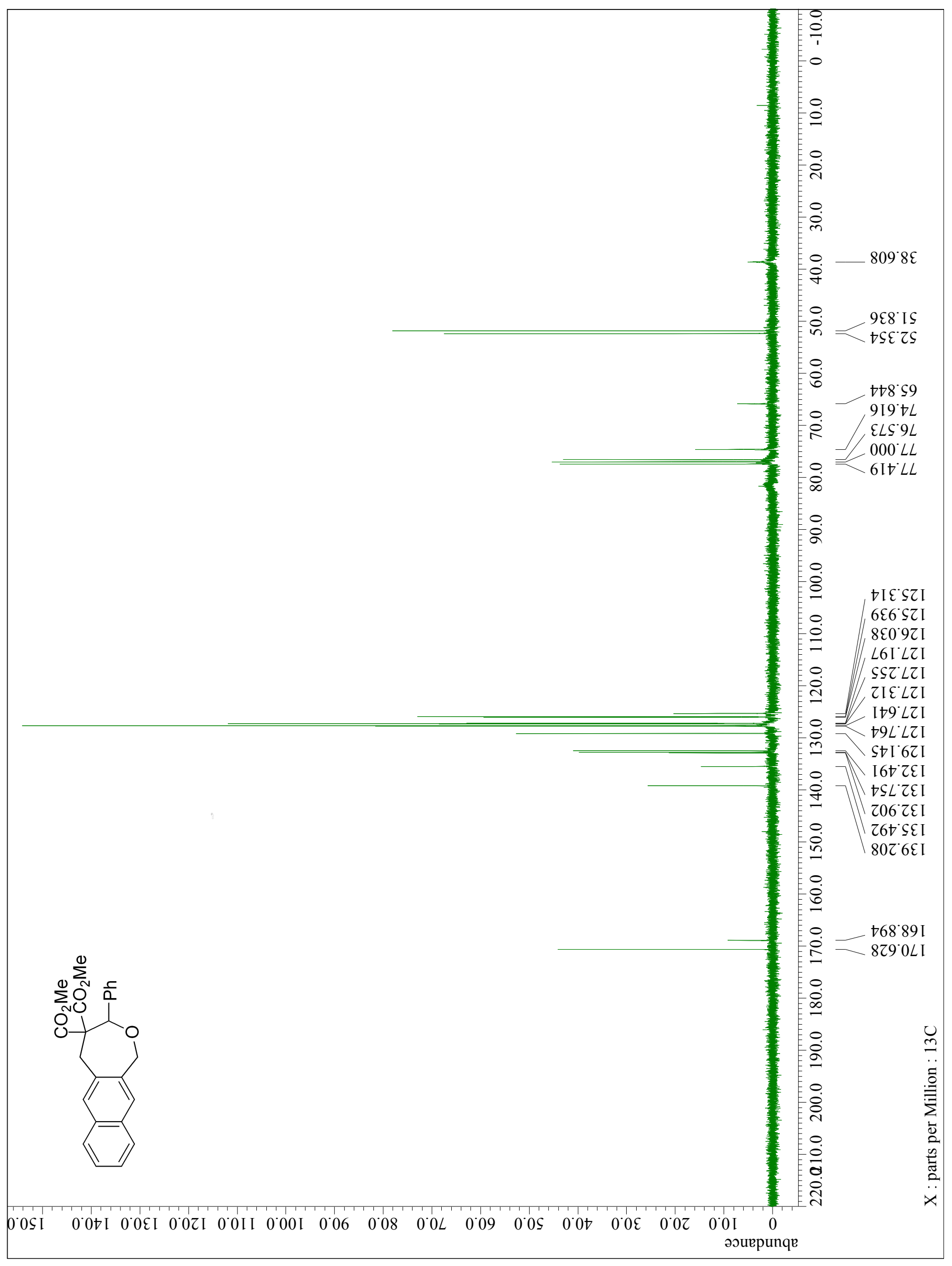


${ }^{1} \mathrm{H}$ NMR spectrum of $\mathbf{6 i}\left(\mathrm{CDCl}_{3}, 300 \mathrm{MHz}\right)$.

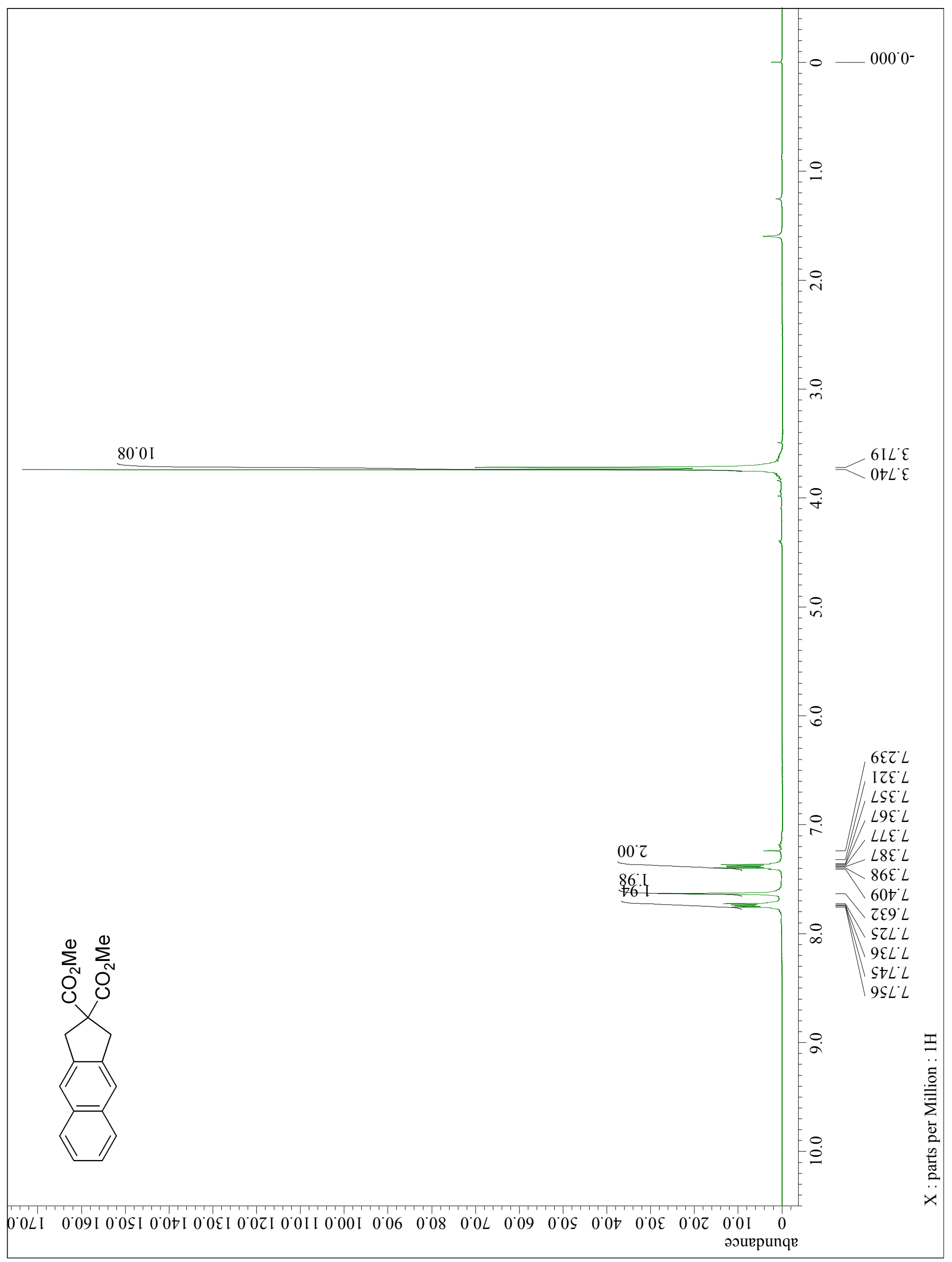


${ }^{13} \mathrm{C}$ NMR spectrum of $\mathbf{6} \mathbf{i}\left(\mathrm{CDCl}_{3}, 75 \mathrm{MHz}\right)$.

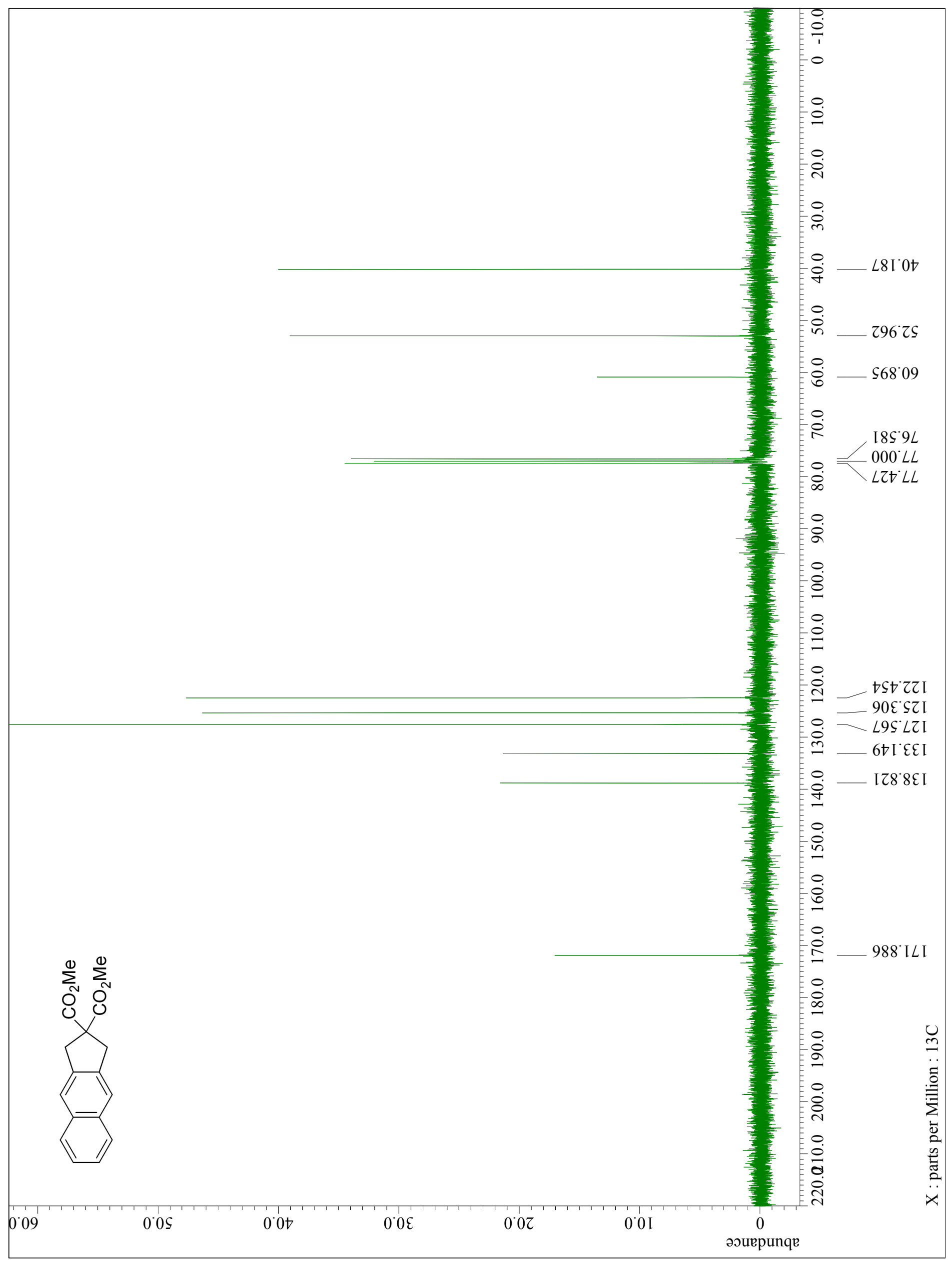




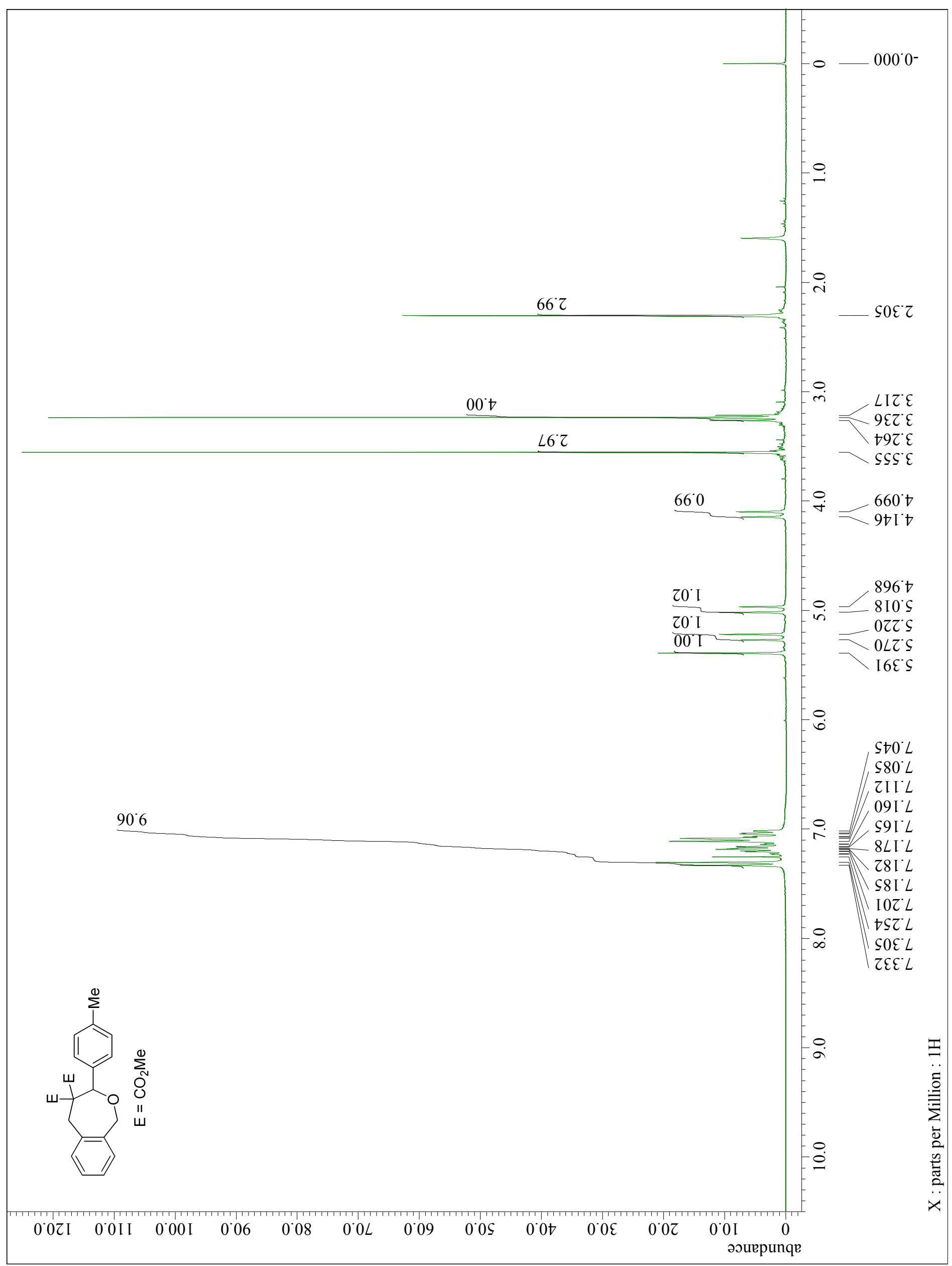




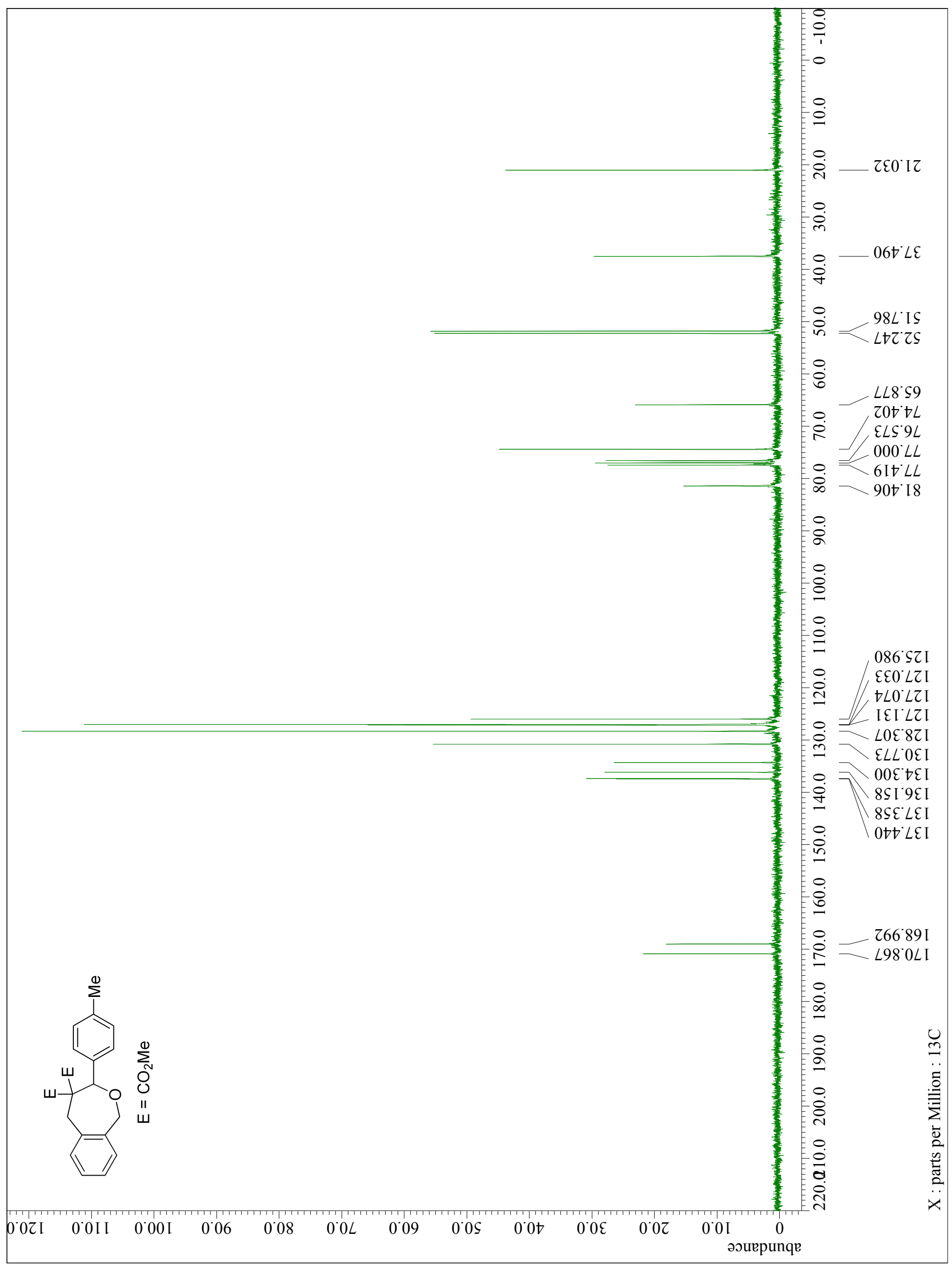


${ }^{1} \mathrm{H}$ NMR spectrum of $\mathbf{5 k}\left(\mathrm{CDCl}_{3}, 300 \mathrm{MHz}\right)$.

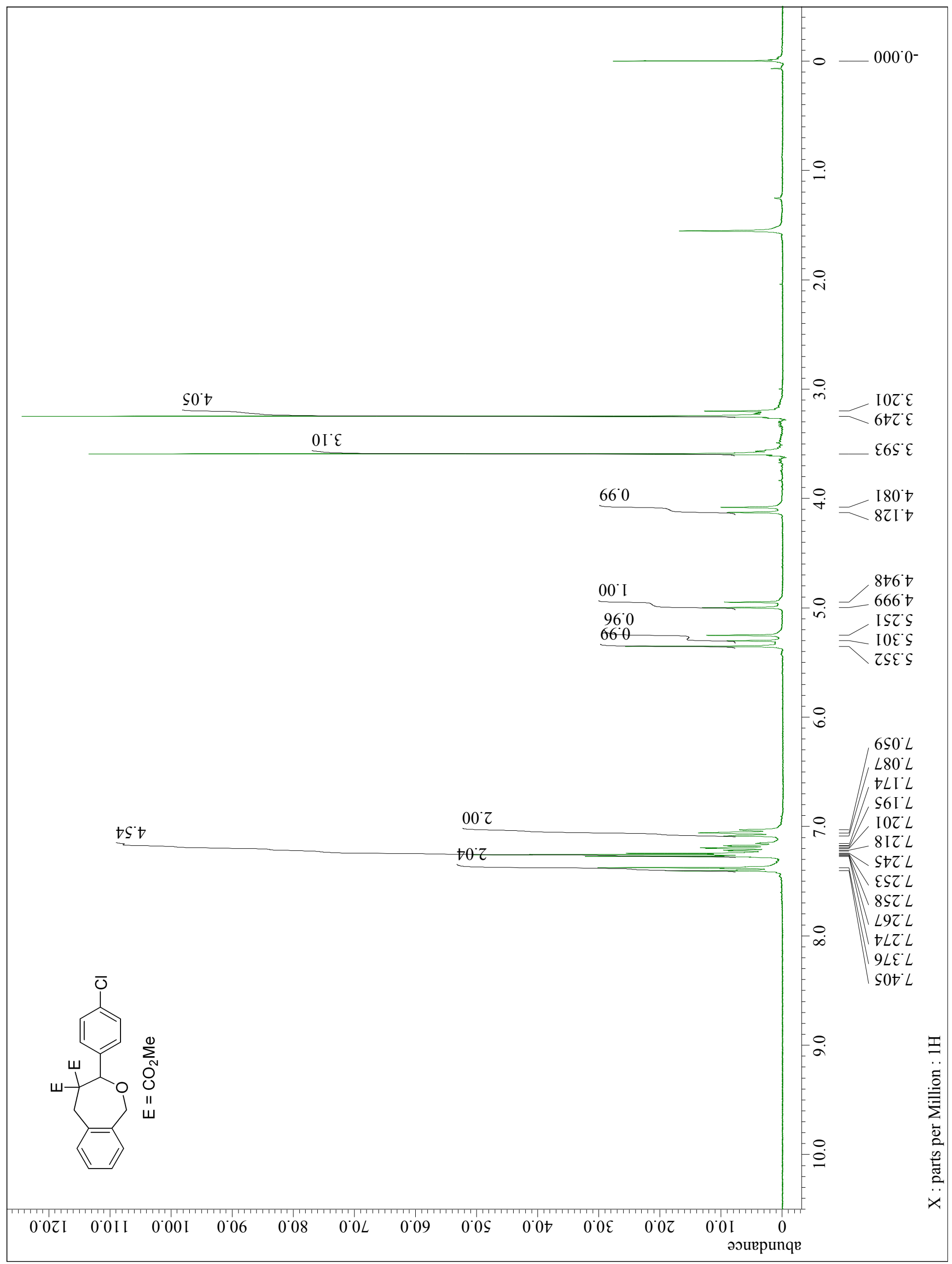


${ }^{13} \mathrm{C}$ NMR spectrum of $\mathbf{5 k}\left(\mathrm{CDCl}_{3}, 75 \mathrm{MHz}\right)$.

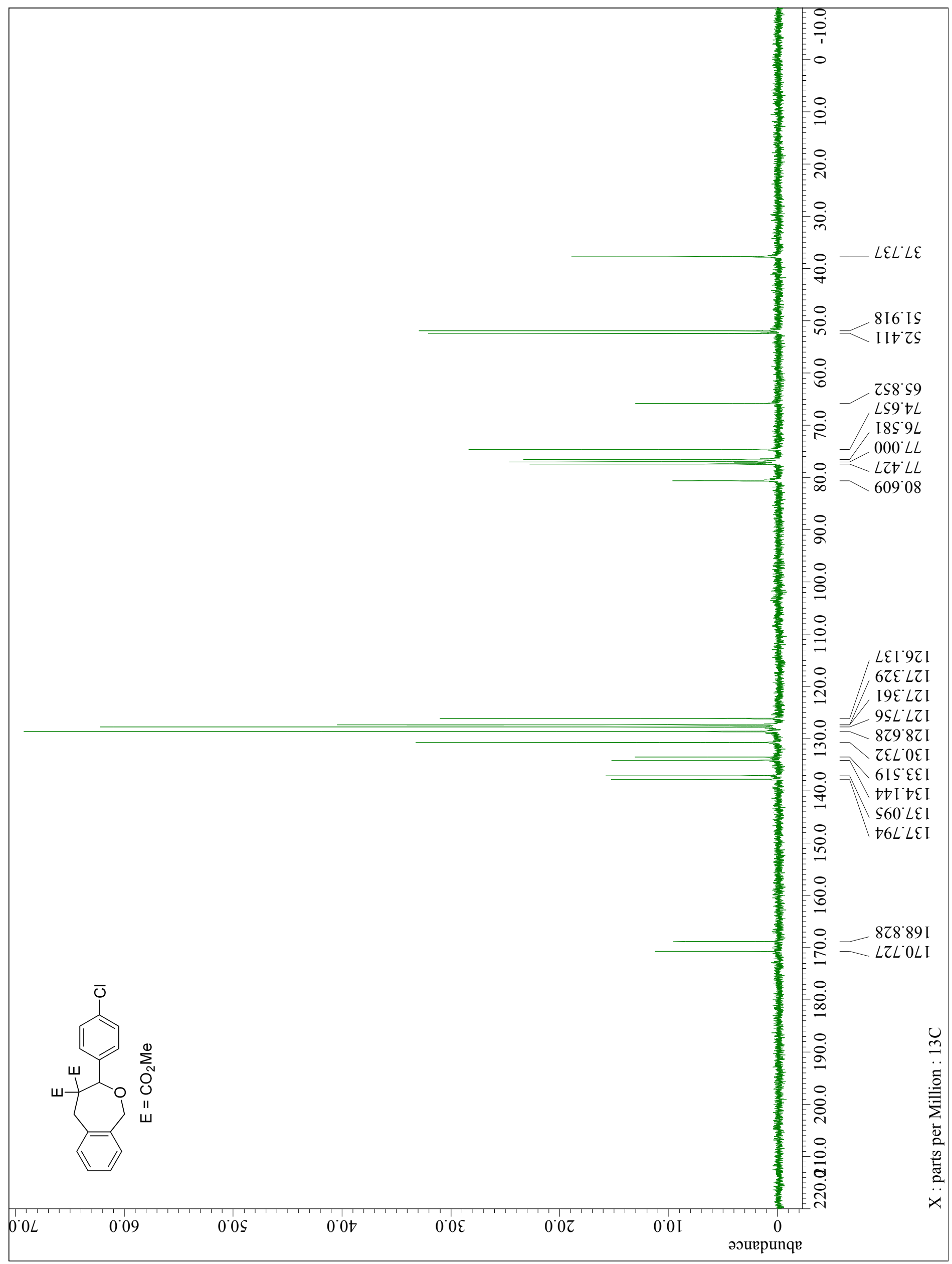


${ }^{1} \mathrm{H}$ NMR spectrum of $\mathbf{5 l}\left(\mathrm{CDCl}_{3}, 300 \mathrm{MHz}\right)$.

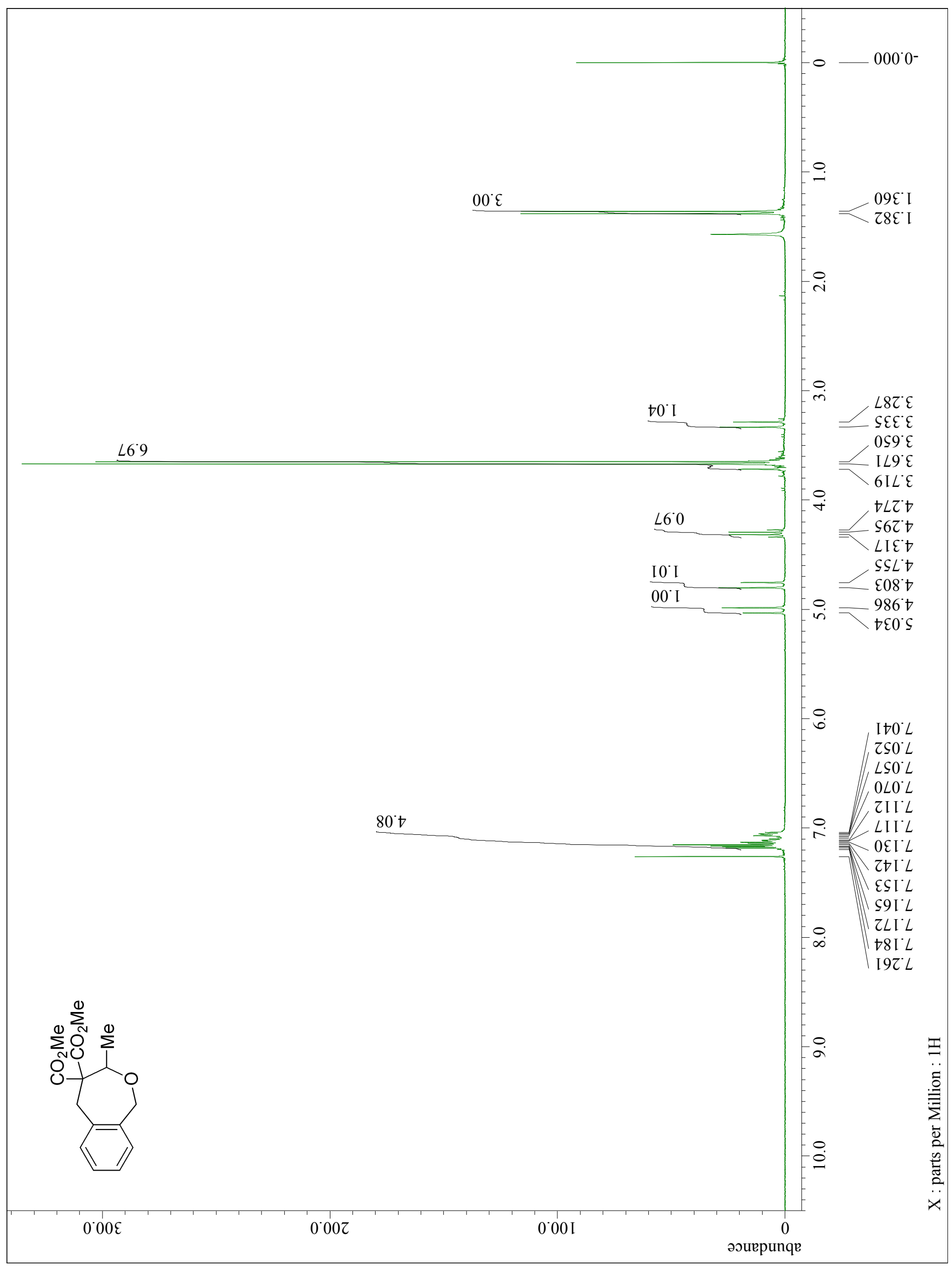


${ }^{13} \mathrm{C} \mathrm{NMR}$ spectrum of $\mathbf{5 l}\left(\mathrm{CDCl}_{3}, 75 \mathrm{MHz}\right)$.

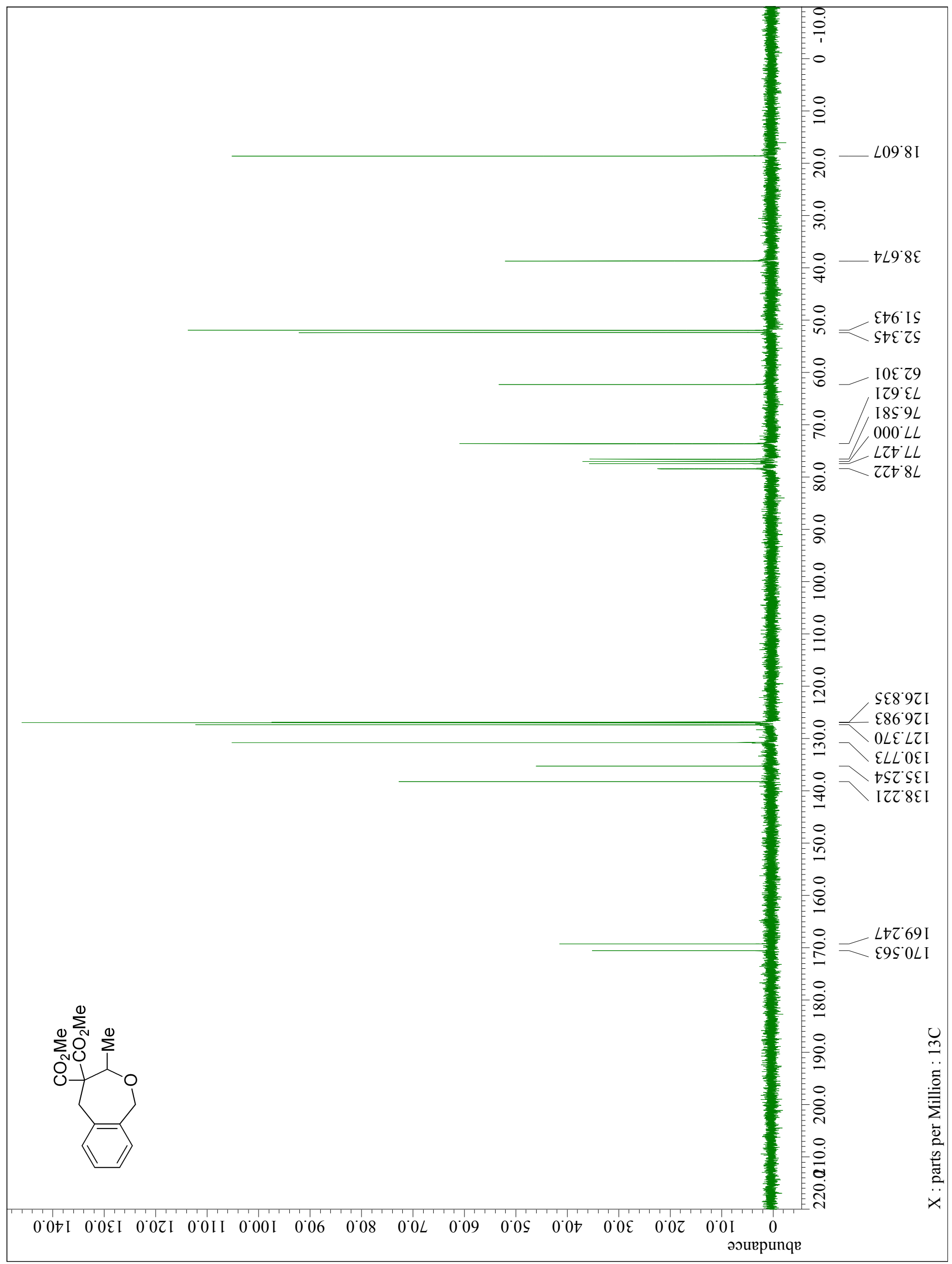

